\title{
Novel indication and optimization strategies for cardiac pacing
}

Citation for published version (APA):

Schreurs, R. (2021). Novel indication and optimization strategies for cardiac pacing. [Doctoral Thesis, Maastricht University]. Ridderprint. https://doi.org/10.26481/dis.20210910hs

Document status and date:

Published: 01/01/2021

DOI:

10.26481/dis.20210910hs

Document Version:

Publisher's PDF, also known as Version of record

\section{Please check the document version of this publication:}

- A submitted manuscript is the version of the article upon submission and before peer-review. There can be important differences between the submitted version and the official published version of record.

People interested in the research are advised to contact the author for the final version of the publication, or visit the DOI to the publisher's website.

- The final author version and the galley proof are versions of the publication after peer review.

- The final published version features the final layout of the paper including the volume, issue and page numbers.

Link to publication

\footnotetext{
General rights rights.

- You may freely distribute the URL identifying the publication in the public portal. please follow below link for the End User Agreement:

www.umlib.nl/taverne-license

Take down policy

If you believe that this document breaches copyright please contact us at:

repository@maastrichtuniversity.nl

providing details and we will investigate your claim.
}

Copyright and moral rights for the publications made accessible in the public portal are retained by the authors and/or other copyright owners and it is a condition of accessing publications that users recognise and abide by the legal requirements associated with these

- Users may download and print one copy of any publication from the public portal for the purpose of private study or research.

- You may not further distribute the material or use it for any profit-making activity or commercial gain

If the publication is distributed under the terms of Article $25 \mathrm{fa}$ of the Dutch Copyright Act, indicated by the "Taverne" license above, 


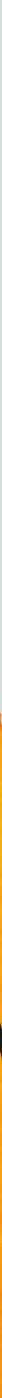

OPPIMIZATION STRATEGIES

AOR CARDIAC PACING
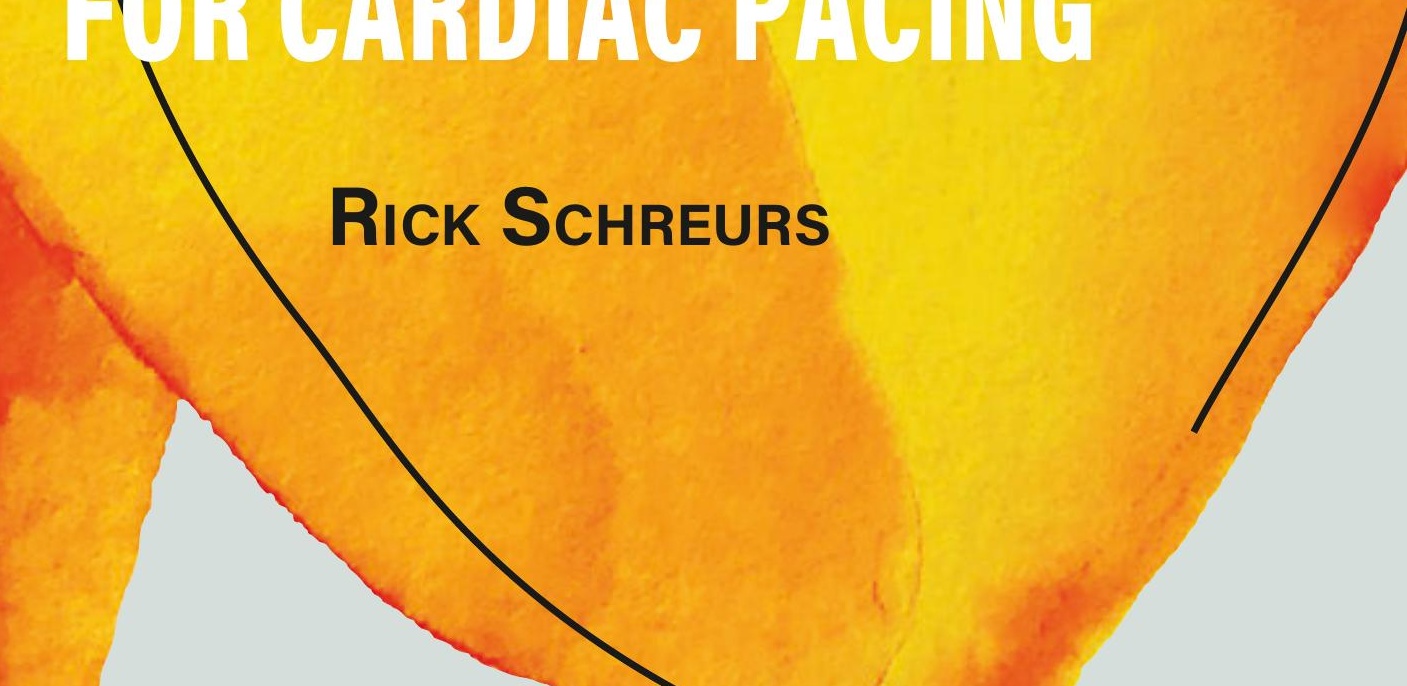



\section{NOVEL INDICATION AND OPTIMIZATION STRATEGIES FOR CARDIAC PACING}

RICK SCHREURS 


\section{NOVEL INDICATION AND OPTIMIZATION STRATEGIES FOR CARDIAC PACING}

Copyright () 2021 Henricus Hendrikus Hermanus Schreurs. All rights reserved. No part of this thesis may be reproduced, stored or transmitted in any way or by any means without the prior permission of the author, or when applicable, of the publishers of the scientific papers.

Cover design: Rosie Zopfi

Layout: Vera van Ommeren I persoonlijkproefschrift.nl

Printing: Ridderprint | www.ridderprint.nl

ISBN: 978-94-6416-703-0 


\title{
NOVEL INDICATION AND OPTIMIZATION STRATEGIES FOR CARDIAC PACING
}

\author{
PROEFSCHRIFT \\ ter verkrijging van de graad van doctor aan de Universiteit Maastricht, \\ op gezag van de Rector Magnificus, Prof. dr. Rianne M. Letschert \\ volgens het besluit van het College van Decanen, \\ in het openbaar te verdedigen \\ op vrijdag 10 september 2021 om 10.00u
}

door

Henricus Hendrikus Hermanus Schreurs

Geboren op 14 december 1985 te Venlo, Nederland 


\section{Promotores}

Prof. dr. F.W. Prinzen

Prof. dr. J.G. Maessen

\section{Beoordelingscommissie}

Prof. dr. U. Schotten (Chair)

Prof. dr. R. Lorusso

Prof. dr. H.P. Brunner-La Rocca

Prof. dr. A.J.J.C. Bogers (Erasmus MC)

Prof. dr. Frank Timmermans (UZ Gent)

Financial support by the Dutch Heart Association and Hart en vaat onderzoekfonds Limburg for the publication of this thesis is gratefully acknowledged 


\section{TABLE OF CONTENTS}

CHAPTER 1 General introduction 7

CHAPTER 2 Exploring the electrophysiological and hemodynamic 21 effects of cardiac resynchronization therapy. From bench to bedside and vice versa.

CHAPTER 3 Atrioventricular dromotropathy as substrate for successful pacing therapy in heart failure patients: a combined computational-experimental-clinical study

CHAPTER 4 Effective mechanical atrioventricular delay covers the impact of various pacing modes on cardiac pump function

CHAPTER 5 Pathophysiological interpretation of SonR sensor signals for resynchronization therapy of pacing delays in cardiac resynchronization therapy. A combined experimental-computational approach

CHAPTER 7 General discussion

Impact

Summary/Samenvatting

Dankwoord

About the author 




\section{INTRODUCTION}

The heart is the central organ of the cardiovascular system. Venous blood is collected in the right side of the heart and subsequently pumped to the lungs for oxygenation. The left side of the heart pumps the oxygenated blood to the systemic circulation. Each side of the heart consists of an atrium for collection of blood and a ventricle that ensures distribution to the pulmonary and systemic circulation. These chambers require coordinated activation and contraction in order to work optimally. The contraction is triggered by electrical stimuli in the heart (Figure 1). The first electrical stimulus derives from the sinus node in the upper part of the right atrium (RA). The electrical wave front activates the RA and left atrium (LA) via Bachman's Bundle and reaches the atrioventricular (AV) node via the internodal pathways where electrical propagation is slowed down compared to other cardiac tissue, causing a delay between atrial and ventricular activation. The ventricles are activated via the fast conduction His bundle and the left and right bundle branch towards the Purkinje fibers. From here the electrical impulses travel further through the slower conducting myocardial tissue in an endocardial-to-epicardial direction [1-3]. The result is a synchronous contraction of the right (RV) and left ventricle (LV), because all ventricular segments are activated in a short moment of time.

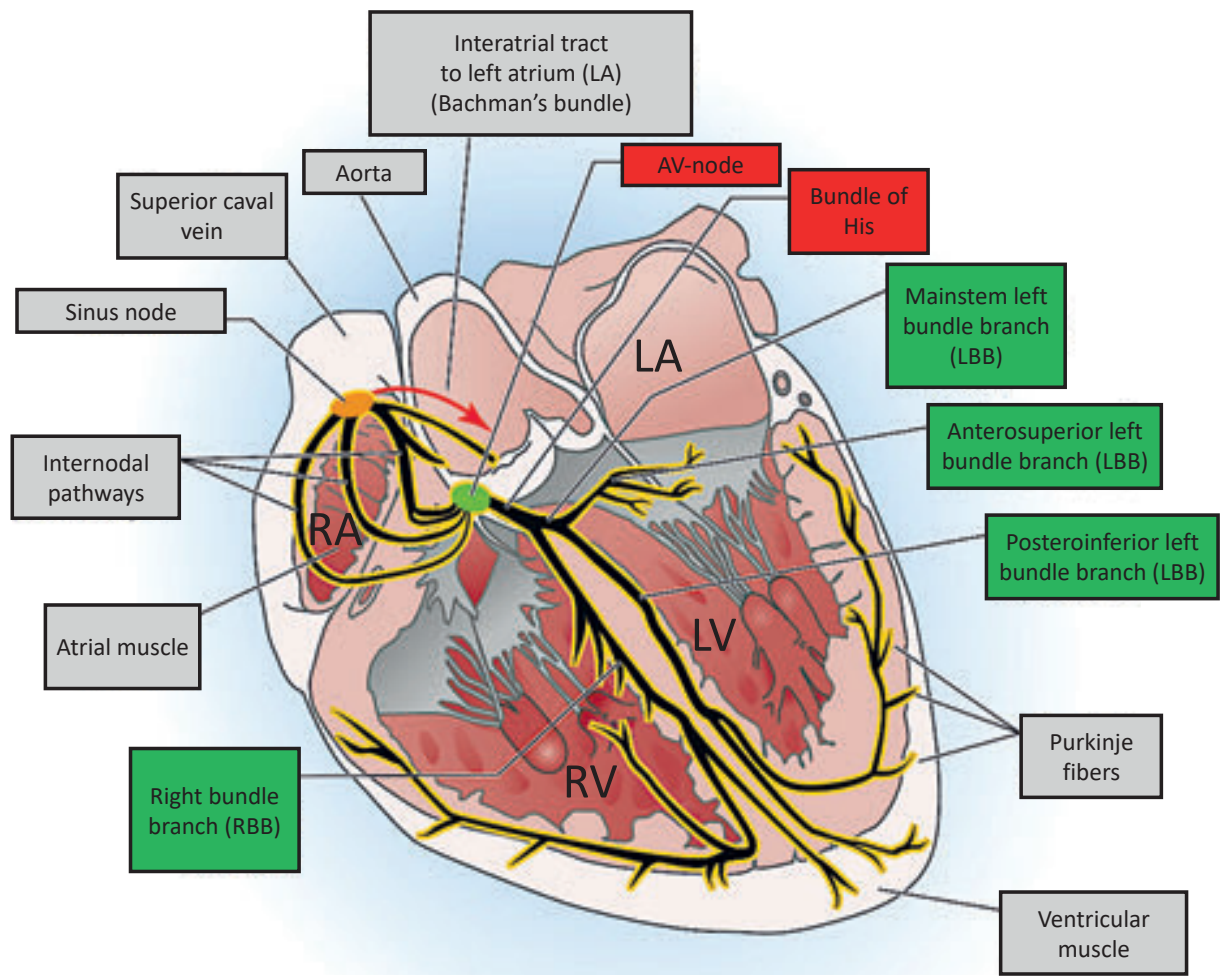

Figure 1. Conduction pathways through the heart. Total AV-block occurs at the level of the AV-node or Bundle of His (in red), while right (RBBB) and left bundle branch block (LBBB) are caused by damage to the RBB and LBB, respectively (in green). Adapted from [3]. 


\section{Conduction disorders}

Disturbances in the physiologic electrical conduction of the heart may lead to diminished cardiac function. Conduction disturbances can take place at various places in the heart. When electrical currents are blocked or delayed at the level of the AV-node an AV-block is present. There are three types of AV-block to be distinguished:

- First-degree AV-block: every P-wave is followed by a QRS complex with an interval $>200 \mathrm{~ms}$. In other words, every atrial contraction is followed by ventricular contraction, but the interval between the two is delayed and longer than $200 \mathrm{~ms}$.

- Second-degree AV-block: some P-waves are followed by a QRS complex while others are not. This blockage can be regular or at a random base.

- Third-degree AV-block: Also known as total AV-block; conduction between the atria and ventricles is completely blocked. The heart relies on an escape rhythm which is usually slower than the normal sinus rhythm.

AV-conduction delay leads to a mismatch between atrial and ventricular contraction and may cause a lower ventricular rate, which may alter ventricular filling and thereby induce heart failure.

Conduction disturbances can also occur at the level of the rapid conduction system of the ventricles, of which right (RBBB) and left bundle branch block (LBBB) are important examples. Because in these cases a large proportion of the ventricular myocardium is electrically activated through slow cell-to-cell conduction, RBBB and LBBB lead to prolonged electrical activation between the RV and LV (interventricular dyssynchrony) and within the affected ventricle (intraventricular delay). Because each cardiac myocyte requires the electrical impulse to start the contraction, the abnormal electrical activation causes disturbances in the coordination of mechanical contraction between and within ventricles, which may result in reduced cardiac pump function $[4,5]$.

\section{Heart failure}

Heart failure is a clinical syndrome characterized by a combination of signs and symptoms caused by structural and/or functional cardiac abnormalities, resulting in elevated intracardiac filling pressures and decreased cardiac output [6]. The prevalence of heart failure is $1-2 \%$ and predominantly affects older people. The etiology of heart failure is diverse with ischemic, valvular and hypertensive heart disease being most prevalent. Other causes include inflammation, toxic, immune and tachycardia, but also dyssynchrony and conduction disorders can lead to heart failure [6]. Often several causes interact in one patient making heart failure a challenging disease to treat. Different etiologies require different therapies, and although treatment has significantly improved over the last decades, prognosis of heart failure remains poor with high mortality (annual rate $7-17 \%$ ) and hospitalization rate (32-44\%) [7].

Besides trying to take away the underlying cause, treatment of heart failure primarily focusses on lifestyle changes and pharmacotherapy to reduce cardiac stress and filling 
pressures. Implantable cardioverter defibrillators (ICD) are indicated in patients with reduced LV ejection fraction or life-threatening arrhythmias to prevent sudden cardiac death. In therapy resistant end-stage heart failure left ventricular assist devices or heart transplantation should be considered [6].

\section{Cardiac resynchronization therapy}

Patients with electrical conduction disorders may also benefit from cardiac resynchronization therapy (CRT) using biventricular pacemakers [8-10]. CRT is a pacemaker therapy that aims to correct the dyssynchronous electrical activation and consequently improve cardiac pump function. CRT emerged during the 1990s and became FDA approved in 2001. Generally, CRT is performed through simultaneous (biventricular) pacing of both ventricles by one pacemaker lead in the RV apex and one lead in an epicardial vein on the posterolateral wall of the LV, accompanied by a RA lead to sense or pace atrial electrical activation [11]. CRT has proven to be more successful than pharmacological treatment, especially in patients with reduced LV ejection (<35\%) and LBBB with increased QRS duration (>130ms). It acutely improves cardiac output and LV systolic pressure [12] and at the long term leads to reverse remodeling (reduction of ventricular dimensions) and increased LV ejection fraction [13]. Patients benefit through decreased mortality and hospitalization rate and an increase in quality of life [14].

\section{Indications for CRT}

According to the guidelines of the European Society of Cardiologists, CRT is currently recommended for symptomatic heart failure patients with a QRS duration $\geq 130 \mathrm{~ms}$ and LBBB morphology on the electrocardiogram and with LV ejection fraction $\leq 35 \%$ despite optimal medical therapy $[6,8]$. In patients with non-LBBB morphology it should be considered when QRS duration $\geq 150 \mathrm{~ms}$ and may be considered in QRS 130-149ms. Furthermore, CRT is recommended over RV-only pacing in patients with heart failure with reduced ejection fraction who have an indication for ventricular pacing due to a high degree AV block. Patients with reduced ejection fraction who clinically worsen after initiating RVonly pacing may be considered for an upgrade to CRT [6].

The abovementioned indications of CRT focus on reducing interventricular dyssynchrony. Interesting, a few years before CRT merged, a few small studies indicated that also patients with heart failure and a prolonged PR-interval (first-degree AV-block) suggested significant functional and symptomatic improvement with restored AV coupling [15-17]. In this case $\mathrm{RV}$ pacing was used to normalize AV-delay and to restore AV coupling. With the rise of CRT, this possible application of pacemaker therapy lost attention, until a few years ago sub-analyses of clinical trials investigating the benefit of CRT revitalized the interest in this topic [18]. In sub-studies of the MADIT-CRT and COMPANION trial, only non-LBBB patients with prolonged PR-interval showed a significant clinical improvement to CRT, which was not present in non-LBBB patients with normal AV conduction $[19,20]$. A sub-study of the ReThinQ trial investigated the benefit of CRT in patients with QRS duration $<130 \mathrm{~ms}$, and 
found a significant increase in maximum oxygen uptake only patients with a prolonged PR-interval (>180ms) [21]. However, the acute hemodynamic improvements and underlying mechanisms of restoration of AV coupling in patients or animals with prolonged PR-interval have not been studies prospectively.

\section{Optimization of CRT}

Unfortunately, a vast number of patients does not show any significant response to CRT, although they completely fulfill the strict criteria $[22,23]$. The response to CRT depends on multiple factors like the underlying pathology and the mode of delivery. Optimization of CRT is multifactorial as well and can be divided into the following three parts [24]:

- Pre-implantation: better patient selection and optimizing imaging techniques.

- Implantation: optimal PM lead placement and focus on acute improvement.

- Post-implantation: program pacemaker settings for better timing of atrial and ventricular activation and improved follow-up.

This thesis focuses mainly on the optimization of pacemaker settings. All modern pacemakers possess the ability to individually program the delay between atrial and ventricular activation (AV-delay) and the delay between RV and LV pacing ( VV-delay, in case of CRT). Numerous studies have shown that optimizing the AV-delay improves ventricular filling, while optimizing the interventricular delay significantly reduces dyssynchrony and improves ventricular activation patterns $[25,26]$.

Several studies using single, in rest AV optimization were not able to show a longterm benefit of CRT as compared to using a default setting [27-29]. However, acute hemodynamic studies have shown benefit of CRT optimization and that the optimal AVdelay varies from one patient to another and may change between rest and exercise [3033]. Most dynamic, automated algorithms for AV optimization are based on electrical signals like electrograms or electrocardiography. The Adaptive CRT algorithm provides LV-only pacing timed with native RV activation, optimizing AV and VV-delay on the basis of periodic automatic evaluation of intrinsic conduction intervals [28, 34, 35]. The SyncAV algorithm provides a concept of BiV pacing by periodically measuring intrinsic conduction and dynamically adjusting AV-delays $50 \mathrm{~ms}$ shorter than the intrinsic AV-interval, allowing paced BiV wavefronts to fuse with intrinsic conduction (triple wavefront fusion) [36, 37]. The QuickOpt optimization algorithm examines the conduction properties of the heart during various spontaneous and paced rhythms and calculates the optimal AV and VV delays that achieve the best electromechanical resynchronization of the LV [38, 39].

The SonR signal, or peak endocardial acceleration, is the only algorithm that is based on a mechanical sensor. The signal, which is derived from intracardiac accelerometers, is related to the amplitude of the first heart sound and shows a strong correlation with cardiac contractility [40]. An automated algorithm based on the SonR signal has been proven to be non-inferior to echocardiography-based optimization in terms of clinical outcome in 
patients with CRT $[41,42]$. To our believe, better understanding of this signal might lead to improvements in the optimization algorithm.

\section{Animal studies}

Animals have long been used to study dyssynchrony and resynchronization using pacemakers. The LBBB has been described in monkeys and pigs in a manner that is comparable to humans. In other animals like ox and sheep, the bundles are significantly thicker and their branches extend much more towards the epicardium compared to the human situation. In rabbit hearts the left 'bundle' is more a group of fine sheets covering the subendocardial tissue [43]. To study CRT in animals a model with sufficient amount of interventricular dyssynchrony is preferable. In pigs RV pacing (to mimic LBBB) or LBBB increases QRS duration by only 50\% [44] and the increase is even less in goats. The most optimal species is the dog, in which a doubling of QRS duration can be seen during LBBB which is comparable to human. Percutaneous radiofrequency ablation of the LBBB in canine hearts results in a model perfectly suited to study the effects of CRT [45-48].

\section{Computational modeling of the heart}

Computer models provide a description of situations through simulations, based on assumed relations and simulation conditions. This provides a level of control that is not always physically or ethically possible in animals or clinical studies, which raises the opportunity to study new insights in the context of CRT [49]. In the current thesis CircAdapt was used to study the influence of various pacemaker settings on hemodynamic outcome. CircAdapt is a model that consists of various modules representing not only the atria, ventricles and myocardial tissue, but also pericardium, heart valves, large blood vessels and systemic and pulmonary circulation [50,51]. The CircAdapt model is able to simulate cardiac pacing and to describe the physiology and physics of AV-coupling and the mechanical and electrical interaction of the ventricles. The model has been extensively used to generate additional insights of existing clinical and animal experimental data or to form new hypotheses in the context of cardiac pacing [52-55].

\section{General aims and outline of the thesis}

The research presented in this thesis has two main aims. The first aim is to examine if CRT improves cardiac function in patients with prolonged PR-interval and whether first-degree AV-block (AV dromotropathy) might therefore be a new indication for CRT. The second aim is to explore new ways of optimizing pacemaker settings in CRT and relate them to hemodynamic outcome.

After this general introduction, chapter 2 reviews the electrophysiological and hemodynamic changes that occur during CRT in both patient and animal studies. It elaborates on electromechanics of dyssynchrony and how CRT reverses this. Furthermore, it focusses on the acute hemodynamic improvements that can be seen due to CRT and on the long-term reverse remodeling of the heart. It reviews the influence of various pacing modalities and 
myocardial scarring on the effect of CRT. And finally, the optimization of AV-and VV-delays in patients and animals are discussed.

The first-degree AV-block as a new indication for CRT has been studied in chapter 3. The chapter is a combination of clinical, computational and animal experiments. It describes the acute hemodynamic improvements in patients with reduced LV ejection fraction and prolonged PR-intervals. The same has been done in a porcine model of first-degree AVblock. Additionally, these experiments were replicated using the CircAdapt computer model. Overall, special attention has been paid to the improvements in cardiac function and the optimization of cardiac filling pressures using invasive measurements.

In chapter $\mathbf{4}$ a porcine model of total AV-block was used to study whether atrial sensing or atrial pacing was superior to the other in cardiac pacing and how the variance in interatrial delay (IAD) led to differences in the optimal AV-delay. Additionally, the interventricular dyssynchrony was varied by changing ventricular pacing sites in order to study its influence on the right and left effective, mechanical AV-delay. Main goal was to find a new parameter to better predict the optimal AV-delay irrespective of IAD or ventricular pacing.

In chapter 5 a canine LBBB model was used to gain more information on the pathophysiology of the SonR signal and the aim was to find new insights in how to further improve the algorithm to optimize the AV-delay

Finally, a combined experimental-computational approach was chosen in chapter $\mathbf{6}$ to examine the response of the LV and RV to various pacing delays in CRT. Data from experiments in canine LBBB hearts were simulated using CircAdapt to study the differences between $L V$ and $R V$ hemodynamic response and linked to find the optimal overall settings.

To conclude, chapter $\mathbf{7}$ integrates the major finding of the above-mentioned studies and discusses them in a broader scientific and clinical perspective. 


\section{REFERENCES}

1 Durrer D, van Dam RT, Freud GE, Janse MJ, Meijler FL, Arzbaecher RC. Total excitation of the isolated human heart. Circulation. 1970;41(6):899-912.

2 Mulpuru SK, Madhavan M, McLeod CJ, Cha YM, Friedman PA. Cardiac Pacemakers: Function, Troubleshooting, and Management: Part 1 of a 2-Part Series. J Am Coll Cardiol. 2017;69(2):189210.

3 Lederer W. Cardiac Electrophysiology and the Electrocardiogram. In: Boron WF, Boulpaep EL, editors. Medical physiology 2017.

4 Vernooy K, Verbeek XA, Peschar M, Crijns HJ, Arts T, Cornelussen RN, et al. Left bundle branch block induces ventricular remodelling and functional septal hypoperfusion. Eur Heart J. 2005;26(1):91-8.

5 Cheng A, Helm RH, Abraham TP. Pathophysiological mechanisms underlying ventricular dyssynchrony. Europace. 2009;11 Suppl 5:v10-4.

6 Ponikowski P, Voors AA, Anker SD, Bueno H, Cleland JGF, Coats AJS, et al. 2016 ESC Guidelines for the diagnosis and treatment of acute and chronic heart failure: The Task Force for the diagnosis and treatment of acute and chronic heart failure of the European Society of Cardiology (ESC)Developed with the special contribution of the Heart Failure Association (HFA) of the ESC. Eur Heart J. 2016;37(27):2129-200.

7 Maggioni AP, Dahlstrom U, Filippatos G, Chioncel O, Crespo Leiro M, Drozdz J, et al. EURObservational Research Programme: regional differences and 1-year follow-up results of the Heart Failure Pilot Survey (ESC-HF Pilot). Eur J Heart Fail. 2013;15(7):808-17.

8 Brignole M, Auricchio A, Baron-Esquivias G, Bordachar P, Boriani G, Breithardt OA, et al. 2013 ESC guidelines on cardiac pacing and cardiac resynchronization therapy: the task force on cardiac pacing and resynchronization therapy of the European Society of Cardiology (ESC). Developed in collaboration with the European Heart Rhythm Association (EHRA). Europace. 2013;15(8):1070-118.

9 Cleland JG, Daubert JC, Erdmann E, Freemantle N, Gras D, Kappenberger L, et al. The effect of cardiac resynchronization on morbidity and mortality in heart failure. $\mathrm{N}$ Engl J Med. 2005;352:1539-49.

10 Moss AJ, Hall WJ, Cannom DS, Klein H, Brown MW, Daubert JP, et al. Cardiac-resynchronization therapy for the prevention of heart-failure events. N Engl J Med. 2009;361(14):1329-38.

11 Abraham WT, Fisher WG, Smith AL, Delurgio DB, Leon AR, Loh E, et al. Cardiac resynchronization in chronic heart failure. N Engl J Med. 2002;346(24):1845-53.

12 de Roest GJ, Allaart CP, Kleijn SA, Delnoy PP, Wu L, Hendriks ML, et al. Prediction of long-term outcome of cardiac resynchronization therapy by acute pressure-volume loop measurements. Eur J Heart Fail. 2013;15(3):299-307.

13 Ghio S, Freemantle N, Scelsi L, Serio A, Magrini G, Pasotti M, et al. Long-term left ventricular reverse remodelling with cardiac resynchronization therapy: results from the CARE-HF trial. Eur $J$ Heart Fail. 2009;11(5):480-8.

14 Colquitt JL, Mendes D, Clegg AJ, Harris P, Cooper K, Picot J, et al. Implantable cardioverter defibrillators for the treatment of arrhythmias and cardiac resynchronisation therapy for the treatment of heart failure: systematic review and economic evaluation. Health Technol Assess. 2014;18(56):1-560.

15 Brecker SJ, Xiao HB, Sparrow J, Gibson DG. Effects of dual-chamber pacing with short atrioventricular delay in dilated cardiomyopathy. Lancet. 1992;340(8831):1308-12.

16 Hochleitner M, Hortnagl H, Hortnagl H, Fridrich L, Gschnitzer F. Long-term efficacy of physiologic dual-chamber pacing in the treatment of end-stage idiopathic dilated cardiomyopathy. Am $\mathrm{J}$ Cardiol. 1992;70(15):1320-5.

17 Sack S, Franz R, Dagres N, Oldenburg O, Herrmann J, Golles A, et al. Can right-sided atrioventricular sequential pacing provide benefit for selected patients with severe congestive heart failure? Am J Cardiol. 1999;83(5B):124D-9D. 
18 Salden F, Kutyifa V, Stockburger M, Prinzen FW, Vernooy K. Atrioventricular dromotropathy: evidence for a distinctive entity in heart failure with prolonged PR interval? Europace. 2018;20(7):1067-77.

19 Kutyifa V, Stockburger M, Daubert JP, Holmqvist F, Olshansky B, Schuger C, et al. PR interval identifies clinical response in patients with non-left bundle branch block: a Multicenter Automatic Defibrillator Implantation Trial-Cardiac Resynchronization Therapy substudy. Circ Arrhythm Electrophysiol. 2014;7(4):645-51.

20 Lin J, Buhr KA, Kipp R. Effect of PR Interval on Outcomes Following Cardiac Resynchronization Therapy: A Secondary Analysis of the COMPANION Trial. J Cardiovasc Electrophysiol. 2017;28(2):185-91.

21 Joshi NP, Stopper MM, Li J, Beshai JF, Pavri BB. Impact of baseline PR interval on cardiac resynchronization therapy outcomes in patients with narrow QRS complexes: an analysis of the ReThinQ Trial. J Interv Card Electrophysiol. 2015;43(2):145-9.

22 Naqvi SY, Jawaid A, Goldenberg I, Kutyifa V. Non-response to Cardiac Resynchronization Therapy. Curr Heart Fail Rep. 2018;15(5):315-21.

23 Zhang Q, Zhou Y, Yu CM. Incidence, definition, diagnosis, and management of the cardiac resynchronization therapy nonresponder. Curr Opin Cardiol. 2015;30(1):40-9.

24 Vernooy K, van Deursen CJ, Strik M, Prinzen FW. Strategies to improve cardiac resynchronization therapy. Nat Rev Cardiol. 2014;11(8):481-93.

25 Auger D, Hoke U, Thijssen J, Abate E, Yiu KH, Ewe SH, et al. Effect of cardiac resynchronization therapy on the sequence of mechanical activation assessed by two-dimensional radial strain imaging. Am J Cardiol. 2014;113(6):982-7.

26 Strik M, van Middendorp LB, Houthuizen P, Ploux S, van Hunnik A, Kuiper M, et al. Interplay of electrical wavefronts as determinant of the response to cardiac resynchronization therapy in dyssynchronous canine hearts. Circ Arrhythm Electrophysiol. 2013;6(5):924-31.

27 Zhang Y, Xing Q, Zhang JH, Jiang WF, Qin M, Liu X. Long-Term Effect of Different Optimizing Methods for Cardiac Resynchronization Therapy in Patients with Heart Failure: A Randomized and Controlled Pilot Study. Cardiology. 2019;142(3):158-66.

28 Martin DO, Lemke B, Birnie D, Krum H, Lee KL, Aonuma K, et al. Investigation of a novel algorithm for synchronized left-ventricular pacing and ambulatory optimization of cardiac resynchronization therapy: results of the adaptive CRT trial. Heart Rhythm. 2012;9(11):1807-14.

29 Abraham WT, Gras D, Yu CM, Calo L, Islam N, N K. Results from the FREEDOM trial: assess the safety and efficacy of frequent optimization of cardiac resynchronization therapy. Presented at Heart Rhythm Society 31st Annual Scientific Sessions Denver. 2010.

30 Auricchio A, Stellbrink C, Sack S, Block M, Vogt J, Bakker P, et al. The Pacing Therapies for Congestive Heart Failure (PATH-CHF) study: rationale, design, and endpoints of a prospective randomized multicenter study. Am J Cardiol. 1999;83(5B):130D-5D.

31 Bogaard MD, Kirkels JH, Hauer RN, Loh P, Doevendans PA, Meine M. Should we optimize cardiac resynchronization therapy during exercise? J Cardiovasc Electrophysiol. 2010;21(11):1307-16.

32 Zweerink A, Salden OAE, van Everdingen WM, de Roest GJ, van de Ven PM, Cramer MJ, et al. Hemodynamic Optimization in Cardiac Resynchronization Therapy: Should We Aim for dP/ dtmax or Stroke Work? JACC Clin Electrophysiol. 2019;5(9):1013-25.

33 Whinnett ZI, Davies JE, Willson K, Manisty CH, Chow AW, Foale RA, et al. Haemodynamic effects of changes in atrioventricular and interventricular delay in cardiac resynchronisation therapy show a consistent pattern: analysis of shape, magnitude and relative importance of atrioventricular and interventricular delay. Heart. 2006;92(11):1628-34.

34 Krum H, Lemke B, Birnie D, Lee KL, Aonuma K, Starling RC, et al. A novel algorithm for individualized cardiac resynchronization therapy: rationale and design of the adaptive cardiac resynchronization therapy trial. Am Heart J. 2012;163(5):747-52 e1.

35 Burns KV, Gage RM, Curtin AE, Gorcsan J, 3rd, Bank AJ. Left ventricular-only pacing in heart failure patients with normal atrioventricular conduction improves global function and left ventricular regional mechanics compared with biventricular pacing: an adaptive cardiac resynchronization therapy sub-study. Eur J Heart Fail. 2017;19(10):1335-43. 
36 Thibault B, Ritter P, Bode K, Calo L, Mondesert B, Mangual JO, et al. Dynamic programming of atrioventricular delay improves electrical synchrony in a multicenter cardiac resynchronization therapy study. Heart Rhythm. 2019;16(7):1047-56.

37 Varma N, O'Donnell D, Bassiouny M, Ritter P, Pappone C, Mangual J, et al. Programming Cardiac Resynchronization Therapy for Electrical Synchrony: Reaching Beyond Left Bundle Branch Block and Left Ventricular Activation Delay. J Am Heart Assoc. 2018;7(3).

38 Abraham WT, Gras D, Yu CM, Guzzo L, Gupta MS, Committee FS. Rationale and design of a randomized clinical trial to assess the safety and efficacy of frequent optimization of cardiac resynchronization therapy: the Frequent Optimization Study Using the QuickOpt Method (FREEDOM) trial. Am Heart J. 2010;159(6):944-8 e1.

39 Porciani MC, Rao CM, Mochi M, Cappelli F, Bongiorno G, Perini AP, et al. A real-time threedimensional echocardiographic validation of an intracardiac electrogram-based method for optimizing cardiac resynchronization therapy. Pacing Clin Electrophysiol. 2008;31(1):56-63.

40 Rickards AF, Bombardini T, Corbucci G, Plicchi G. An implantable intracardiac accelerometer for monitoring myocardial contractility. The Multicenter PEA Study Group. Pacing Clin Electrophysiol. 1996;19(12 Pt 1):2066-71.

41 Brugada J, Delnoy PP, Brachmann J, Reynolds D, Padeletti L, Noelker G, et al. Contractility sensor-guided optimization of cardiac resynchronization therapy: results from the RESPONDCRT trial. Eur Heart J. 2016.

42 Ritter P, Delnoy PP, Padeletti L, Lunati M, Naegele H, Borri-Brunetto A, et al. A randomized pilot study of optimization of cardiac resynchronization therapy in sinus rhythm patients using a peak endocardial acceleration sensor vs. standard methods. Europace. 2012;14(9):1324-33.

43 Strik M, van Middendorp LB, Vernooy K. Animal models of dyssynchrony. J Cardiovasc Transl Res. 2012;5(2):135-45.

44 Marrouche NF, Pavia SV, Zhuang S, Kim YJ, Tabata T, Wallick D, et al. Nonexcitatory stimulus delivery improves left ventricular function in hearts with left bundle branch block. J Cardiovasc Electrophysiol. 2002;13(7):691-5.

45 Liu L, Tockman B, Girouard S, Pastore J, Walcott G, KenKnight B, et al. Left ventricular resynchronization therapy in a canine model of left bundle branch block. Am J Physiol Heart Circ Physiol. 2002;282(6):H2238-44.

46 Verbeek XA, Vernooy K, Peschar M, Cornelussen RN, Prinzen FW. Intra-ventricular resynchronization for optimal left ventricular function during pacing in experimental left bundle branch block. J Am Coll Cardiol. 2003;42(3):558-67.

47 Verbeek XA, Vernooy K, Peschar M, Van Der Nagel T, Van Hunnik A, Prinzen FW. Quantification of interventricular asynchrony during LBBB and ventricular pacing. Am J Physiol Heart Circ Physiol. 2002;283(4):H1370-8.

48 Vernooy K, Cornelussen RN, Verbeek XA, Vanagt WY, van Hunnik A, Kuiper M, et al. Cardiac resynchronization therapy cures dyssynchronopathy in canine left bundle-branch block hearts. Eur Heart J. 2007;28(17):2148-55.

49 Lee AWC, Costa CM, Strocchi M, Rinaldi CA, Niederer SA. Computational Modeling for Cardiac Resynchronization Therapy. J Cardiovasc TransI Res. 2018;11(2):92-108.

50 Arts T, Delhaas T, Bovendeerd P, Verbeek X, Prinzen FW. Adaptation to mechanical load determines shape and properties of heart and circulation: the CircAdapt model. Am J Physiol Heart Circ Physiol. 2005;288(4):H1943-54.

51 Lumens J, Delhaas T, Kirn B, Arts T. Three-wall segment (TriSeg) model describing mechanics and hemodynamics of ventricular interaction. Ann Biomed Eng. 2009;37(11):2234-55.

52 Huntjens PR, Ploux S, Strik M, Walmsley J, Ritter P, Haissaguerre M, et al. Electrical Substrates Driving Response to Cardiac Resynchronization Therapy: A Combined Clinical-Computational Evaluation. Circ Arrhythm Electrophysiol. 2018;11(4):e005647.

53 Jones S, Lumens J, Sohaib SMA, Finegold JA, Kanagaratnam P, Tanner M, et al. Cardiac resynchronization therapy: mechanisms of action and scope for further improvement in cardiac function. Europace. 2017;19(7):1178-86. 
54 Ploux S, Eschalier R, Whinnett ZI, Lumens J, Derval N, Sacher F, et al. Electrical dyssynchrony induced by biventricular pacing: Implications for patient selection and therapy improvement. Heart Rhythm. 2015;12(4):782-91.

55 Walmsley J, Arts T, Derval N, Bordachar P, Cochet H, Ploux S, et al. Fast Simulation of Mechanical Heterogeneity in the Electrically Asynchronous Heart Using the MultiPatch Module. PLoS Comput Biol. 2015;11(7). 




\section{SYNOPSIS}

Cardiac resynchronization therapy (CRT) is an important therapy for heart failure patients with prolonged QRS duration. In patients with left bundle branch block the altered left ventricular electrical activation results in dyssynchronous, inefficient contraction of the left ventricle. CRT aims to reverse these changes and to improve cardiac function. This review explores the electrophysiological and hemodynamic changes that occur during CRT in both patient and animal studies. It also addresses how novel techniques such as multipoint and endocardial pacing can further improve the electromechanical response. 


\section{INTRODUCTION}

Patients with heart failure combined with left bundle branch block (LBBB) have broad QRS complexes and an impaired cardiac function. In these patients it is unclear whether heart failure leads to LBBB or vice versa. Animal models however, have shown that induction of LBBB reduces cardiac contractility [1]. Similarly, inadvertent induction of LBBB during Transcatheter Aortic Valve Replacement worsens outcome [2]. In LBBB cardiac function is impaired because the left (LV) and right ventricle (RV) and various regions within the LV are not activated simultaneously, leading to dyssynchronous contraction and reduced cardiac pump function [3]. Animal studies have shown that isolated LBBB causes reduction in ejection time, slower rates of rise and fall of the LV pressure and prolonged duration of isovolumic contraction and relaxation [4].

Cardiac resynchronization therapy (CRT) aims to restore synchronous contraction of the heart by either LV free wall or biventricular (BiV) pacing. The first studies started during the late 90s to evaluate the effect of CRT [5-7]. According to the current guidelines a CRT device is indicated for patients with heart failure and prolonged QRS duration, preferably with LBBB morphology on the electrocardiogram (ECG) [8]. CRT reduces mortality and improves quality of life [9], but additional research is needed to optimize this therapy. To this purpose investigations have also been performed in animal models of dyssynchrony.

Animals have long been used to study hemodynamics and electrophysiology in heart failure. As reviewed by Strik et al [10]. the canine appears to be the best species to study ventricular conduction abnormalities that occur during pacing and LBBB, because the anatomical structures of the bundle branches are comparable to those in human. Percutaneous radiofrequency ablation of the LBBB results in a model perfectly suited to study the effects of CRT $[1,4,11,12]$. This model enables the investigation of the role of LBBB and CRT in the absence of heart failure, but if needed, also in combination with heart failure (created by chronic tachypacing) or myocardial infarction [10]. The aim of this article is to review the electrophysiological and hemodynamic effects of CRT in animal models and patients with LBBB. 


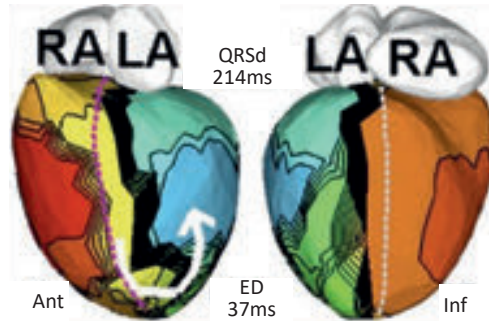

LV pacing
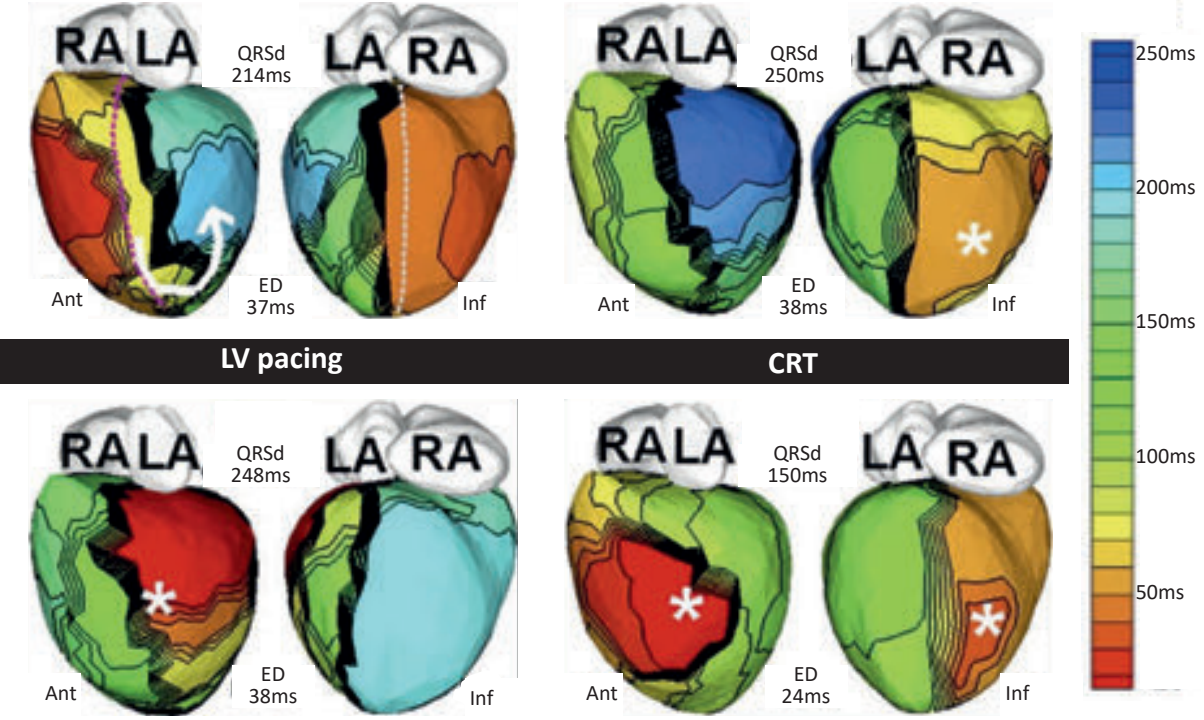

CRT

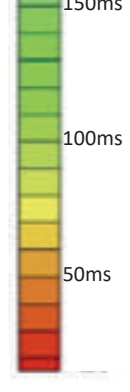

Figure 1. Electrocardiographic images of an LBBB patient recorded during intrinsic conduction, RV pacing, LV pacing and CRT. Each pair of images shows the anterior (Ant) and inferior (Inf) views. The black lines depict isochronal lines. Crowded isochronal lines indicate slow conduction. Thick black lines indicate conduction block. An asterisk indicates the pacing site. The dotted lines in the images of intrinsic conduction show the septal aspect of the epicardium. The QRS duration (QRSd) and the electrical dyssynchrony index (ED; calculated as the standard deviation of activation times at 500 sites in the LV) are indicated in the figure for each pacing modality. LA: left atrium, RA: right atrium. Reproduced with permission from the Heart Rhythm Society [13].

\section{Electro-mechanics of dyssynchrony}

In LBBB the normal activation pattern is disturbed because the LV is no longer activated via the left bundle branch and Purkinje fibers. Instead, the electrical activation spreads from the normally activated RV through the septum towards the LV. Since activation moving from myocyte to myocyte is much slower, the LV free wall - which is the site most remote from the RV - is activated latest. Several clinical $[14,15]$ and pre-clinical [16] invasive electrocardiac mapping studies have shown that the activation in LBBB hearts follows a specific pattern. LV depolarization moves from the septum in a circumferential and longitudinal direction. However, because conduction often appears slow at the RV-LV junctions an important contribution of activation comes from the wavefront passing over the apex towards the LV lateral wall (referred to as U-shaped activation pattern). Another characteristic feature is the slow transseptal conduction in LBBB, possibly caused by the vertical orientation of the laminar sheets of myocytes in the septum [17, 18]. Epicardial activation maps generated with non-invasive electrocardiographic imaging show comparable electrical patterns, as illustrated by the white arrow in the upper left panel of figure 1 [13]. The prolonged activation of the LV results in an increased total activation time 
(TAT) of both ventricles, which is characterized by a widened QRS complex on the surface ECG [14]. These findings are similar in dogs [10].

The left panel of figure 2 describes how the dyssynchronous electrical activation of the LV causes the early-activated septum to contract against a reduced load, which leads to pre-stretch of the LV lateral wall (i.e. more positive strain; middle panel, solid line) [14]. This pre-stretch increases contractile force of the LV free wall, which on its turn paradoxically stretches the septum later in systole (positive strain of the septum, striped line). Both types of systolic stretching can be considered wasted work, which is the ratio of negative and positive work (red and black lines respectively; bottom panels) [3, 19].

\section{LBBB}

Acute CRT
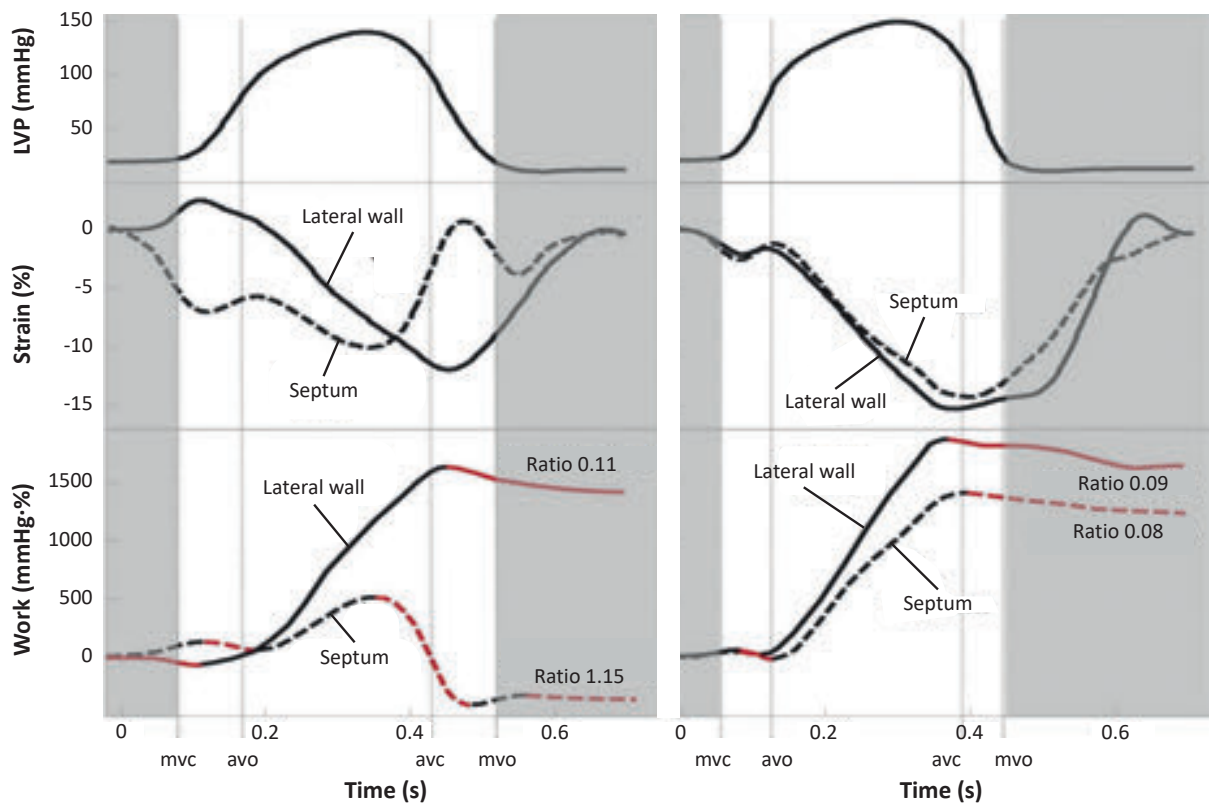

Figure 2. Estimated LV pressure (top), strain (middle), and calculation of wasted work for the septum and lateral wall (bottom) in a representative patient with left bundle branch block (LBBB; left) and after treatment with cardiac resynchronization therapy (CRT; right). Vertical lines indicate valvular events defined by echocardiography. Negative and positive work during systole is marked as red and black, respectively. Wasted work ratios were calculated using cumulated work between the closure and subsequent opening of the mitral valve (MVC and MVO, respectively). AVO: aortic valve opening, AVC: aortic valve closure. Reproduced with permission from the American Physiological Society [19].

In canine hearts, the maximum rates of rise and fall of the LV pressure ( $d P / d_{\max }$ and $\mathrm{dP}$ / $\left.\mathrm{dt}_{\text {min }}\right)$ decrease immediately upon creating LBBB and this decrease is still present after 8 weeks. Echocardiographic follow-up shows an increase of end-diastolic and end-systolic volume and decrease of ejection fraction with longer lasting LBBB [3]. These findings 
replicate the low ejection fraction, increased LV wall stress and end-systolic volume, and impaired myocardial relaxation as seen in LBBB patients [20].

\section{Electrophysiological effects of CRT}

The basic idea behind CRT is to resynchronize the late and more slowly activated LV by individually pacing both ventricles. Figure $\mathbf{1}$ shows that RV pacing alone results in an activation pattern that resembles the intrinsic activation pattern of LBBB and leads to a QRS duration of $250 \mathrm{~ms}$ in this example. At a short AV-delay LV pacing increases QRS duration, but with a completely reversed activation pattern compared to LBBB and RV pacing. With BiV pacing the left and right activation wavefronts fuse in the LV, which accelerates its activation, and consequently result in a shorter QRS duration of $150 \mathrm{~ms}$.

In dogs simultaneous RV and LV pacing shortens TAT and QRS duration compared to the LBBB situation and immediately improves hemodynamics [10]. However, note that BiV pacing does not necessarily reduce QRS duration [6, 7] or TAT [15], which can be explained by prolonged activation of the RV that is not intrinsically activated anymore (i.e. slower cell-to-cell conduction instead of activation through the Purkinje network; compare intrinsic conduction and RV pacing in figure 1). Moreover, in dogs it has been shown that hemodynamic improvement can even occur when the QRS duration has not shortened because of CRT [1].

In responders, CRT reduces LV dyssynchrony, measured as LV TAT or as the electrical dyssynchrony index (ED; figure 1; lowest for BiV pacing) [13]. CRT reduces the difference in the onset of electrical activation between LV and RV [21]. Ultimately, the intra- and interventricular resynchronization will improve pump function.

\section{Acute hemodynamic effects of CRT}

In dogs with $L B B B$ acute BiV pacing raises $\mathrm{dP} / \mathrm{dt}_{\max }$ almost back to pre-LBBB levels, without affecting LV end systolic or end diastolic pressures. In dogs with LBBB and tachypacing

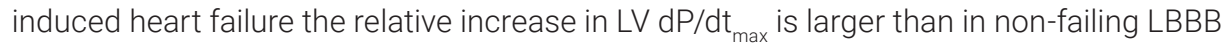
hearts, but the absolute increase is comparable [16]. In patients the onset of BiV pacing immediately increases LV $\mathrm{dP} / \mathrm{dt}_{\max }$ compared to LBBB or RV pacing and this increase typically ranges from $10-30 \%[6,7,22]$.

$\mathrm{LV} \mathrm{dP/dt}$ max has become an important variable for hemodynamic studies in CRT, but also pulse pressure and cardiac index increase during BiV stimulation [6, 22, 23]. Pressurevolume loop analysis in dogs with heart failure and LBBB shows an increase in stroke volume and stroke work in BiV pacing compared to baseline measurements [7]. It has been suggested that stroke work is a more reliable measure than increased LV $\mathrm{dP} / \mathrm{dt}_{\text {max }}$ since stroke work takes the complete systolic phase into account and comprises of both pressure and volume changes, whereas $\mathrm{LV} \mathrm{dP/dt} t_{\max }$ only reflects the isovolumic contraction 
of cardiac systole [24]. In addition, the acute improvement in $\mathrm{LV} \mathrm{dP/dt}$ max may not be able to predict the clinical outcome on the long-term [25].

\section{Long-term effects of CRT}

The favourable outcomes of CRT can be explained by reverse remodelling of the heart due to resynchronization of the ventricles. Remodelling can be activated through neurohormonal activation, differences in mechanical load and wall stress. Several studies in animal models of LBBB, sometimes in combination with tachypacing induced heart failure, have shed led light on the cellular and molecular adaptations in asynchronous hearts. The expression of some genes and proteins is depressed uniformly, while others show regional differences in expression between early- and late-activated areas. Changes occur related to myocyte function, calcium handling, $\beta$-adrenergic responsiveness, mitochondrial ATP synthase activity, cell survival signalling and other functions. CRT reverses many of these alterations, which will be reviewed in detail elsewhere in this journal.

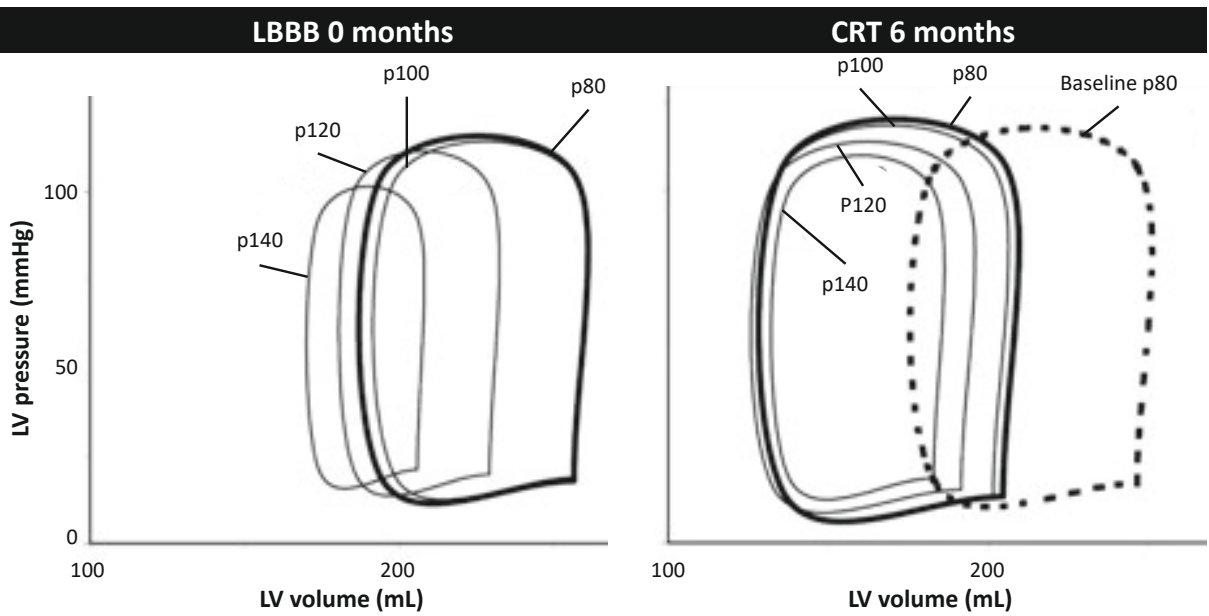

Figure 3. Pressure-volume loops at baseline and after 6 months of CRT in a patient, measured at heart rates of $80,100,120$ and 140 beats per minute. Reproduced with permission from the American Heart Association [26].

These extensive reverse-remodelling processes may also explain the improvements in pressure-volume diagrams recorded after 6 months of CRT (Figure 3). Note that after 6 months end diastolic volume and end systolic volume show a leftward shift, while ejection fraction increases [26]. Moreover, while before CRT the loops decrease in size with increased heart rate, this was much less the case after 6 months of CRT, indicating better pump function. Other studies report higher ejection fraction and lower end-systolic ventricular dimensions after one year of follow-up [24, 27]. A study in the LBBB canine model shows that acute CRT restores $\mathrm{dP} / \mathrm{dt}_{\max }$ almost back to pre-LBBB values and chronic CRT increases it slightly further, yet not completely back to pre-LBBB values [12]. 


\section{Pacing location}

The size of hemodynamic response to CRT depends on the location of the LV lead. Usually the best location is in the latest activated region of the LV free wall [28]. Intriguingly, most studies fail to indicate an anatomical location that is clearly the best. A study in LBBB dogs showed that pacing in a rather large area of the LV free wall $( \pm 43 \%)$ yielded an $\mathrm{dP}$ / $\mathrm{dt}_{\max }$ larger than $70 \%$ of the maximal observed value [29]. This, coupled with the fact that anatomy will limit the number of available pacing sites, may explain the lack of a clear anatomical defined location for pacing. Moreover, LV activation patterns vary considerably between patients. Therefore it seems advisable to estimate the ideal LV pacing region in each individual patient [28]. To this purpose, the Cardiolnsight technique (Figure 1), other electro-anatomical techniques [30], as well as echocardiographic speckle tracking [31] may be used to determine the latest activated regions.

Currently, the site of latest activation is determined during intrinsic activation, but it is probably wiser to determine it during RV apex pacing, since during CRT the RV will be paced which may shift the site of latest activation (Figure 1; compare intrinsic conduction and RV pacing) $[32,33]$.

\section{Myocardial scarring}

Myocardial scarring plays a dual role in CRT response. First, the response to CRT is inversely related to the total amount of ventricular scar tissue [35]. Secondly, CRT response is poor, and potentially adverse if the LV lead is positioned in a region with extensive scar tissue $[35,36]$. This may be related to the fact that scar tissue slows electrical conduction and may give rise to ventricular arrhythmias. However, a study in canines indicates that CRT should still be considered for patients with a myocardial scar [34]. In this study CRT reduces LV TAT and increases $\mathrm{LV} \mathrm{dP/dt}$ max to similar values in LBBB dogs with and without infarction when pacing sites are selected outside of the infarcted region (Figure 4).

\section{Multiple pacing sites}

Since BiV pacing leads to electrical resynchronization of the LV, it has been argued that increasing the number of LV pacing sites (multi-LV) will further improve resynchronization and cardiac function. The prospective TRIP-HF study shows that reverse remodelling is more pronounced after 3 months of triventricular pacing (2 LV leads) compared to conventional BiV pacing, with higher ejection fraction and lower end systolic volume in patients who did not respond to conventional BiV pacing [37]. Experiments using a strategy to maximally resynchronize the LV in the canine LBBB model show that additional pacing sites (up to seven) consistently decrease LV TAT, but only increase $\mathrm{dP} / \mathrm{dt}_{\max }$ if the hemodynamic effect of pacing the initial single-site is small (Figure 5A) [38]. Similar findings were reported later for CRT patients (Figure 5B). 


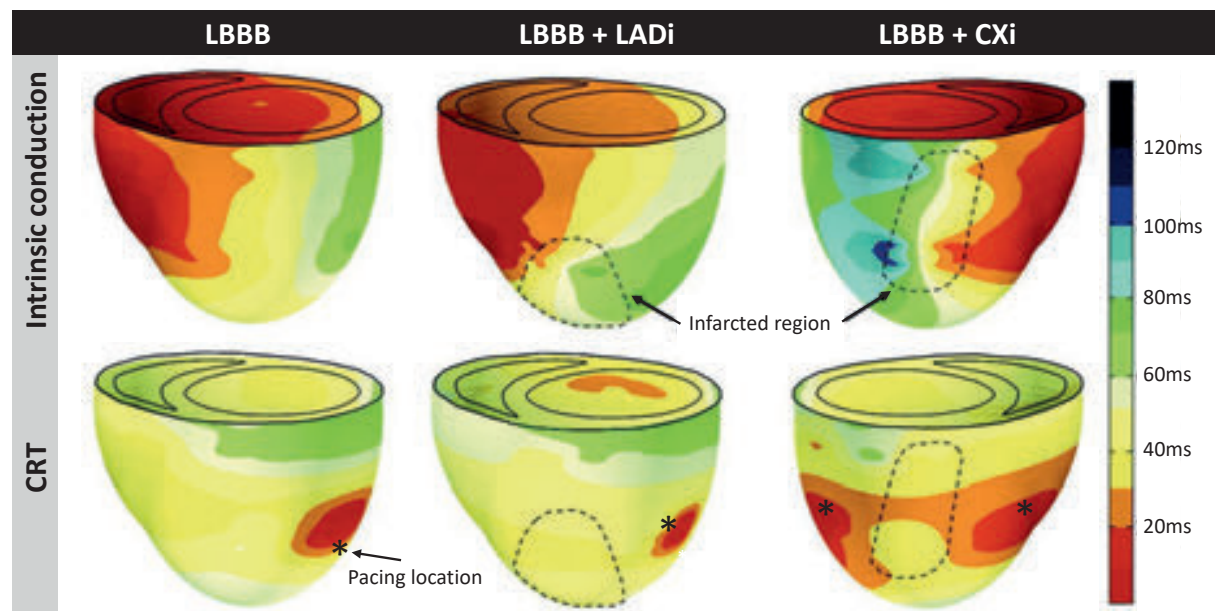

Figure 4. Three-dimensional reconstruction of electrical activation times of the LV and RV during intrinsic conduction (LBBB, top) and CRT using midlateral LV wall pacing (bottom) in representative hearts with LBBB (left), and LBBB combined with an infarction of the LAD (LBBB+LADi, middle) or left circumflex artery ( $\mathrm{LBBB}+\mathrm{LCX}$, right). Reproduced with permission from the American Heart Association [34].

While multisite pacing would often require leads in multiple veins, the recently introduced quadripolar leads enable multipoint pacing at two LV sites in one cardiac vein. The first studies on multipoint pacing have shown a small but significant increase in $\mathrm{dP} / \mathrm{dt}_{\max }$ and decrease in QRS duration compared to BiV pacing $[39,40]$. Although these results are promising, all studies so far relate to individual optimization using invasive hemodynamic measurements and no long-term benefits and clinical implications have yet been reported.

\section{Endocardial pacing}

Previous paragraphs mostly discussed (multiple) LV epicardial pacing sites, because the conventional lead position in the coronary vein is epicardial. However, both animal and patient studies indicate that LV endocardial pacing is more promising (Figure 5) [40, 41]. Several other studies corroborated these findings $[42,43]$. The better resynchronization with endocardial CRT can be explained by three factors: 1) a shorter path length for the depolarization wave to reach all regions of the ventricles, 2) more rapid impulse conduction in the endocardium than in the epicardium, and 3) a more rapid transmural conduction from endocardium to epicardium than in the opposite direction [41, 44]. Unfortunately, practical implementation of endocardial CRT is still problematic, because currently leads placed in the LV cavity require anticoagulation and also show significant dislodgement [45]. 


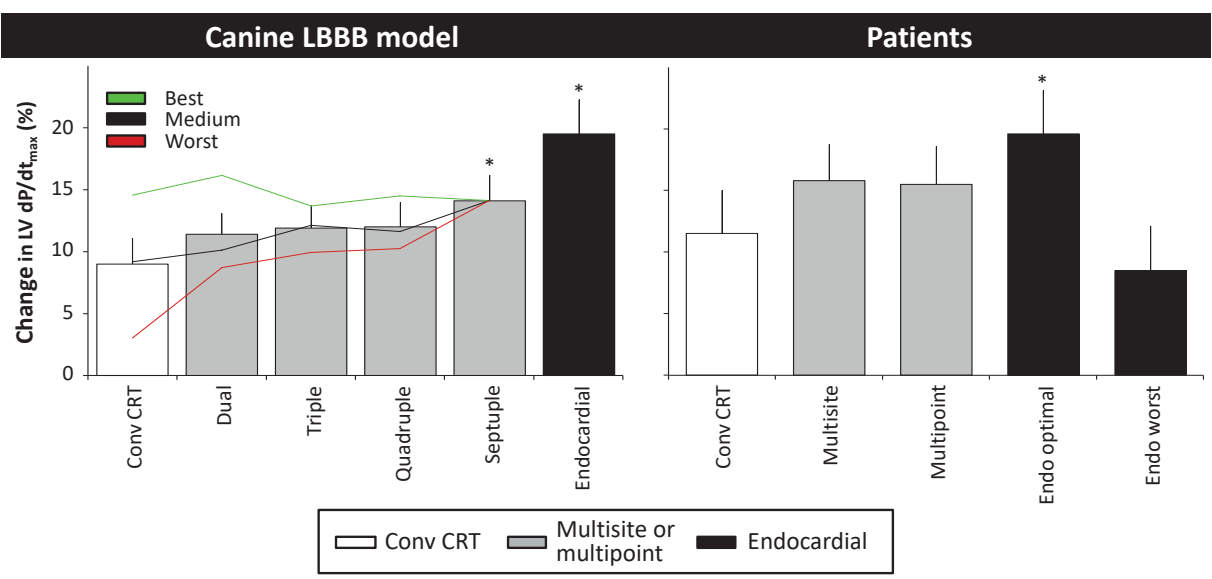

Figure 5. Percentage change in $\mathrm{LV} \mathrm{dP/dt}$ max versus baseline during conventional (Conv) CRT (white bars), multisite or multipoint (grey bars) and endocardial pacing (black bars) measured in a canine LBBB model (panel A) and in patients (panel B). The values in the bars are presented as mean+SEM. The three lines in panel A are the pooled data for the two 'worst', the three 'intermediate' and two 'best' single LV pacing sites (red, black and green, respectively). * indicates $p \leq 0.05$ vs. conventional CRT. Reproduced with permission from the Heart Rhythm Society (panel A) [38] and the European society of Cardiology (panel B) [40].

\section{Optimization of timing of pacing}

All CRT devices have the option to adjust the AV- and VV-delay to the individual patient. Figure 6A shows schematically how these different settings can influence the electrical activation of the LV. The VV-intervals ( $y$-axis) can be chosen such that either the RV or the $\mathrm{LV}$ is activated first or that both are activated simultaneously. This determines the relative contribution to the complete electrical activation of the LV by activation fronts initiated by the LV pacing site and the RV pacing site and/or intrinsic activation from the RBB. AVdelays ( $\mathrm{x}$-axis) shorter, equal to or longer than the intrinsic AV-delay influence the relative contribution of the intrinsic and paced activation wavefronts to the complete activation of the LV as well (Figure 6A). These settings affect pump function primarily by optimizing the degree of resynchronization $[12,46]$. Figure $6 \mathbf{B}$ shows the relative changes in $\mathrm{LV} \mathrm{dP/dt_{ \text {max } }}$ stroke work and electrical resynchronization in response to 100 different combinations of LV (x-axis) and RV (y-axis) AV-delays. Note the similar leftward turn (white arrows) of the optimal values for all three parameters, occurring at an RV AV-delay just shy of their respective intrinsic $\mathrm{PQ}$ duration.

Several parameters have been used to judge which AV- and VV-delays result in the best CRT-response. These include diastolic filling time, aortic velocity time integral, arterial blood pressure, LV dP/dt max stroke work and vectorcardiography $[47,48]$. However, a major problem seems to be that the increase in pump function is in the same order of magnitude as the biological variability. According to basic rules for accurate measurements, reliable results are only observed if repeated measurements are performed [49]. The fact that 
this has rarely been done may explain the variable results on the best way and clinical effect of AV-and VV-optimization. A possible solution to this problem is the use of ECG or vectorcardiogram variables for optimization, which are both easy to acquire and have considerably lower variability [50].

A

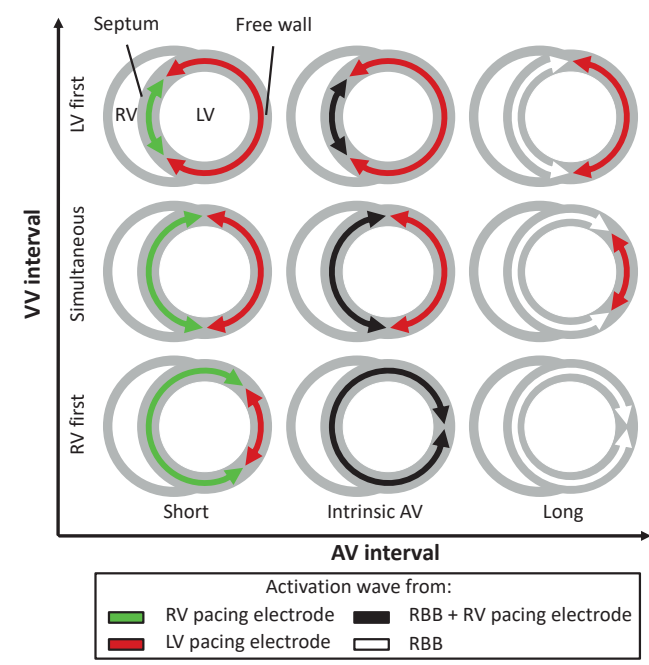

B

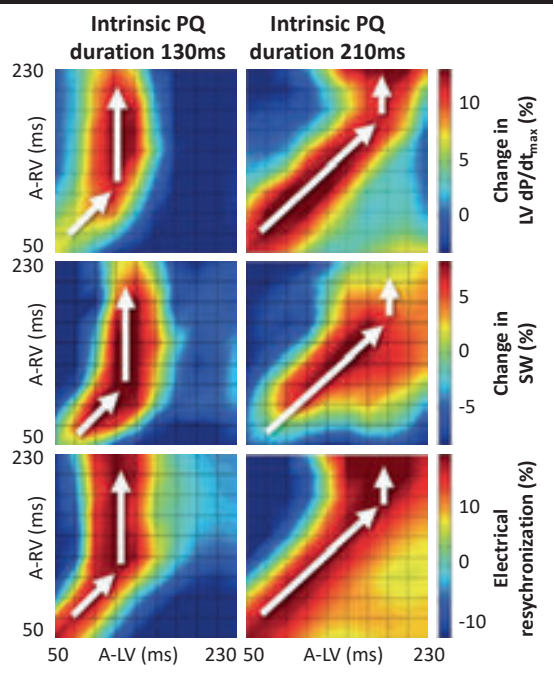

Figure 6. Changes in resynchronization and pump function after biventricular CRT. A) Schematic representation of interactions between activation wavefronts originating from the RV and LV pacing electrodes and from the right bundle branch (RBB). B) Contour plots of percentage change in LV dP/ $\mathrm{dt}_{\max }$ (maximal rate of rise of left ventricular pressure; top), stroke work (SW, middle), and electric resynchronization (bottom) in 100 different combinations of atrio-left ventricular (A-LV) and atrioright ventricular (A-RV) intervals in two different experiments with an intrinsic $P Q$ duration of 130 (left) and $210 \mathrm{~ms}$ (right). AV: atrioventricular, VV: interventricular. Panel B reproduced with permission from American Heart Association [46].

\section{CONCLUSIONS}

CRT successfully improves cardiac function in heart failure patients with LBBB, by resynchronizing the dyssynchronous contraction of the LV. Studies in patients and animals have indicated that the best electromechanical improvement is achieved by selecting the proper site for LV lead placement and by avoiding areas with transmural scarring. The acute effects of multiple pacing locations, endocardial pacing and optimized timing of pacing are superior to conventional CRT, but further research on the long-term effects of these promising approaches is still needed. 


\section{REFERENCES}

1 Verbeek XA, Vernooy K, Peschar M, Van Der Nagel T, Van Hunnik A, Prinzen FW. Quantification of interventricular asynchrony during LBBB and ventricular pacing. Am J Physiol Heart Circ Physiol. 2002;283(4):H1370-8.

2 Houthuizen P, Van Garsse LA, Poels TT, de Jaegere P, van der Boon RM, Swinkels BM, et al. Left bundle-branch block induced by transcatheter aortic valve implantation increases risk of death. Circulation. 2012;126(6):720-8.

3 Leclercq C, Kass DA. Retiming the failing heart: principles and current clinical status of cardiac resynchronization. J Am Coll Cardiol. 2002;39(2):194-201.

4 Verbeek XA, Vernooy K, Peschar M, Cornelussen RN, Prinzen FW. Intra-ventricular resynchronization for optimal left ventricular function during pacing in experimental left bundle branch block. J Am Coll Cardiol. 2003;42(3):558-67.

5 Daubert JC, Ritter P, Le Breton H, Gras D, Leclercq C, Lazarus A, et al. Permanent left ventricular pacing with transvenous leads inserted into the coronary veins. Pacing Clin Electrophysiol. 1998;21(1 Pt 2):239-45.

6 Leclercq C, Cazeau S, Le Breton H, Ritter P, Mabo P, Gras D, et al. Acute hemodynamic effects of biventricular DDD pacing in patients with end-stage heart failure. J Am Coll Cardiol. 1998;32(7):1825-31.

7 Kass DA, Chen $\mathrm{CH}$, Curry C, Talbot M, Berger R, Fetics B, et al. Improved left ventricular mechanics from acute VDD pacing in patients with dilated cardiomyopathy and ventricular conduction delay. Circulation. 1999;99(12):1567-73.

8 Brignole M, Auricchio A, Baron-Esquivias G, Bordachar P, Boriani G, Breithardt OA, et al. 2013 ESC guidelines on cardiac pacing and cardiac resynchronization therapy: the task force on cardiac pacing and resynchronization therapy of the European Society of Cardiology (ESC). Developed in collaboration with the European Heart Rhythm Association (EHRA). Europace. 2013;15(8):1070-118.

9 Abraham WT, Fisher WG, Smith AL, Delurgio DB, Leon AR, Loh E, et al. Cardiac resynchronization in chronic heart failure. N Engl J Med. 2002;346(24):1845-53.

10 Strik M, van Middendorp LB, Vernooy K. Animal models of dyssynchrony. J Cardiovasc Transl Res. 2012;5(2):135-45.

11 Liu L, Tockman B, Girouard S, Pastore J, Walcott G, KenKnight B, et al. Left ventricular resynchronization therapy in a canine model of left bundle branch block. Am J Physiol Heart Circ Physiol. 2002;282(6):H2238-44.

12 Vernooy K, Cornelussen RN, Verbeek XA, Vanagt WY, van Hunnik A, Kuiper M, et al. Cardiac resynchronization therapy cures dyssynchronopathy in canine left bundle-branch block hearts. Eur Heart J. 2007;28(17):2148-55.

13 Ghosh S, Silva JN, Canham RM, Bowman TM, Zhang J, Rhee EK, et al. Electrophysiologic substrate and intraventricular left ventricular dyssynchrony in nonischemic heart failure patients undergoing cardiac resynchronization therapy. Heart Rhythm. 2011;8(5):692-9.

14 Auricchio A, Fantoni C, Regoli F, Carbucicchio C, Goette A, Geller C, et al. Characterization of left ventricular activation in patients with heart failure and left bundle-branch block. Circulation. 2004;109(9):1133-9.

15 Lambiase PD, Rinaldi A, Hauck J, Mobb M, Elliott D, Mohammad S, et al. Non-contact left ventricular endocardial mapping in cardiac resynchronisation therapy. Heart. 2004;90(1):44-51.

16 Strik M, Regoli F, Auricchio A, Prinzen F. Electrical and mechanical ventricular activation during left bundle branch block and resynchronization. J Cardiovasc Transl Res. 2012;5(2):117-26.

17 Strik M, van Deursen CJ, van Middendorp LB, van Hunnik A, Kuiper M, Auricchio A, et al. Transseptal conduction as an important determinant for cardiac resynchronization therapy, as revealed by extensive electrical mapping in the dyssynchronous canine heart. Circ Arrhythm Electrophysiol. 2013;6(4):682-9. 
18 Helm PA, Younes L, Beg MF, Ennis DB, Leclercq C, Faris OP, et al. Evidence of structural remodeling in the dyssynchronous failing heart. Circ Res. 2006;98(1):125-32.

19 Russell K, Eriksen M, Aaberge L, Wilhelmsen N, Skulstad H, Gjesdal O, et al. Assessment of wasted myocardial work: a novel method to quantify energy loss due to uncoordinated left ventricular contractions. Am J Physiol Heart Circ Physiol. 2013;305(7):H996-1003.

20 Leyva F, Nisam S, Auricchio A. 20 years of cardiac resynchronization therapy. J Am Coll Cardiol. 2014;64(10):1047-58.

21 Liu L, Tockman B, Girouard S, Pastore J, Walcott G, KenKnight B, et al. Left ventricular resynchronization therapy in a canine model of left bundle branch block. Am J Physiol Heart Circ Physiol. 2002;282(6):H2238-44.

22 Auricchio A, Stellbrink C, Block M, Sack S, Vogt J, Bakker P, et al. Effect of pacing chamber and atrioventricular delay on acute systolic function of paced patients with congestive heart failure. The Pacing Therapies for Congestive Heart Failure Study Group. The Guidant Congestive Heart Failure Research Group. Circulation. 1999;99(23):2993-3001.

23 Gold MR, Auricchio A, Hummel JD, Giudici MC, Ding J, Tockman B, et al. Comparison of stimulation sites within left ventricular veins on the acute hemodynamic effects of cardiac resynchronization therapy. Heart Rhythm. 2005;2(4):376-81.

24 de Roest GJ, Allaart CP, Kleijn SA, Delnoy PP, Wu L, Hendriks ML, et al. Prediction of long-term outcome of cardiac resynchronization therapy by acute pressure-volume loop measurements. Eur J Heart Fail. 2013;15(3):299-307.

25 Bogaard MD, Houthuizen P, Bracke FA, Doevendans PA, Prinzen FW, Meine M, et al. Baseline left ventricular $\mathrm{dP} / \mathrm{dtmax}$ rather than the acute improvement in $\mathrm{dP} / \mathrm{dtmax}$ predicts clinical outcome in patients with cardiac resynchronization therapy. Eur J Heart Fail. 2011;13(10):1126-32.

26 Steendijk P, Tulner SA, Bax JJ, Oemrawsingh PV, Bleeker GB, van Erven L, et al. Hemodynamic effects of long-term cardiac resynchronization therapy: analysis by pressure-volume loops. Circulation. 2006;113(10):1295-304.

27 Yu CM, Chau E, Sanderson JE, Fan K, Tang MO, Fung WH, et al. Tissue Doppler echocardiographic evidence of reverse remodeling and improved synchronicity by simultaneously delaying regional contraction after biventricular pacing therapy in heart failure. Circulation. 2002;105(4):438-45.

28 Vernooy K, van Deursen CJ, Strik M, Prinzen FW. Strategies to improve cardiac resynchronization therapy. Nat Rev Cardiol. 2014;11(8):481-93.

29 Helm RH, Byrne M, Helm PA, Daya SK, Osman NF, Tunin R, et al. Three-dimensional mapping of optimal left ventricular pacing site for cardiac resynchronization. Circulation. 2007;115(8):95361.

30 Rad MM, Blaauw Y, Dinh T, Pison L, Crijns HJ, Prinzen FW, et al. Left ventricular lead placement in the latest activated region guided by coronary venous electroanatomic mapping. Europace. 2015;17(1):84-93.

31 Khan FZ, Virdee MS, Palmer CR, Pugh PJ, O'Halloran D, Elsik M, et al. Targeted left ventricular lead placement to guide cardiac resynchronization therapy: the TARGET study: a randomized, controlled trial. J Am Coll Cardiol. 2012;59(17):1509-18.

32 Mafi Rad M, Blaauw Y, Dinh T, Pison L, Crijns HJ, Prinzen FW, et al. Different regions of latest electrical activation during left bundle-branch block and right ventricular pacing in cardiac resynchronization therapy patients determined by coronary venous electro-anatomic mapping. Eur J Heart Fail. 2014;16(11):1214-22.

33 Ludwig DR, Tanaka H, Friehling M, Gorcsan J, 3rd, Schwartzman D. Further deterioration of LV ejection fraction and mechanical synchrony during RV apical pacing in patients with heart failure and LBBB. J Cardiovasc TransI Res. 2013;6(3):425-9.

34 Rademakers LM, van Kerckhoven R, van Deursen CJ, Strik M, van Hunnik A, Kuiper M, et al. Myocardial infarction does not preclude electrical and hemodynamic benefits of cardiac resynchronization therapy in dyssynchronous canine hearts. Circ Arrhythm Electrophysiol 2010;3(4):361-8. 
35 Ypenburg C, Schalij MJ, Bleeker GB, Steendijk P, Boersma E, Dibbets-Schneider P, et al. Impact of viability and scar tissue on response to cardiac resynchronization therapy in ischaemic heart failure patients. Eur Heart J. 2007;28(1):33-41.

36 Bleeker GB, Kaandorp TA, Lamb HJ, Boersma E, Steendijk P, de Roos A, et al. Effect of posterolateral scar tissue on clinical and echocardiographic improvement after cardiac resynchronization therapy. Circulation. 2006;113(7):969-76.

37 Leclercq C, Gadler F, Kranig W, Ellery S, Gras D, Lazarus A, et al. A randomized comparison of triple-site versus dual-site ventricular stimulation in patients with congestive heart failure. $\mathrm{J} \mathrm{Am}$ Coll Cardiol. 2008;51(15):1455-62.

38 Ploux S, Strik M, van Hunnik A, van Middendorp L, Kuiper M, Prinzen FW. Acute electrical and hemodynamic effects of multisite left ventricular pacing for cardiac resynchronization therapy in the dyssynchronous canine heart. Heart Rhythm. 2014;11(1):119-25.

39 Pappone C, Calovic Z, Vicedomini G, Cuko A, McSpadden LC, Ryu K, et al. Multipoint left ventricular pacing improves acute hemodynamic response assessed with pressure-volume loops in cardiac resynchronization therapy patients. Heart Rhythm. 2014;11(3):394-401.

40 Shetty AK, Sohal M, Chen Z, Ginks MR, Bostock J, Amraoui S, et al. A comparison of left ventricular endocardial, multisite, and multipolar epicardial cardiac resynchronization: an acute haemodynamic and electroanatomical study. Europace. 2014;16(6):873-9.

41 van Deursen C, van Geldorp IE, Rademakers LM, van Hunnik A, Kuiper M, Klersy C, et al. Left ventricular endocardial pacing improves resynchronization therapy in canine left bundle-branch hearts. Circ Arrhythm Electrophysiol. 2009;2(5):580-7.

42 Garrigue S, Jais P, Espil G, Labeque JN, Hocini M, Shah DC, et al. Comparison of chronic biventricular pacing between epicardial and endocardial left ventricular stimulation using Doppler tissue imaging in patients with heart failure. Am J Cardiol. 2001;88(8):858-62.

43 Bordachar P, Grenz N, Jais P, Ritter P, Leclercq C, Morgan JM, et al. Left ventricular endocardial or triventricular pacing to optimize cardiac resynchronization therapy in a chronic canine model of ischemic heart failure. Am J Physiol Heart Circ Physiol. 2012;303(2):H207-15.

44 Strik M, Rademakers LM, van Deursen CJ, van Hunnik A, Kuiper M, Klersy C, et al. Endocardial left ventricular pacing improves cardiac resynchronization therapy in chronic asynchronous infarction and heart failure models. Circ Arrhythm Electrophysiol. 2012;5(1):191-200.

45 Rademakers LM, van Gelder BM, Scheffer MG, Bracke FA. Mid-term follow up of thromboembolic complications in left ventricular endocardial cardiac resynchronization therapy. Heart Rhythm. 2014;11(4):609-13.

46 Strik M, van Middendorp LB, Houthuizen P, Ploux S, van Hunnik A, Kuiper M, et al. Interplay of electrical wavefronts as determinant of the response to cardiac resynchronization therapy in dyssynchronous canine hearts. Circ Arrhythm Electrophysiol. 2013;6(5):924-31.

47 International Working Group on Quantitative O, Sohaib SM, Whinnett ZI, Ellenbogen KA, Stellbrink C, Quinn TA, et al. Cardiac resynchronisation therapy optimisation strategies: systematic classification, detailed analysis, minimum standards and a roadmap for development and testing. Int J Cardiol. 2013;170(2):118-31.

48 van Deursen CJ, Strik M, Rademakers LM, van Hunnik A, Kuiper M, Wecke L, et al. Vectorcardiography as a tool for easy optimization of cardiac resynchronization therapy in canine left bundle branch block hearts. Circ Arrhythm Electrophysiol. 2012;5(3):544-52.

49 Whinnett ZI, Francis DP, Denis A, Willson K, Pascale P, van Geldorp I, et al. Comparison of different invasive hemodynamic methods for AV delay optimization in patients with cardiac resynchronization therapy: implications for clinical trial design and clinical practice. Int J Cardiol. 2013;168(3):2228-37.

50 van Deursen CJ, Wecke L, van Everdingen WM, Stahlberg M, Janssen MH, Braunschweig F, et al. Vectorcardiography for optimization of stimulation intervals in cardiac resynchronization therapy. J Cardiovasc Transl Res. 2015;8(2):128-37. 



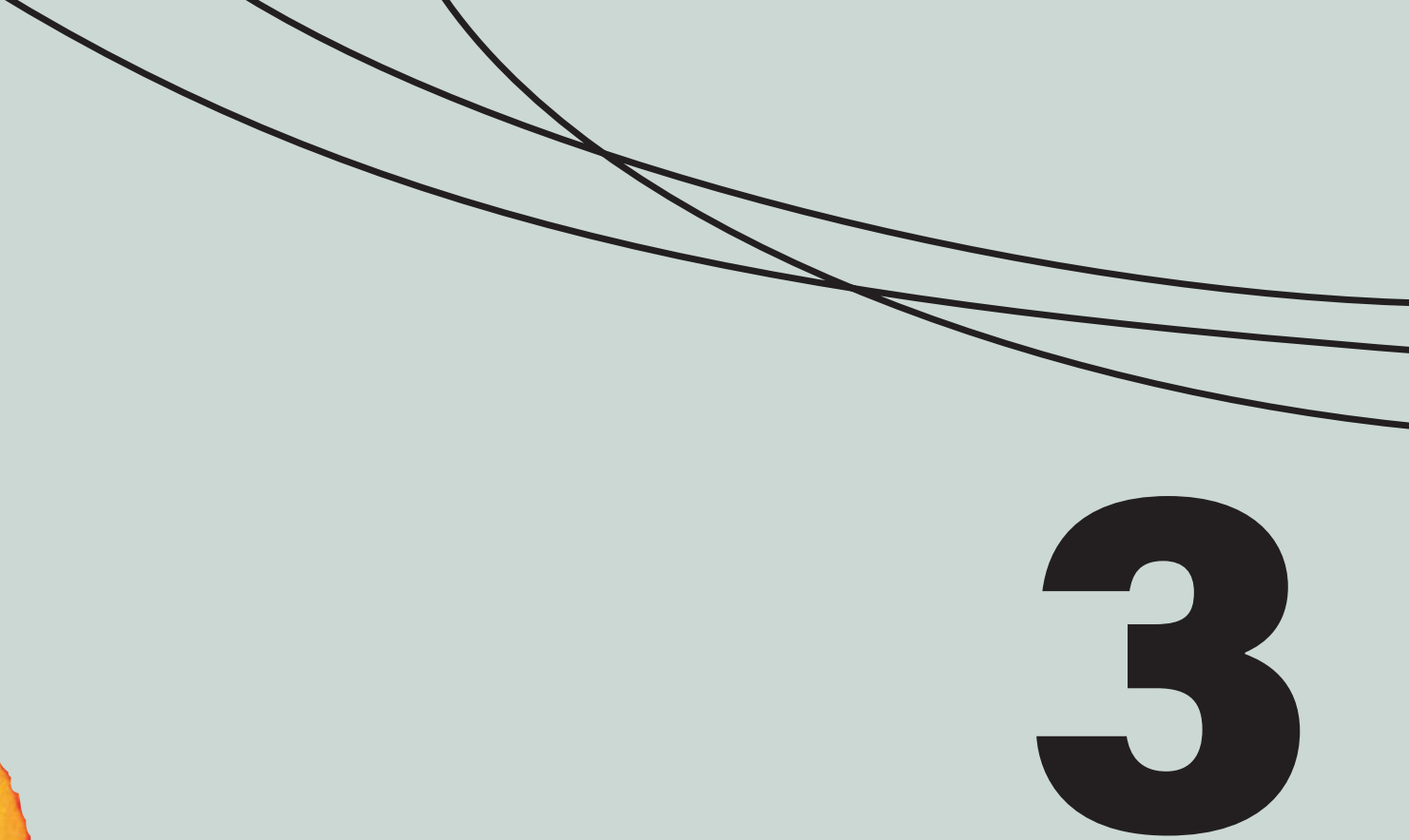

\title{
PACING THERAPY FOR ATRIOVENTRICULAR DROMOTROPATHY: A COMBINED COMPUTATIONAL-EXPERIMENTAL- CLINICAL STUDY
}

\author{
Rick Schreurs*, Floor C.W.M. Salden*, Peter R. Huntjens*, \\ Erik Willemen, Marion Kuiper, Philippe Wouters, Jos G. Maessen, \\ Pierre Bordachar, Tammo Delhaas, Justin Luermans, Mathias Meine, \\ Cornelis P. Allaart, Antonius M.W. van Stipdonk, Frits W. Prinzen, \\ Joost Lumens, Kevin Vernooy \\ ${ }^{\star}$ Authors contributed equally to this work
}




\section{ABSTRACT}

\section{Aim}

Investigate hemodynamic effects, and their mechanisms, of restoring AV-coupling using pacemaker therapy in normal and failing hearts in a combined computational-experimentalclinical study.

\section{Methods}

Computer simulations were performed in the CircAdapt model of the normal and failing human heart and circulation. Experiments were performed in a porcine model of AV dromotropathy. In a proof-of-principle clinical study, left ventricular (LV) pressure and volume were measured in 22 heart failure patients (LV ejection fraction <35\%) with prolonged PR-interval (>230ms) and narrow or non-left bundle branch block QRS complex.

\section{Results}

Computer simulations and animal studies in normal hearts showed that restoring of AVcoupling with unchanged ventricular activation sequence significantly increased LV filling, mean arterial pressure and cardiac output by $10-15 \%$. In computer simulations of failing hearts and in heart failure patients, reducing PR-interval by biventricular pacing (patients: from $300 \pm 61$ to $137 \pm 30 \mathrm{~ms}$ ) resulted in significant increases in LV stroke volume and stroke work (patients: $34 \pm 40 \%$ and $26 \pm 31 \%$, respectively). However, worsening of ventricular dyssynchrony by using right ventricular (RV) pacing abrogated the benefit of restoring AV-coupling. In model simulations, animals and patients, the increase of LV filling and associated improvement of LV pump function coincided with both larger mitral inflow (E- and A-wave area) and reduction of diastolic mitral regurgitation.

\section{Conclusions}

Restoration of AV-coupling by biventricular pacing in normal and failing hearts with prolonged AV conduction leads to a considerable hemodynamic improvement. These results indicate that biventricular or physiological pacing, but not RV pacing, may improve cardiac function in patients with heart failure and prolonged PR-interval 


\section{INTRODUCTION}

Atrioventricular (AV) conduction delay (or: AV dromotropathy), as evidenced by a prolonged PR-interval (>200ms) on the electrocardiogram (ECG), is present in $15-51 \%$ of patients with heart failure [1-4]. A prolonged PR-interval in these patients increases the risk of atrial fibrillation [5], diastolic mitral regurgitation (MR) [6] and worse clinical outcome [4]. A few small studies in the 1990s suggested that shortening the AV-delay by ventricular pacing could improve cardiac pump function [7-9]. These studies were among the first to use ventricular pacing as a treatment for heart failure. Notably, these studies employed right ventricular (RV) pacing, because these were performed before the era of biventricular (BiV) pacing. In subsequent years, the attention for treatment of a prolonged PR-interval faded as it became overruled by cardiac resynchronization therapy (CRT). However, recent sub-analyses of clinical trials investigating the benefit of CRT revitalized the interest in this topic [10]. While patients without left bundle branch block (LBBB) generally show little clinical improvement from CRT, a significant benefit was observed in non-LBBB patients with prolonged PR-interval $[11,12]$. Similarly, in a sub-study of the ReThinQ trial, which investigated the benefit of CRT in patients with QRS duration $<130 \mathrm{~ms}$, only patients with a prolonged PR-interval (>180ms) showed a significant increase in maximum oxygen uptake [13]. The 2013 ESC guidelines on pacing and CRT recommend the use of RV pacing in patients with PR-interval $>300 \mathrm{~ms}$ and find no indication to use biventricular pacing [14].

Therefore, we hypothesized that restoring proper AV-coupling by pacing significantly improves cardiac pump function. We investigated this hypothesis and revealed the mechanisms of action using a three-step approach. First, the hemodynamic benefits of restoring AV-coupling were studied in a porcine model and a computational model of the non-failing heart with prolonged PR-interval. Second, the confounding effect of pacinginduced ventricular dyssynchrony and heart failure on the potential hemodynamic benefit of restoring AV-coupling was studied in the computational model. Third, a proof-of-principle clinical study was performed in patients with heart failure and a prolonged PR-interval.

\section{METHODS}

Studies were performed in the CircAdapt computer model of the human heart and circulation, in a porcine model of AV-block and in patients with heart failure and a prolonged PR-interval (>230ms).

\section{Computer simulations}

The CircAdapt closed-loop cardiovascular system model enables real time simulation of hemodynamical and mechanical interactions between the left and right ventricles, between the atria and their distal ventricle, and between the heart and the systemic and pulmonary circulations. In CircAdapt, blood flow through the four cardiac valves is assumed to have a nonlinear flow pattern, to be unsteady and to be non-viscous $[15,16]$. Pressure 
drop across the cardiac valves is related to flow velocity through the valve and valve area (Bernoulli's principle). In addition, inertial effects due to blood mass on acceleration and deceleration are implemented in the valve module. Previously, simulations of mechanical and hemodynamic interventricular and atrio-ventricular interactions have been validated and applied under physiological and pathophysiological conditions [17-19].

\section{Reference simulations of prolonged PR with normal contractility}

Similar to the animal experiments, a reference CircAdapt simulation was created in which AV-delay was prolonged. Starting from a simulation representing the normal adult human heart, all ventricular segments were delayed by $300 \mathrm{~ms}$ relative to right atrial activation. In agreement with a prolonged pacing-induced atrial activation observed in the pigs ( $P$ width: $123 \pm 5 \mathrm{~ms}$ ), mean left atrial activation was delayed by $30 \mathrm{~ms}$ relative to mean right atrial activation. Global contractility was decreased by $20 \%$ to incorporate the reduced contractile reserve due to anaesthetics. Atrial pacing rate was set to $100 \mathrm{bpm}$, equal to the mean value in pigs $(100 \pm 2 \mathrm{bpm})$. Circulating blood volume and peripheral vascular resistance of the systemic circulation were adjusted to maintain a cardiac output of $5.1 \mathrm{~L} / \mathrm{min}$ and mean arterial pressure of $92 \mathrm{mmHg}$ in the reference simulations. The resulting values of circulating blood volume and peripheral vascular resistance were maintained during the AV-delay optimization protocol. Hemodynamic measurements of all other simulations were compared to the reference simulation.

\section{Reference simulations of prolonged PR with heart failure}

Starting from the reference simulation of prolonged PR with normal contractility, ventricular myocardial contractility was reduced to $50 \%$ of its normal value. Next, heart rate was decreased to $80 \mathrm{bpm}$ to match the heart rate in patients. Circulating blood volume and peripheral vascular resistance of the systemic circulation were adjusted to maintain a cardiac output of $4.2 \mathrm{~L} / \mathrm{min}$ and mean arterial pressure of $92 \mathrm{mmHg}$. As a result of these changes, LVEF was reduced to $34 \%$ in the heart failure reference simulation of prolonged PR.

\section{Sensitivity of hemodynamic effect by restoring AV-coupling in CircAdapt}

Similar to the animal experiment, AV-delay was decreased from $300 \mathrm{~ms}$ to $50 \mathrm{~ms}$ in CircAdapt simulations. Step size was set to $25 \mathrm{~ms}$, resulting in 10 shortened AV-delays. First, ventricular activation and atrial pacing rate were kept constant to isolate the hemodynamic effect of improving AV-coupling. Simulated hemodynamic changes induced by AV-delay shortening were compared to the measured values in animal experiments.

To isolate the effect of pacing-induced ventricular dyssynchrony on hemodynamic function, simulations with dyssynchronous ventricular activation were performed. Starting from the reference heart failure simulation with prolonged PR-interval $(300 \mathrm{~ms})$ and synchronous ventricular activation, ventricular dyssynchrony was induced concurrently with AV-delay shortening. In total, two subsets of simulations were performed with increased ventricular 
dyssynchrony as a result of pacing and compared to the simulation of synchronous activation: 1) a BiV-pacing activation pattern and 2) a RV-only pacing activation pattern. Simulated paced activation sequences were based on invasive and non-invasive electrocardiographic mapping studies during pacing [16-20].

\section{Animal experiments}

Animal handling was performed in compliance with the Guide for the Care and Use of Laboratory Animals and in accordance with the European Community recommendations. The protocol was approved by the Dutch National Ethical Committee for Animal Handling.

Experiments were performed in seven female landrace pigs weighing $61 \pm 3 \mathrm{~kg}$. Animals were pre-medicated with intramuscular Zoletil ( $5 \mathrm{mg} / \mathrm{kg}$ ). After induction with intravenous sodium thiopental (5-15 mg/kg), anesthesia was maintained by continuous infusion of Propofol (10 mg/kg/h), Sufentanyl $(5 \mu \mathrm{g} / \mathrm{kg} / \mathrm{h})$ and Rocuronium $(0.1 \mu \mathrm{g} / \mathrm{kg} / \mathrm{h})$. Details of the experimental model are provided in figure 1. Complete AV-block was created by radiofrequency ablation of the AV-node. Subsequently, the animals were paced at the right atrial appendage and at the RV apex and left ventricular (LV) epicardial lateral wall. A 7Fr conductance catheter (CD Leycom, Zoetermeer, The Netherlands) was introduced into the LV cavity via the femoral artery. A 4Fr Millar Mikro-Tip pressure catheter (Millar, Houston, Texas, USA) was used to measure left atrial (LA) pressure. A vascular flow probe (Transonic Europe B.V., Elsloo, the Netherlands) was mounted around the ascending aorta to assess aortic flow and subsequently calculate cardiac output. Measurements were performed after instrumentation and hemodynamic stabilization using BiV pacing at 10 beats per minute (bpm) above intrinsic atrial rhythm with an AV-delay of $300 \mathrm{~ms}$, mimicking prolonged PR-interval, as baseline condition. Subsequently, during BiV pacing the AV-delay was programmed between 50 and $250 \mathrm{~ms}$ in randomized steps of $50 \mathrm{~ms}$. Baseline recordings were performed before every step. Each recording lasted for at least two respiratory cycles.

\section{Patient studies}

The patient study was performed according to the principles of the Declaration of Helsinki and the study protocol was approved by the ethics committee of the Maastricht University Medical Center+ (registration number NL60764.068.17/METC 171013). All patients gave written informed consent prior to investigation, and the study was monitored by the Clinical Trial Center Maastricht. The study has been registered at clinicaltrials.gov (https:// clinicaltrials.gov/ct2/show/NCT03973944).

Patients were included in the Maastricht University Medical Center+ $(n=20)$, the University Medical Center Utrecht $(n=5)$ and the Amsterdam University Medical Center $(n=1)$ from June 2018 to February 2020. The cut-off value for PR-interval of $230 \mathrm{~ms}$ was chosen based on the subanalysis of the MADIT-CRT study [21]. Patients were implanted with a CRT-D and LV lead for this study, considering that this additional implantation creates minimal additional risk to the patient while the option to provide CRT therapy was offered after the 
hemodynamic data of this study showed significant improvement. Patients were excluded when they already had a CRT device, in the presence of a class I CRT indication (LBBB or QRS duration $>150 \mathrm{~ms}$ ). Also, a resting heart rate $>90 \mathrm{bpm}$, chronic renal failure requiring dialysis, moderate to severe aortic stenosis, frequent premature ventricular complexes ( $\geq$ two complexes on a standard ECG), significant peripheral vascular disease, an age below 18 years or recent (<3 months) myocardial infarction, coronary artery bypass grafting or valve surgery were exclusion criteria.

All participants underwent CRT device implantation according to routine clinical practice. The atrial lead was positioned in the right atrial appendage, the RV lead in the RV apical septum, and a quadripolar LV lead in a suitable vein on the posterolateral LV wall. A 7Fr pressure-volume loop conductance catheter (CD Leycom, Zoetermeer, The Netherlands) was introduced into the LV cavity via the femoral artery.

ECG and LV pressure and volume were recorded during BiV and RV pacing at four paced AV-delays. The paced AV-delay was set to approximate 100, 75, 50 and 25\% of patient's PR-interval during baseline AAI pacing minus $30 \mathrm{~ms}$ to ensure capture at the longest AVdelay. The pacing protocol (in DDD mode) was performed at $\pm 10 \mathrm{bpm}$ above intrinsic sinus rate. Interventricular pacing delay was set to -40ms (LV first). Baseline measurements were performed during atrial pacing (AAI mode) at the same pacing rate before and after each mode of ventricular pacing. Pressure-volume loops were recorded for 60 seconds during the ventricular pacing protocol and 30 seconds before and after each setting in AAI mode. The latter were averaged and are referred to as baseline [22].

\section{Data analysis}

The acute hemodynamic effect of pacing at the different AV-delays in animals and patients was evaluated by invasive measurement of LV stroke volume and stroke work (area of the pressure-volume loop) as well as the diastolic flow pattern, derived from the first derivative of the LV volume signal (flow; right panel of figure 1) using a combination of the Conduct NT software (CD Leycom, Zoetermeer, The Netherlands) and customized software programmed in MATLAB R2019b (MathWorks, Natick, Massachusetts, USA). Diastolic MR volume was quantified as the area below zero of the flow curve during diastole. Forward flow over the mitral valve was quantified as the combined area under the $\mathrm{E}$ - and A-waves (see also figure $\mathbf{1}$ and $\mathbf{2}$ ). The diastolic MR fraction was defined as diastolic MR as a percentage of forward flow. In the animals stroke volume was derived from the aortic flow probe. To account for spontaneous changes in baseline hemodynamic outcome parameters, each ventricular pacing setting was compared to the corresponding baseline. Ectopic ventricular beats and the two subsequent heart beats were excluded from the analysis. Conductance catheter measurements were volume calibrated by adjusting baseline stroke volume to stroke volume measured using Swan Ganz thermodilution catheters in animals and to preprocedural echocardiography in patients. 


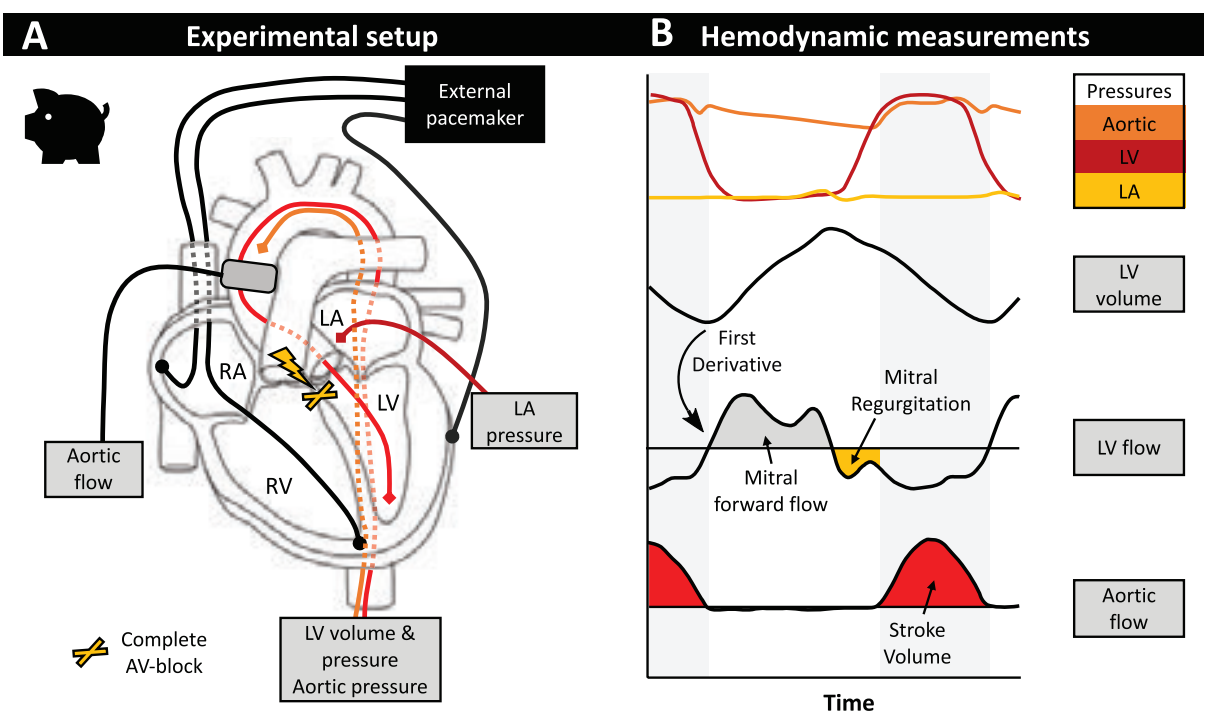

Figure 1. Schematic representation of the animal experimental set-up. (A) Overview of the porcine model. Pacemaker leads were transvenously inserted in the right atrial (RA) appendage and right ventricular (RV) apex and attached to the left ventricular (LV) epicardium. Complete atrioventricular (AV)-block was created by radiofrequency ablation of the AV-node. LV pressure and volume were measured using a conductance catheter and RV and left atrial $(L A)$ pressure were measured using a cathetertip manometer. (B) Signal analysis. The first derivative of LV volume was used to calculate forward flow over the mitral valve (grey area under E- and A-wave) and diastolic mitral regurgitation (MR, yellow area). The integral of aortic flow, measured by a flow probe, was used to quantify forward stroke volume and cardiac output (red area).

\section{Statistical analysis of clinical and experimental study}

Statistical analysis was performed using Statistical Package for Social Sciences version 24.0 (SPSS Inc., Chicago, Illinois, USA). Continuous data are presented as mean \pm standard deviation (SD). The relative change of the hemodynamic variables at various AV-delays were evaluated using a one-way repeated measures ANOVA. If significant, a Student's paired samples T-test and Bonferroni correction was used to test significance of the change at individual AV-delays. To evaluate differences between different pacing modes, two-way ANOVA for repeated measurements was used, followed by Student's paired samples T-test. A two-sided probability value of $<0.05$ was considered statistically significant. 


\section{AV-delay shortening with BiV pacing}
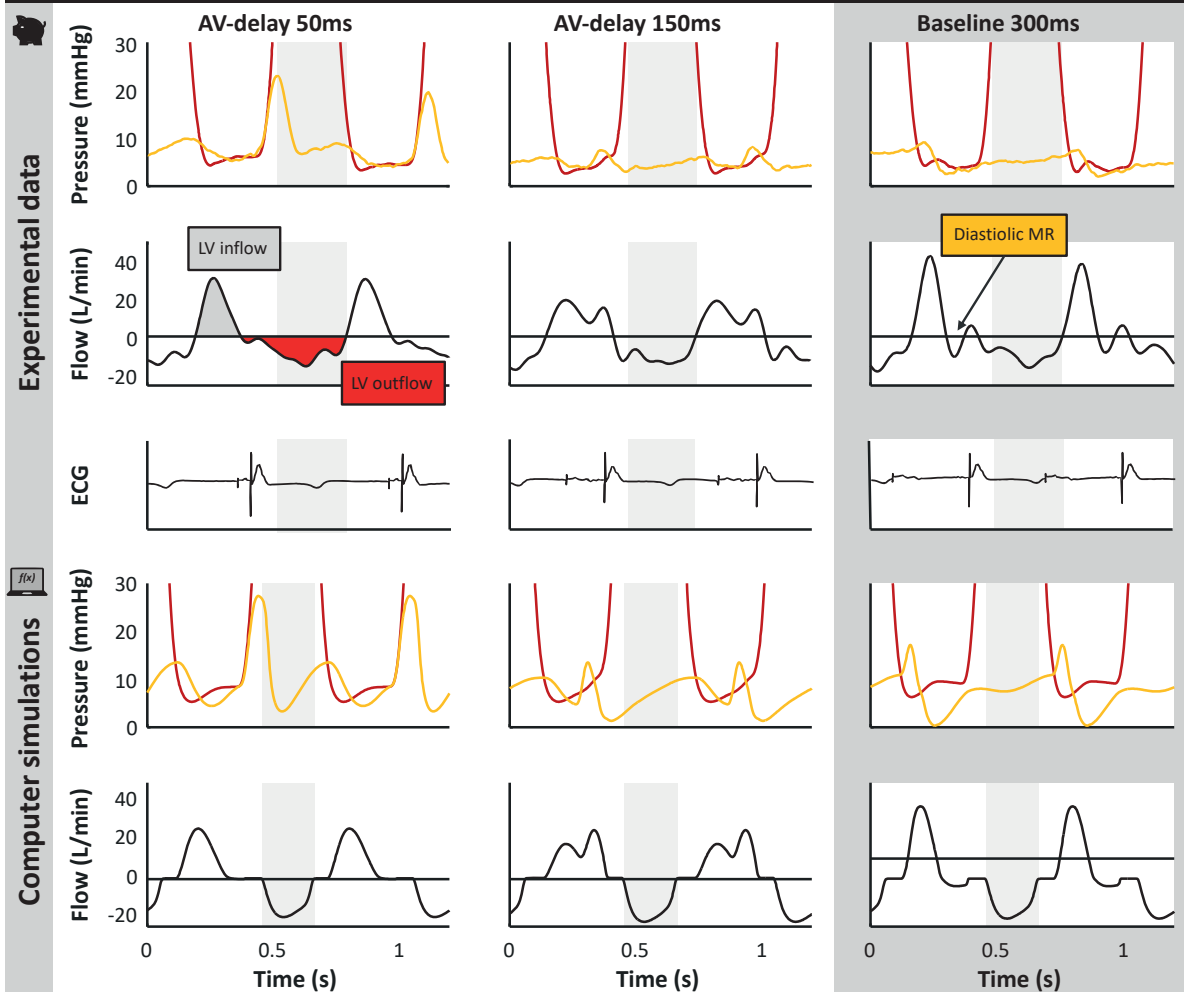

Figure 2. Hemodynamic effect of improving atrioventricular (AV)-coupling in pig experiments and computer simulations during biventricular pacing. LV and LA pressures (Top row), flow (Middle row) and electrocardiogram (Lower row) from a representative porcine experiment. Pressures and flow calculated from computer simulations (Third \& Fourth row).

\section{RESULTS}

\section{Restoring AV-coupling in normal hearts: animal experimental and computational analyses}

Results from animal experiments and computer simulations showed good qualitative and quantitative agreement (Figure 2). Under baseline conditions at long AV-delay, the delay of ventricular activation resulted in 1) suboptimal LV filling with the early filling wave (E) being fused with or prematurely interrupted by the atrial filling wave (A), and 2) diastolic MR due to atrial relaxation and related atrial pressure drop occurring before the onset of ventricular activation and, hence, papillary muscle contraction. At intermediate AV-delays (150ms) separated E- and A-waves were observed. At short AV-delays, A-wave truncation occurred as well as increases in peak and mean LA pressure, presumably caused by atrial contraction against a closed mitral valve. 
Figure 3 depicts that in the computer simulations and the animal studies, the largest increase in LV end-diastolic volume was observed at the AV-delay leading to the LV filling pattern with most pronounced E-A wave separation (175ms in the simulations, $157 \pm 7 \mathrm{~ms}$ in the animals) and leading to minimal diastolic MR. At this setting, MAP and cardiac output were also increased (both 15\% in simulations and $10 \%$ in animals). Importantly, in the animal studies the largest increase in LVEDV (at AV-delay 150ms) was achieved without a significant change in mean LA pressure compared to the baseline condition with long AV-delay (Table 1).

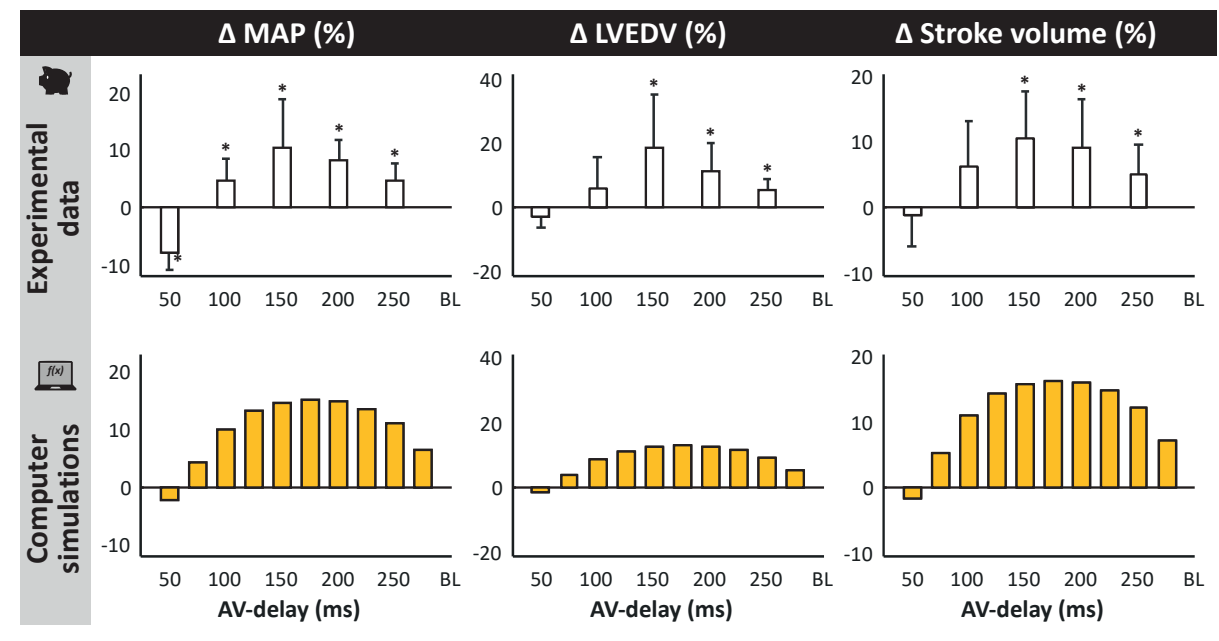

Figure 3. Hemodynamic response to improving atrioventricular (AV) coupling in pig experiments and computer simulations during biventricular pacing. Relative changes in hemodynamic function by shortening AV-delay in all pig experiments (Top panel) and simulations (Bottom panel) as compared to a baseline PR-interval of $300 \mathrm{~ms}$. MAP: mean arterial pressure, LVEDV: left ventricular end diastolic volume. For the pig experiments mean $\pm S D$ are presented. * indicates $p<0.05$ as compared to baseline.

Both the computer simulations and animal studies showed that the increased filling at intermediate AV-delays was achieved by a dual effect: larger forward flow over the mitral valve (area under the $\mathrm{E}$ - and A-waves) and a reduction in diastolic MR (Table 1).

\section{Modulating effects of ventricular dyssynchrony: computer simulations}

While the aforementioned study results concerned manipulation of AV-coupling in normal hearts at a constant degree of ventricular dyssynchrony, a next step was to investigate how different degrees of pacing-induced ventricular dyssynchrony would influence the hemodynamic response to changes of AV-coupling in the failing heart. The amount of hemodynamic improvement obtained with recovery of AV-coupling depended on the degree of pacing-induced ventricular dyssynchrony. The largest hemodynamic improvement was predicted with the simulations with synchronous ventricular activation, while RV pacing simulations showed the smallest improvement, in terms of stroke volume (Figure 4A), 
LVEDV (Figure 4B), and LV inflow pattern (Figure 4C). Most separated E- and A-waves and least diastolic MR occurred during synchronous pacing (Figure 4C).

Table 1. Hemodynamic data of AV-optimization in porcine hearts during BiV pacing

\begin{tabular}{|c|c|c|c|c|c|c|}
\hline & \multicolumn{6}{|c|}{ AV-delay } \\
\hline & $50 \mathrm{~ms}$ & $100 \mathrm{~ms}$ & $150 \mathrm{~ms}$ & $200 \mathrm{~ms}$ & $250 \mathrm{~ms}$ & $300 \mathrm{~ms}(\mathrm{BL})$ \\
\hline $\begin{array}{l}\text { PQ interval } \\
(\mathrm{ms})\end{array}$ & $53 \pm 2 *$ & $102 \pm 2 *$ & $151 \pm 1 *$ & $202 \pm 2 *$ & $254 \pm 2 *$ & $304 \pm 1$ \\
\hline MAP (mmHg) & $79 \pm 25^{\star}$ & $87 \pm 23$ & $91 \pm 24 *$ & $91 \pm 23 *$ & $88 \pm 25$ & $84 \pm 24$ \\
\hline $\begin{array}{l}\text { Cardiac output } \\
\text { aorta (L/min) }\end{array}$ & $3.0 \pm 0.3$ & $3.3 \pm 0.3$ & $3.4 \pm 0.4$ & $3.4 \pm 0.4$ & $3.2 \pm 0.3$ & $3.1 \pm 0.3$ \\
\hline $\begin{array}{l}\text { Stroke work } \\
\left(\mathrm{mmHg}{ }^{\star} \mathrm{ml}\right)\end{array}$ & $3732 \pm 1146$ & $4316 \pm 1012$ & $4437 \pm 1042$ & $4379 \pm 1123$ & $4169 \pm 1239$ & $3828 \pm 1266$ \\
\hline $\begin{array}{l}\text { LV dP/dt }{ }_{\text {max }} \\
(\mathrm{mmHg} / \mathrm{s})\end{array}$ & $1225 \pm 311 *$ & $1297 \pm 282$ & $1274 \pm 259$ & $1308 \pm 282$ & $1275 \pm 295$ & $1265 \pm 311$ \\
\hline LVSP (mmHg) & $95 \pm 21 \star$ & $102 \pm 20$ & $106 \pm 22 *$ & $105 \pm 21 *$ & $103 \pm 22$ & $99 \pm 21$ \\
\hline LV EDP $(\mathrm{mmHg})$ & $7.0 \pm 2.8$ & $8.7 \pm 2.6$ & $9.5 \pm 2.1^{\star}$ & $7.8 \pm 2.4$ & $7.4 \pm 2.9 *$ & $6.9 \pm 3.0$ \\
\hline $\begin{array}{l}\mathrm{LAP}_{\text {mean }} \\
(\mathrm{mmHg})\end{array}$ & $8.4 \pm 2.4^{\star}$ & $7.8 \pm 2.4^{\star}$ & $6.8 \pm 2.3$ & $6.6 \pm 2.2$ & $6.6 \pm 2.4$ & $6.5 \pm 2.2$ \\
\hline LV EDV $(\mathrm{mL})$ & $78 \pm 39$ & $83 \pm 39$ & $90 \pm 36 *$ & $87 \pm 36 *$ & $83 \pm 39 *$ & $80 \pm 40$ \\
\hline $\begin{array}{l}\text { Diastolic MR } \\
\text { (mL/beat) }\end{array}$ & $0.3 \pm 0.5$ & $2.5 \pm 1.9$ & $4.4 \pm 2.9$ & $4.5 \pm 3.3$ & $4.3 \pm 3.3$ & $6.1 \pm 4.0$ \\
\hline $\begin{array}{l}\text { Forward flow } \\
\text { (mL/beat) }\end{array}$ & $50 \pm 9$ & $53 \pm 8$ & $53 \pm 6$ & $53 \pm 6$ & $51 \pm 7$ & $51 \pm 8$ \\
\hline MR fraction (\%) & $0.9 \pm 1.5$ & $5.2 \pm 4$ & $9.5 \pm 6.2$ & $10 \pm 7.2$ & $10 \pm 7.7$ & $15 \pm 10.2$ \\
\hline
\end{tabular}

Results are presented as mean $\pm S D(n=7)$. BL: baseline, MAP: mean arterial pressure, LV: left ventricular,

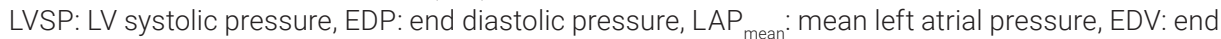
diastolic volume, ESV: end systolic volume, MR: mitral regurgitation. * indicates $\mathrm{P}<0.05$ compared to $300 \mathrm{~ms}(\mathrm{BL})$

\section{Patient study}

Table 2 shows the baseline patient characteristics. The study cohort consisted of patients with moderate to severe heart failure (NYHA II or III), mean LVEF of $29 \pm 6 \%$, mean PRinterval of $261 \pm 32 \mathrm{~ms}$, QRS duration of $123 \pm 19 \mathrm{~ms}$ and a mix of ischemic and dilated cardiomyopathy.

Similar to the computer simulations and animal studies (Figure 2 and $\mathbf{4}$ ), patients showed the characteristic pattern of E-A wave fusion and diastolic MR at baseline (Figure 5A). During BiV pacing, a clear separation of the E-and A-waves was seen at AV-delays of $50 \%$ and $75 \%$ of intrinsic PR-interval, while truncation of the A-wave occurred at shorter AV-delay (25\% of intrinsic PR-interval). Restoration of AV-coupling by BiV pacing at an AV-delay of $50 \%$ of intrinsic PR-interval $(137 \pm 30 \mathrm{~ms})$ resulted in a significant increase of forward mitral flow (on average $+8 \mathrm{~mL} /$ beat) and reduction of MR fraction (on average $-12 \%$-point, figure 5B and $\mathbf{C}$ and table 2). 


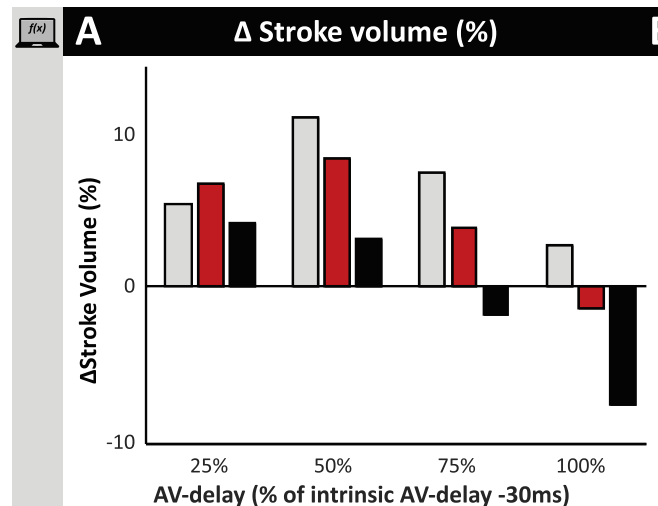

\section{B Optimal pump function}
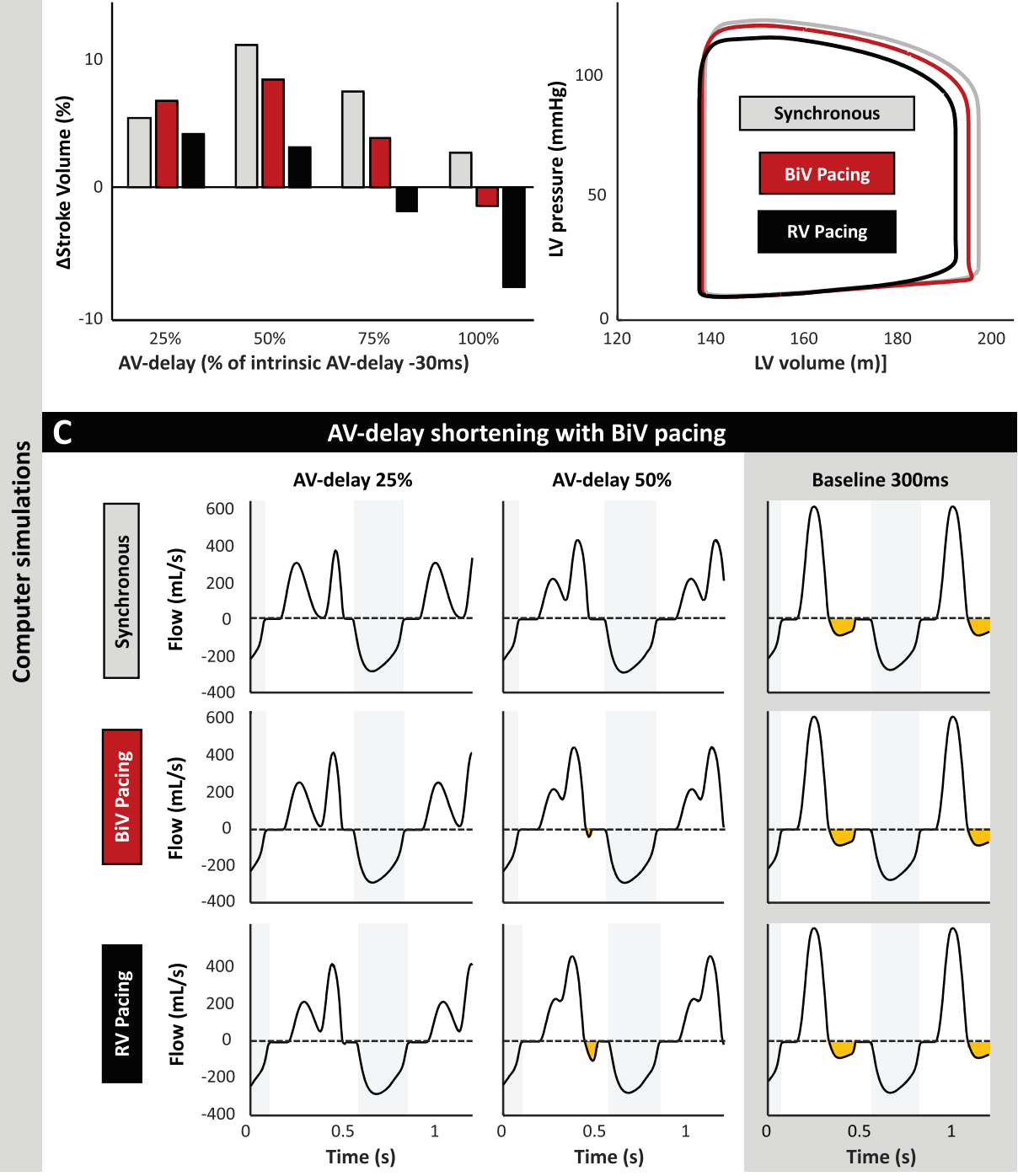

Figure 4. Effect of interventricular desynchronisation on hemodynamic benefits of restoring AV-coupling. Simulation data from the protocol where restoration of AV-coupling was achieved with unchanged synchronous ventricular activation (grey) or with biventricular (BiV) pacing (red) or right ventricular (RV) pacing (black). (A) Change in stroke volume. (B) Pressure volume loops at optimal pump function. (C) Mitral valve flow. Abbreviations as in figure $\mathbf{2}$. 
Table 2. Baseline patient characteristics

\begin{tabular}{|c|c|c|c|}
\hline Total patient no. (n) & 22 & ICM (n, \%) & $13(59)$ \\
\hline Age (years) & $67 \pm 9$ & LVEF (\%) & $29 \pm 6$ \\
\hline Men (n, \%) & $20(91)$ & \multicolumn{2}{|l|}{ Medical history (n, \%) } \\
\hline Body-mass index $\left(\mathrm{kg} / \mathrm{m}^{2}\right)$ & $29 \pm 5$ & - Myocardial infarction & $13(59)$ \\
\hline NYHA class (n, \%) & & - Prior AF & $10(46)$ \\
\hline$-\|$ & $13(59)$ & - Hypertension & $9(41)$ \\
\hline-|| $\mid$ & $9(41)$ & - CABG & $7(32)$ \\
\hline PR-interval (ms) & $261 \pm 32$ & - Diabetes mellitus & $6(27)$ \\
\hline P wave duration (ms) & $153 \pm 22$ & - COPD & $6(27)$ \\
\hline PR segment (ms) & $108 \pm 31$ & - Ischemic stroke & $5(23)$ \\
\hline QRS duration (ms) & $123 \pm 19$ & \multicolumn{2}{|l|}{ Medication (n, \%) } \\
\hline QRS morphology (n, \%) & & - Beta-blocking agents & $19(86)$ \\
\hline - IVCD & $12(55)$ & - ACE-I / ARB inhibitors ( & $19(86)$ \\
\hline - Normal & $8(36)$ & - Diuretics & $18(82)$ \\
\hline \multirow[t]{2}{*}{ - RBBB } & $2(9)$ & - Antiarrhythmic & $7(32)$ \\
\hline & & - Aldosterone antagonist & $5(23)$ \\
\hline
\end{tabular}

Results are presented as mean \pm SD or $n$ (\%). ACE-l: angiotensin-converting enzyme inhibitors, AF: atrial fibrillation, ARB: angiotensin II receptor blocker, CABG: coronary artery bypass grafting, COPD: chronic obstructive pulmonary disease, ICM: ischemic cardiomyopathy, IVCD: intraventricular conduction delay, LVEF: left ventricular ejection fraction, NYHA: New York Heart Association, PCI: percutaneous coronary intervention, RBBB: right bundle branch block.

Figure 6A presents LV pressure-volume loops of a patient during BiV (left) and RV pacing (right). As compared to baseline, BiV pacing increased LV stroke volume and stroke work (width and area of the loop, respectively), with the most pronounced benefit at an AV-delay of $50 \%$ of intrinsic PR-interval. In contrast, RV pacing tended to reduce stroke volume and stroke work, particularly at shorter AV-delays. It can also be observed that peak LV pressure diminished during RV pacing.

In the entire cohort of patients, BiV pacing increased QRS duration moderately, whereas a more pronounced prolongation occurred by applying RV pacing (Table 3). BiV pacing at an AV-delay of $50 \%$ of intrinsic PR-interval significantly increased LV stroke volume by $34 \pm 40 \%$ (Figure 6B) and LV stroke work by $26 \pm 31 \%$ (Figure $6 \mathbf{C}$ ), when compared to baseline. The increase in LV stroke work provided by BiV pacing coincided with slight but significant increases in LV end-diastolic pressure (on average $2 \mathrm{mmHg}$ ) and LV dP/dt and largely unchanged systolic LV pressure (Table 3). In contrast, restoration of the AVdelay with RV pacing did not change or even decreased stroke volume and stroke work compared to baseline (Table 3). The decrease in stroke work during RV pacing at short AV-delays coincided with significant reductions in stroke volume, systolic LV pressure and $\mathrm{LV} \mathrm{dP/dt} \mathrm{max}_{\text {max }}$ (Table 3). 
AV-delay shortening with BiV pacing
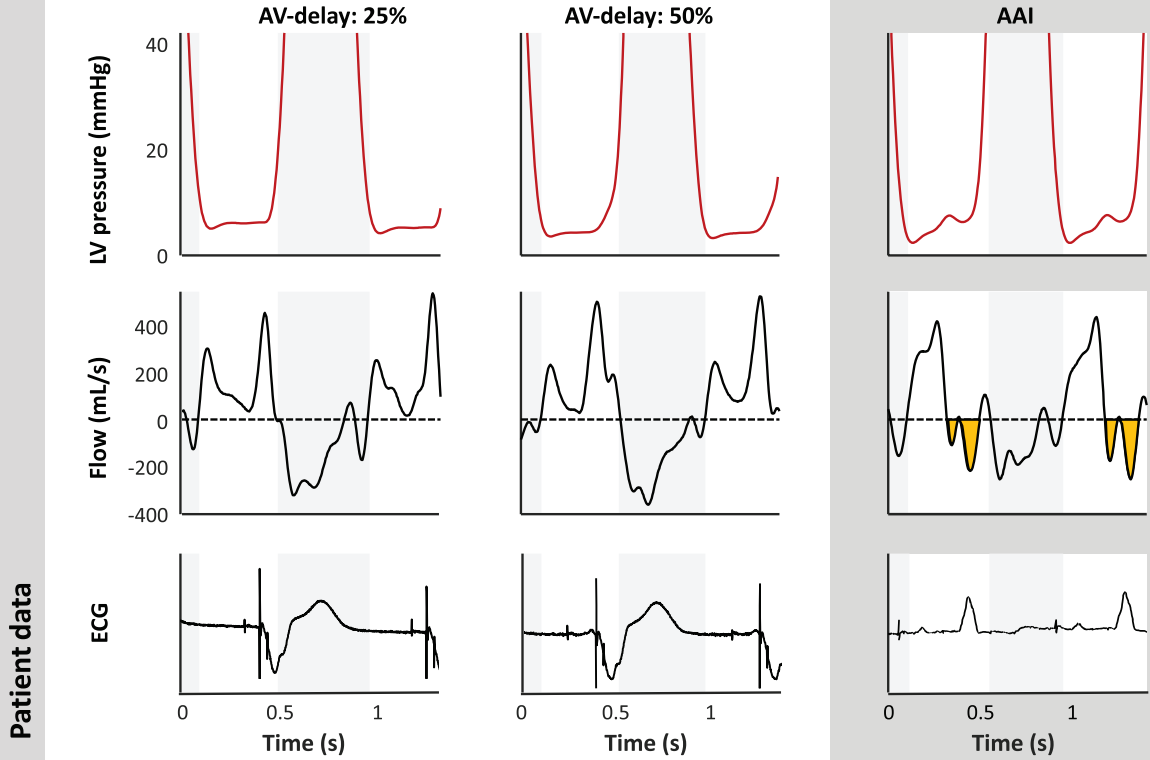

B $\Delta$ Mitral forward flow (mL/beat)

C $\Delta$ MR fraction (\%point)
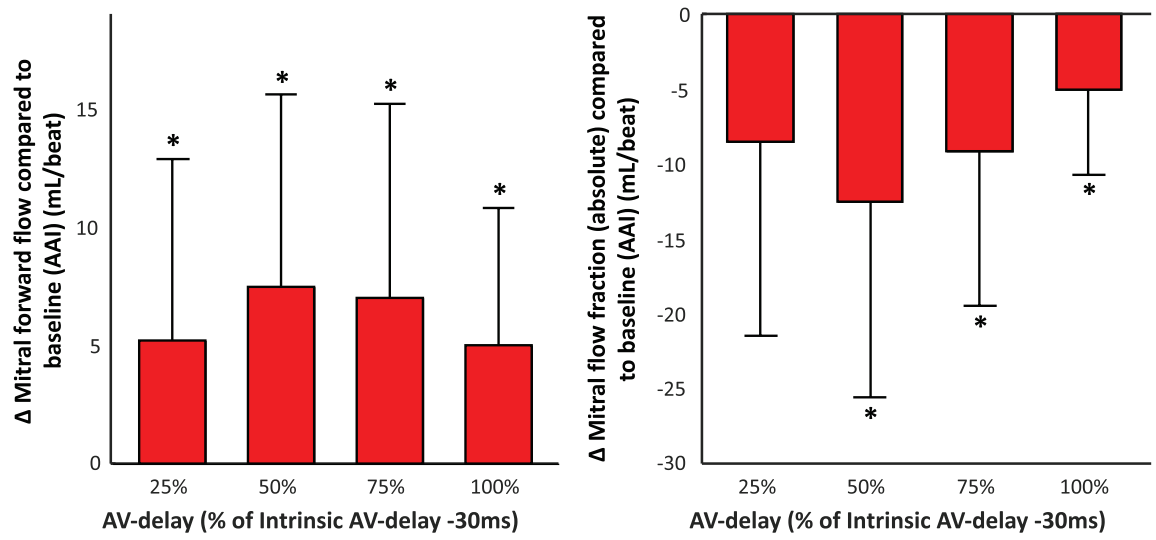

Figure 5. Hemodynamic effect of improving atrioventricular (AV)-coupling in patients. (A) Left ventricular (LV) pressure (Top row), flow (Second row) and ECG (Lowest row), as measured in a representative patient. Diastolic mitral regurgitation (MR) is presented in yellow. Absolute changes in (B) diastolic mitral forward flow and (C) diastolic MR fraction as compared to baseline (AAI) in the entire cohort. Mean $\pm S D$ are presented. * indicates $p<0.05$ as compared to baseline. 


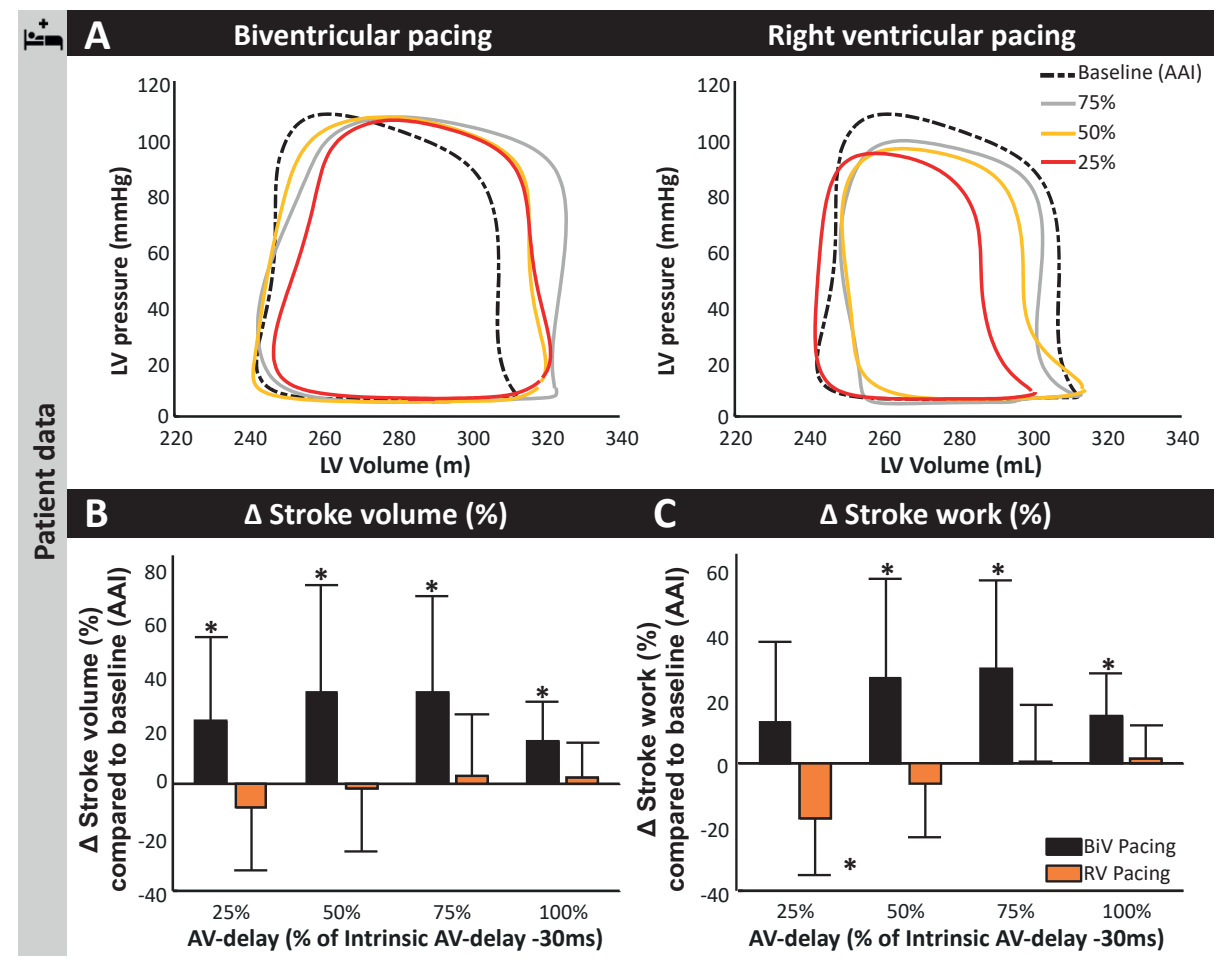

Figure 6. Hemodynamic effect of improving atrioventricular (AV)-coupling in patients during biventricular (BiV) and right ventricular (RV) pacing. (A) Left ventricular (LV) pressure-volume loops in a representative patient at baseline (dashed line) and at tested AV-delay settings (solid lines) during BiV pacing (Left) and RV pacing (Right). Relative changes in stroke volume (B) and stroke work (C) as compared to baseline (AAI) for BiV (black) and RV pacing (orange) in the entire cohort. Mean \pm SD are presented. * indicates $p<0.05$ as compared to baseline. 
Table 3. Hemodynamic and electrocardiographic data in patients paced at various paced AV-delays (\% of intrinsic PR-interval - 30ms)

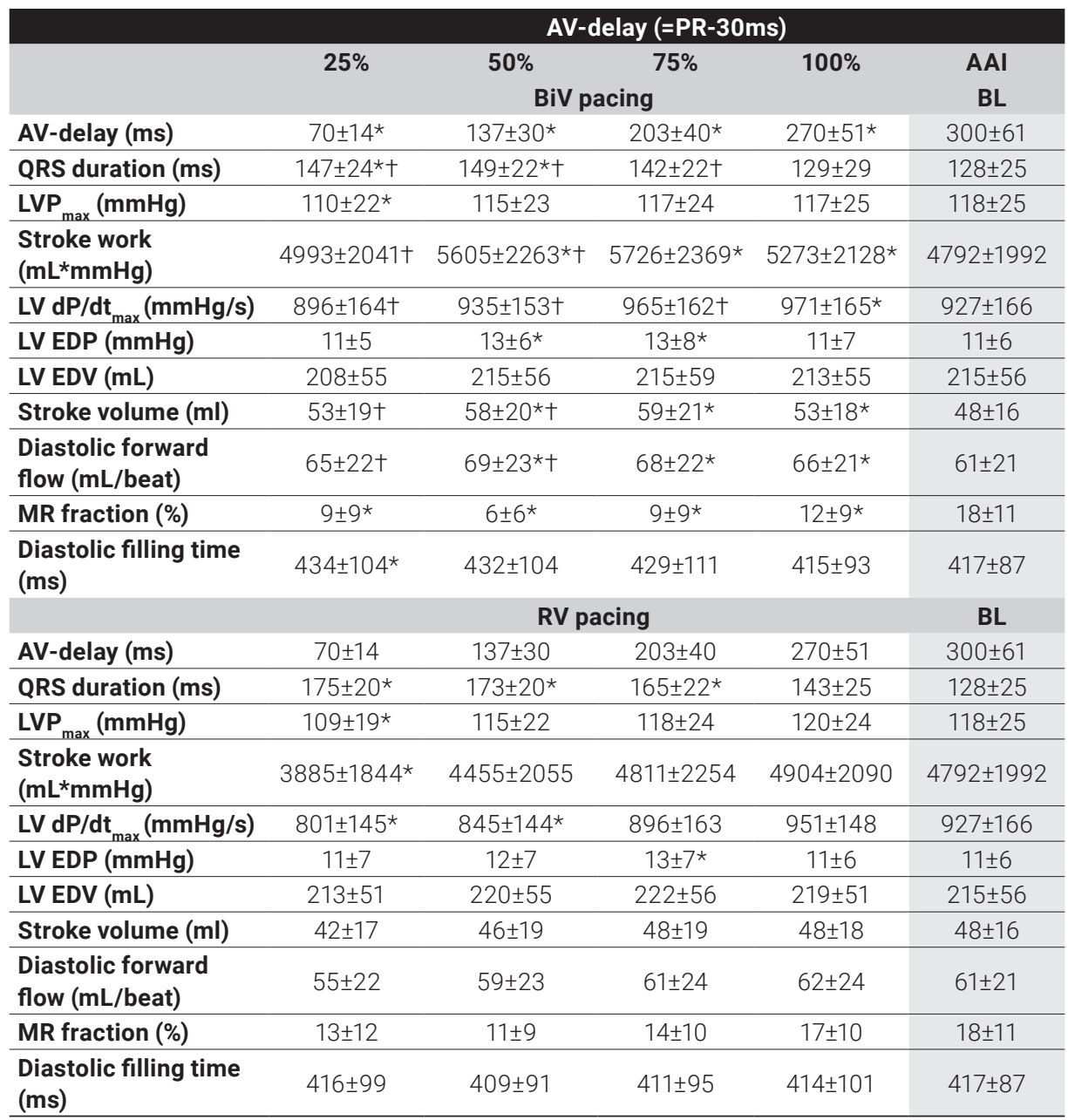

Results are presented as mean $\pm S D(n=22)$. BiV: biventricular, AV: atrioventricular, LV: left ventricular, EDP: end diastolic pressure, EDV: end diastolic volume, MR: mitral regurgitation, RV: right ventricular. * indicates $\mathrm{P}<0.05$ compared to baseline, + indicates $\mathrm{P}<0.05$ compared to RV pacing with corresponding AV-delay

\section{DISCUSSION}

The presented combination of computational, experimental and clinical proof-of-principle studies provides strong evidence that restoration of AV-coupling by BiV pacing results in significant hemodynamic benefit in hearts with AV dromotropathy (evidenced by a prolonged PR-interval). This benefit is caused by 1) increased ventricular filling, established by a larger forward flow across the mitral valve and less late-diastolic MR, but 2) is 
attenuated by ventricular desynchronization due to RV pacing. These results indicate that the 2013 guidelines [14] on pacing and CRT may need revision, because the lower limit of PR-interval for recommending pacing therapy may be decreased (from $>300 \mathrm{~ms}$ to $>230 \mathrm{~ms}$ ), whereas the use of RV pacing should be discouraged and that of biventricular pacing recommended. In order to avoid ventricular desynchronization, besides BiV pacing, also recently proposed modes of physiological ventricular pacing, such as His bundle, left bundle branch and LV septum pacing $[23,24]$ may be used to this purpose.

\section{Restoring atrioventricular coupling provides hemodynamic improvement}

A few studies in the 1990s used DDD RV pacing to restore AV-coupling [6-8]. These studies showed beneficial effects of normalization of AV-coupling in terms of reduction of diastolic MR [6,7], longer filling times and larger cardiac output [7] and higher LVEF and arterial blood pressure [8]. Notably, these studies were performed in small (12-24 patients) cohorts with variable baseline characteristics (wide and narrow QRS complex, normal and depressed cardiac function). The results from the present study not only corroborate these findings using state-of-the-art measurements, but also extend them and provide a comprehensive understanding of mechanisms involved. The complicated interaction between (intrinsic or paced) AV-delay and ventricular dyssynchrony on hemodynamics may explain why other (unpublished) studies were not able to reproduce these results, in particular when single site pacing was used.

Improving AV-coupling is an integral part of "conventional CRT" in patients with LBBB and/ or QRS duration >150ms (class I CRT indication) and its benefit can therefore be considered as evidence-based. Interestingly, recent analysis using the same computer model as used in the present study, and data from CRT patients indicated that improving AV-coupling in "conventional" CRT may be responsible for more than two-thirds of the benefit of this therapy while only one third was accounted for by ventricular resynchronization [25]. However, the feasibility, safety, and long-term efficacy of pacing-based AV-coupling in patients without LBBB and with narrow QRS complex requires further investigation in prospective clinical trials. Such studies should also provide a more precise definition of the category patients that qualify for this therapy, such as the optimal cut-off of PR-interval, degree of separation of $E$ and $A$ wave on the mitral valve Doppler velocity recording, NYHA class, ischemic or non-ischemic cardiomyopathy and LVEF. In addition, duration of the (intrinsic and paced) P-wave may be important to take into account, because large interatrial delay may safeguard LV filling in the presence of a long PR-interval.

\section{Hemodynamic improvement relates to better ventricular filling}

The crucial finding of the present study is that improving AV-coupling leads to increased LV filling, thereby increasing cardiac output at unchanged (patients) or increased (simulations, animals) blood pressure. These improvements are likely explained by the length-dependent activation of the myocardium, the cellular basis of the well-known Frank-Starling mechanism. Notably, LV dP/dt max $_{\text {ax }}$ was hardly affected, whereas this is a very sensitive 
marker of CRT benefit in LBBB patients $[26,27]$. Yet, the overall hemodynamic benefit of AV-coupling in terms of cardiac output seems at least as large as that of "CRT". Increases in stroke work, measured in this study using the conductance catheter technique, were on average slightly smaller than those measured during conventional CRT (28 vs. 43\%) [28]. However, this difference may be due to very small pressure-volume loop areas in LBBB hearts that may be associated with an artefact of the conductance catheter technique. Another important finding is that the increased LV filling during optimal AV-coupling is achieved by both improved diastolic filling pattern (i.e. larger and better separated E- and A-waves) and less diastolic MR. Finally, an important finding from the animal and simulation studies was that the improved filling was achieved while mean LA pressure was equal to or lower than baseline, indicating that the better forward pump function may even coincide with reduced backward failure.

In the past, several studies have shown similar results concerning parts of the parameters investigated in the present study. The importance of proper AV-coupling has already been addressed by animal studies in the 1960s [29,30], reporting that a properly timed effective atrial contraction is necessary for optimal LV systolic function. Similar findings were obtained in a small clinical study where echo-Doppler as well as invasive pressure and flow measurements were used. In eight patients with PR-intervals $>200 \mathrm{~ms}$, AV-optimization using DDD RV pacing increased filling times, LV end-diastolic pressure and cardiac output [31]. Other clinical studies showed echo-Doppler recordings of mitral E- and A-waves with A-wave truncation at too short AV-delays and E-A-wave fusion combined with diastolic MR at too long AV-delays [32,33].

The results from the present study provide the full picture with comprehensive invasive hemodynamic measurements in animals and patients, supplemented by computer simulations that enable control of experimental conditions that cannot be achieved in vivo. Furthermore, the use of the first derivative of the LV volume signal of the conductance catheter provides diastolic ventricular inflow patterns, rather than velocities as is the case in echo-Doppler studies. Therefore, the forward and backward flows, determined in the present study, represent the actual blood volume displaced.

The fact that the computer model could replicate all the changes seen in the animals, indicates that the mechanism of hemodynamic improvement by optimizing AV-delay can be explained by the well-established physical and physiological principles that are incorporated in the model, such as conservation of energy, inertia of blood, and lengthdependent activation of myocytes (Frank-Starling effect).

\section{Effects of ventricular pacing-induced dyssynchrony}

$\mathrm{RV}$ pacing is known to increase ventricular dyssynchrony and thereby to have a negative impact on cardiac pump function [34,35]. Our patient and simulation data show that the benefit of normalizing AV-coupling should be weighed against the detrimental effect 
of pacing-induced ventricular dyssynchrony. In the present study, BiV pacing was able to acutely increase cardiac pump function in 19 out of 22 patients, indicating that the functional gain achieved by improving AV-coupling is relatively large compared to the loss of function due to BiV pacing-induced ventricular dyssynchrony. On the other hand, severe ventricular dyssynchrony, as occurs during RV pacing abrogates the hemodynamic benefits of restoring AV-coupling. Interestingly, shortening the AV-delay using RV pacing did not improve filling either, due to a lack of reduction in diastolic MR and no increase in diastolic filling time or diastolic forward flow. These observations may be explained by a combination of factors, such as prolonged isovolumic contraction due to desynchronization and dyssynchronous contraction of the papillary muscles which increases the risk of diastolic MR. Our study also suggests that the presence of LV filling abnormalities, such as E-A wave fusion and diastolic MR, may be important selection criteria for the use of BiV pacing in patients with prolonged PR-interval.

The results from the present study may also explain the lack of benefit of algorithms aiming at minimization of ventricular pacing. After all, these algorithms do so by prolonging the AV-delay, thus inducing AV dromotropathy [36]. Our study supports the idea that too aggressive prolongation of PR-interval may have adverse effects on pump function and possibly clinical outcome.

\section{Limitations}

The present patient study has all the characteristics of a proof-of-principle study, showing acute hemodynamic effects in a small cohort. For ethical reasons, patients in this study all had the indication for ICD implant, so that the implant of the LV lead was only a minor extension of the medically indicated procedure. Clearly, studies in a wider population (also non-ICD indicated patients) and using long term outcome as endpoint are required to provide further evidence for the benefit of improving AV-coupling in patients with a prolonged PR-interval. Promising is that subanalyses in non-LBBB patients of the randomized MADIT-CRT [21], COMPANION [12] and RethinQ [13] trials also indicate that patients with long PR-interval can benefit from BiV pacing when compared to their unpaced "control group" counterparts.

The preclinical studies were performed in porcine hearts. While this species is frequently used for cardiovascular research, a limitation is that the amount of dyssynchrony induced by (single site) ventricular pacing is small. Therefore, for this study only BiV pacing was used to demonstrate the effect of AV-coupling at unchanged ventricular activation (BiV pacing being used for all AV-delays). Moreover, the lack of intrathoracic negative pressure in these open-thorax experiments may have interfered with the effect of AV-interval on filling. 


\section{CONCLUSIONS}

The combination of computational, experimental and clinical studies provides strong confirmation of previous evidence that normalizing AV-coupling by biventricular pacing in hearts with prolonged PR-interval improves cardiac pump function. This improvement is predominantly achieved by better ventricular filling, caused by a combined effect of reduction in diastolic MR and increase in diastolic forward flow. Pacing-induced ventricular dyssynchrony, caused by RV pacing, attenuates the benefit of restored AV-coupling. Therefore, this study may pave the way for a novel pacing-based therapeutic approach in patients with heart failure and prolonged PR-interval that is not part of current guidelines. 


\section{REFERENCES}

1 Nikolaidou T, Ghosh JM, Clark A. Outcomes Related to First-Degree Atrioventricular Block and Therapeutic Implications in Patients With Heart Failure. JACC Clin Electrophysiol. 2016;2(2):181-92.

2 Gervais R, Leclercq C, Shankar A, Jacobs S, Eiskjær H, Johannessen A, et al. Surface electrocardiogram to predict outcome in candidates for cardiac resynchronization therapy: a sub-analysis of the CARE-HF trial. Eur J Heart Fail. 2009;11(7):699-705.

3 Olshansky B, Day JD, Sullivan RM, Yong P, Galle E, Steinberg JS. Does cardiac resynchronization therapy provide unrecognized benefit in patients with prolonged PR intervals? The impact of restoring atrioventricular synchrony: An analysis from the COMPANION Trial. Hear Rhythm. 2012;9(1):34-9.

4 Park S-J, On YK, Byeon K, Kim JS, Choi J-O, Choi D-J, et al. Short- and long-term outcomes depending on electrical dyssynchrony markers in patients presenting with acute heart failure. Am Heart J. 2013;165(1):57-64.e2.

5 Cheng S, Keyes MJ, Larson MG, McCabe EL, Newton-Cheh C, Levy D, et al. Long-term Outcomes in Individuals with a Prolonged PR Interval or First-Degree Atrioventricular Block. JAMA. 2009;301(24):2571-7.

6 Ishikawa T, Kimura K, Miyazaki N, Tochikubo O, Usui T, Kashiwagi M, et al. Diastolic mitral regurgitation in patients with first-degree atrioventricular block. Pacing Clin Electrophysiol. 1992;15(11 Pt 2):1927-31.

7 Brecker SJ, Xiao HB, Sparrow J, Gibson DG. Effects of dual-chamber pacing with short atrioventricular delay in dilated cardiomyopathy. Lancet. 1992/11/28. 1992;340(8831):1308-12.

8 Hochleitner M, Hörtnagl H, Hörtnagl H, Fridrich L, Gschnitzer F. Long-term efficacy of physiologic dual-chamber pacing in the treatment of end-stage idiopathic dilated cardiomyopathy. Am J Cardiol. 1992;70(15):1320-5.

9 Sack S, Franz R, Dagres N, Oldenburg O, Herrmann J, Golles A, et al. Can right-sided atrioventricular sequential pacing provide benefit for selected patients with severe congestive heart failure? Am J Cardiol. 1999;83(5):124-9.

10 Salden FCWM, Kutyifa V, Stockburger M, Prinzen FW, Vernooy K. Atrioventricular dromotropathy: evidence for a distinctive entity in heart failure with prolonged PR interval? EP Eur. 2017;20(7):1067-77.

11 Kutyifa V, Stockburger M, Daubert JP, Holmqvist F, Olshansky B, Schuger C, et al. PR Interval Identifies Clinical Response in Patients With Non-Left Bundle Branch Block: A Multicenter Automatic Defibrillator Implantation Trial-Cardiac Resynchronization Therapy Substudy. Circ Arrhythmia Electrophysiol. 2014;7(4):645-51.

12 Lin J, Buhr KA, Kipp R. Effect of PR Interval on Outcomes Following Cardiac Resynchronization Therapy: A Secondary Analysis of the COMPANION Trial. J Cardiovasc Electrophysiol. 2017;28(2):185-91.

13 Joshi NP, Stopper MM, Li J, Beshai JF, Pavri BB. Impact of baseline PR interval on cardiac resynchronization therapy outcomes in patients with narrow QRS complexes: an analysis of the ReThinQ Trial. J Interv Card Electrophysiol. 2015;43(2):145-9.

14 Brignole M, Auricchio A, Baron-Esquivias G, Bordachar P, Boriani G, Breithardt OA, et al. 2013 ESC guidelines on cardiac pacing and cardiac resynchronization therapy: the task force on cardiac pacing and resynchronization therapy of the European Society of Cardiology (ESC). Developed in collaboration with the European Heart Rhythm Association . Eur Eur Pacing, Arrhythmias, Card Electrophysiol. 2013/06/27. 2013;15(8):1070-118.

15 Walmsley J, Squara P, Wolfhard U, Cornelussen R, Lumens J. Impact of abrupt versus gradual correction of mitral and tricuspid regurgitation: a modelling study. Eurolntervention. 2019;15(10):902-11. 
16 Lumens J, Fan CS, Walmsley J, Yim D, Manlhiot C, Dragulescu A, et al. Relative Impact of Right Ventricular Electromechanical Dyssynchrony Versus Pulmonary Regurgitation on Right Ventricular Dysfunction and Exercise Intolerance in Patients After Repair of Tetralogy of Fallot. J Am Hear Assoc. 2019/01/18. 2019;8(2):e010903.

17 Walmsley J, Arts T, Derval N, Bordachar P, Cochet H, Ploux S, et al. Fast Simulation of Mechanical Heterogeneity in the Electrically Asynchronous Heart Using the MultiPatch Module. PLoS Comput Biol. 2015;11(7).

18 Willemen E, Schreurs R, Huntjens PR, Strik M, Plank G, Vigmond E, et al. The left and right ventricles respond differently to variation of pacing delays in cardiac resynchronization therapy: A combined experimental-computational approach. Front Physiol. 2019;

19 Huntjens PR, Ploux S, Strik M, Walmsley J, Ritter P, Haissaguerre M, et al. Electrical Substrates Driving Response to Cardiac Resynchronization Therapy: A Combined Clinical-Computational Evaluation. Circ Arrhythmia Electrophysiol. 2018;

20 Eschalier R, Ploux S, Lumens J, Whinnett Z, Varma N, Meillet V, et al. Detailed analysis of ventricular activation sequences during right ventricular apical pacing and left bundle branch block and the potential implications for cardiac resynchronization therapy. Hear Rhythm. 2015;12(1):137-43.

21 Stockburger M, Moss AJ, Klein HU, Zareba W, Goldenberg I, Biton Y, et al. Sustained clinical benefit of cardiac resynchronization therapy in non-LBBB patients with prolonged PR-interval: MADIT-CRT long-term follow-up. Clin Res Cardiol. 2016;105(11):944-52.

22 Whinnett ZI, Nott G, Davies JER, Willson K, Manisty CH, Kanagaratnam P, et al. Maximizing efficiency of alternation algorithms for hemodynamic optimization of the AV delay of cardiac resynchronization therapy. PACE - Pacing Clin Electrophysiol. 2011;34(2):217-25.

23 Sharma PS, Vijayaraman P, Ellenbogen KA. Permanent His bundle pacing: shaping the future of physiological ventricular pacing. Nat Rev Cardiol. 2020;17(1):22-36.

24 Zhang S, Zhou X, Gold MR. Left Bundle Branch Pacing. J Am Coll Cardiol. 2019;74(24):3039-49.

25 Jones S, Lumens J, Sohaib SMA, Finegold JA, Kanagaratnam P, Tanner M, et al. Cardiac resynchronization therapy: mechanisms of action and scope for further improvement in cardiac function. Europace. 2016; euw136.

26 Kass DA, Chen CH, Curry C, Talbot M, Berger R, Fetics B, et al. Improved left ventricular mechanics from acute VDD pacing in patients with dilated cardiomyopathy and ventricular conduction delay. Circulation. 1999;99(12):1567-73.

27 Auricchio A, Stellbrink C, Block M, Sack S, Vogt J, Bakker P, et al. Effect of Pacing Chamber and Atrioventricular Delay on Acute Systolic Function of Paced Patients With Congestive Heart Failure. Circulation. 1999;99(23):2993-3001.

28 De Roest GJ, Allaart CP, Kleijn SA, Delnoy PPHM, Wu L, Hendriks ML, et al. Prediction of long-term outcome of cardiac resynchronization therapy by acute pressure-volume loop measurements. Eur J Heart Fail. 2013;15(3):299-307.

29 Skinner NS, Mitchell JH, Wallace AG, Sarnoff SJ. Hemodynamic effects of altering the timing of atrial systole. Am J Physiol Content. 1963;205(3):499-503.

30 Mitchell JH, Gupta DN, Payne RM. Influence of Atrial Systole on Effective Ventricular Stroke Volume. Circ Res. 1965;17(1):11-8.

31 Nishimura RA, Hayes DL, Holmes DR, Tajik J. Mechanism of hemodynamic improvement by dualchamber pacing for severe left ventricular dysfunction: An acute Doppler and catheterization hemodynamic study. J Am Coll Cardiol. 1995;25(2):281-8.

32 Panidis IP, Ross J, Munley B, Nestico P, Mintz GS. Diastolic mitral regurgitation in patients with atrioventricular conduction abnormalities: a common finding by Doppler echocardiography. $J$ Am Coll Cardiol. 1986;7(4):768-74.

33 Schnittger I, Appleton CP, Hatle LK, Popp RL. Diastolic mitral and tricuspid regurgitation by Doppler echocardiography in patients with atrioventricular block: New insight into the mechanism of atrioventricular valve closure. J Am Coll Cardiol. 1988;11(1):83-8. 
34 Sweeney MO, Hellkamp AS, Ellenbogen KA, Greenspon AJ, Freedman RA, Lee KL, et al. Adverse effect of ventricular pacing on heart failure and atrial fibrillation among patients with normal baseline QRS duration in a clinical trial of pacemaker therapy for sinus node dysfunction. Circulation. 2003;107(23):2932-7.

35 Curtis AB, Worley SJ, Adamson PB, Chung ES, Niazi I, Sherfesee L, et al. Biventricular Pacing for Atrioventricular Block and Systolic Dysfunction. N Engl J Med. 2013;368(17):1585-93.

36 Shurrab M, Healey JS, Haj-Yahia S, Kaoutskaia A, Boriani G, Carrizo A, et al. Reduction in unnecessary ventricular pacing fails to affect hard clinical outcomes in patients with preserved left ventricular function: a meta-analysis. Europace. 2016;19(2):282-8. 




\section{ABSTRACT}

\section{Introduction}

In pacemaker therapie the atrioventricular (AV) delay is an important determinant of ventricular filling, and therefore hemodynamic function. However, the actual AV-delay for a ventricle depends on the site of ventricular pacing and whether the right atrium is paced or sensed. Aim of this study was to examine the role of interatrial delay (IAD) and ventricular pacing site on biventricular pump function and how to assess the AV-delay that results in the best overall cardiac pump function.

\section{Methods}

Experiments were performed in 7 pigs with complete AV-block. Intracardiac pressures were measured in the right (RA) and left atrium (LA) and left (LV) and right ventricle (RV). Cardiac output was measured using an aortic flow probe. IAD was determined as time difference between $R A$ and $L A d P / d t_{\text {max }}$. AV-delay optimization protocols were performed during $L V$, $\mathrm{RV}$ and biventricular (BiV) pacing during both atrial sensing (A-S) and atrial pacing (A-P). The optimal AV-delay was defined as the AV-delay with the largest increase in cardiac output compared to AV-delay $300 \mathrm{~ms}$. Left and right effective AV-delay were defined as the time interval between LA and LV dP/dt max $_{\text {ax }}$ and between RA and RV dP/dt max $^{\prime}$ respectively. Mean effective AV-delay was calculated as the average of right and left effective AV-delay.

\section{Results}

The optimal AV-delay did not differ between the 6 pacing settings. Cardiac output increased due to a combination of increased forward flow and decreased diastolic mitral regurgitation. For all ventricular pacing sites combined, P-wave width and IAD were $\sim 40 \mathrm{~ms}$ and $10-15 \mathrm{~ms}$ larger during A-P compared to A-S, respectively. Optimal right (194 $\pm 53 \mathrm{~ms})$, left $(172 \pm 57 \mathrm{~ms})$ and mean effective AV-delay $(183 \pm 54 \mathrm{~ms})$ were significantly longer than the optimal AV-delay as programmed by the pacemaker $(155 \pm 47 \mathrm{~ms})$. The mean effective AV-delay corrected for heart rate was $27 \pm 7 \%$ and had the lowest coefficient of variance (0.27) to predict the optimal AV-delay over a wide variety of pacing modes.

\section{Conclusion}

This experimental porcine study demonstrates that IAD and interventricular dyssynchrony influence the optimal AV-delay: the optimal AV-delay increases with larger IAD (or RA preexcitation) and with LV pre-excitation. Calculation of the mean effective AV-delay allows estimation of the optimal AV-delay, regardless of atrial pacing, atrial sensing or ventricular pacing site(s). 


\section{INTRODUCTION}

The atrioventricular delay (AV-delay) is an important determinant of hemodynamic function in dual-chamber pacemaker therapy and in cardiac resynchronization therapy (CRT). Several studies have suggested that optimizing the delay between the atrial and ventricular contraction increases cardiac output by improving ventricular preload [1]. The optimal AVdelay varies from one patient to another and may change between rest and exercise [2-5].

The AV-delay plays an important role in ventricular filling. Diastolic filling exists of passive (E-wave) and active (A-wave) filling, the latter caused by atrial contraction. An AV-delay that is programmed too short leads to A-wave truncation because ventricular activation starts already before atrial contraction has finished. On the other side of the spectrum, a too long AV-delay (as in first degree AV-block) leads to fusion of the E and A-wave, which may enhance diastolic mitral regurgitation (MR). In general the optimal AV-delay is described as the shortest AV-delay without A-wave truncation [6].

It is important to realize that the AV-delay as programmed in the pacemaker is not the actual delay between activation of both atria and both ventricles. Interatrial and interventricular conduction delays may create considerable differences between right atrial-right ventricle (RA-RV) and left atrial-left ventricle (LA-LV) delays. Another important notice is that virtually all measurements of cardiac function are based on measurements in the systemic circulation. However, computer simulations in our research group emphasize that the systemic and pulmonary circulation are coupled in series, so that function of the entire circulation depends on function of both ventricles [7].

In optimization studies and in clinical practice it is known that optimal AV-delay differs substantially between atrial-sense and atrial-pace dual-chamber pacing, because atrial pacing creates a larger interatrial delay (IAD) [8-10]. As a consequence, the actual LA-LV delay is shorter than the programmed AV-delay, because the latter is based on the timing of stimulation of the RA appendage which creates a prolonged IAD. At ventricular level the pacing site is another determinant of the actual AV-delay. RV pacing leads to pre-excitation of the RV and delayed activation of the LV, while LV lateral wall pacing creates a delay in RV activation. Biventricular (BiV) pacing activates both ventricles simultaneously but changing the interventricular delay ( $\mathrm{V} V$-delay) will lead to differences in actual AV-delays between the ventricles.

Here we propose and evaluate an overarching concept of determining optimal AV-delay: the effective AV-delay (eAVD). To this purpose we used a porcine total AV-block model to examine the role of $A V$-sequential pacing during atrial pacing and atrial sensing with different ventricular pacing sites on the effective right and left AV-delay. The objectives are: 1) to explore the diastolic filling pattern during various pacing conditions, 2) to examine whether atrial sensing improves overall cardiac function compared to atrial pacing, 3) to 
achieve a better understanding of the effective right and left-sided AV-delay and 4) to find a better way of predicting the optimal paced AV-delay.

\section{METHODS}

Experiments were performed in seven female landrace pigs weighing $60.8 \pm 2.6 \mathrm{~kg}$. Animal handling was performed in compliance with the Guide for the Care and Use of Laboratory Animals and in accordance with the European Community recommendations. The protocol was approved by the Dutch National Ethical Committee for Animal Handling.

\section{Animal model}

Animals were pre-medicated with intramuscular Zoletil $(5 \mathrm{mg} / \mathrm{kg})$. After induction with intravenous sodium thiopental (5-15 mg/kg), anaesthesia was maintained by continuous infusion of Propofol (10 mg/kg/h), Sufentanyl ( $5 \mu \mathrm{g} / \mathrm{kg} / \mathrm{h})$ and Rocuronium $(0.1 \mu \mathrm{g} / \mathrm{kg} / \mathrm{h})$.

Surface electrocardiograms were recorded from the limb lead electrodes. Two pacemaker leads were transvenously inserted in the right atrial (RA) appendage and RV apex. Total AV-block was created by X-ray guided radiofrequency ablation (MarinR, Medtronic, Heerlen, the Netherlands) of the AV-node. After left-sided thoracotomy an epicardial pacemaker lead was placed on the left ventricular (LV) lateral wall. All pacemaker leads were connected to a custom-built pacemaker system. LV and RV pressures and LV volumes were measured using 7F catheter-tip manometer and conductance catheters (CD-Leycom, Zoetermeer, the Netherlands). 4F Millar Mikro-Tip pressure catheters (Millar, Houston, Texas, USA) were used to measure right and left atrial (LA) pressures. A vascular flow probe (Transonic Europe B.V., Elsloo, the Netherlands) was mounted around the ascending aorta to determine stroke volume and (after multiplication with heart rate) cardiac output.

\section{Pacing protocol}

After instrumentation and hemodynamic stabilization, the pacing protocol was performed. Six pacing protocols were performed: BiV, LV lateral wall and RV apex pacing in atrial pace $(A-P)$ and atrial sense (A-S) mode. The AV-interval was programmed between 50 and $250 \mathrm{~ms}$ in randomized steps of $50 \mathrm{~ms}$. Recordings with an AV-delay of $300 \mathrm{~ms}$ in either A-P or A-S mode were repeated after every step and the average of these measurements was considered the baseline $(\mathrm{BL})$.

\section{Data analysis}

Data analysis was performed using custom-made MATLAB software (MathWorks, Natick, Massachusetts, USA). Hemodynamic data were recorded from the first 20 seconds after switching the AV-delay. Premature beats were excluded from the analysis. PR-interval, P-wave width and QRS duration were measured from the electrocardiogram. LV and RV maximal, end systolic and end diastolic pressures were derived from the pressure curves. $\mathrm{LV}$ and $\mathrm{RV} \mathrm{dP} / \mathrm{dt}_{\max }$ were defined as the maximal rate of rise of $\mathrm{LV}$ and $\mathrm{RV}$ pressure. Mean 
arterial pressure was calculated from the aortic pressure curve. Cardiac output was calculated from the aortic flow probe signal. Optimal AV-delay $\left(A V_{\text {opt }}\right)$ was calculated as the AV-delay with the largest relative increase in cardiac output compared to the baseline value. Stroke work was quantified from pressure-volume loops. The A-waves of the LA and RA pressure curves were selected manually and subsequently maximal pressures (LAP ${ }_{\text {max }}$

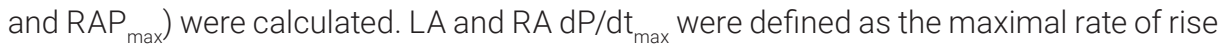
of the A-wave of the atrial pressure curve. Active and passive diastolic filling and diastolic mitral regurgitation (MR) were assessed using the first derivative of the LV volume signal of the conductance catheter. The diastolic phase was defined as the time between the

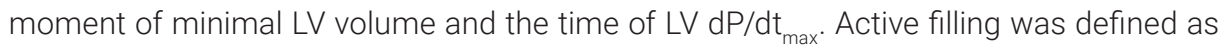
positive flow that occurred during the A-wave of the LA pressure curve. Passive filling was defined as all positive flow prior to the A-wave. Forward flow was calculated by combining passive and active filling. Diastolic MR volume was quantified as negative flow occurring after the end of active filling. See figure 1 for an overview of all hemodynamic parameters.

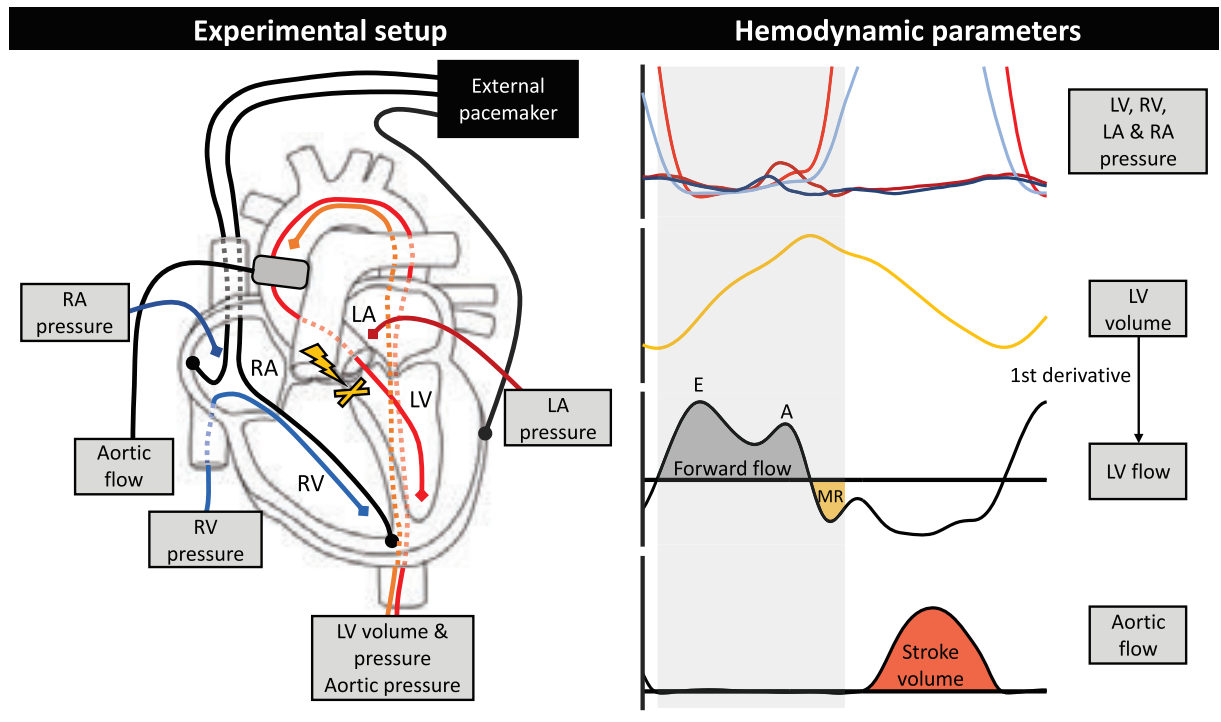

Figure 1. Experiment animal model setup and hemodynamic parameters. The left figure shows an overview of the measurement in the porcine model. Pacemaker leads were transvenously inserted in the right atrial (RA) appendage and right ventricle and epicardially on the lateral left ventricular (LV) wall. Total AV-block (indicated by the yellow cross) was induced using radiofrequency ablation of the AV-node. Aortic, LV, right ventricular (RV), left atrial (LA) and RA pressures were measured as well as $L V$ volumes. The analysis of signals is shown on the right. The first derivative of the LV volume curve was used to define forward flow over the mitral valve (grey area) and diastolic mitral regurgitation (MR, yellow area). The integral of the aortic flow, measured by a flow probe, was used to quantify forward stroke volume and cardiac output (red area). The vertical grey beam represents the diastolic phase.

Interatrial dyssynchrony was defined as the time between RA dP/dt $\max _{\max }$ and $L A d P / \mathrm{dt}_{\max }$. Interventricular dyssynchrony was quantified as the interval between the moments of $\mathrm{RV}$ and LV dP/dt ${ }_{\text {max }}$ negative values indicating RV pre-excitation. Right (right-eAVD) and 
left (left-eAVD) effective AV-delay were calculated as the interval between the times of $\mathrm{RA} d \mathrm{dP} / \mathrm{dt}_{\max }$ and $\mathrm{RV} \mathrm{dP} / \mathrm{dt}_{\max }$ and the times of $\mathrm{LA} \mathrm{dP} / \mathrm{dt}_{\max }$ and $\mathrm{LV} \mathrm{dP} / \mathrm{dt}_{\text {max }}$, respectively. The mean effective AV-delay (mean-eAVD) was defined as the interval between the mean moment of atrial $\mathrm{dP} / \mathrm{dt}_{\max }$ and the mean moment of ventricular $\mathrm{dP} / \mathrm{dt}_{\max }$. Subsequently, mean-eAVD was presented as a percentage of the RR-interval to correct for differences in heart rate (HR). The difference between right-eAVD and left-eAVD was calculated (D rightleft-eAVD), a positive value indicating that left-eAVD is shorter than right-eAVD. Figure 2 shows examples in two different situations.

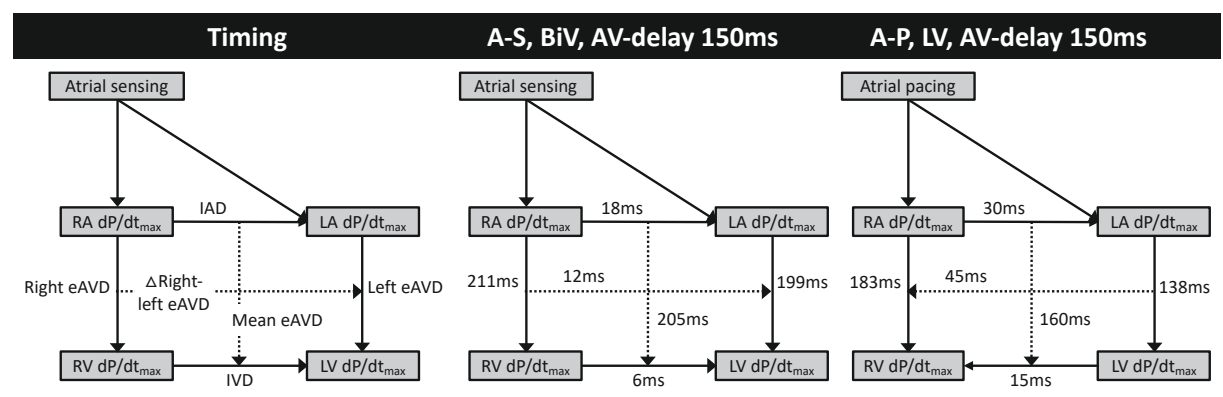

Figure 2. Examples of calculation of the effective right, left and mean AV-delay as well as the interatrial and interventricular dyssynchrony, presented for atrial sensing (A-S) BiV pacing (middle) and atrial pacing (A-P) LV pacing (right), both at an AV-delay 150ms. During A-S BiV pacing the interatrial dyssynchrony (18ms) and difference between right-eAVD (211ms) and left-eAVD (199ms) was small. A-P increased interatrial dyssynchrony (30 ms) leading to a decrease in right-eAVD (183ms), caused by the fact that atrial electrical activation was not immediately sensed. Left-eAVD (138ms) shortening was even more pronounced, caused by the delayed activation of the LA compared to the RA while the ventricles were simultaneously paced in case of BiV pacing. As a result right-eAVD was 45ms longer than left-eAVD. The arrows indicate the direction of activation. RA: right atrium, LA: left atrium, LV: left ventricle, RV: right ventricle.

\section{Statistical analysis}

Continuous data are presented as mean \pm standard error of the mean (SEM). Paired sampled t-tests were used for comparing dependent continuous variables. A two-sided probability value of $<0.05$ was considered statistically significant. Repeated measures ANOVA with Bonferroni correction were applied when different ventricular pacing sites and various AV-delays were compared. Statistics were performed using Statistical Package for Social Sciences version 26.0 (SPSS Inc., Chicago, Illinois, USA).

\section{RESULTS}

Studies were performed in 7 animals. In 1 animal the A-S protocol was not performed. Baseline hemodynamic data are presented in table 1. Cardiac output was significantly lower during A-S than during A-P in BiV, LV and RV modes, partly related to a significantly lower heart rate. In general, LV and RV end diastolic pressures were significantly higher during A-S than during A-P. RV dP/dt ${ }_{\max }$ was significantly lower in A-S mode compared to 
A-P for all ventricular pacing sites. During the A-P protocol LV dP/dt max $_{\text {was }}$ wignificantly higher during BiV pacing compared to LV and RV pacing. RAP $P_{\text {max }}$ was significantly higher during LV pacing compared to BiV and RV pacing in A-P mode.

Table 1. Hemodynamic baseline characteristics (AV-delay 300ms) for atrial pacing and atrial sensing during BiV, LV and RV pacing.

\begin{tabular}{|c|c|c|c|c|c|c|}
\hline & \multicolumn{3}{|c|}{ Atrial pacing $(\mathrm{N}=7)$} & \multicolumn{3}{|c|}{ Atrial sensing $(\mathrm{N}=6)$} \\
\hline & BiV & LV & RV & BiV & LV & RV \\
\hline HR (bpm) & $100 \pm 2$ & $100 \pm 2$ & $100 \pm 2$ & $84 \pm 3^{*}$ & $84 \pm 3 *$ & $84 \pm 3^{*}$ \\
\hline CO (L/min) & $3.1 \pm 0.1$ & $3 \pm 0.1$ & $3.1 \pm 0.1$ & $2.8 \pm 0.1^{*}$ & $2.8 \pm 0.1^{*}$ & $2.8 \pm 0.1^{\star}$ \\
\hline $\mathrm{SW}(\mathrm{mL} \cdot \mathrm{mmHg})$ & $3828 \pm 478$ & $3505 \pm 433$ & $3616 \pm 517$ & $3413 \pm 414$ & $3332 \pm 428$ & $3439 \pm 506$ \\
\hline MAP $(\mathrm{mmHg})$ & $84 \pm 9$ & $81 \pm 9^{\wedge}$ & $82 \pm 9$ & $69 \pm 7 *$ & $69 \pm 7$ & $71 \pm 7$ \\
\hline $\mathrm{LVP}_{\text {max }}(\mathrm{mmHg})$ & $99 \pm 8$ & $96 \pm 8^{\wedge}$ & $96 \pm 8$ & $87 \pm 6$ & $87 \pm 6$ & $88 \pm 6$ \\
\hline LVEDP $(\mathrm{mmHg})$ & $6.9 \pm 1.1$ & $6.6 \pm 1$ & $6.6 \pm 1$ & $8.4 \pm 1.5^{\star}$ & $8.1 \pm 1.5^{\star}$ & $8.4 \pm 1.4^{\star}$ \\
\hline LVEDV (mL) & $80 \pm 15$ & $78 \pm 14$ & $80 \pm 16$ & $87 \pm 18$ & $89 \pm 17 *$ & $88 \pm 17$ \\
\hline $\mathrm{LV} \mathrm{dP/dt}{ }_{\max }(\mathrm{mmHg} / \mathrm{s})$ & $1265 \pm 118$ & $1188 \pm 119^{\wedge}$ & $1182 \pm 110^{\wedge \S}$ & $1087 \pm 127$ & $1035 \pm 115$ & $1061 \pm 119$ \\
\hline RVESP $(\mathrm{mmHg})$ & $25 \pm 2$ & $26 \pm 1$ & $26 \pm 2$ & $25 \pm 2$ & $26 \pm 2$ & $26 \pm 2$ \\
\hline RVEDP $(\mathrm{mmHg})$ & $6.1 \pm 1.2$ & $6.2 \pm 1.2$ & $6.4 \pm 0.8$ & $8.1 \pm 1.6^{*}$ & $7.8 \pm 1.6^{\star}$ & $8.1 \pm 1.4$ \\
\hline 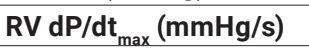 & $368 \pm 12$ & $354 \pm 22$ & $396 \pm 27$ & $318 \pm 20 *$ & $306 \pm 23^{\star}$ & $342 \pm 26^{*}$ \\
\hline $\mathrm{LAP}_{\max }(\mathrm{mmHg})$ & $11 \pm 2$ & $12 \pm 2$ & $12 \pm 2$ & $10 \pm 2$ & $10 \pm 2$ & $12 \pm 2$ \\
\hline $\operatorname{RAP}_{\text {max }}(\mathrm{mmHg})$ & $10 \pm 1$ & $12 \pm 1^{\wedge}$ & $11 \pm 1 \S$ & $9 \pm 1$ & $10 \pm 1$ & $9 \pm 1$ \\
\hline Forward flow (mL/beat) & $51 \pm 3$ & $48 \pm 2$ & $51 \pm 4$ & $53 \pm 4$ & $51 \pm 3$ & $55 \pm 4$ \\
\hline Diastolic MR (mL/beat) & $-6.1 \pm 1.5$ & $-5.7 \pm 1.4$ & $-8 \pm 2.2$ & $-6.9 \pm 2.1$ & $-6.7 \pm 1.8$ & $-9.1 \pm 2.9$ \\
\hline
\end{tabular}

Presented are mean $\pm S E M$. BiV: biventricular, LV: left ventricle, RV: right ventricle, HR: heart rate, CO: cardiac output, SW: stroke work, MAP: mean arterial pressure, LVP ${ }_{\max }$ : maximal left ventricular pressure, EDP: end diastolic pressure, EDV: end diastolic volume, $\mathrm{LAP}_{\max }$ : maximal left atrial pressure, $\mathrm{RAP}_{\max }$ : maximal right atrial pressure, MR: mitral regurgitation. * indicates $\mathrm{P}<0.05$ compared to atrial pacing for same pace site, ${ }^{\wedge}$ indicates $\mathrm{P}<0.05$ compared to BiV pacing for same atrial mode, $\S$ indicates $\mathrm{P}<0.05$ compared to LV pacing for the same atrial mode.

\section{Changes in passive and active ventricular filling during AV-delay optimization}

Figure 3 represents an example of the changes in passive (E-wave) and active (A-wave) LV filling during BiV pacing at different AV-delays. During A-P mode (upper row) at an AV-delay of $50 \mathrm{~ms}$ the A-wave was absent, because LA contraction began after the start of ventricular contraction. This led to atrial contraction against a closed mitral valve leading to high $L A$ pressure (up to $20 \mathrm{mmHg}$ ). At an AV-delay of $100 \mathrm{~ms}$ a clear distinction appeared between the E-wave and the A-wave. With increasing AV-delay the time between passive and active filling shortened, causing E and A-wave fusion at AV-delays of $150 \mathrm{~ms}$ and $200 \mathrm{~ms}$. At $200 \mathrm{~ms}$ filling was finished well before the start of ventricular contraction, leading to late diastolic MR. At the longest AV-delay of $250 \mathrm{~ms}$ no distinction between the passive and active filling could be made due to complete fusion of the $E$ and $A$-wave, resulting in a more premature stop of LV filing and larger diastolic MR. 
The diastolic filling pattern during A-S differed from A-P especially at the shortest AV-delay where LA contraction started prior to ventricular activation, which resulted in a visible A-wave and significantly lower RAP $\mathrm{m}_{\max }$ and $L A P_{\text {max }}$ (see lower row figure 3). E- and A-wave fusion seemed to happen at a shorter AV-delay and diastolic MR was present also at the shortest AV-delays. No major differences between the various ventricular pacing sites could be observed.

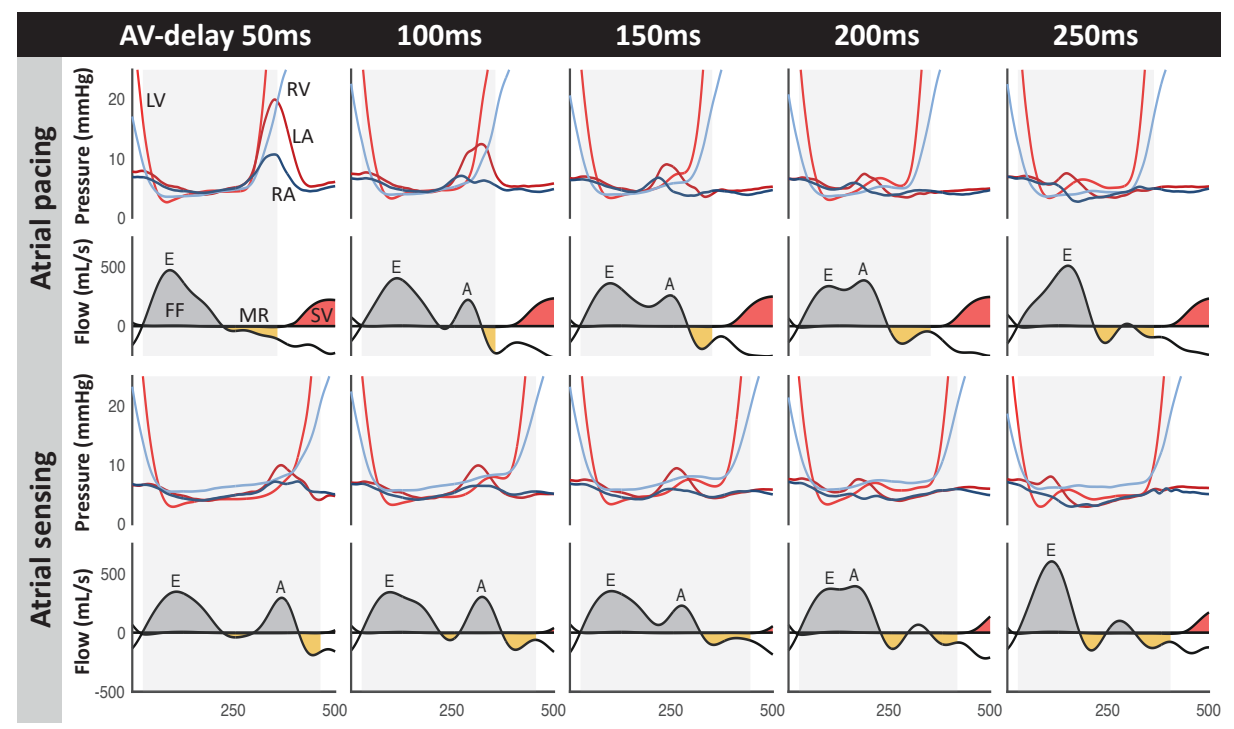

Figure 3. Typical example of changes in left ventricular filling during BiV pacing at various AV-delays in atrial pacing (upper row) and atrial sensing (lower row) mode. Left (LA) and right atrial (RA) and left (LV) and right ventricular (RV) pressures are shown. Ventricular filling (FF: forward flow) is depicted in grey and diastolic mitral regurgitation (MR) in yellow. The vertical light grey zone indicates diastole. Note the progression in diastolic MR with increasing AV-delays, the fusion of $E$ and A-wave at long AV-delays and truncation of the A-wave at short AV-delays, especially during atrial pacing at AV-delay $50 \mathrm{~ms}$.

Values of all animals for absolute changes in forward flow and diastolic MR, compared to $\mathrm{BL}$, are presented in figure 4. In A-P mode forward flow showed an increase at intermediate AV-delays and decreased at AV-delay 50ms compared to BL values. Also, shortening AV-delay decreased diastolic MR, which was largest at the shortest AV-delays and less pronounced when AV-delays were longer than $100 \mathrm{~ms}$. No significant differences were found between the various ventricular pacing sites. Also, the patterns during A-S were comparable to A-P mode. 


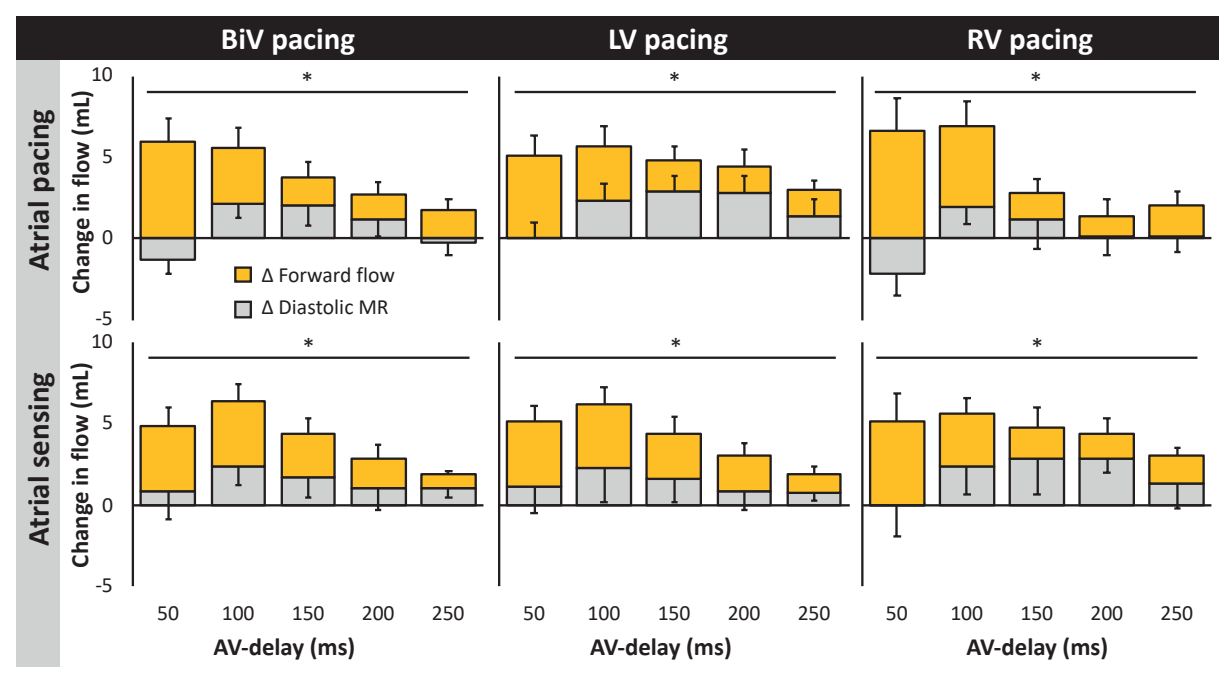

Figure 4. Absolute change in forward flow (red bars) and diastolic mitral regurgitation (grey bars) at various AV-delays during BiV (left), LV (middle) and RV (right) pacing in atrial pace (upper) and atrial sense (lower) mode, compared with their contribution at AV-delay $300 \mathrm{~ms}$. A decrease in mitral regurgitation is presented as positive bars since it leads to an increase in stroke volume. Values are presented as mean $\pm S E M$, * indicates $\mathrm{P}<0.05$ using repeated measures ANOVA.

\section{Hemodynamic effect of atrioventricular optimization}

AV-delay optimization resulted in a parabolic curve for cardiac output and stroke work for all six pacing conditions (A-P and A-S with BiV, LV and RV pacing, see figure 5). During A-S the peak of the parabolic curve tended to occur at shorter AV-delays (142-150ms) than during A-P (157-171ms). In table 2 the hemodynamic parameters at the optimal AV-delay $\left(A V_{\text {opt }}\right)$ are presented. All six pacing modes achieved a significant increase in cardiac output compared to $B L$ values, ranging from $+10.7 \%$ to $+14.2 \%$. Furthermore stroke work, mean arterial pressure, maximal LV pressure and RV end systolic pressure were significantly higher at $\mathrm{AV}_{\text {opt }}$ compared to the $\mathrm{BL}$ value during all pacing modes. Ventricular filling was improved at $A V_{\text {opt }}$ as evidenced by a significant increase in LV end diastolic volume in 5 out of 6 settings and in LV end diastolic pressure in 4 out of 6 settings. Interestingly, the increases in cardiac output and cardiac pressures occurred in the absence of a significant increase of LV and RV dP/dt max $_{\text {ax }}$ (table 2). Diastolic MR tended to decrease in all settings, but was significantly lower compared to BL only in BiV and LV pacing in A-S mode. 


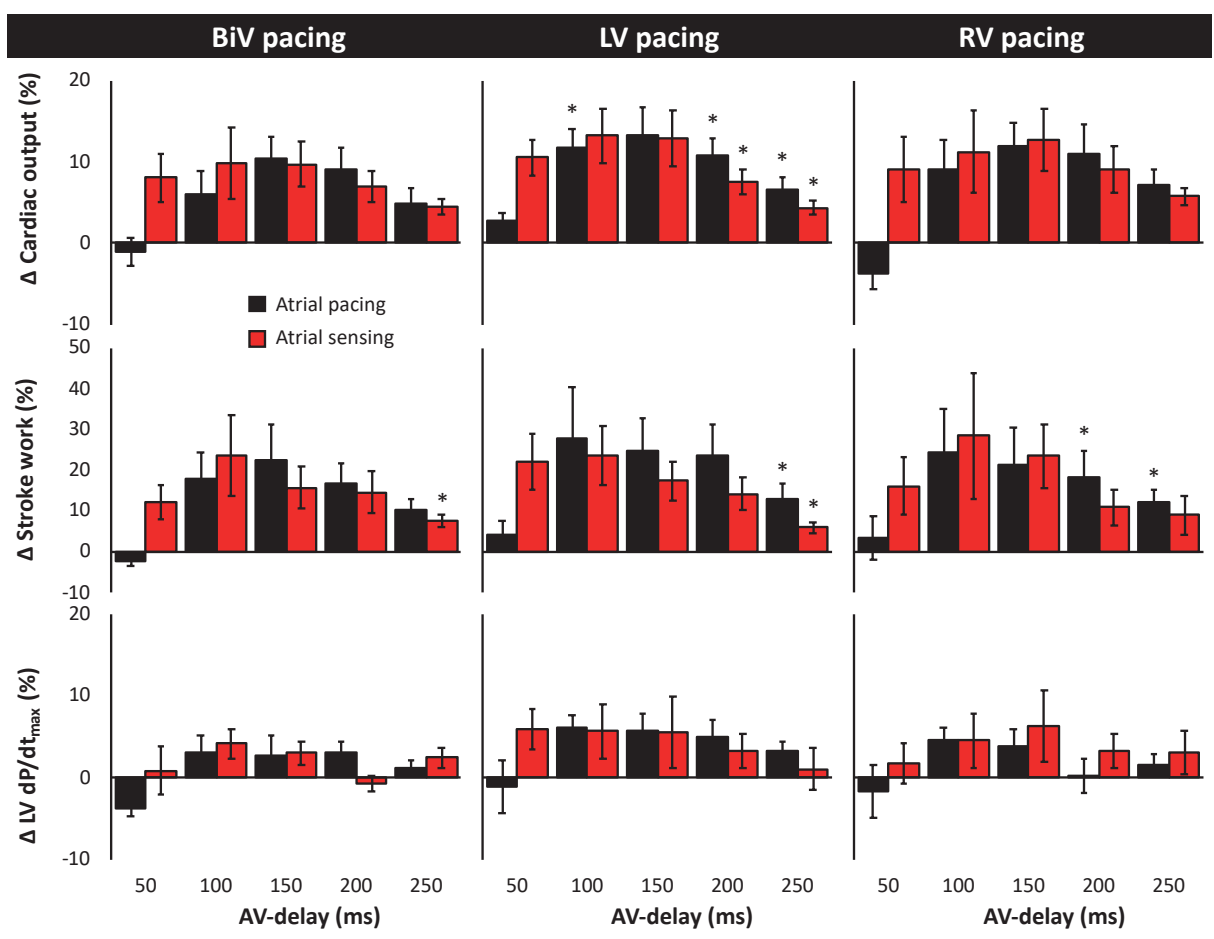

Figure 5. Changes of cardiac output (upper panels), stroke work (middle panels) and LV dP/dtmax (lower panels) during BiV (left panels), LV (middle panels) and RV (right panels) pacing mode while atrial pacing (black bars) and atrial sensing (yellow bars). Data are presented as \%change relative to the corresponding pacing mode with an AV-delay of $300 \mathrm{~ms}$. * indicates $\mathrm{P}<0.05$ compared to AV-delay $300 \mathrm{~ms}$ using paired samples T-test with Bonferroni correction.

\section{Effect of different pacing modes on hemodynamic parameters}

The optimal AV-delay did not differ between the various ventricular pacing sites and seemed to be shorter in A-S compared to A-P though not significantly. The hemodynamic improvement by AV optimization (as evidenced by \%change in cardiac output, stroke work and mean arterial pressure) was not significantly different between A-P and A-S for any ventricular pacing site. The decrease of diastolic MR was significantly more during LV pacing in A-S mode compared to A-P $(-3.5 \mathrm{~mL} \pm 2.4$ vs $-2 \mathrm{~mL} \pm 2.5, \mathrm{P}<0.05$, see table 2).

No significant differences were present between the various ventricular pacing sites for A-S mode. In A-P mode the decrease in $\mathrm{RAP}_{\max }$ was significantly bigger during LV pacing compared to BiV and RV pacing (table 2). 
Table 2. Changes in hemodynamic parameters at optimal AV-delay based on maximal relative increase in cardiac output for each pace setting compared to the baseline setting with AV-delay $300 \mathrm{~ms}$.

\begin{tabular}{|c|c|c|c|c|c|c|}
\hline & \multicolumn{3}{|c|}{ Atrial pacing } & \multicolumn{3}{|c|}{ Atrial sensing } \\
\hline & BiV & LV & RV & BiV & LV & RV \\
\hline$A V_{o p t}(m s)$ & $157 \pm 7 *$ & $171 \pm 18^{*}$ & $157 \pm 13^{*}$ & $150 \pm 29 *$ & $142 \pm 24 *$ & $150 \pm 22^{*}$ \\
\hline$\Delta \mathrm{CO}(\%)$ & $10.7 \pm 6.9 *$ & $13.3 \pm 8.7 *$ & $12.2 \pm 8.8^{\star}$ & $12.2 \pm 8.7^{\star}$ & $12.9 \pm 8.2^{\star}$ & $14.2 \pm 11.3^{*}$ \\
\hline$\Delta \mathrm{SW}(\%)$ & $2 \pm 24.1^{\star}$ & $4.1 \pm 22.6^{*}$ & $23.5 \pm 28.4^{\star}$ & $21.8 \pm 25.1$ & $21.7 \pm 18.9 *$ & $32.5 \pm 39.5^{\star}$ \\
\hline$\triangle$ MAP (\%) & $9.3 \pm 8.4^{\star}$ & $10.3 \pm 5.6^{\star}$ & $10.1 \pm 4.5^{\star}$ & $10.9 \pm 10.3 *$ & $10.4 \pm 4.5^{\star}$ & $14 \pm 10.8 *$ \\
\hline$\Delta$ LVP $_{\max }(\%)$ & $7 \pm 5.2^{\star}$ & $7.7 \pm 4.3^{\star}$ & $8.3 \pm 3.5^{\star}$ & $7.3 \pm 5.9 *$ & $7.1 \pm 2.8^{*}$ & $10 \pm 7.3^{*}$ \\
\hline$\Delta$ LVEDP $(n$ & $2 \pm 1.8^{*}$ & $1.9 \pm 1.5^{\star}$ & $2.2 \pm 1.3^{*}$ & $1.9 \pm 2.3$ & $2.1 \pm 1.5^{\star}$ & $2.1 \pm 1.7$ \\
\hline$\Delta$ LVEDV $(\%)$ & $18 \pm 16.8^{*}$ & $19.8 \pm 15.4^{\star}$ & $16.8 \pm 14.8^{*}$ & $16.6 \pm 17.1^{\star}$ & $14.8 \pm 13.9 *$ & $14.4 \pm 23.1$ \\
\hline$\Delta \mathrm{LV} \mathrm{dP/dt_{ \operatorname {max } }}$ & $1.9 \pm 5$ & $4.3 \pm 6.2$ & $3.2 \pm 7.4$ & $1.4 \pm 5.6$ & $5.5 \pm 6.4$ & $8.5 \pm 10.9$ \\
\hline$\Delta$ RVESP (\%) & $9.8 \pm 2.8^{*}$ & $10.9 \pm 3.2^{\star}$ & $10.7 \pm 4.6^{\star}$ & $12.2 \pm 8^{*}$ & $13.6 \pm 9.9 *$ & $10.6 \pm 4.6^{*}$ \\
\hline$\Delta$ RVEDP $(\mathrm{mmHg})$ & $1.4 \pm 1 *$ & $1.5 \pm 1.9$ & $1.5 \pm 0.9 *$ & $1 \pm 1.5$ & $0.8 \pm 1$ & $1.1 \pm 1.3$ \\
\hline$\Delta \mathrm{RV} \mathrm{dP} / \mathrm{dt}_{\max }(\%)$ & $-1.5 \pm 9.4$ & $-6.2 \pm 16.3$ & $1.4 \pm 8.5$ & $0.8 \pm 4.5$ & $-4.9 \pm 7.7$ & $5 \pm 11.9$ \\
\hline$\Delta \mathrm{LAP}_{\max }(\mathrm{mmHg})$ & $-0.2 \pm 4.2$ & $-1.4 \pm 4.3$ & $-0.8 \pm 4.7$ & $1.2 \pm 3 *$ & $2.4 \pm 2.5$ & $-2.9 \pm 6.5$ \\
\hline$\Delta \mathrm{RAP}_{\max }(\mathrm{mmHg})$ & $-2.5 \pm 3.2$ & $-4 \pm 2.7 \star \S$ & $-2.3 \pm 3^{\#}$ & $0.2 \pm 3.2$ & $-0.1 \pm 3^{\wedge}$ & $-3.5 \pm 4.6$ \\
\hline$\Delta$ Forward flow ( $r$ & $1.6 \pm 3.3$ & $2.4 \pm 3.6$ & $-0.3 \pm 4.9$ & $0.8 \pm 4$ & $1.8 \pm 4.1$ & $3.2 \pm 6.4$ \\
\hline$\Delta$ Diastolic MR $(\mathrm{mL})$ & $-1.8 \pm 2.4$ & $-2 \pm 2.5$ & $-2.9 \pm 4$ & $-3.5 \pm 2.7 \star$ & $-3.5 \pm 2.4^{\star \wedge}$ & $-2.9 \pm 3.4$ \\
\hline
\end{tabular}

Presented are mean \pm SEM. Abbreviations as in table 1. * indicates $P<0.05$ compared to baseline, ${ }^{\wedge}$ indicates $P<0.05$ compared to atrial pacing for same pace site, $\S$ indicates $P<0.05$ compared to BiV pacing for same atrial mode, \# indicates $\mathrm{P}<0.05$ compared to LV pacing for the same atrial mode. $\mathrm{N}=7$ animals.

Table 3. Timing and intervals of intracardiac events at the optimal AV-delay.

\begin{tabular}{lcccccc} 
& \multicolumn{3}{c}{ Atrial pacing } & \multicolumn{3}{c}{ Atrial sensing } \\
& BiV & LV & RV & BiV & LV & RV \\
P-wave duration (ms) & $116 \pm 3$ & $114 \pm 5$ & $113 \pm 5$ & $77 \pm 7^{\star}$ & $76 \pm 7^{\star}$ & $75 \pm 4^{\star}$ \\
\hline IAD (ms) & $29 \pm 5$ & $30 \pm 5$ & $26 \pm 6$ & $20 \pm 5$ & $14 \pm 5$ & $18 \pm 6^{\star}$ \\
\hline QRS duration (ms) & $86 \pm 5$ & $109 \pm 4^{\wedge}$ & $85 \pm 5^{\S}$ & $83 \pm 5$ & $111 \pm 5^{\wedge}$ & $85 \pm 4^{\S}$ \\
\hline IVD (ms) & $-4 \pm 5$ & $19 \pm 5^{\wedge}$ & $-9 \pm 6^{\S}$ & $-5 \pm 3$ & $13 \pm 2^{\wedge}$ & $-16 \pm 4^{\wedge}$ \\
\hline Right-eAVD (ms) & $166 \pm 7$ & $187 \pm 11$ & $164 \pm 13$ & $212 \pm 29$ & $228 \pm 28$ & $216 \pm 26$ \\
\hline Left-eAVD (ms) & $140 \pm 8$ & $142 \pm 16$ & $147 \pm 11$ & $198 \pm 31$ & $201 \pm 28$ & $214 \pm 24^{\star}$ \\
\hline (might-left-eAVD (ms) & $26 \pm 7$ & $45 \pm 8^{\wedge}$ & $17 \pm 5 \S$ & $15 \pm 3$ & $27 \pm 4^{\wedge}$ & $2 \pm 4^{\star}$ \\
\hline Mean-eAVD (ms) & $153 \pm 7$ & $165 \pm 13$ & $156 \pm 12$ & $205 \pm 30$ & $214 \pm 28$ & $215 \pm 25^{\star}$ \\
\hline Mean-eAVD-HR (\%) & $26 \pm 1$ & $27 \pm 2$ & $26 \pm 2$ & $28 \pm 4$ & $30 \pm 4$ & $31 \pm 3$ \\
\hline Presen
\end{tabular}

Presented are mean $\pm S E M$. BiV: biventricular, LV: left ventricle, RV: right ventricle, IAD: interatrial delay, IVD: interventricular dyssynchrony, eAVD: effective AV-delay, HR: heart rate. * indicates $P<0.05$ compared to atrial pacing, ${ }^{\wedge}$ indicates $\mathrm{P}<0.05$ compared to BiV pacing for same atrial mode, $\$$ indicates $\mathrm{P}<0.05$ compared to LV pacing for same atrial mode. $\mathrm{N}=7$ animals. 


\section{Differences in timing of cardiac events during various pacing modes}

The timings and intervals of various parameters at $A V_{\text {opt }}$ are presented in table 3. P-wave duration was significantly shorter in A-S compared to A-P during BiV, LV and RV pacing (75-77ms vs. 113-116ms, respectively). This indicates a prolonged total activation time of the atria and larger interatrial dyssynchrony, which is supported by a longer interval between RA dP/dt $t_{\max }$ and LA dP/dt max $_{\text {in }}$ A-P than in A-S mode (ranging from 14-20ms in A-S compared to 26-30ms in A-P). Right-eAVD, left-eAVD and mean-eAVD were larger during A-S, however only significant for left-eAVD (214 $\pm 24 \mathrm{~ms}$ vs $147 \pm 11 \mathrm{~ms})$ and meaneAVD ( $215 \pm 25 \mathrm{~ms}$ vs $156 \pm 12 \mathrm{~ms}$ ) during RV pacing (see table 3 ). Where D right-left-eAVD became as large as $45 \mathrm{~ms}$ for LV pacing in A-P mode, it was shorter in A-S, however only significantly for RV pacing ( $2 \pm 4 \mathrm{~ms})$.

In A-P mode the interventricular dyssynchrony (interval between times of LV and RV dP/ $\left.\mathrm{dt}_{\text {max }}\right)$ was significantly different between LV pacing $(19 \pm 5 \mathrm{~ms})$ and BiV $(-4 \pm 5 \mathrm{~ms})$ or RV pacing $(-9 \pm 6 \mathrm{~ms}$, table 3$)$ and QRS duration was significantly longer during LV pacing $(109 \pm 4 \mathrm{~ms})$ than during BiV $(86 \pm 5 \mathrm{~ms})$ and RV pacing $(85 \pm 5 \mathrm{~ms})$. Comparable results were observed in A-S mode. The interval between LV and RV dP/dt $t_{\max }$ differed between all three different ventricular pacing sites (see table 3).

\section{Optimization of AV-delay using effective mechanical AV-delay}

Figure 6 shows the average AV-delay optimization curves based on cardiac output for six pacing protocols. When cardiac output (\% of maximal change) was plotted against the absolute programmed AV-delay, the optimal AV-delay was shorter for the A-S than for the A-P curves. The opposite was true when cardiac output change was plotted against righteAVD and left-eAVD (middle and right upper panels of figure 6). Mean-eAVD showed less difference between the six optimization plots and the optima were even closer together when mean-eAVD was expressed as a percentage of RR-interval. For this RR-normalized mean-eAVD the curves of the different pacing modes fell well on top of each other with a common optimum with a mean-eAVD of $\sim 25 \%$ of the RR-interval. Stroke work and mean arterial pressure showed comparable patterns when plotted against the various effective AV-delays (data not shown).

Mean optimal AV-delays calculated from all 39 pacing settings in all seven experiments are presented in table $\mathbf{4}$ for cardiac output, stroke work and mean arterial pressure. For all three hemodynamic parameters optimal right-eAVD, left-eAVD and mean-eAVD were significantly longer compared to the optimal programmed AV-delay. The coefficient of variance for the optimal AV-delays of the cardiac output and stroke work curves was lowest for mean-eAVD corrected for HR. 


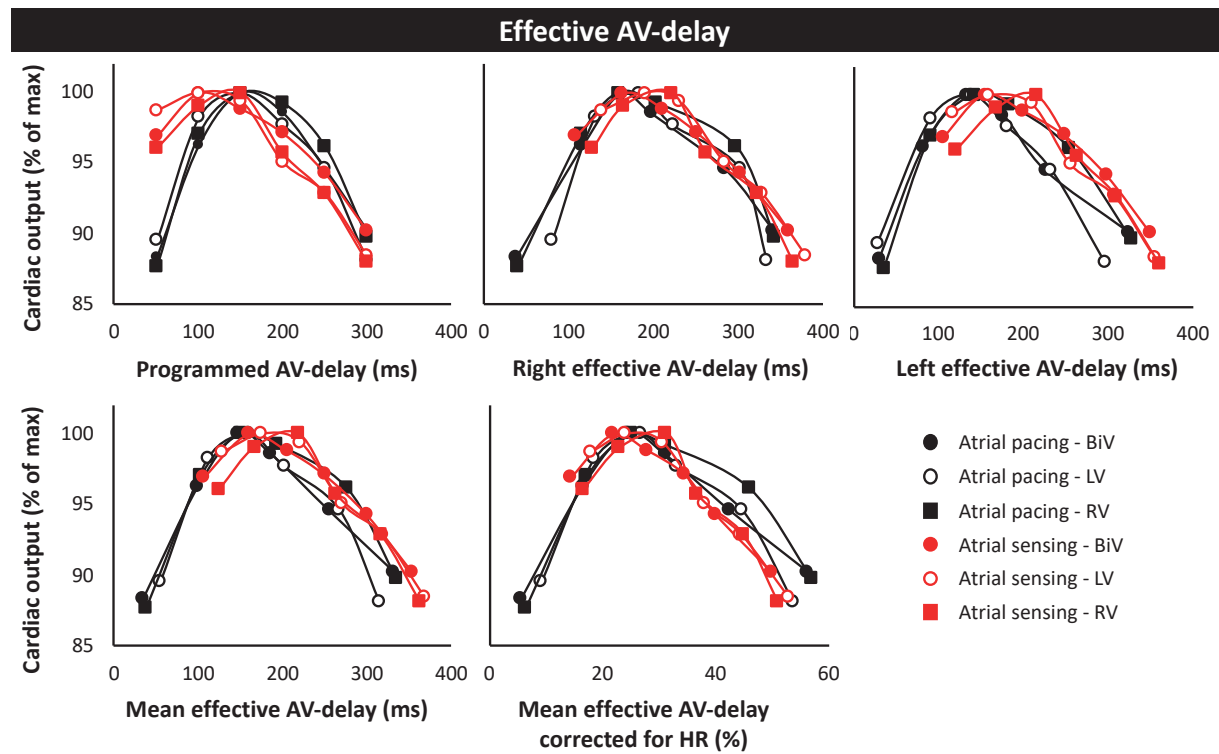

Figure 6. Average AV-optimization curves for cardiac output presented as percentage of maximal value for all six pacing modes. The $x$-axis changes for every panel. The paced AV-delay (upper left), right effective AV-delay (upper middle), left effective AV-delay (upper right), mean effective AV-delay (lower left) and mean effective AV-delay as percentage of RR-interval (lower right) are being presented. Right-eAVD and left-eAVD is the time difference between RA dP/dt max $_{\text {and }}$ an $\mathrm{dP} / \mathrm{dt}_{\max }$ and LA dP/ $\mathrm{dtmax}$ and $\mathrm{LV} \mathrm{dP} / \mathrm{dt}_{\max }$ respectively. The mean-eAVD is the average between right and left-eAVD.

Table 4. Values for optimal programmed and effective AV-delays and their coefficient of variation.

\begin{tabular}{cccccccc} 
& & & $\begin{array}{c}\text { Programmed } \\
\text { AV-delay (ms) }\end{array}$ & $\begin{array}{c}\text { Right-eAVD } \\
(\mathbf{m s})\end{array}$ & $\begin{array}{c}\text { Left-eAVD } \\
(\mathbf{m s})\end{array}$ & $\begin{array}{c}\text { Mean-eAVD } \\
(\mathbf{m s})\end{array}$ & $\begin{array}{c}\text { Mean-eAVD } \\
\text { corrected for } \\
\text { HR }(\%)\end{array}$ \\
\multirow{2}{*}{ Co } & Mean \pm SEM & $149 \pm 47$ & $190 \pm 54^{\star}$ & $166 \pm 58^{\star}$ & $178 \pm 55^{\star}$ & $27 \pm 7$ \\
\cline { 2 - 7 } & $\mathbf{C V}$ & 0.31 & 0.28 & 0.35 & 0.31 & 0.27 \\
\hline \multirow{2}{*}{ SW } & Mean \pm SEM & $138 \pm 56$ & $178 \pm 53^{\star}$ & $157 \pm 56^{\star}$ & $168 \pm 53^{\star}$ & $25 \pm 7$ \\
\cline { 2 - 7 } & $\mathbf{C V}$ & 0.40 & 0.30 & 0.35 & 0.32 & 0.27 \\
\hline \multirow{2}{*}{ MAP } & Mean \pm SEM & $154 \pm 60$ & $194 \pm 53^{\star}$ & $172 \pm 57^{\star}$ & $183 \pm 54^{\star}$ & $28 \pm 8$ \\
\cline { 2 - 7 } & $\mathbf{C V}$ & 0.39 & 0.27 & 0.33 & 0.30 & 0.28 \\
\hline
\end{tabular}

$\overline{\text { Values were calculated from all individual data. eAVD: effective atrioventricular delay, HR: heart rate, }}$ CO: cardiac output, SW: stroke work, MAP: mean arterial pressure, CV: coefficient of variation. * indicates $\mathrm{P}<0.05$ compared to programmed AV-delay.

\section{DISCUSSION}

The main finding of this animal study is that the AV-delay that creates the maximal increase in cardiac output ("optimal AV-delay"), is influenced by altered diastolic filling patterns due to changes in interatrial delay (atrial pacing vs. atrial sensing) and interventricular 
dyssynchrony (various ventricular pacing sites). Calculation of the mean effective AVdelay, normalized for heart rate, can estimate the optimal AV-delay in a manner that is relatively independent of IAD or ventricular pacing site and might therefore be an interesting parameter for future pacemaker optimization.

\section{The influence of interatrial delay on the optimal AV-delay}

The difference between atrial pacing and atrial sensing in 2-chamber pacemakers has been studied since the late 1980s. Wish et al showed that invasively measured stroke volume decreased when pacing mode was changed from DVI (atrial pacing) to VDD (atrial sensing) without adapting the programmed AV-delay. They suggested that this change was the result of an increase in LA to LV depolarization time due to a decrease in IAD [11]. Comparable results were seen in a study by Janosik et al in which Doppler-derived cardiac output was used to examine the optimal AV-delay during DVI and VDD pacing [10]. Kyriacou et al have previously shown that IAD is longer during A-P mode and that the programmed AV-delay should therefore be longer compared to A-S to prevent truncation of ventricular filling in CRT patients [12]. Along with these previous studies, we found that there was a tendency for optimal AV-delay to be shorter during A-S compared to A-P. One of the possible reasons for the small difference in optimal AV-delay between A-S and A-P was that the optimal AV-delay was based on only 5 different AV-delays with a relatively large interval of $50 \mathrm{~ms}$, while the optimal AV-delay could well be somewhere between two AV-delays. Using second degree polynomial expression the optimal AV-delay could be calculated more accurately, leading to greater differences between A-P and A-S mode ( 40$50 \mathrm{~ms}$ ). In this study the maximal increases in cardiac output, stroke work and mean arterial pressure were similar for A-S and A-P, indicating that similar hemodynamic conditions can be achieved by both pacing modes as long as AV-delay is properly optimized.

Like in other studies, the time difference between RA and LA activation was longer during A-P compared to A-S, evidenced by an increased P-wave width on the ECG and a larger IAD (difference between time of RA and LA dP/dt ${ }_{\text {max }}$ ). This can be explained by delayed activation of the LA because the pacing electrode is generally positioned in the RA appendage, more remote from the $L A$, and besides that electrical conduction does not follow the Bachman's bundle. These findings are in line with a clinical study by Dabrowska et al in which P-wave duration was shown to correlate with atrial electromechanical delay measured using tissue Doppler during different atrial pacing modalities [13].

In our study, the P-wave width increased by $\sim 40 \mathrm{~ms}$ when pacing mode was switched from A-S to A-P, while the IAD only increased by $10-15 \mathrm{~ms}$. This relatively small increase in IAD may be explained by the fact that $P$-wave width is the difference between the very earliest and latest activated atrial tissue, whereas IAD is determined by the mechanical activity of large parts of both atria. The 10-15ms values of IAD in our study are smaller than in human studies, in which a delay in left atrial contraction of $\sim 60 \mathrm{~ms}$ was found when comparing A-P to A-S $[12,14]$. Possible explanations might be species differences and the different 
determination of IAD. Our study used atrial pressure rise times, while previous studies used the moment of atrial contraction onset, derived from tissue Doppler imaging or electrical mapping in the coronary sinus.

During A-S mode left- and right-eAVD were longer than during A-P mode for every AVdelay. This can be explained by the fact that atrial activation and possibly contraction had already started before atrial activity was sensed by the pacemaker in A-S mode. The interval between the atrial pace spike and atrial $\mathrm{dP} / \mathrm{dt}_{\max }$ was $\sim 100 \mathrm{~ms}$ in A-P mode, while the time between the moment of atrial sensing and atrial $\mathrm{dP} / \mathrm{dt}_{\max }$ in A-S mode was only $\sim 30 \mathrm{~ms}$. When keeping the paced AV-delay stable the effective AV-delay will consequently be longer because of the earlier activation of the atria.

These differences between the A-S and A-P mode were clearly visible in the LV diastolic filling patterns. Since left-eAVD was longer during A-S, the time between the mitral E and A-wave was longer for every corresponding AV-delay than during A-P. This resulted in the absence of A-wave truncation at the shortest AV-delay, preventing a decrease in forward flow due to suboptimal ventricular filling. Furthermore, E and A-wave fusion happened at a shorter AV-delay in A-S compared to the A-P mode, supporting the idea that a shorter AV-delay should be programmed when using VDD pacemaker therapy.

The increase in IAD when switching from A-S to A-P was larger during LV pacing $(16 \mathrm{~ms})$ than during BiV (7-9ms) and RV (7-8ms). A possible explanation might be that during LV pacing at short interval the early LV contraction and pressure development also increases - through upward motion of the mitral valve leaflets - atrial pressure development and thereby shift the moment of atrial $\mathrm{dP} / \mathrm{dt}_{\text {max }}$. Interestingly, also IVD seems to differ between A-S and A-P mode, especially during LV (from 19 to 13ms) and RV (-9 to -16ms). This could be a result of changes in ventricular filling due to prolonged atrial contraction in A-P mode, causing ventricular $\mathrm{dP} / \mathrm{dt}_{\max }$ to occur at a slightly different moment.

The increase in optimal AV-delay between A-S and A-P was comparable to the increase in IAD during BiV and RV pacing. However, for LV pacing the increase in optimal AV-delay (29ms) was larger than the increase in IAD (16ms). The latter might be also explained by the greater IVD during LV pacing compared to RV and BiV pacing. Other clinical studies found correlations between the IAD and the optimal AV-delay [11, 12, 14], though the ratio was not 1:1 in all cases, suggesting that there are other factors besides IAD that influence the optimal AV-delay.

\section{The influence of interventricular dyssynchrony (pacing site) on optimal AV-delay}

To our knowledge this is the first (experimental) study indicating that the optimal AVdelay is influenced by ventricular pacing site. This evidence comes predominantly from the measurements during LV pacing. 
Pacing induced pre-excitation of the LV leads to a significantly shorter LA-LV interval compared to RA-RV interval. Also the LA-LV interval is shorter during LV pacing than during RV or BiV pacing. In other words, atrial pacing leads to a delayed activation of the LA compared to the RA (RA pre-excitation), while LV pacing (LV pre-excitation) shortens the left effective AV-delay compared to the right heart. In our study, it leads to a longer optimal programmed AV-delay during LV pacing in A-P mode. Similar results might be present during RV pacing, however RV apex pacing did not lead to significant amounts of interventricular dyssynchrony in our porcine study.

It is important to realize that the present study has been performed in pigs after creation of complete AV-block, eliminating the possibility of fusion of the pacing-induced and intrinsic activation. Such fusion pacing is frequently employed, because it may lead to even better resynchronization than BiV pacing [15-17].

\section{Mean effective AV-delay as predictor for optimal hemodynamic response}

The results discussed in the previous paragraphs indicate that it is important to take both atrial pacing mode and ventricular pacing site into account when defining the optimal AVdelay for an individual. From this perspective we calculated the ventricle-specific effective AV-delay. However, in previous animal studies and computer simulations about optimizing interventricular delays we have shown that optimal LV function was reached using LV pre-excitation while RV function was better during RV pre-excitation and that the best overall cardiac output was achieved using a setting in between the optimal settings for the LV and RV [7] (see also chapter 6). Along the same lines, we hypothesized that a mean effective AV-delay would provide an even better estimation of the optimal AV-delay in a given setting. This idea has meanwhile been supported by simulations in the CircAdapt computer model [18]

One important implication of these results is that, while most research focusses on leftsided AV-optimization, this study shows that filling of both ventricles should be taken into account. Commonly the golden standard in AV-optimization uses echocardiographyderived mitral flow patterns. The optimal AV-delay is considered the shortest AV-delay with A-wave truncation in the absence of $E$ and $A$-wave fusion [19]. This technique focusses on left-sided optimization and this might be one of the reasons why long-term benefits of AVoptimization have not been observed in clinical trials, and consequently, not implemented in the guidelines. Other optimization algorithms use electrograms [20] or accelerometer signals [21], but it is unclear how these relate to the mean effective AV-delay.

The mean effective AV-delay concept was further finetuned by normalizing AV-delay for RR-interval. This idea was based on several patient studies. Rafie et al have shown that the optimal AV-delay decreased when patients receiving CRT were paced at a higher frequency. Pacing at this shorter optimal AV-delay improved diastolic filling time, mitral inflow VTI, systolic ejection time and NYHA class compared to a fixed AV-delay [22]. These findings 
were replicated by Kyriacou et al in CRT patients in atrial sense mode in rest and during exercise and in atrial pace mode at different pacing rates [12]. Furthermore, computer simulations from our group have shown that the mean-eAVD corrected for RR shows less variability than absolute mean effective AV-delay when simulations were performed at different pacing rates [18]. The mean-eAVD as calculated in pigs was $\sim 50 \mathrm{~ms}$ longer in A-S mode compared to A-P for any ventricular pacing side, while this difference nearly completely disappeared when correcting it for heart rate. This provides further evidence for the influence of the heart rate on the optimal AV-delay.

\section{Clinical perspective and future directions}

The mean effective AV-delay is a global, bilateral measure of AV-coupling and is a better parameter for determining the optimal AV-delay in a wider variety of pacing settings. To implement the mean effective AV-delay concept clinically, detailed information on activation of RA, LA, RV and LV is necessary. Electrical RA-RV intervals can be derived from the pacemaker (sensed or paced RA-RV interval). P-wave width may be, and sometimes is, used to define IAD. Interventricular dyssynchrony can be derived from the QRS complex in case of clear dyssynchrony or from interlead conduction times [23-26]. A combination of these electrical parameters might subsequently be used to calculate mean effective AV-delay.

For deriving mean effective AV-delay from mechanical information, echocardiography can be used to define right and left sided AV-delay by examining tricuspid and mitral filling patterns. While a single measurement of mean effective AV-delay in rest is possible using the abovementioned electrical and mechanical information, ambulatory repeated measurements can so far only be performed using the electrical information. Implanted accelerometers may be used to assess interventricular dyssynchrony by analyzing splitting of the second heart sound (chapter 5, [27]), but further studies are needed to derive also atrial information from these sensors.

Automatic optimization algorithms based on electrical signals have already been implemented in commercially available pacemakers. However, randomized clinical trials did not always show significant improvements compared to echocardiographic optimization or default settings $[20,28,29]$, possibly because they focus on left-sided optimization of AV-delay. The CLEAR and RESPOND trials comparing the mechanical SonR accelerometerbased optimization to default settings in CRT patients indeed showed comparable clinical outcome in both groups [21,30], suggesting that such mechanical sensors may be at least as useful as echocardiographic measures. It would be even more interesting to explore the use of an algorithm to automatically define the optimal mean effective AV-delay based on a combination of pacing lead electrograms and accelerometer signals. 


\section{Limitations}

This study concerns experiments in a small number of porcine hearts. While this species is frequently used for cardiovascular research, a limitation is that the degree of dyssynchrony induced by LV and RV pacing is smaller than in other species like man and dog. We hypothesize that the concept of mean effective AV-delay works better in humans, due to a greater amount of interventricular dyssynchrony, which was also the case in the CircAdapt computer model [18].

A model of total AV-block was used because this allowed observing the isolated effect of changes in AV-delay, while in many patients AV-conduction might be present. In such situation fusion of intrinsic conduction and paced activation waves may occur, thereby changing the amount of interventricular dyssynchrony.

Furthermore, the optimal AV-delay was based on acute hemodynamic improvements while an autonomic regulatory response characterized by changes in peripheral arterial resistance and pressure might be likely to occur. Beat-to-beat measurements in patients have shown though, that the initial increment in cardiac output would sustain throughout a longer period [31], but acute hemodynamic improvements may not (fully) translate into clinical improvements.

\section{CONCLUSION}

This experimental animal study has identified interatrial delay and interventricular dyssynchrony as important influencers for the optimal AV-delay to be programmed. The optimal AV-delay increases with greater interatrial delay due to RA pre-excitation and with pre-excitation of the LV. Furthermore, the mean effective AV-delay corrected for heart rate seems a good and widely applicable predictor for the optimal hemodynamic effect of pacing since it takes into account both RV and LV function. Additional research should be performed to examine how this parameter can be implemented to optimize atrioventricular optimization in patients. 


\section{REFERENCES}

1 Houthuizen P, Bracke FA, van Gelder BM. Atrioventricular and interventricular delay optimization in cardiac resynchronization therapy: physiological principles and overview of available methods. Heart Fail Rev. 2011;16(3):263-76.

2 Auricchio A, Stellbrink C, Sack S, Block M, Vogt J, Bakker P, et al. The Pacing Therapies for Congestive Heart Failure (PATH-CHF) study: rationale, design, and endpoints of a prospective randomized multicenter study. Am J Cardiol. 1999;83(5B):130D-5D.

3 Bogaard MD, Kirkels JH, Hauer RN, Loh P, Doevendans PA, Meine M. Should we optimize cardiac resynchronization therapy during exercise? J Cardiovasc Electrophysiol. 2010;21(11):1307-16.

4 Zweerink A, Salden OAE, van Everdingen WM, de Roest GJ, van de Ven PM, Cramer MJ, et al. Hemodynamic Optimization in Cardiac Resynchronization Therapy: Should We Aim for dP/ dtmax or Stroke Work? JACC Clin Electrophysiol. 2019;5(9):1013-25.

5 Whinnett ZI, Davies JE, Willson K, Manisty CH, Chow AW, Foale RA, et al. Haemodynamic effects of changes in atrioventricular and interventricular delay in cardiac resynchronisation therapy show a consistent pattern: analysis of shape, magnitude and relative importance of atrioventricular and interventricular delay. Heart. 2006;92(11):1628-34.

6 Antonini L, Auriti A, Pasceri V, Meo A, Pristipino C, Varveri A, et al. Optimization of the atrioventricular delay in sequential and biventricular pacing: physiological bases, critical review, and new purposes. Europace. 2012;14(7):929-38.

7 Willemen E, Schreurs R, Huntjens PR, Strik M, Plank G, Vigmond E, et al. The Left and Right Ventricles Respond Differently to Variation of Pacing Delays in Cardiac Resynchronization Therapy: A Combined Experimental- Computational Approach. Front Physiol. 2019;10:17.

8 Cha YM, Nishimura RA, Hayes DL. Difference in mechanical atrioventricular delay between atrial sensing and atrial pacing modes in patients with hypertrophic and dilated cardiomyopathy: an electrical hemodynamic catheterization study. J Interv Card Electrophysiol. 2002;6(2):133-40.

9 Di Pede F, Gasparini G, De Piccoli B, Yu Y, Cuesta F, Raviele A. Hemodynamic effects of atrial septal pacing in cardiac resynchronization therapy patients. J Cardiovasc Electrophysiol. 2005;16(12):1273-8.

10 Janosik DL, Pearson AC, Buckingham TA, Labovitz AJ, Redd RM, Mrosek D. The hemodynamic benefit of differential atrioventricular delay intervals for sensed and paced atrial events during physiologic pacing. J Am Coll Cardiol. 1989;14(2):499-507.

11 Wish M, Fletcher RD, Gottdiener JS, Cohen Al. Importance of left atrial timing in the programming of dual-chamber pacemakers. Am J Cardiol. 1987;60(7):566-71.

12 Kyriacou A, Rajkumar CA, Pabari PA, Sohaib SMA, Willson K, Peters NS, et al. Distinct impacts of heart rate and right atrial-pacing on left atrial mechanical activation and optimal AV delay in CRT. Pacing Clin Electrophysiol. 2018.

13 Dabrowska-Kugacka A, Lewicka-Nowak E, Rucinski P, Zagozdzon P, Raczak G, Kutarski A. Relationship between P-wave duration and atrial electromechanical delay assessed by tissue Doppler echocardiography. Pacing Clin Electrophysiol. 2011;34(1):23-31.

14 Levin V, Razavi M, Coll R, Coles JA, Jr., Sambelashvili A. Interatrial conduction correlates with optimal atrioventricular timing in cardiac resynchronization therapy devices. Pacing Clin Electrophysiol. 2011;34(4):443-9.

15 Strik M, van Middendorp LB, Houthuizen P, Ploux S, van Hunnik A, Kuiper M, et al. Interplay of electrical wavefronts as determinant of the response to cardiac resynchronization therapy in dyssynchronous canine hearts. Circ Arrhythm Electrophysiol. 2013;6(5):924-31.

16 AlTurki A, Lima PY, Bernier ML, Garcia D, Vidal A, Toscani B, et al. Optimization of Chronic Cardiac Resynchronization Therapy Using Fusion Pacing Algorithm Improves Echocardiographic Response. CJC Open. 2020;2(2):62-70.

17 Ter Horst IAH, Bogaard MD, Tuinenburg AE, Mast TP, de Boer TP, Doevendans P, et al. The concept of triple wavefront fusion during biventricular pacing: Using the EGM to produce the best acute hemodynamic improvement in CRT. Pacing Clin Electrophysiol. 2017;40(7):873-82. 
18 Willemen E, Huntjens PR, Delhaas T, Lumens J, Prinzen FW. Importance of effective atrioventricular delay for ventricular filling and cardiac pump function. 2020.

19 International Working Group on Quantitative O, Sohaib SM, Whinnett ZI, Ellenbogen KA, Stellbrink C, Quinn TA, et al. Cardiac resynchronisation therapy optimisation strategies: systematic classification, detailed analysis, minimum standards and a roadmap for development and testing. Int J Cardiol. 2013;170(2):118-31.

20 Ellenbogen KA, Gold MR, Meyer TE, Fernndez Lozano I, Mittal S, Waggoner AD, et al. Primary results from the SmartDelay determined AV optimization: a comparison to other AV delay methods used in cardiac resynchronization therapy (SMART-AV) trial: a randomized trial comparing empirical, echocardiography-guided, and algorithmic atrioventricular delay programming in cardiac resynchronization therapy. Circulation. 2010;122(25):2660-8.

21 Brugada J, Delnoy PP, Brachmann J, Reynolds D, Padeletti L, Noelker G, et al. Contractility sensor-guided optimization of cardiac resynchronization therapy: results from the RESPONDCRT trial. Eur Heart J. 2016.

22 Rafie R, Qamruddin S, Ozhand A, Taha N, Naqvi TZ. Shortening of atrioventricular delay at increased atrial paced heart rates improves diastolic filling and functional class in patients with biventricular pacing. Cardiovasc Ultrasound. 2012;10:2.

23 Sassone B, Gabrieli L, Sacca S, Boggian G, Fusco A, Pratola C, et al. Value of right ventricular-left ventricular interlead electrical delay to predict reverse remodelling in cardiac resynchronization therapy: the INTER-V pilot study. Europace. 2010;12(1):78-83.

24 Sommer A, Kronborg M, Gerder C, Kristensen J, Nielsen J. Ventricular paced interlead electrical delay and response to cardiac resynchronization therapy. Europace. 2018;20:i53.

25 Stabile G, D'Onofrio A, Pepi P, De Simone A, Santamaria M, Caico SI, et al. Interlead anatomic and electrical distance predict outcome in CRT patients. Heart Rhythm. 2015;12(11):2221-9.

26 Tahri JB, Hansen TF, Storkas HS, Lauridsen TK, Olsen FJ, Iversen A, et al. Interlead electrical delays and scar tissue: Response to cardiac resynchronization therapy in patients with ischemic cardiomyopathy. Pacing Clin Electrophysiol. 2019;42(5):530-6.

27 Luo H, Westphal P, Shahmohammadi M, Heckman LIB, Kuiper M, Cornelussen RN, et al. Second heart sound splitting as an indicator of interventricular mechanical dyssynchrony using a novel splitting detection algorithm. Physiol Rep. 2021;9(1):e14687.

28 Krum H, Lemke B, Birnie D, Lee KL, Aonuma K, Starling RC, et al. A novel algorithm for individualized cardiac resynchronization therapy: rationale and design of the adaptive cardiac resynchronization therapy trial. Am Heart J. 2012;163(5):747-52 e1.

29 Thibault B, Ritter P, Bode K, Calo L, Mondesert B, Mangual JO, et al. Dynamic programming of atrioventricular delay improves electrical synchrony in a multicenter cardiac resynchronization therapy study. Heart Rhythm. 2019;16(7):1047-56.

30 Delnoy PP, Ritter P, Naegele H, Orazi S, Szwed H, Zupan I, et al. Association between frequent cardiac resynchronization therapy optimization and long-term clinical response: a post hoc analysis of the Clinical Evaluation on Advanced Resynchronization (CLEAR) pilot study. Europace. 2013;15(8):1174-81.

31 Manisty CH, Al-Hussaini A, Unsworth B, Baruah R, Pabari PA, Mayet J, et al. The acute effects of changes to AV delay on BP and stroke volume: potential implications for design of pacemaker optimization protocols. Circ Arrhythm Electrophysiol. 2012;5(1):122-30. 




\section{ABSTRACT}

\section{Introduction}

The SonR system is an algorithm that uses data from an accelerometer in the right atrial (RA) or right ventricular (RV) pacing lead that is used to automatically optimize atrioventricular (AV) and interventricular [1] delay in patients receiving cardiac resynchronization therapy. This animal study aims to investigate the influence of cardiac contractility, atrioventricular coupling and interventricular dyssynchrony on the amplitude of the first heart sound (SonR1), in order to better understand and possibly improve the algorithm.

\section{Methods and results}

Studies were performed in 9 anesthetized dogs with left bundle branch block or total atrioventricular (AV) block with right ventricular (RV) pacing, using tachypacing or isoflurane to induce heart failure. Invasive left (LV) and RV pressures and RA and RV SonR1 signals were measured during biventricular [2] and LV pacing at various AV-delays and compared to baseline values of intrinsic conduction. The animals were divided in a non-responding (NResp, $\mathrm{N}=4$ ) and responding (Resp, $\mathrm{N}=5$ ) group based on a $>10 \%$ increase of $\mathrm{LV} \mathrm{dP/dt_{ \operatorname {max } }}$ during LV or BiV pacing. Dobutamine induced improvement of cardiac contractility in 3 animals revealed strong correlations between LV and RV dP/dt max and RA and RV SonR1. During BiV pacing at various AV-delays no consistent changes in LV pressures, LV and RV $\mathrm{dP} / \mathrm{dt}_{\text {max }}$ mechanical interventricular dyssynchrony (MIVD) and SonR1 amplitudes were found in NResp animals. in Resp animals BiV and LV pacing increased systolic LV pressure and $L V d P / d t_{\text {max }}$ at the optimal AV-delay $\left(\mathrm{AV}_{\text {opt }}\right)$ compared to baseline $(B L)$ values, along with an increase in RA (and to a lesser extent RV) SonR1. During pacing at short AV delays SonR1 values were at least as high as during $A V_{\text {opt' }}$ despite lower hemodynamic benefit, indicating an effect of atrio-ventricular interaction on SonR1. Moreover, LV pacing at $\mathrm{AV}_{\text {short }}$ revealed similar SonR1 values as during BiV pacing, although MIVD was significantly larger during LV pacing.

\section{Conclusions}

The SonR1 amplitude is influenced by changes in cardiac contractility and time interval between atrial and ventricular contraction, while interventricular dyssynchrony plays a minor role. Placement of the SonR sensor in the RA seems to assess cardiac function better than an RV-placed sensor. 


\section{INTRODUCTION}

Cardiac resynchronization therapy (CRT) is a well-established therapy for treatment of patients with heart failure and conduction abnormalities, coinciding with a wide QRS complex, especially left bundle branch block (LBBB) [3]. CRT employs (almost) simultaneous stimulation of the right (RV) and left ventricle (LV), usually with a certain delay with respect to the sensed or paced atrial activity. A large number of hemodynamic studies (reviewed in [4]) have shown that optimization of the delays between atrial and ventricular stimulation (AV-delay) and between RV and LV stimulation (VV-delay) leads to the best hemodynamic improvement. However, such echocardiographic optimization is performed in a recumbent, resting position, whereas patients may especially need the optimal stimulation setting when exercising. Moreover, the optimal delays may not be constant over time.

To this purpose, the SonR system has been introduced, which contains a sensor embedded in the right ventricular (RV) or right atrial (RA) pacing lead to record and amplify myocardial vibrations during the entire cardiac cycle. Until now, most attention has been paid to the vibrations, associated with the first heart sound (S1) and is referred to as peak endocardial acceleration (PEA) or, more recently, SonR1.

Although the exact origin of the heart sounds are not entirely clear, currently the most accepted mechanism is the one introduced by Rushmer [5]. He suggested that the acceleration and deceleration of the blood in the 'cardiohemic' system induces the heart sound vibrations. These velocity changes could be abrupt cessation of backflow of blood in the atrial and ventricular cavities, creating vibrations in the valve, the column of blood and myocardial structures surrounding it. Along the lines of this hypothesis, more forceful contractions, as during increased contractility dobutamine stress testing, showed good correlations between SonR1 recorded in the RV or right atrium (RA) and LV and RV dP/ $\mathrm{dt}_{\max }[6-8]$ in healthy pigs and sheep.

However, more complex behavior of SonR1 may be expected during CRT. Firstly, CRT aims at increasing contractility by reducing atrioventricular and interventricular asynchrony, which may lead to a larger S1 amplitude. Moreover, the S1 amplitude is affected by the interplay between ventricular and atrial pressures. S1 is smaller at long AV-delays, when the mitral valve is able to close gently at the moment of reversal of the transmitral AV pressure gradient. In contrast, at shorter AV-delays, end-diastolic filling flow is abruptly interrupted by ventricular contraction onset at a time when left atrial (LA) pressure is still high, generating high amplitude vibrations resulting in a louder S1 [9]. Since the S1 and the SonR1 component share many characteristics, the SonR1 may behave similarly when changing AV-delay [10]. Finally, the heart sounds contain components of closure of the RV and LV valves. In case of a dyssynchronous heart these components may not be 
synchronous and damp each other, while resynchronization may lead to better fusion of the mitral (M1) and tricuspid (T1) component, potentially increasing S1 amplitude.

The resultant of these three effects is not well understood. Therefore, the present study was meant to better understand the relation between the SonR1 signal and cardiac pump function in CRT applications. To this purpose, experiments were performed in dogs with synchronous and dyssynchronous heart failure. More specifically, three factors were investigated that may influence the relation between SonR1 and hemodynamic effect: 1) increased contractility, using dobutamine infusion; 2) atrioventricular coupling and 3) interventricular dyssynchrony.

\section{METHODS}

Animal handling was performed in compliance with the Guide for the Care and Use of Laboratory Animals and in accordance with the European Community recommendation. Experiments were performed at Institute Mutualiste Montsouris Recherche in Paris, France. The protocol was approved by the institutional ethics committee.

\section{Animal model}

Experiments were performed in 10 adult dogs of either sex and a bodyweight of $20-25 \mathrm{~kg}$.

\section{Induction of heart failure}

After induction with intravenous sodium thiopental, anesthesia was maintained by continuous infusion of midazolam $(0,25 \mathrm{mg} / \mathrm{kg} / \mathrm{h})$ and sufentanyl $(3 \mu \mathrm{g} / \mathrm{kg} / \mathrm{h})$. During a sterile closed chest procedure radiofrequency ablation (MarinR, Medtronic, Heerlen, the Netherlands) was used to create left bundle branch block (LBBB) [11]. Two SonRtip ${ }^{\mathrm{TM}}$ leads (Sorin CRM SAS, Clamart, France) were implanted in the right atrium (RA) and the RV apex. The leads were connected to a modified PARADYM ${ }^{\mathrm{TM}}$ RF SonR CRT-D pacemaker (Sorin CRM SAS, Clamart, France). In 6 animals tachypacing at the RV (180-200 bpm) for 4-5 weeks was used to induce heart failure. In one animal low contractility was induced in the acute setting using ventilation with isoflurane, one animal had spontaneous low contractility and in one animal heart failure was not induced.

\section{Final experiments}

Sacrifice experiments were performed after 4-5 weeks of tachypacing $(n=6)$ or in the acute setting using the same anesthesia as described above. RV and LV pressure were measured using 7F catheter-tip manometer catheters (CD-Leycom, Zoetermeer, the Netherlands). Two multi-electrode electrophysiological catheters (Medtronic, Heerlen, the Netherlands) were placed transvenously in two different cardiac veins via the coronary sinus for pacing and electrical mapping. Electrocardiograms (ECG) were recorded from the limb leads. Data were acquired at $1000 \mathrm{~Hz}$ using the IDEEQ Signal Analysis Software (Maastricht Instruments, Maastricht, the Netherlands). 
Baseline $(\mathrm{BL})$ measurements were performed during atrial pacing at a rate of $\sim 10 \mathrm{bpm}$ above the intrinsic rhythm. The total AV-block animals were paced at the RV lateral wall at an AV-delay of $150 \mathrm{~ms}$. Measurements were performed during variation of AV-delay in LV-only and BiV pacing mode. The AV-intervals were chosen to provide a range of various degrees of fusion between pacing-induced activation and intrinsic (right bundle branch (RBB) or RV lateral wall pacing) activation. In case of BiV pacing the $\mathrm{V}$-interval was set to zero. BL recordings were repeated after each pacing setting [12].

In three LBBB animals, cardiac contractility was enhanced using gradually increasing dobutamine infusion, with the maximal infusion rate being $40 \mu \mathrm{g} / \mathrm{kg} / \mathrm{min}$.

\section{Hemodynamic and SonR analysis}

Data analysis was performed using custom-made MATLAB software. Hemodynamic and accelerometer data were recorded and averaged for two respiratory cycles. Premature beats were excluded from the analysis. PR and QRS duration were measured from the surface ECG. LV maximal (LVP max $_{\text {max }}$ ) and end-diastolic pressures, and $\mathrm{dP} / \mathrm{dt}_{\max }$ were derived from LV and RV pressure signals. SonR signals were notch filtered before further analysis. SonR1 peak-to-peak amplitudes were calculated for RA and RV [6].

Mechanical interventricular dyssynchrony (MIVD) was determined as the time delay between the normalized upslope of simultaneously recorded LV and RV pressure curves [13]. The time between the moments of $L V$ and $R V \mathrm{dP} / \mathrm{dt}_{\text {max }}$ was calculated as an alternative for MIVD. A negative MIVD indicates that the RV is activated before the LV and is typical for LBBB and RV pacing.

The optimal AV-delay $\left(A V_{o p t}\right)$ was defined for each individual animal and for each pacing setting and defined as the AV-delay with the maximal relative increase in $\mathrm{LV} d P / \mathrm{dt}_{\max }$. The two shortest AV-delays for each animal were averaged and referred to as $A V_{\text {short }}$.

\section{Statistical analysis}

Continuous data are presented as mean \pm standard error of the mean (SEM). Paired and unpaired sampled t-tests were used for comparing dependent and independent continuous variables, respectively. Linear regression analysis was performed, and correlation coefficients are presented as $\mathrm{R}^{2}$. A two-sided probability value of $<0.05$ was considered statistically significant. A Bonferroni correction was applied when multiple groups were compared. Statistics were performed using Microsoft Excel (Microsoft, Redmond, WA). 


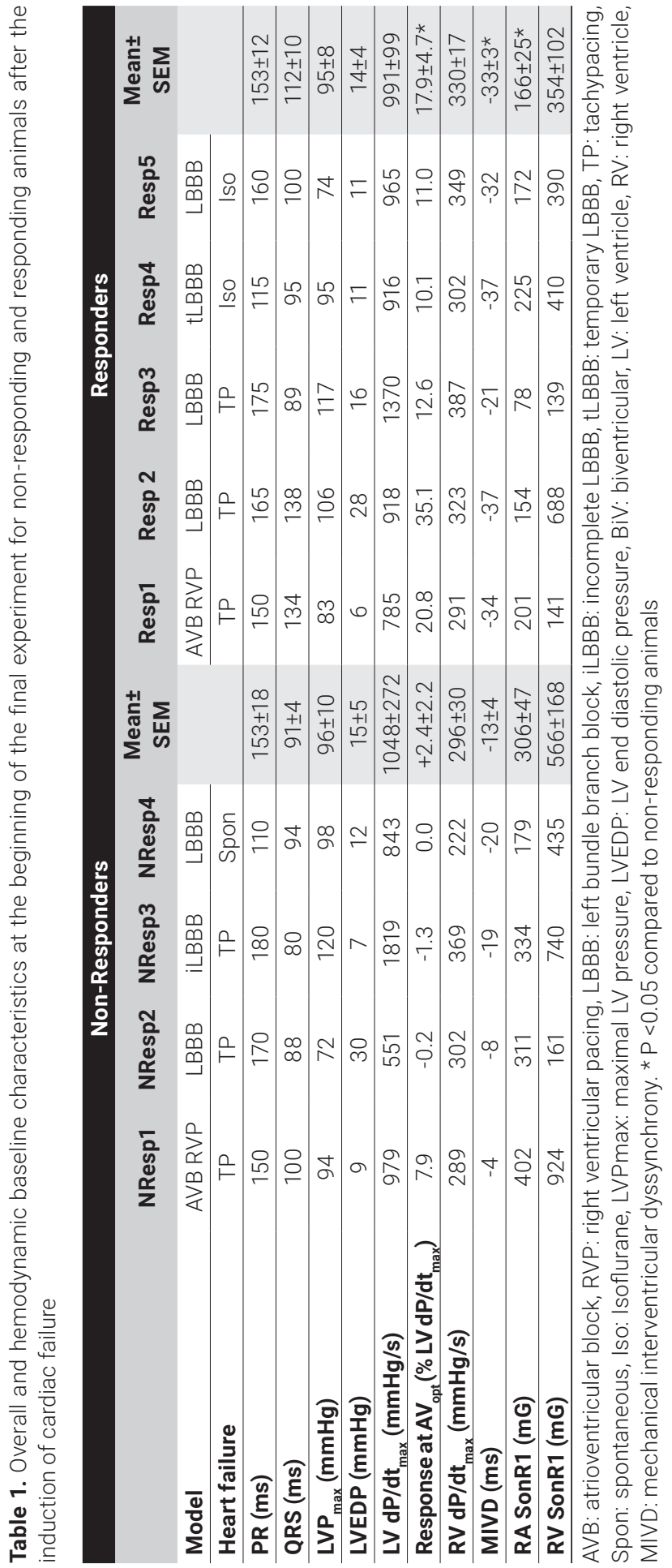




\section{RESULTS}

Of the 10 experiments performed, one was excluded from analysis because of premature death during the tachypacing period. LBBB creation was successful in six experiments, while in two experiments complete atrioventricular (AV) block developed (NResp1 and Resp1) and subsequently an RV lead was transvenously placed to mimic LBBB. In 1 animal LBBB creation was only temporary (Resp4). Heart failure was induced by tachypacing in 6 animals and isoflurane inhalation in 1 animal (Resp5). One animal was in heart failure without additional intervention (NResp4) and in 1 animal cardiac failure was induced using isoflurane only after the disappearance of the temporary LBBB (Resp4).

The animals were divided into two groups (see table 1) based on the maximal relative increase of LV dP/dt max $_{\text {ax }}$ during LV or BiV pacing with a cut off value of $>10 \%$. Table 1 shows

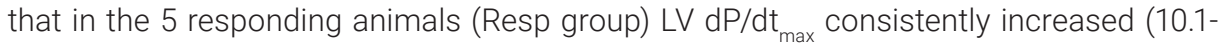
$35.1 \%$ ), whereas the 4 non-responding animals (NResp group) had negligible hemodynamic benefits from LV and BiV pacing (-1.3-7.9\%, $\mathrm{P}=0.023$ vs Resp animals). Additionally, the Resp animals were more dyssynchronous than the NResp animals indicated by a greater MIVD (-32 $\pm 3 m s$ vs $-13 \pm 4 m s$ respectively, $p=0.012)$. Animal NResp1 showed a synchronous contraction because the RV pacing lead was placed in an apical instead of lateral position. LBBB was incomplete in animal NResp3.

Baseline hemodynamic parameters, like LVP max LV end diastolic pressure and LV and RV $\mathrm{dP} / \mathrm{dt}_{\text {max }}$, were not significantly different between the NResp and Resp groups. Importantly, RA SonR1 and RV SonR1 were higher in the NResp animals, however only significantly for RA SonR1 (306 $\pm 47 m G$ vs 166 $\pm 25 m G$ for NResp and Resp animals). 


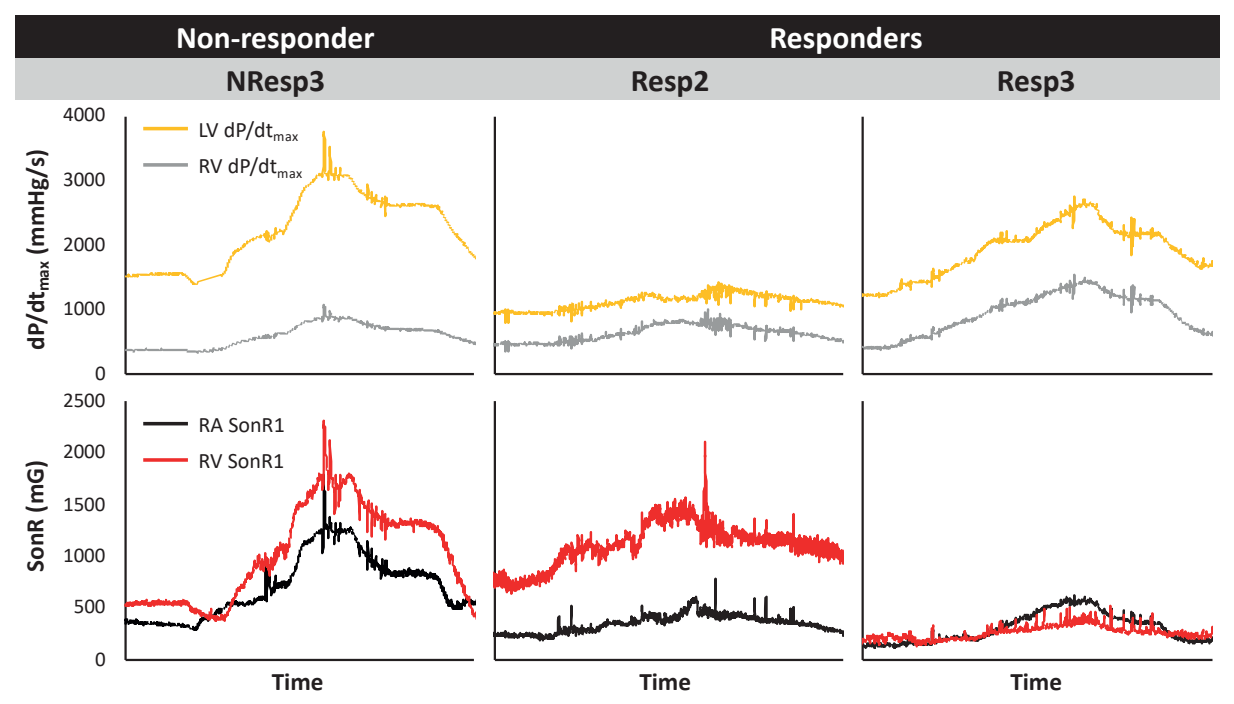

Figure 1. Dobutamine stress test in 3 animals. Concentrations of dobutamine infusion were gradually increased and subsequently decreased and stopped. The animals were in sinus rhythm, heart rate slightly decreases at maximal infusion rates. The upper panels present the changes in LV and RV $\mathrm{dP} / \mathrm{dt}_{\text {max }}$. In the lower panels the accompanied increases in RA and RV SonR1 are shown. Data are presented as moving average over 5 beats.

\section{The effect of contractility on SonR1}

Dobutamine stress tests were performed in 3 animals (Figure 1) to test the effect of contractility on SonR1 amplitude. Dobutamine reversibly increased cardiac contractility ( $L V$ and RV dP/dt ${ }_{\text {max }}$ ) by a factor 2-3. RA and RV SonR1 closely followed the changes in $\mathrm{dP} /$ $\mathrm{dt}_{\max }$ and also increased by a factor 2-3. Over the entire range of values strong correlations between LV and RV dP/dt $t_{\max }$ and RA and RV SonR1 were observed (Table 2); the correlation of SonR1 with RV $d P / d_{\text {max }}$ being slightly better.

Table 2. Correlation $\left(R^{2}\right)$ between $L V$ and $R V d P / d t_{\max }$ and RA and RV SonR1 during dobutamine stress test

\begin{tabular}{|c|c|c|c|c|c|}
\hline & & NResp1 & Resp2 & Resp3 & Mean $\pm S E M$ \\
\hline \multirow{2}{*}{ RA SonR1 } & $\mathrm{LV} \mathrm{dP/dt} \max _{\max }$ & 0.93 & 0.72 & 0.88 & $0.84 \pm 0.06$ \\
\hline & $\mathrm{RV} \mathrm{dP} / \mathrm{dt}_{\text {max }}$ & 0.95 & 0.83 & 0.89 & $0.89 \pm 0.04$ \\
\hline \multirow{2}{*}{ RV SonR1 } & $\mathrm{LV} \mathrm{dP/dt} \mathrm{max}_{\max }$ & 0.95 & 0.71 & 0.73 & $0.80 \pm 0.08$ \\
\hline & $\mathrm{RV} \mathrm{dP/dt} \mathrm{tax}_{\text {max }}$ & 0.96 & 0.82 & 0.70 & $0.83 \pm 0.07$ \\
\hline
\end{tabular}

$\mathrm{RA}$ : right atrium, $\mathrm{LV}$ : left ventricle, $\mathrm{RV}$ : right ventricle. 


\section{Hemodynamic effects of BiV pacing in Resp and NResp animals}

To investigate the effect of AV-delay on SonR1, data of NResp and Resp animals were compared during BiV pacing protocols. Values during the entire range of AV-delays are presented for representative NResp and Resp animals in figure $\mathbf{2}$ whereas values for all animals at $A V_{\text {short }}$ and $A V_{\text {opt }}$ are shown in figure $\mathbf{3}$.

In Resp animals BiV pacing tended to increase $\mathrm{LVP}_{\max }$ at $\mathrm{AV}_{\text {opt' }}$ while a non-significant decrease was seen during short AV-delays. LV end diastolic pressure remained relatively stable with changing $A V$-delays, with a trend to decrease at $A V_{\text {short }}$ In contrast, BiV pacing in the NResp animals did not change $L V P_{\text {max }}$ at $A V_{\text {opt }}$ and revealed a decrease at $A V_{\text {short' }}$ while no alteration in LV end diastolic pressure was observed (upper row figure $\mathbf{2}$ and $\mathbf{3}$ ).

In the Resp group all animals showed, by definition, an increase in LV dP/dt ${ }_{\max }$ compared to $B L$ values during BiV pacing at $A V_{\text {opt }}(1101 \pm 103 \mathrm{mmHg} / \mathrm{s}$ vs $987 \pm 117 \mathrm{mmHg} / \mathrm{s}$, respectively, $p=0.025$, second row figure 2), whereas NResp animals showed small and inconsistent changes (second row of figures 2 and 3). Peak increase of $L V d P / d t_{\max }$ in Resp animals occurred at intermediate AV-delays (60-150ms), while in NResp animals the highest (least decreased) values were observed at the longest AV-delays and a consistent decrease was seen at the shortest AV-delays. RV dP/dt ${ }_{\max }$ showed a less reproducible pattern, especially in the Resp group. In the NResp group a slight increase was observed at AVdelays ranging from 60-105ms (example in second row figure 2). BiV pacing at short AV-delays consistently decreased $\mathrm{RV} \mathrm{dP} / \mathrm{dt}_{\max }$ in all animals.

No changes in MIVD occurred when varying AV-intervals in BiV pacing in NResp animals (example in third row figure 2, group data in figure 3). In contrast, MIVD values became close to zero when shortening the AV-delay in Resp animals. A significant reduction in MIVD was observed at $\mathrm{AV}_{\text {opt }}$ during BiV pacing in de Resp group 


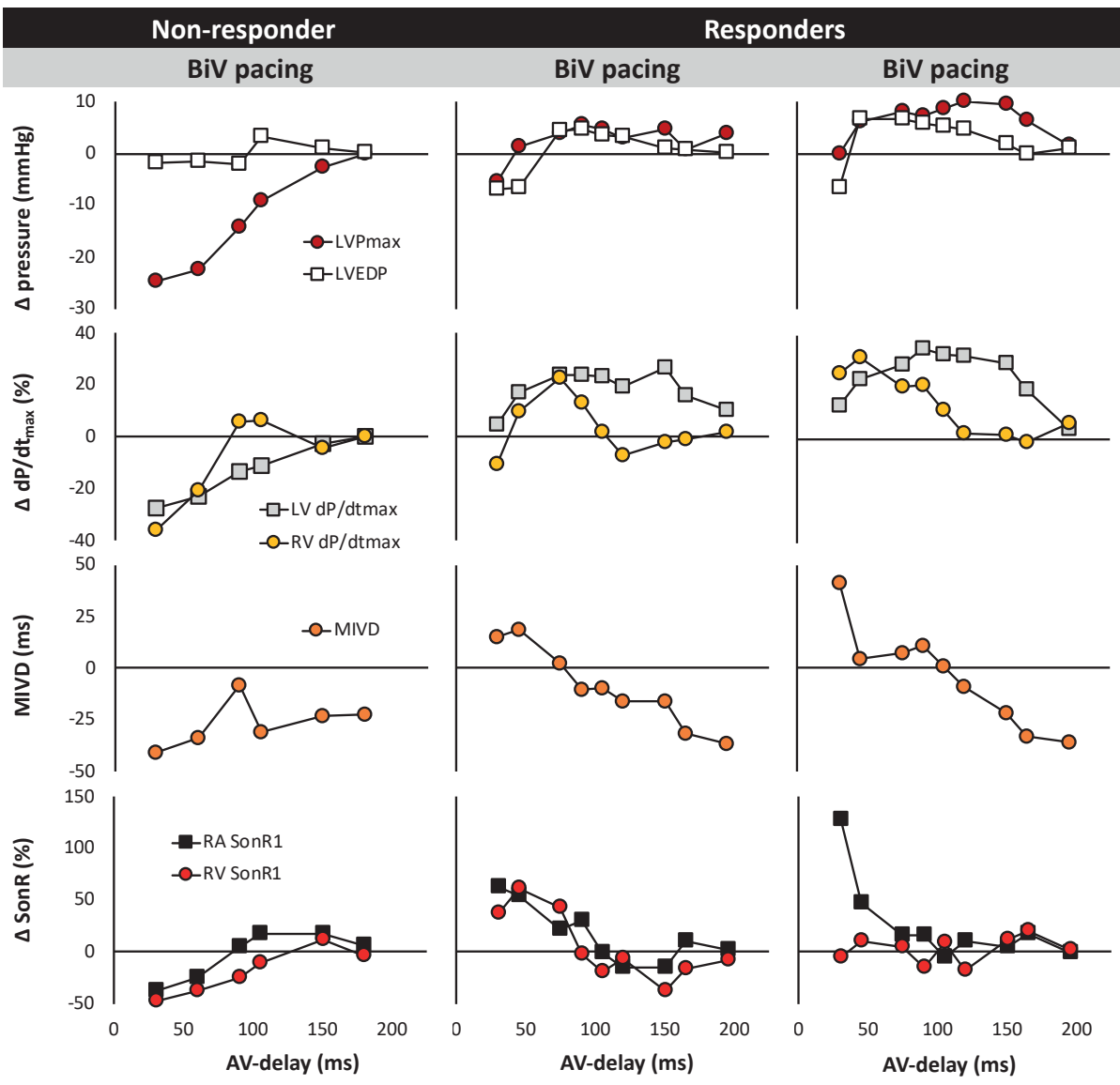

Figure 2. Typical examples of non-responding (left, NResp4) and responding animals (Resp2) during BiV (middle) and LV (right) pacing protocol. The upper row presents the absolute changes of LVP $P_{\max }$ and LVEDP. Relative change compared to BL values are given for LV and RV dp/dt $\mathrm{max}_{\text {max }}$ the second row and SonR in the last row. Absolute values are shown for MIVD in the third row. BiV: biventricular, LV: left ventricular, LVP max $_{\text {: }}$ maximal left ventricular pressure, LVEDP: left ventricular end diastolic pressure, MIVD: mechanical interventricular dyssynchrony, RA: right atrium, RV: right ventricle, BL: baseline.

SonR1 did not show any consistent changes in NResp animals (see example in figure 2 and group values in figure $\mathbf{3}$ lower left panel). In the Resp group BiV pacing at varying AVdelays resulted in a sigmoid-shaped curve with the highest values at the shortest AV-delays in 3 out of 4 animals for RA SonR1 (see example in figure 2). RV SonR1 showed a similar sigmoid shape in Resp animals, however with a smaller relative increase. All Resp animals showed higher relative increases of RA SonR1 at $A V_{\text {short }}$ compared to $\mathrm{AV}_{\text {opt }}(+129 \pm 77 \%$ vs $+77 \pm 30 \%$ respectively), whereas RV SonR1 values did not consistently change between $A V_{\text {opt }}$ and $A V_{\text {short }}$ (bottom panels figure 3 ). While the absolute values of RA SonR1 were lower than those of RV SonR1 (Table 1), the relative increase of RA SonR1 was significantly 
larger than that of RV SonR1 during BiV pacing at $\mathrm{AV}_{\text {opt }}(+77 \pm 30 \%$ vs $+25 \pm 27 \%$ respectively, $\mathrm{P}=0.033)$.

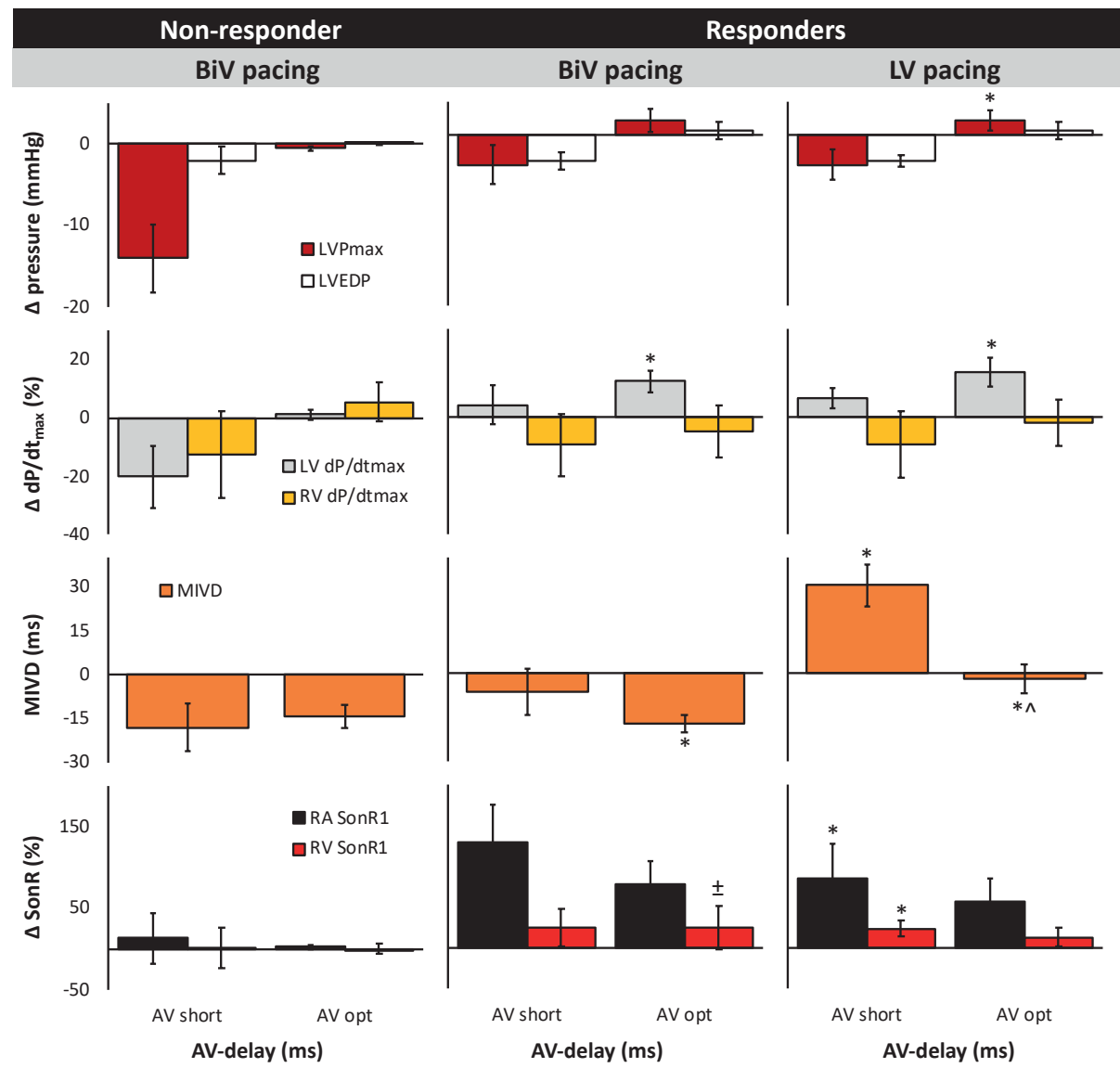

Figure 3. Overall mean values of non-responding and responding animals during BiV (left/middle) and $L V$ (right) pacing at short $\left(A V_{\text {short }}\right)$ and optimal $A V$-delays $\left(A V_{\text {opt }}\right)$. $A V_{\text {short }}$ is the mean value of the two shortest AV-delays. AVopt was defined as the AV-delay with the largest relative increase of LV dP/ $\mathrm{dt}_{\max }$. The upper row presents the absolute changes of LVP ${ }_{\max }$ and LVEDP. Relative change compared to $B L$ values are given for $L V$ and $R V d p / d t_{\max }$ in the second row and SonR in the last row. Absolute values are shown for MIVD and the time between $\mathrm{LV}$ and $\mathrm{RV} \mathrm{dP} / \mathrm{dt}_{\text {max }}$ in the third row. * indicates $\mathrm{P}<0.05$ compared to $B L$ values, ${ }^{\wedge}$ indicates $P<0.05$ compared to short AV-delay, and ${ }^{ \pm}$indicates $P<0.05$ between RA and RV SonR1. Abbreviations as in figure 2.

\section{Comparison of BiV and LV pacing in dyssynchronous hearts}

To evaluate the effect of interventricular dyssynchrony on the SonR signal, the effects of BiV and LV pacing were compared in the Resp animals. The LV pacing data are presented in the right panels of figures $\mathbf{2}$ and $\mathbf{3}$. LVP $P_{\max }$ and LV end diastolic pressure showed the same behavior during BiV and LV pacing, with the largest increase at intermediate AVdelays. During LV pacing at $A V_{\text {opt }} L V P_{\text {max }}$ was significantly higher than at $B L(102 \pm 9 \mathrm{mmHg}$ 
vs $97 \pm 9 \mathrm{mmHg}$ respectively, $P=0.024)$. The parabolic pattern of $L V d P / d t_{\text {max }}$ that was visible during BiV pacing was also observed during LV pacing (second row figure $\mathbf{2}$ and $\mathbf{3}$ ). At $A V_{\text {opt }}$ $\mathrm{LV} \mathrm{dP/dt}$ max was, by definition, significantly elevated compared to BL values $(+15.1 \pm 5.0 \%$, $p=0.013)$. No significant differences were observed between BiV and LV pacing for the aforementioned parameters.

Pacing at $A V_{\text {short' }}$ and thereby fully capturing of LV and BiV pacing, led to positive MIVD values during $L V$ pacing $(+30 \pm 7 m s)$, indicating $L V$ preexcitation, whereas BiV pacing created MIVD values close to zero $\left(-6 \pm 8 \mathrm{~ms}, \mathrm{P}=0.043\right.$ compared to $L V$ pacing). MIVD at $A V_{\text {short }}$ $(+30 \pm 7 \mathrm{~ms})$ and $A V_{\text {opt }}(-2 \pm 5 \mathrm{~ms})$ differed significantly to $B L$ values $(-33 \pm 3 \mathrm{~ms}, \mathrm{P}=0.001$ for $\mathrm{AV}_{\text {short }}$ and $\mathrm{P}=0.000$ for $\mathrm{AV}_{\text {opt }}$ ) during LV pacing.

The behavior of RA SonR1 amplitude was similar between BiV and LV pacing, with a sigmoid curve (Figure 2) and a maximal increase during LV pacing at $A V_{\text {short }}(279 \pm 23 \mathrm{mG}$ at $A V_{\text {short }}$ vs $174 \pm 30 \mathrm{mG}$ at $B L, P=0.012$, figure 3 bottom right panel). During $A V_{\text {opt }}$ and $A V_{\text {short }}$ both RA and RV SonR1 were not significantly different between BiV and LV pacing, despite a significant difference in MIVD between these pacing modes.

\section{DISCUSSION}

This experimental study shows that the amplitude of SonR1 is determined by contractility and time between atrial and ventricular contraction. During BiV pacing at intermediate AV-delays, SonR1 increases in parallel with an increase in systolic LV pressure and LV $\mathrm{dP} / \mathrm{dt}_{\max }$ and distinguishes between animals responding and not responding to CRT. Interventricular dyssynchrony is not a major determinant of SonR1, because during LV and BiV pacing similar LV dP/dt max $_{\text {ax }}$ and SonR1 changes occurred while MIVD was clearly different. In hemodynamic responders, SonR1 continues to increase during BiV and LV pacing at shorter AV-delays, despite a reduction in LV pressure and LV dP/dt ${ }_{\max }$. The latter increase in SonR1 seems caused by a short time interval between atrial and ventricular contraction. These data support the use of the inflection point of the AV-delay - SonR1 curve for automatic programming of AV-delay in CRT. Although absolute values of RA SonR1 were smaller than those of RV SonR1, RA SonR1 appears more sensitive to find the hemodynamic optimum.

\section{SonR1 and cardiac contractility}

The dobutamine stress data in this study support previous data that SonR1 reflects contractility in hearts when activation pattern and AV-delay were kept constant. Bordachar et al. showed that during dobutamine infusion in pigs with normal and ischemia-induced decreased LV function changes of transcutaneous SonR1 amplitude correlated with LV dP/ $\mathrm{dt}_{\max }[14]$. Similar results were found with an accelerometer embedded in the atrial pacing lead [8, 15-17]. Our data also support earlier findings in patients and sheep that in the setting of dobutamine stress test the SonR1 amplitude reflects both LV and RV contractility [6]. The contribution of both ventricles can be explained by the fact that both ventricles are 
involved in the SonR1 signal, because the myocardial vibrations that are considered to be responsible for the heart sounds are produced by the RV valves as well.

This phenomenon can be further understood from the resemblance between the SonR signal and the phonocardiogram. In 1963 Shah et al. showed that the second component of the first heart sound (S1) as derived from intracavitary and external phonocardiograms coincided with the peak of LV pressure rise $\left(\mathrm{LV} d P / \mathrm{dt}_{\max }\right)$ in dogs [18]. Subsequently, the amplitude of S1 seemed to show a strong linear correlation to LV $\mathrm{dP} / \mathrm{dt}_{\max }$ over a wide range of pressures, volumes and cardiodynamics, suggesting that S1 amplitude is mainly determined by cardiac contractility under these conditions [19].

The origin and significance of the different components of the first heart sound are considered to come from a M1 and T1 component. Some studies seem to indicate that mitral valve closure has the largest contribution to S1, but in this study the sensor was positioned in the LV [20]. Instead, in our study, with the sensor positioned in the RA and $\mathrm{RV}$, the dobutamine data show a slightly higher correlation of SonR1 with RV than with $\mathrm{LV} d \mathrm{P} / \mathrm{dt}_{\max }$.

\section{AV-delay optimization during resynchronization}

To the best of our knowledge, the present study is the first to validate SonR1 against

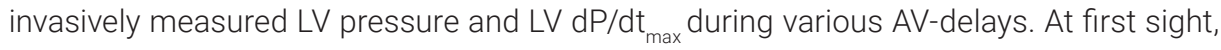
the link between SonR1 and LV dP/dt max $_{\text {max }}$ during BiV and LV pacing in Resp animals seems comparable with that during the dobutamine stress test. However, several differences are present.

First of all, the relative change in $\mathrm{LV} \mathrm{dP} / \mathrm{dt}_{\max }$ is much smaller during pacing $(\sim 10 \%)$ than during dobutamine (100-200\%) and so are changes in SonR1 (up to 80\% for RA SonR1 and $\sim 20 \%$ for RV SonR1 for pacing as compared to 100-200\% during dobutamine). These numbers do point out that the SonR1 signal seems more sensitive to pacing-induced resynchronization than to dobutamine-induced increase in contractility. The latter is driven by better calcium handling of the myocardium, whereas resynchronization creates better mechanical coordination between ventricular wall segments.

Secondly, while RA and RV SonR1 show similar relative increases during dobutamine stress test, during resynchronization (BiV and LV pacing during intermediate AV-delays) the relative RV SonR1 increase is only a quarter of that of RA SonR. A possible explanation for this observation is that the RV sensor primarily senses RV processes, whereas the RA sensor, although at larger distance of the sources of the acceleration (hence the lower amplitudes), senses changes at both ventricles. The change in RV SonR1 may be smaller because during resynchronization $\mathrm{RV} \mathrm{dP} / \mathrm{dt}_{\max }$ does not increase and has even the tendency to decrease. RV SonR1 performs well during dobutamine testing, presumably because dobutamine increases RV and LV contractility to a similar extent. 
Thirdly, an increase in SonR1 when pacing at short AV-delays has been attributed to atrial contraction against a closed or quickly closing mitral valve. Shah et al. have shown that S1 amplitudes were larger at high speed closure of the mitral valve in patients with complete heart block and various intervals between atrial and ventricular systole [21]. Stept et al. reported that the amplitude of S1 significantly incremented at short PR-intervals while $\mathrm{LV} \mathrm{dP/dt}{ }_{\max }$ did not change compared to those at longer PR-intervals in a healthy dog model [22]. However, in our study such SonR1 increase only occurs in resynchronized, responding animals. This may be explained by the considerable drop in LV contractility and systolic pressure when pacing at short AV-delay in the NResp animals. Apparently, a large SonR1 at short AV-delays is only obtained when, due to resynchronization, ventricular pump function is fairly well maintained when the atrium contracts against a closed mitral valve. Whatever the exact mechanism of the large SonR1 signals is, these data indicate that SonR1 amplitude is sensitive to the interval between atrial and ventricular systole (in the present study modified by changing paced AV-delay).

\section{The use of SonR1 for ambulatory AV optimization}

Programming an optimal AV-delay has been an issue of continuous debate. Several studies using 'static' (i.e. a single, in rest) AV optimization were not able to show a long-term benefit of CRT as compared to using a default setting [23-25]. However, acute hemodynamic studies have shown benefit of CRT optimization and that the optimal AV-delay varies from one patient to another and may change between rest and exercise [26-29].

Dynamic, automated algorithms for AV optimization have been developed, but except for the SonR system, all are based on electrograms, so using an electrical signal. The Adaptive CRT algorithm provides LV-only pacing timed with native RV activation when the AV-interval is normal, optimizing $A V$ and $V V$-delay on the basis of periodic automatic evaluation of intrinsic conduction intervals $[24,30]$. A recent study in HF patient with normal AV-conduction, without atrial fibrillation (AF) and with an indication for CRT showed that LV-only pacing timed with native RV activation may result in greater improvements in LV ejection fraction and myocardial strain compared with BiV pacing due to better apical and septal function [31]. The SyncAV algorithm provides a concept of BiV pacing by periodically measuring intrinsic conduction and dynamically adjusting AV-delays $50 \mathrm{~ms}$ shorter than the intrinsic AV-interval, allowing for paced BiV wavefronts to fuse with intrinsic conduction (triple wavefront fusion) [32, 33]. Compared to QuickOpt optimization, SyncAV CRT led to narrower QRS complexes and higher aortic VTI in HF patients with a class I indication for CRT [34].

It is the strength of the SonR system that it uses a mechanical sensor, so far the only clinically applied one for this purpose. Ritter et al. were the first to use the SonR signal to obtain a tailored AV-delay that correlated closely to AV-delays as determined by echocardiography in patients with high degree AV-block paced in DDD mode [35]. Also in total AV-block patients receiving CRT RV SonR1 amplitude correlated significantly with 
changes in aortic velocity time integral (VTI) as a measure of cardiac function [7]. Later the SonR algorithm was further optimized by Dupuis et al. to better predict the optimal AV-delay, which was qualified as the shortest AV-delay where the mitral valve closes in physiological fashion without truncation of the A-wave of the flow velocity curve [36, 37]. This moment appeared to correspond with the shortest AV-delay without significant rise in SonR1 amplitude, the so-called inflection point of the sigmoid SonR curve.

We did not systematically determine the inflection point. However, figure $\mathbf{2}$ shows that during BiV and LV pacing the highest values of $L V P_{\max }$ and $L V d P / d t_{\max }$ occur at AV-delays slightly longer than the one where SonR1 rises.

\section{Interventricular dyssynchrony}

The present data indicate that interventricular dyssynchrony is not a major determinant of SonR1. This conclusion may be drawn from the high degree of similarity of SonR1 values during BiV and LV pacing in Resp animals. This finding seems to be in contrast with the advised use of the current SonR algorithm. This algorithm prescribes to first apply an AVdelay scan at five different $\mathrm{V} V$-delays and then use the $\mathrm{V} V$-delay with the highest average SonR1 value for finding the optimal AV-delay, as quantified by the 'PEA area ' [38]. In the latter study the optimal AV-delays defined by LV dP/dt max and SonR1 (PEA called at that time) were concordant in 9 out of 12 patients. On the other hand, $P E A_{\text {area }}$ was not much different between the various $\mathrm{V} V$-delays, which seems to support the data from the present study.

\section{Position of SonR accelerometer}

The current experiment was performed with SonR leads located in the RA and RV. Initially the accelerometer was placed in the RV-lead, however in the clinical setting the switch has been made to the RA [15-17, 39]. Previous research using a temporary accelerometer in patients showed a higher SonR signal in the RV compared to the RA during sinus rhythm and atrial fibrillation [40]. In our study absolute values of RV SonR1 were indeed higher than RA SonR1 signals, but relative increases were more pronounced in RA SonR1 signals, especially during short AV-delays with early mitral flow reversal. This might possibly lead to more turbulence in the atria leading to greater vibrations and higher RA SonR1 values. We hypothesize that the placement of the SonR accelerometer in the RA gives a better overview of the overall cardiac function, including that of the left ventricle, while placing the SonR1 sensor in the RV might lead to a greater contribution of RV contractility.

\section{Potential clinical applications}

Currently the SonR algorithm is being used in a biventricular implantable cardiac defibrillator (CRT-D) system with the SonR sensor embedded in the atrial pacing lead (PARADYM ${ }^{T M}$ RF SonR CRT-D). A randomized pilot study, The Clinical Evaluation of Advanced Resynchronization (CLEAR) trial, has shown that RA SonR1 based optimization of CRT in heart failure (HF) patients significantly improved on New York Heart Association (NYHA) functional class, with fewer deaths and fewer hospitalizations compared to 
standard echocardiographic optimization [41]. More recently the RESPOND-CRT trial, a prospective, multicenter, randomized, double-blind study in 1039 patients with systolic HF and wide QRS complex, showed that RA SonR1 based optimization of CRT is non-inferior to standard echocardiographic optimization in terms of responder rate and results in a $35 \%$ risk reduction for heart failure hospitalization $[42,43]$.

The current animal study provides support for the use of the SonR system and provides a better understanding of the pathophysiology of the signal. Although the present study is small, several findings may be worthwhile for further investigation in order to further improve the SonR algorithm:

- Our study has shown that the SonR1 signal is suitable for AV-delay optimization during LV-only pacing. This indicates that the SonR1 algorithm might be used for optimizing LV fusion pacing, a pacing mode that has been shown superior to biventricular pacing when using the adaptive CRT algorithm $[44,45]$.

- The present study shows some variability in the SonR data, as evidenced from the AV-scans shown in figure 2 . This variability hampers accurate determination of the inflection point. This may be circumvented by using a longer sampling time. Alternatively, the data show that the exact point is not important, as long as SonR1 does not increase exponentially, as is the case during the short AV-delays. So using an AV-delay that is just several tens of milliseconds longer than the range where the exponential increase is seen seems to be sufficient.

\section{Future perspectives}

The SonR1 peak-to-peak amplitude is just one simple characteristic of the acquired SonR signal. It may well be that there is complementary information in the SonR signals, like the width of SonR1, the time delay between the M1 and $\mathrm{T} 1$ components as well as the time between onset of QRS complex and S1 (elsewhere referred to as electromechanical activation time or EMAT [46]). In addition, also the SonR equivalent of the second heart sound, S2, may contain useful information such as the amount of splitting of S2 (timing difference between the closure of the aortic (A2) and pulmonary (P2) valves. Finally, optimal diastolic filling (leading to enhanced systolic function), can be calculated from SonR1 and SonR2 components since they appear to correlate to mitral and aortic valve closure [47]. Therefore, it seems worthwhile to design future studies, searching for even more comprehensive algorithms for hemodynamic optimization, such as multiparameter analysis of the accelerometer signals.

\section{Limitations}

Main limitation of this study is the small number of animals and the variability in conduction abnormalities. On the other hand, the fact that a relation was found between SonR1 on the one hand and contractility and AV-delay on the other, despite this variability, indicates that this study provides better understanding of the origin of the SonR1 signal. Further support for the ideas unfolded here require additional experiments. Results from animal 
experiments need to be extrapolated with care to the clinical situation. Importantly also, acute hemodynamic improvements may not (fully) translate into clinical improvements. However, the positive results of the CLEAR and RESPOND-CRT trials are promising in this respect.

\section{CONCLUSIONS}

The SonR1 amplitude is a complex parameter that is influenced by changes in cardiac contractility and AV-delay dependent alterations of transmitral flow. Although the SonR algorithm is an effective way of optimizing $A V$ and $V V$-delay in patients receiving $C R T$, the pathophysiology of the SonR1 signal needs further unravelling in order to optimize the algorithm. 


\section{REFERENCES}

1 Zanon F, Baracca E, Pastore G, Marcantoni L, Fraccaro C, Lanza D, et al. Multipoint pacing by a left ventricular quadripolar lead improves the acute hemodynamic response to CRT compared with conventional biventricular pacing at any site. Heart Rhythm. 2015.

2 Curtis AB, Worley SJ, Adamson PB, Chung ES, Niazi I, Sherfesee L, et al. Biventricular pacing for atrioventricular block and systolic dysfunction. N Engl J Med. 2013;368(17):1585-93.

3 Prinzen FW, Vernooy K, Auricchio A. Cardiac resynchronization therapy: state-of-the-art of current applications, guidelines, ongoing trials, and areas of controversy. Circulation. 2013;128(22):2407-18.

4 Houthuizen P, Bracke FA, van Gelder BM. Atrioventricular and interventricular delay optimization in cardiac resynchronization therapy: physiological principles and overview of available methods. Heart Fail Rev. 2011;16(3):263-76.

5 Rushmer R. Cardiovascular dynamics: Saunders; 1976.

6 Rickards AF, Bombardini T, Corbucci G, Plicchi G. An implantable intracardiac accelerometer for monitoring myocardial contractility. The Multicenter PEA Study Group. Pacing Clin Electrophysiol. 1996;19(12 Pt 1):2066-71.

7 Bordachar P, Garrigue S, Reuter S, Hocini M, Kobeissi A, Gaggini G, et al. Hemodynamic assessment of right, left, and biventricular pacing by peak endocardial acceleration and echocardiography in patients with end-stage heart failure. Pacing Clin Electrophysiol. 2000;23(11 Pt 2):1726-30.

8 Plicchi G. PEA I and PEA II based implantable haemodynamic monitor: pre clinical studies in sheep. Europace. 2002;4(1):49-54.

9 Siecke $\mathrm{HEH}$. Relation of the difference in pressure across the mitral value to the amplitude of the first heart sound in dogs with atrioventricular block. Am J Physiol. 1958;192(1):135-40.

10 Tassin A, Kobeissi A, Vitali L, Rouleau F, Ritter P, Gaggini G, et al. Relationship between amplitude and timing of heart sounds and endocardial acceleration. Pacing Clin Electrophysiol. 2009;32 Suppl 1:S101-4.

11 Verbeek XA, Vernooy K, Peschar M, Cornelussen RN, Prinzen FW. Intra-ventricular resynchronization for optimal left ventricular function during pacing in experimental left bundle branch block. J Am Coll Cardiol. 2003;42(3):558-67.

12 Whinnett ZI, Francis DP, Denis A, Willson K, Pascale P, van Geldorp I, et al. Comparison of different invasive hemodynamic methods for AV delay optimization in patients with cardiac resynchronization therapy: implications for clinical trial design and clinical practice. Int J Cardiol. 2013;168(3):2228-37.

13 Verbeek XA, Vernooy K, Peschar M, Van Der Nagel T, Van Hunnik A, Prinzen FW. Quantification of interventricular asynchrony during LBBB and ventricular pacing. Am J Physiol Heart Circ Physiol. 2002;283(4):H1370-8.

14 Bordachar P, Labrousse L, Ploux S, Thambo JB, Lafitte S, Reant P, et al. Validation of a new noninvasive device for the monitoring of peak endocardial acceleration in pigs: implications for optimization of pacing site and configuration. J Cardiovasc Electrophysiol. 2008;19(7):725-9.

15 Bordachar P, Garrigue S, Ritter P, Ploux S, Labrousse L, Casset C, et al. Contributions of a hemodynamic sensor embedded in an atrial lead in a porcine model. J Cardiovasc Electrophysiol. 2011;22(5):579-83.

16 Bombardini T, Gaggini G, Marcelli E, Parlapiano M, Plicchi G. Peak endocardial acceleration reflects heart contractility also in atrial fibrillation. Pacing Clin Electrophysiol. 2000;23(9):1381-5.

17 Gras D, Kubler L, Ritter P, Anselme F, Delnoy PP, Bordachar P, et al. Recording of peak endocardial acceleration in the atrium. Pacing Clin Electrophysiol. 2009;32 Suppl 1:S240-6.

18 Shah PM, Mori M, MacCanon DM, Luisada AA. Hemodynamic correlates of the various components of the first heart sound. Circ Res. 1963;12:386-92.

19 Sakamoto T, Kusukawa R, Maccanon DM, Luisada AA. Hemodynamic determinants of the amplitude of the first heart sound. Circ Res. 1965;16:45-57. 
20 Luisada AA, MacCanon DM, Kumar S, Feigen LP. Changing views on the mechanism of the first and second heart sounds. Am Heart J. 1974;88(4):503-14.

21 Shah PM, Kramer DH, Gramiak R. Influence of the timing of atrial systole on mitral valve closure and on the first heart sound in man. Am J Cardiol. 1970;26(3):231-7.

22 Stept ME, Heid CE, Shaver JA, Leon DF, Leonard JJ. Effect of altering P-R interval on the amplitude of the first heart sound in the anesthetized dog. Circ Res. 1969;25(3):255-63.

23 Zhang Y, Xing Q, Zhang JH, Jiang WF, Qin M, Liu X. Long-Term Effect of Different Optimizing Methods for Cardiac Resynchronization Therapy in Patients with Heart Failure: A Randomized and Controlled Pilot Study. Cardiology. 2019;142(3):158-66.

24 Martin DO, Lemke B, Birnie D, Krum H, Lee KL, Aonuma K, et al. Investigation of a novel algorithm for synchronized left-ventricular pacing and ambulatory optimization of cardiac resynchronization therapy: results of the adaptive CRT trial. Heart Rhythm. 2012;9(11):1807-14.

25 Abraham WT, Gras D, Yu CM, Calo L, Islam N, N K. Results from the FREEDOM trial: assess the safety and efficacy of frequent optimization of cardiac resynchronization therapy. Presented at Heart Rhythm Society 31st Annual Scientific Sessions Denver. 2010.

26 Auricchio A, Stellbrink C, Sack S, Block M, Vogt J, Bakker P, et al. The Pacing Therapies for Congestive Heart Failure (PATH-CHF) study: rationale, design, and endpoints of a prospective randomized multicenter study. Am J Cardiol. 1999;83(5B):130D-5D.

27 Bogaard MD, Kirkels JH, Hauer RN, Loh P, Doevendans PA, Meine M. Should we optimize cardiac resynchronization therapy during exercise? J Cardiovasc Electrophysiol. 2010;21(11):1307-16.

28 Zweerink A, Salden OAE, van Everdingen WM, de Roest GJ, van de Ven PM, Cramer MJ, et al. Hemodynamic Optimization in Cardiac Resynchronization Therapy: Should We Aim for dP/ dtmax or Stroke Work? JACC Clin Electrophysiol. 2019;5(9):1013-25.

29 Whinnett ZI, Davies JE, Willson K, Manisty CH, Chow AW, Foale RA, et al. Haemodynamic effects of changes in atrioventricular and interventricular delay in cardiac resynchronisation therapy show a consistent pattern: analysis of shape, magnitude and relative importance of atrioventricular and interventricular delay. Heart. 2006;92(11):1628-34.

30 Krum H, Lemke B, Birnie D, Lee KL, Aonuma K, Starling RC, et al. A novel algorithm for individualized cardiac resynchronization therapy: rationale and design of the adaptive cardiac resynchronization therapy trial. Am Heart J. 2012;163(5):747-52 e1.

31 Burns KV, Gage RM, Curtin AE, Gorcsan J, 3rd, Bank AJ. Left ventricular-only pacing in heart failure patients with normal atrioventricular conduction improves global function and left ventricular regional mechanics compared with biventricular pacing: an adaptive cardiac resynchronization therapy sub-study. Eur J Heart Fail. 2017;19(10):1335-43.

32 Thibault B, Ritter P, Bode K, Calo L, Mondesert B, Mangual JO, et al. Dynamic programming of atrioventricular delay improves electrical synchrony in a multicenter cardiac resynchronization therapy study. Heart Rhythm. 2019;16(7):1047-56.

33 Varma N, O'Donnell D, Bassiouny M, Ritter P, Pappone C, Mangual J, et al. Programming Cardiac Resynchronization Therapy for Electrical Synchrony: Reaching Beyond Left Bundle Branch Block and Left Ventricular Activation Delay. J Am Heart Assoc. 2018;7(3).

34 Wang J, Liang Y, Chen H, Wang W, Bai J, Chen X, et al. Patient-tailored SyncAV algorithm: A novel strategy to improve synchrony and acute hemodynamic response in heart failure patients treated by cardiac resynchronization therapy. J Cardiovasc Electrophysiol. 2020;31(2):512-20.

35 Ritter P, Padeletti L, Gillio-Meina L, Gaggini G. Determination of the optimal atrioventricular delay in DDD pacing. Comparison between echo and peak endocardial acceleration measurements. Europace. 1999;1(2):126-30.

36 Dupuis JM, Kobeissi A, Vitali L, Gaggini G, Merheb M, Rouleau F, et al. Programming optimal atrioventricular delay in dual chamber pacing using peak endocardial acceleration: comparison with a standard echocardiographic procedure. Pacing Clin Electrophysiol. 2003;26(1 Pt 2):210-3.

37 Padeletti L, Porciani MC, Ritter P, Michelucci A, Colella A, Pieragnoli P, et al. Atrioventricular interval optimization in the right atrial appendage and interatrial septum pacing: a comparison between echo and peak endocardial acceleration measurements. Pacing Clin Electrophysiol. 2000;23(11 Pt 1):1618-22. 
38 Delnoy PP, Marcelli E, Oudeluttikhuis H, Nicastia D, Renesto F, Cercenelli L, et al. Validation of a peak endocardial acceleration-based algorithm to optimize cardiac resynchronization: early clinical results. Europace. 2008;10(7):801-8.

39 Duncker D, Delnoy PP, Nagele H, Mansourati J, Mont L, Anselme F, et al. First clinical evaluation of an atrial haemodynamic sensor lead for automatic optimization of cardiac resynchronization therapy. Europace. 2015.

40 Bongiorni MG, Soldati E, Arena G, Quirino G, Vernazza F, Bernasconi A, et al. Is local myocardial contractility related to endocardial acceleration signals detected by a transvenous pacing lead? Pacing Clin Electrophysiol. 1996;19(11 Pt 2):1682-8.

41 Ritter P, Delnoy PP, Padeletti L, Lunati M, Naegele H, Borri-Brunetto A, et al. A randomized pilot study of optimization of cardiac resynchronization therapy in sinus rhythm patients using a peak endocardial acceleration sensor vs. standard methods. Europace. 2012;14(9):1324-33.

42 Brugada J, Brachmann J, Delnoy PP, Padeletti L, Reynolds D, Ritter P, et al. Automatic optimization of cardiac resynchronization therapy using SonR-rationale and design of the clinical trial of the SonRtip lead and automatic AV-VV optimization algorithm in the paradym RF SonR CRT-D (RESPOND CRT) trial. Am Heart J. 2014;167(4):429-36.

43 Brugada J, Delnoy PP, Brachmann J, Reynolds D, Padeletti L, Noelker G, et al. Contractility sensor-guided optimization of cardiac resynchronization therapy: results from the RESPONDCRT trial. Eur Heart J. 2016.

44 AlTurki A, Lima PY, Bernier ML, Garcia D, Vidal A, Toscani B, et al. Optimization of Chronic Cardiac Resynchronization Therapy Using Fusion Pacing Algorithm Improves Echocardiographic Response. CJC Open. 2020;2(2):62-70.

45 Burri H, Prinzen FW, Gasparini M, Leclercq C. Left univentricular pacing for cardiac resynchronization therapy. Europace. 2017;19(6):912-9.

46 Hasan A, Abraham WT, Quinn-Tate L, Brown L, Amkieh A. Optimization of cardiac resynchronization devices using acoustic cardiography: a comparison to echocardiography. Congest Heart Fail. 2006;12 Suppl 1:25-31.

47 Giorgis L, Frogerais P, Amblard A, Donal E, Mabo P, Senhadji L, et al. Optimal algorithm switching for the estimation of systole period from cardiac microacceleration signals (SonR). IEEE Trans Biomed Eng. 2012;59(11):3009-15. 




\section{ABSTRACT}

\section{Introduction}

Timing of atrial, right (RV) and left ventricular (LV) stimulation in cardiac resynchronization therapy (CRT) is known to affect electrical activation and pump function of the LV. In this study, we used computer simulations, with input from animal experiments, to investigate the effect of varying pacing delays on both LV and RV electrical dyssynchrony and contractile function.

\section{Methods}

A pacing protocol was performed in dogs with atrioventricular block $(\mathrm{N}=6)$, using 100 different combinations of atrial (A)-LV and A-RV pacing delays. Regional LV and RV electrical activation times were measured using 112 electrodes and LV and RV pressures were measured with catheter-tip micromanometers. Contractile response to a pacing delay was defined as relative change of the maximum rate of $L V$ and $R V$ pressure rise $\left(\mathrm{dP}_{\mathrm{dt}} \mathrm{dt}_{\max }\right)$ compared to RV pacing with an A-RV delay of $125 \mathrm{~ms}$. The pacing protocol was simulated in the CircAdapt model of cardiovascular system dynamics, using the experimentally acquired electrical mapping data as input.

\section{Results}

Ventricular electrical activation changed with changes in the amount of LV or RV preexcitation. The resulting changes in $\mathrm{dP} / \mathrm{dt}_{\max }$ differed markedly between the LV and RV. Pacing the LV 10-50ms before the RV led to the largest increases in LV dP/dt ${ }_{\text {max }}$. In contrast, $\mathrm{RV} d \mathrm{dP} / \mathrm{dt}_{\max }$ was highest with RV pre-excitation and decreased up to $33 \%$ with LV preexcitation. These opposite patterns of changes in RV and LV dP/dt $t_{\max }$ were reproduced by the simulations. The simulations extended these observations by showing that changes in steady-state biventricular cardiac output differed from changes in both LV and RV dP/ $\mathrm{dt}_{\max }$. The model allowed to explain the discrepant changes in $\mathrm{dP} / \mathrm{dt}_{\max }$ and cardiac output by coupling between atria and ventricles as well as between the ventricles.

\section{Conclusions}

The LV and the RV respond in an opposite manner to variation in the amount of LV or RV pre-excitation. Computer simulations capture LV and RV behavior during pacing delay variation and may be used in the design of new CRT optimization studies. 


\section{INTRODUCTION}

Cardiac resynchronization therapy (CRT) is an established therapy for heart failure patients with a reduced left ventricular (LV) ejection fraction and left bundle branch block (LBBB) [1]. Through biventricular pacing, CRT aims to establish a more synchronous electrical activation of the ventricles and thereby improves cardiac pump function [2] and clinical outcome [1]. However, approximately one-third of the patients that receive CRT do not benefit from this therapy [3-6].

Programming of both atrioventricular (AV) and ventriculoventricular (VV) pacing delays strongly influences the contractile response to CRT, as determined by both ultrasound and maximum rate of LV pressure rise $\left(\mathrm{LV} d P / \mathrm{dt}_{\max }\right)$ [7-9]. However, meta-analyses of multiple optimization methods showed that pacing delay optimization fails to provide long-term improvement in clinical outcome [7]. Suggested reasons for the absence of long-term benefits of such optimization are that the default "out-of-the-box" delays are already fairly good and that the optimization methods employed are not accurate or robust enough. An alternative explanation could be that most pacing delay optimization strategies that have been developed solely take LV function into account. Right ventricular (RV) function is often overlooked, although several studies show that RV failure is an independent predictor of mortality in patients with LV failure with and without CRT [10,11]. Two clinical studies indicated that there was a poor correlation between the pacing delay settings providing the highest LV dP/dt $t_{\max }$ and $\mathrm{RV} d P / \mathrm{dt}_{\max }$ values $[12,13]$.

In recent years, computational models of cardiac electrophysiology, mechanics of the heart and cardiovascular system have increased our understanding of dyssynchronous heart failure and its treatment with CRT [14]. Right ventricular function and its effect on CRT has however not been studied extensively using a computer modeling approach. Our group has been using the CircAdapt model of the heart and closed-loop circulatory system. While using a simplified cardiac anatomy, the advantages of this model are the inclusion of the entire (systemic and pulmonary) circulation and its high calculation speed (almost real time). In combination with experimental and clinical measurements, CircAdapt has shown to be able to identify and mechanistically understand the electromechanical substrates of the heart that are most responsive to CRT [15-17].

A vast majority of the studies on the heart, also our aforementioned studies, are related largely to the LV. In the present study, we aim to study the changes in both LV and RV contractile response to variations of pacing delay settings in CRT, and to evaluate whether the CircAdapt computer model reliably simulates LV and RV pump function during these interventions. Subsequently we aim to use the computer modeling results to investigate how cardiac output is affected by differences between LV and RV contractile changes. 


\section{METHODS}

\section{Animal experiments}

Animal handling was performed according to the Dutch Law on Animal Experimentation and the European Directive for the Protection of Vertebrate Animals Used for Experimental and Other Scientific Purpose. The protocol was approved by the Animal Experimental Committee of Maastricht University. The animal experimental methodology has been partially published elsewhere [18]. In short, 6 adult mongrel dogs were anesthetized using midazolam $(0,25 \mathrm{mg} / \mathrm{kg} / \mathrm{h})$ and sufentanyl $(3 \mu \mathrm{g} / \mathrm{kg} / \mathrm{h})$ and a complete AV-block was induced by radiofrequency ablation. The dogs received pacing electrodes in the right atrium (A), RV apex and epicardially on the basal posterolateral wall via a left-sided thoracotomy.

Measurements were performed 12-21 weeks after inducing the AV-block using an external custom-built pacing system. RV-only pacing with an A to RV (A-RV) pacing delay of $125 \mathrm{~ms}$ was used as the baseline pacing setting, mimicking the activation pattern as seen in LBBB. The A-RV and A to LV (A-LV) delays were then programmed individually, ranging from 50 to $230 \mathrm{~ms}$ in steps of $20 \mathrm{~ms}$, resulting in 100 possible combinations (Figure 1A). Pacing delay settings were assigned in a random order and baseline recordings were repeated after every $5^{\text {th }}$ setting. During each setting, continuous, invasive hemodynamic and electrocardiographic measurements were performed (Figure 1B). 7F catheter-tip manometers were used for LV and RV pressure measurement. Epicardial electrograms of the LV free wall (LVFW) and RV free wall (RVFW) were recorded from 2 multielectrode custom-made bands holding 102 contact electrodes. Septal endocardial electrograms were recorded from two multielectrode catheters with 7 contact electrodes on the RV septum and 3 on the LV septum. Measurements were recorded for a minimum of two respiratory cycles at each pacing delay setting.

\section{Data analysis}

Local electrical activation times were determined as the duration between the atrial pace and the timepoint of steepest negative deflection of the electrogram using custom software (Figure 1B, green line in local EGM's). For quantification of intraventricular electrical dyssynchrony we calculated total activation time as the difference between earliest and latest activation time of the RVFW (RV TAT) and of the whole LV (LV TAT). The latter was calculated from the combination of epicardial LVFW and endocardial septal electrodes. To quantify electrical interventricular dyssynchrony we used the ventricular electrical uncoupling (VEU) index. VEU was defined as the difference between mean LVFW and mean RVFW activation times [19]. 


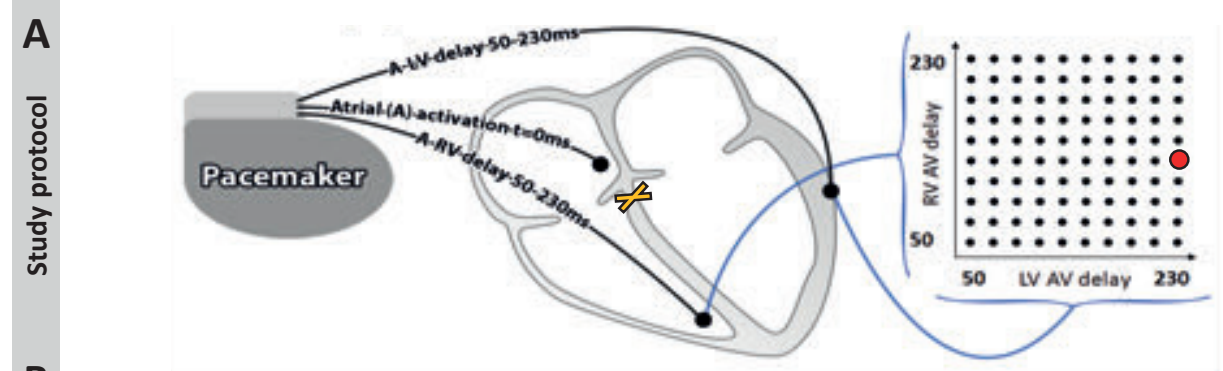

B

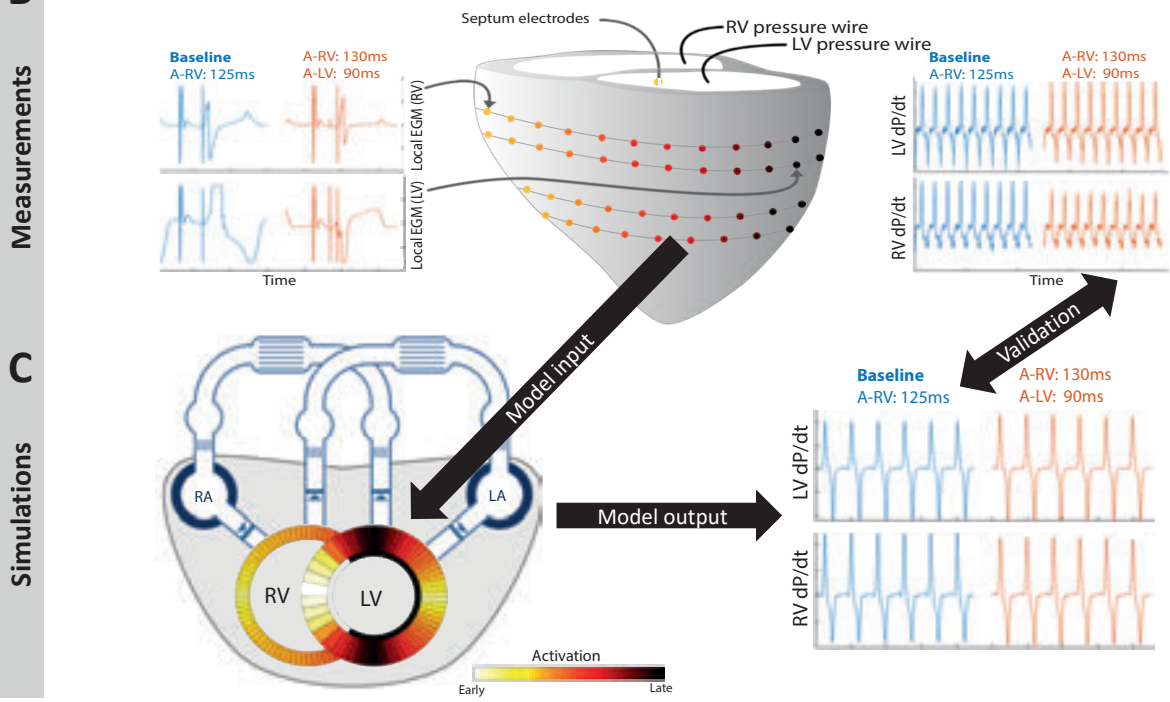

Figure 1. Schematic representation of the methods used in this study. 100 different A-LV/A-RV pacing delay combinations were programmed $(A)$ while pressures and local electrical activation were measured (B). Generic activation maps, derived from local electrograms, were used as onset of mechanical activation in the computer simulations (C). The resulting output of the simulations and measurements was compared for validation purposes. The green square in the heat maps indicates the baseline pacing setting.

For both the LV and the RV, response to pacing was defined as the relative change in $\mathrm{dP}$ / $\mathrm{dt}_{\max }$ compared to the baseline setting. We applied quadratic LOESS fitting to account for measurement variability within each dog [20]. Furthermore, in order to quantify a generic canine response to changes in pacing delay, we created a single representative canine dataset by taking the mean values of the dogs for each setting. We also applied linear $2 \mathrm{D}$ interpolation between the measurements in the heat map visualizations of all pacing delay settings. All these calculations were performed in Matlab 2016A (The Mathworks Inc, Natick MA, USA).

\section{Computer simulations}

The CircAdapt model of the entire heart and circulation [21-23], which can be downloaded from www.circadapt.org, was used to simulate cardiovascular mechanics 
and hemodynamics during pacing delay variations as applied in the animal experiments. Previous experimental and clinical studies have shown that the CircAdapt model realistically relates local ventricular myofiber mechanics to global cardiovascular hemodynamics in dyssynchronous and paced hearts [16,21,24-26].

The source code of the CircAdapt model used for all simulations as well as the Matlab® (The MathWorks, Natick, MA) scripts to perform all simulations and analysis are provided as an online data supplement. In summary, the CircAdapt model is a reduced order model of the human four-chamber heart connected to a closed-loop cardiovascular system, with lumped pulmonary and systemic circulations. It uses a simplified ventricular geometry, where cardiac walls are represented by thick spherical shells consisting of contractile myocardium. The MultiPatch module enables cardiac walls to be subdivided into an arbitrary number of wall segments (patches). Tissue properties and activation time can differ between patches, but all patches within a wall share a common wall tension and curvature. Since wall tension is the same in all patches within a wall, spatial location within a wall is not required to calculate deformation in a patch.

In CircAdapt wall tension and curvature determine cavity pressure through Laplace's law $[21,23]$ Fiber stress in a patch is the sum of an active component, representing myofiber contraction, and a passive component. The active stress component incorporates length-dependence of the force generated and the duration of contraction. The passive component provides a non-linear relationship between myofiber stress and strain. More details on the phenomenological model of myocardial contraction and the validation of the MultiPatch module are previously published by Walmsley et al [21].

\section{Simulating a healthy human reference heart and circulation}

Cardiac adaptation implemented in the CircAdapt model was used to obtain a reference parameterization that represents a healthy human cardiovascular system $[22,27,28]$. The tissue volumes and areas in the cardiac walls and large blood vessels were adapted as described previously (Arts et al., 2005, 2012). A resting cardiac output of 5.1L/min and heart rate of $70 \mathrm{bpm}$ were assumed. Cardiac output was tripled and the heart rate was doubled during the stress-state of the adaptation process. Mean arterial pressure was maintained at $92 \mathrm{mmHg}$ during the adaptation process. The resulting reference simulation was used as the basis for subsequent pacing simulations.

\section{Using electrical activation to simulate the pacing delay optimization protocol}

We divided the ventricular wall in the same amount of segments as the number of available electrodes (52 LV free wall, 50 RV free wall and 10 septal segments). Time of onset of activation was assigned based on the electrical activation times measured in the animal experiments. As previously stated, in the current MultiPatch module the segments were considered to be mechanically coupled in series, meaning that the order in which patches were placed was not significant [21]. This allowed sorting of the activation times per wall 
in each measurement, before taking the median of the dogs, to get a generic activation pattern. The benefit of this generic activation pattern is that it was less affected by differences in band placement, heart size and electrodes with insufficient contact in the dogs.

A representative baseline simulation was obtained by imposing the ventricular activation pattern measured during the experimental baseline condition, i.e. RV-only pacing with an A-RV delay of $125 \mathrm{~ms}$. Systemic vascular resistance was adapted to obtain a mean arterial pressure of $60 \mathrm{mmHg}$ and heart rate was set to $80 \mathrm{bpm}$, both similar to the animal experiments. Furthermore, total circulating blood volume was adjusted so that cardiac output was maintained at $5.1 \mathrm{~L} / \mathrm{min}$. The resulting baseline simulation was used as the starting point for the pacing setting simulations. For each of the 100 pacing delay simulations, the pattern of ventricular activation was changed to the activation pattern measured in the canine experiments and the resulting beat-to-beat changes in ventricular mechanics and hemodynamics were stored until a new hemodynamic steady state was reached. During all pacing simulations, systemic vascular resistance and total circulating blood volume were kept constant in order to quantify the acute effect of pacing-induced changes of ventricular pump mechanics and cardiac hemodynamics. Simulated steadystate $\mathrm{dP} / \mathrm{dt}_{\text {max }}$ values are compared with the experimental measurements. In addition, the simulations extended the animal experiments by providing quantitative insight in the beatto-beat and steady-state changes of ventricular volumes and cardiac output.

\section{RESULTS}

Baseline characteristics for the AV-blocked dogs are described in Table $\mathbf{1 .}$

Table 1. Baseline characteristics in median (range) of dogs during baseline (RV-only, A-RV 125ms pacing)

\begin{tabular}{|c|c|}
\hline & During baseline RV-only pacing \\
\hline Weight (kg) & $19.8(19.4-21.4)$ \\
\hline MAP (mmHg) & $55(42-71)$ \\
\hline Systolic Arterial Pressure $(\mathrm{mmHg})$ & $70(62-81)$ \\
\hline Diastolic Arterial Pressure $(\mathrm{mmHg})$ & $45(31-53)$ \\
\hline $\mathrm{LV} \mathrm{dP/dt}{ }_{\text {max }}(\mathrm{mmHg} / \mathrm{s})$ & $1205(1183-1646)$ \\
\hline 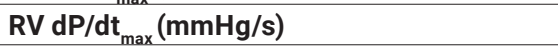 & $520(345-700)$ \\
\hline Weeks between AVB and Sacrifice (weeks) & $13(12-21)$ \\
\hline
\end{tabular}

\section{Electrical effects of altering pacing delay settings}

Figure 2 shows the typical examples of electrical activation patterns acquired using contact mapping in a dog with AV block during LV pre-excitation, simultaneous RV and LV pacing and RV pre-excitation. In case of extreme pre-excitation, capture in the last paced ventricle was lost due to activation via the contralateral ventricle (indicated by the gray line). 
RV pre-excitation led to the largest LV TAT while LV pre-excitation resulted in an increase of RV TAT. During simultaneous pacing, two wave fronts originating from the RV and LV pacing electrodes fused and resynchronized the heart as indicated by a decrease in LV TAT.

LV pre-excitation

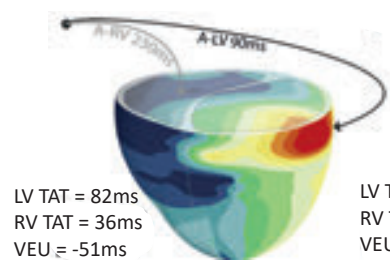

Simultaneous pacing

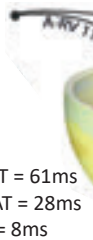

RV pre-excitation

Activation

time

$100 \mathrm{~ms}$

Figure 2. Epicardial electric activation maps in a paced dog heart with complete AV block during LV pre-excitation (left), simultaneous pacing (middle) and RV pre-excitation (right). Black arrows indicate capture, whereas grey arrows indicate loss of capture. TAT: total activation time, VEU: ventricular electrical uncoupling.

Figure 3 shows the changes in electrical dyssynchrony indexes in dogs with variation of pacing delay settings. There was no change in LV and RV TAT and VEU when changing the AV-delay during simultaneous activation of the LV and RV (left column). Both LV and RV TAT (first 2 rows of figure 3 ) were lowest during simultaneous $R V+L V$ pacing. The RV showed the largest TAT during LV pre-excitation or LV-only pacing (upper left corners in the heat maps). LV TAT showed a relatively large increase with large RV pre-excitation (right side of middle panels and lower right corners in heat maps), while RV TAT did not increase much. As indicated by the VEU (bottom row figure 3), during LV-only pacing the LVFW was activated more than $40 \mathrm{~ms}$ before the RVFW. During RV-only pacing the LVFW was, on average, activated more than $20 \mathrm{~ms}$ later than the RVFW. The LVFW and RVFW were activated simultaneously with very slight LV pre-excitation (Figure 3 bottom right, VEU=0).

\section{Hemodynamic effects of altering pacing delay settings}

The changes in LV and $\mathrm{RV} \mathrm{dP} / \mathrm{dt}_{\max }$ in response to changes in pacing delay settings are presented in figure $\mathbf{4}$ (top and bottom, respectively). Increasing AV-delay during simultaneous RV and LV pacing (left column and identity line in the heat maps) hardly affected $\mathrm{LV}$ and $\mathrm{RV} \mathrm{dP} / \mathrm{dt}_{\text {max }}$ in both measurements and simulations. The relative effect of changing VV-delay (green, second column from left) was largest in RV dP/dt ${ }_{\max }$. Changing pacing settings from RV to LV pre-excitation decreased RV dP/dt max $_{\text {ax }}$ with more than $30 \%$ in the experiment. The decrease in $\mathrm{RV} \mathrm{dP} / \mathrm{dt}_{\max }$ was less pronounced in the simulations but followed the same pattern. LV dP/dt $\mathrm{max}_{\text {max }}$ was highest with LV pre-excitation and simultaneous pacing in both the experiment and simulations.

The heat maps of both the animal experiments and computer simulations (right side of figure 4) show a qualitatively similar pattern where the largest changes in both LV and $\mathrm{RV} \mathrm{dP/dt} t_{\max }$ are observed when changing the $\mathrm{VV}$-delay. The largest increase in LV 
$\mathrm{dP} / \mathrm{dt}_{\text {max }}$ in the measurements was reached with a short A-LV (50ms) and A-RV (90ms). $\mathrm{LV}$ pre-excitation led to a larger increase in LV dP/dt $t_{\max }$ than RV pre-excitation during all measurements and simulation, with an optimal LV pre-excitation range of 10-50ms. For RV $\mathrm{dP} / \mathrm{dt}_{\text {max }}$ all LV pre-excitation pacing settings led to a decrease up to 33\% in the experiment and $18 \%$ in the simulations, while RV pre-excitation caused little change compared to baseline (RV-only) pacing.

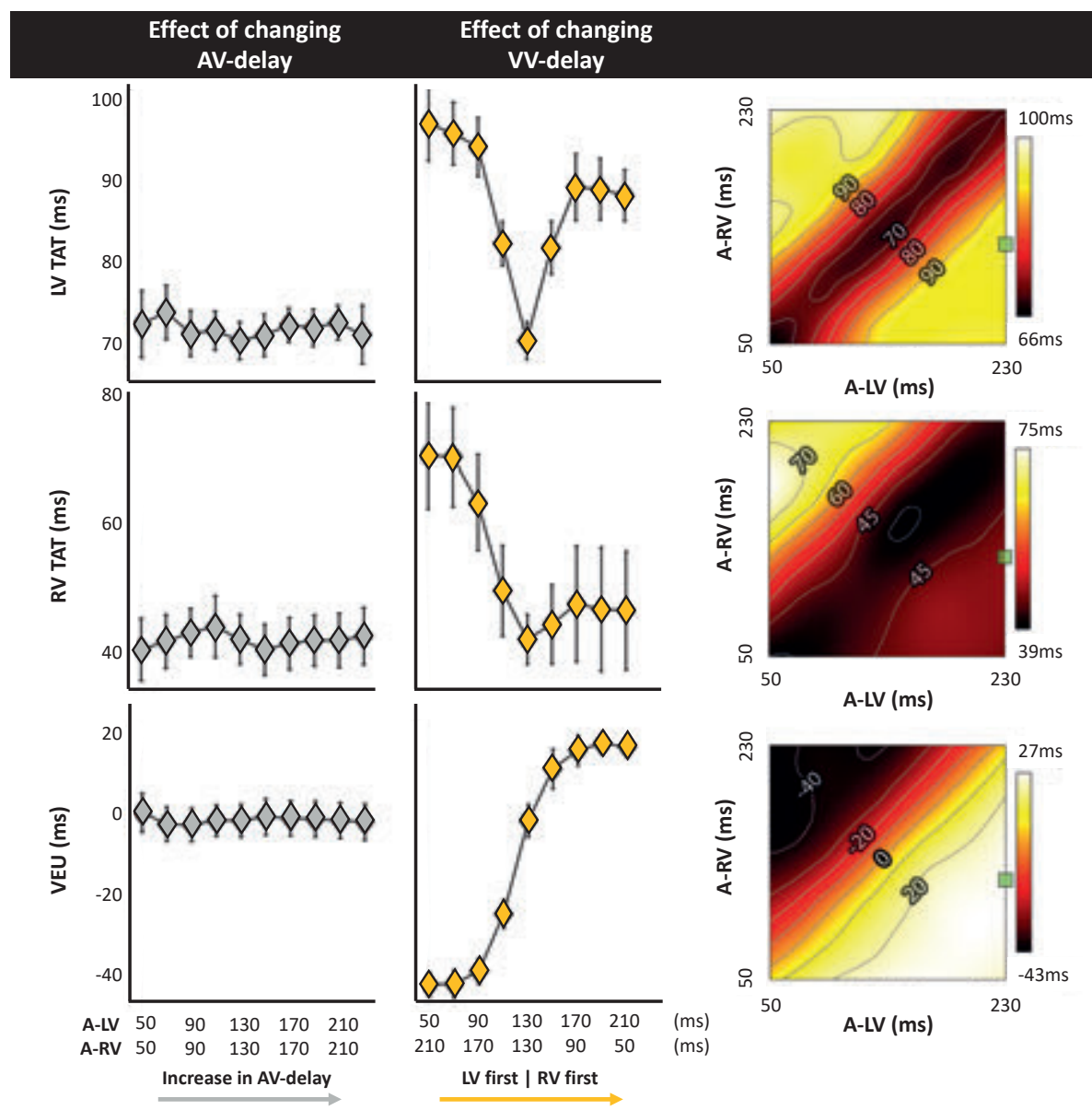

Figure 3. Changes in electrical dyssynchrony indices during variation in pacing delay in the animal experiments. The left column shows the effect of increasing AV-delay during simultaneous RV+LV pacing; the middle column shows changes in VV-delay (yellow, from LV pre-excitation to RV pre-excitation) and the heat maps on the right are the results for all pacing setting (mean of 6 dogs, bars represent standard errors of the mean). From top to bottom: Total activation time (TAT) of the total LV (free wall and septum), RV free wall (RVFW) and VEU (ventricular electrical uncoupling). The green square in each heat map indicate the baseline pacing setting. 


\section{Changes in simulated cardiac output at different pacing delays}

While $\mathrm{dP} / \mathrm{dt}_{\max }$ values are regarded as a measure of ventricular contractility, cardiac output may be more closely related to pump function of the entire heart. Note that due to the closed loop circulation, in a steady state situation cardiac output of the RV and LV are the same. Cardiac output was not determined in the experiments, but it was calculated in the model simulations. In these simulations the changes in cardiac output following a switch in pacing delay differed from the changes in both LV and $\mathrm{RV} \mathrm{dP/dt} t_{\max }$ (Figure 5). Cardiac output was more sensitive to changes in AV-delay than to changes in VV-delay. AV-delays of 50 and $70 \mathrm{~ms}$ led to the largest increases in cardiac output, amounting up to $9 \%$.

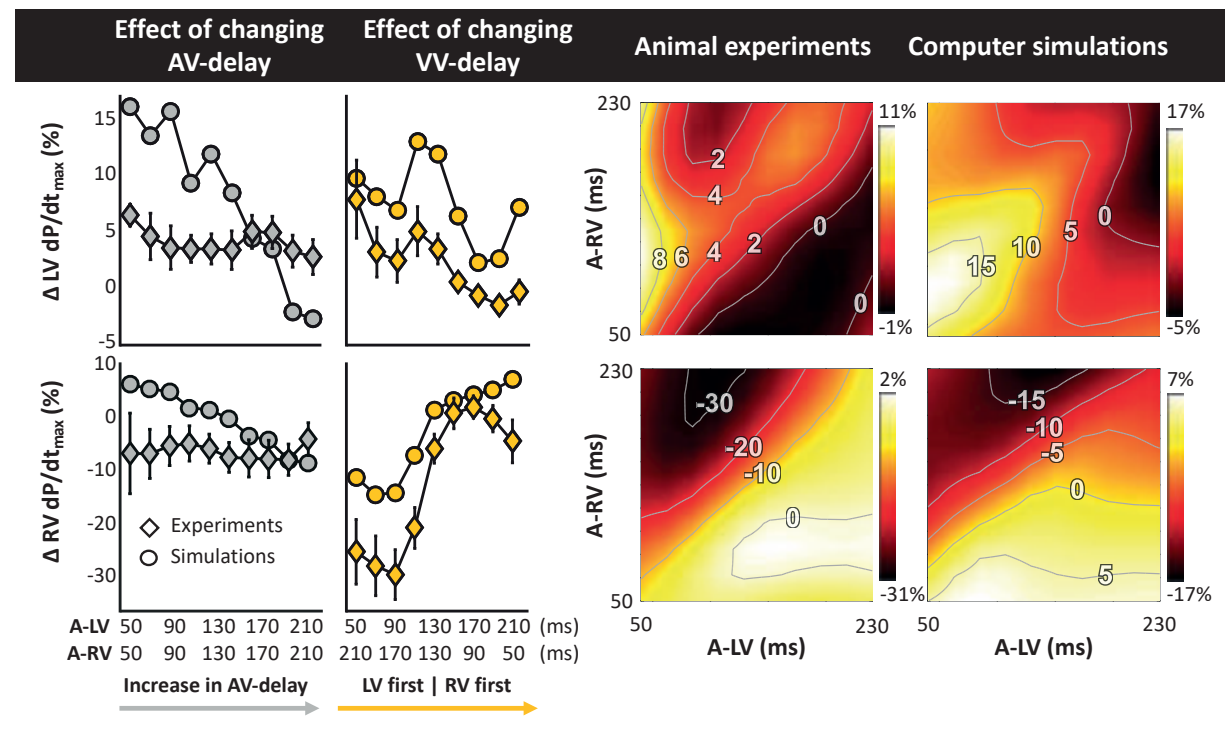

Figure 4. Changes in contractile response as a result of changes in pacing delays in experiments

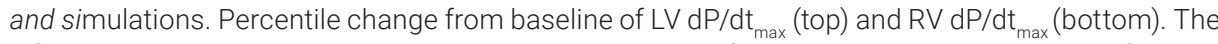
left rows depict the same $A V$ and $V V$-delay settings as in figure $\mathbf{3}$ are shown. Heat maps for both the experiment ( $3^{\text {rd }}$ column) and simulations ( $4^{\text {th }}$ column). Diamonds: Canine measurements (Mean (standard error of the mean) of 6 dogs; Circles: Simulation output.

In order to find an explanation for the differences in behavior between cardiac output and $\mathrm{RV}$ and $\mathrm{LV} \mathrm{dP/dt} t_{\max }$ we compared the time course of these parameters as well as end diastolic volume during the first beats after start of a certain setting, in this case LV preexcitation (Figure 6). In the first beat after the change in pacing setting and therefore also activation sequence (see above), both LV stroke volume and $\mathrm{dP} / \mathrm{dt}_{\max }$ increased while RV stroke volume and $\mathrm{dP} / \mathrm{dt}_{\max }$ decreased. In the subsequent beat RV end diastolic volume increased, due to the smaller stroke volume of the previous beat, whereas LV end diastolic volume decreased. As a consequence of these end diastolic volume changes, RV stroke volume recovered and LV stroke volume decreased to some extent and in the third and subsequent beats a steady state (SS) was reached, with stroke volume in both ventricles (and therefore cardiac output) increasing by about 3\%. This example, representative for 
the other conditions, illustrates that $\mathrm{dP} / \mathrm{dt}_{\max }$ was largely independent of preload, whereas stroke volume depended on it, most likely due to the length dependent activation, implemented in the CircAdapt model (see methods).

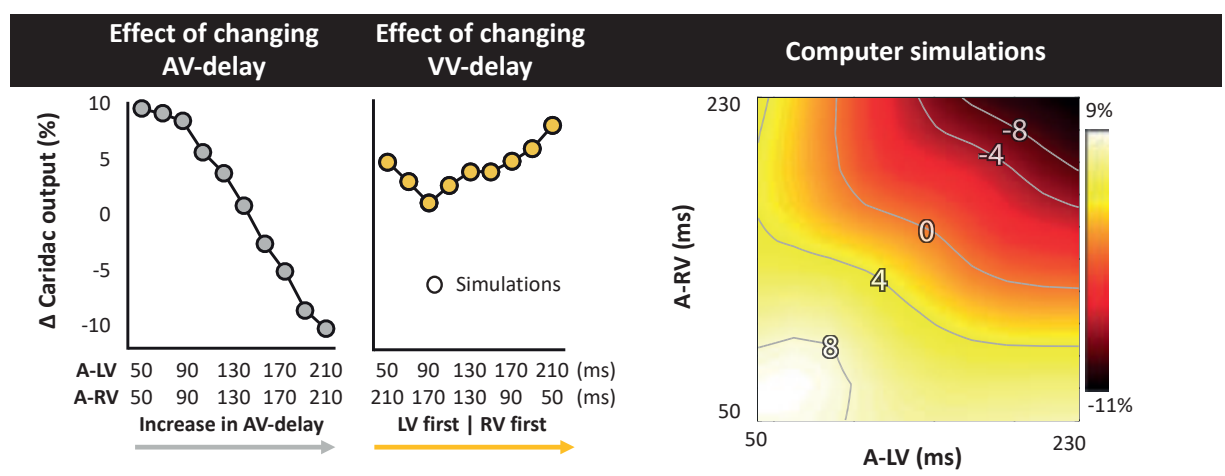

Figure 5. Relative change in simulated steady-state cardiac output with a change in pacing delay settings. Depicted are the changes relative to baseline (see text).

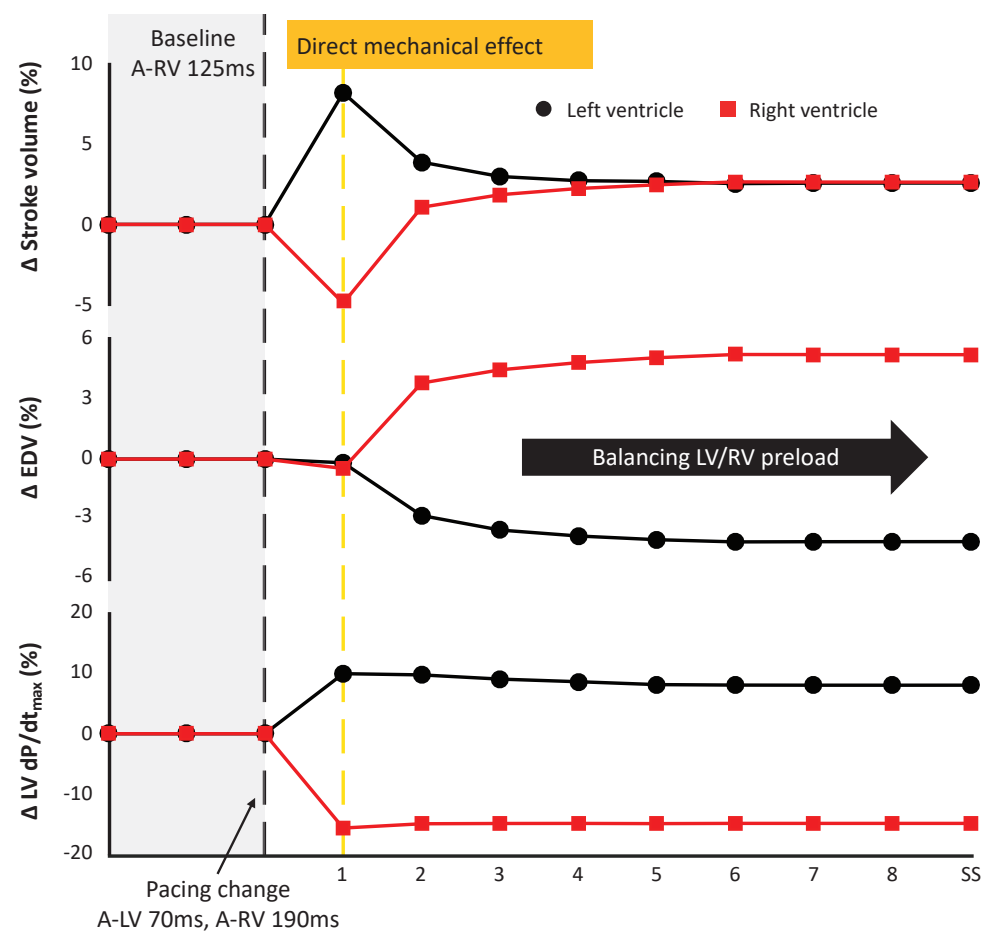

Figure 6. Time courses of the relative change of stroke volume (top), end-diastolic volume (EDV, $\mathrm{mid}$ ) and $\mathrm{dP} / \mathrm{dt}_{\max }$ of the LV (black circles) and RV (red squares) after changing pacing delay from baseline (A-RV 125ms) to LV pre-excitation (A-LV 70ms, A-RV 190ms) in computer simulations. The black dashed line indicates the start of the change in pacing delay. The numbers on the horizontal axis indicate the number of simulated cardiac cycles. SS: steady state. 
Figure 7 shows the response of stroke volume of the LV and RV after simulated programming of nine different pacing settings. In the first beat RV stroke volume remained either unchanged or decreased as compared to baseline, indicating little direct mechanical benefit of the change in pacing delay for the RV. However, similar to the example in figure 6, copied into the left upper panel of figure 7, RV stroke volume changed considerably in subsequent beats. While changes in LV stroke volume initially differed from RV stroke volume, a steady state was reached after several simulated beats. Note that the largest benefit in stroke volume, and therefore cardiac output, was primarily dependent on AVdelay. For example, in the bottom row (A-RV 70ms) the optimized RV filling improved stroke volume to such a degree that, through the serial coupling, LV preload increased, leading to a further increase in LV stroke volume after the second cycle. On the other hand, at longer AV-delays this atrial-ventricular coupling decreases, resulting in a lower steadystate cardiac output.

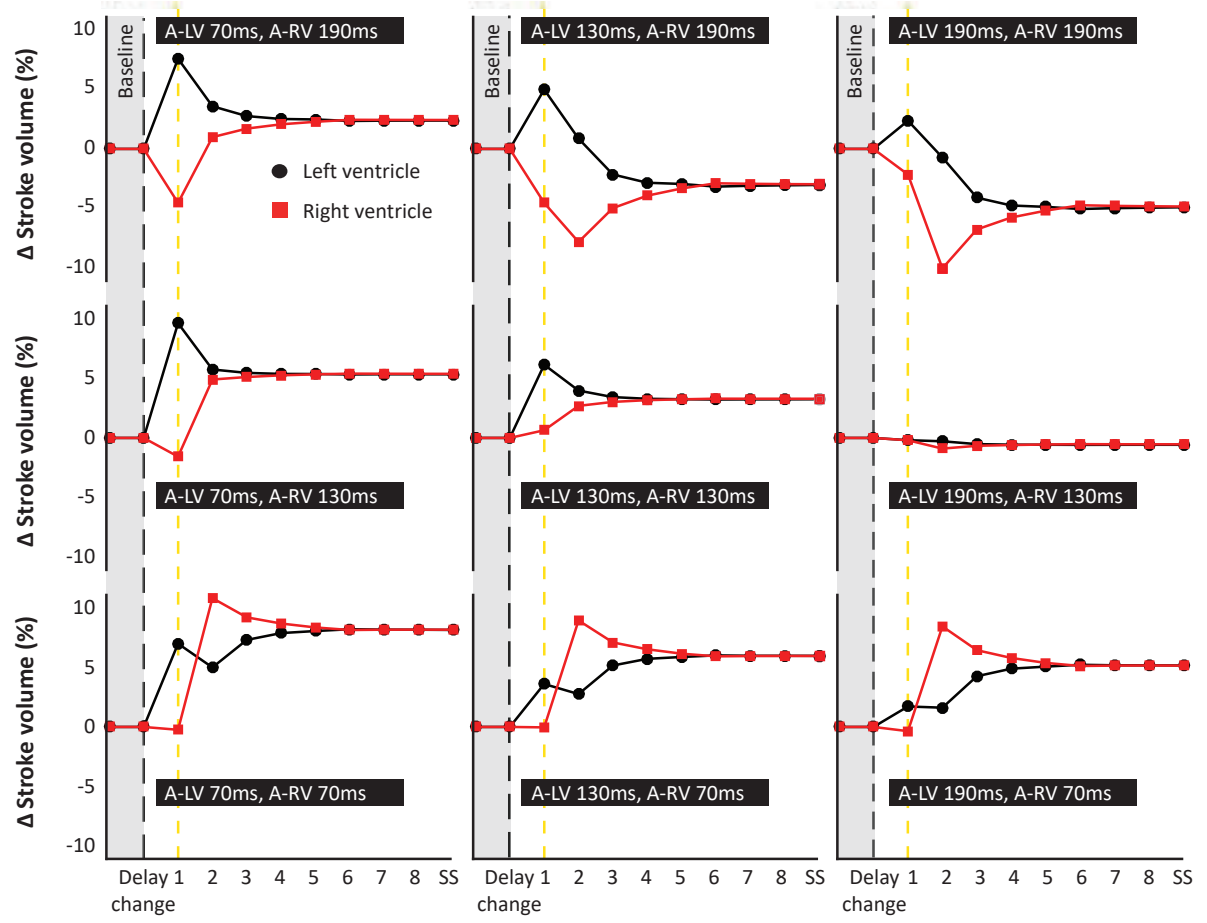

Figure 7. Relative change in stroke volume of the left (black circles) and right (red squares) ventricle over a number of simulated cycles until steady-state (SS) after changing pacing delay (the 9 settings shown in the black bars). Black dashed line indicates the moment of changing the pacing delays while the yellow line marks the first beat. 


\section{DISCUSSION}

In this study we investigated the influence of LV and RV pacing delay settings on LV and $\mathrm{RV}$ electrical activation and contractility in animal studies and computer simulations. Both studies showed that LV TAT is smallest during synchronous RV and LV stimulation and increases when VV-delays increase. RV TAT becomes larger in particular during LV preexcitation. LV and RV contractility vary most, and in opposite direction, with changes in VV-delay settings. After demonstrating the realistic simulations in the model, we used the model to calculate cardiac output changes and to explain why changes cardiac output differed from both RV and LV contractility. The latter findings demonstrate how a model like CircAdapt can extend mechanistic understanding of circulatory changes due to a device therapy.

\section{LV and RV contractility respond in opposite manner to variations in VV pacing delays}

A key finding in the present study is that LV and RV dP/dt $t_{\max }$ change in opposite direction when changing the $\mathrm{V} V$ pacing delay. Sciaraffia et al previously demonstrated that RV and LV $\mathrm{dP} / \mathrm{dt}_{\max }$ identify different 'optimal' $\mathrm{V} \mathrm{V}$-delays in most of the patients included in their study [12]. Furthermore, Houston et al recently showed that in patients with dyssynchronous heart failure, RV-only pacing leads to higher RV dP/dt max $_{\text {ax }}$ than LV-only or simultaneous LV+RV pacing [29]. In an experimental study in pigs, different $V V$-delays were tested at different pacing locations [30]. Similar to our study, these investigators found that RV pre-excitation led to a higher RV $\mathrm{dP} / \mathrm{dt}_{\max }$ than LV pre-excitation. The fact that findings were consistent in patients, animals and a computer model implies that the opposing changes in hemodynamics, caused by varying $\mathrm{V} V$ pacing delays, are caused by a universal mechanism.

Another key finding is that ventricular specific pre-excitation is required for a good contractile function in both the LV and RV. This is illustrated by LV pre-excitation increasing $\mathrm{LV} d P / \mathrm{dt}_{\max }$ and RV pre-excitation leading to the largest RV dP/dt ${ }_{\max }$ values. In contrast, changes in AV-delay have less effect on measured and simulated LV and RV dP/dt ${ }_{\max }$. In a previous study, in which we evaluated the relative importance of interventricular and intraventricular dyssynchrony for contractile response to CRT (change in LV dP/dt ${ }_{\text {max }}$ ), it was demonstrated that interventricular dyssynchrony during intrinsic rhythm is the dominant electrical substrate driving response to CRT [15]. In contrast, intraventricular dyssynchrony showed little effect on LV dP/dt max' which is in line with experimental observations that

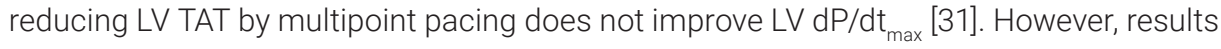
in this study suggest that intraventricular dyssynchrony might still play a modulating role since increase in LV TAT, with large LV pre-excitation, led to a decrease in LV dP/ $\mathrm{dt}_{\max }$ compared to slight LV pre-excitation. Because RV TAT increases concurrently with decreases in VEU we cannot distinguish if either intra- or interventricular dyssynchrony has a larger effect on RV contractility. 
In agreement with our observations, other studies in patients have shown that the lowest electrical dyssynchrony does not necessarily lead to the highest LV contractility or better clinical outcome [17,32]. Optimization based on minimization of electrical dyssynchrony alone might therefore not be sufficient. Even if the optimal electrical activation for LV contractility is known, further clinical studies are required to investigate whether the gain in LV contractility outweighs the loss of RV contractility, especially since cardiac output might not match either or both but be a combination/compromise.

\section{CircAdapt simulations capture both LV and RV contractile response to pacing delay changes}

The present study demonstrates that the CircAdapt model can capture pacing-induced changes to both LV and RV contractile function. It is especially the response of the RV to pacing that has been less well studied, both by our group and by others.

The use of experimental measurements electrical activation of the paced dog heart, derived from canine experiments, coupled to the simulation of the entire circulation, resulted in changes in $\mathrm{RV}$ and $\mathrm{LV} \mathrm{dP} / \mathrm{dt}_{\text {max }}$ that closely mimicked values measured in dog experiments.

Earlier studies have shown that CircAdapt enables realistic simulation of cardiac response to CRT, mostly focusing on LV function [15-17]. In one of those studies, it was demonstrated that the RV plays an important role in the improvement of LV function during LV-only pacing [17]. The present study extends the mechanistic insight in the working action of CRT in the context of pacing delay optimization, where the complex mechanical and hemodynamic interactions between the four cardiac cavities and the surrounding circulations are found to be important.

As demonstrated in this study, the CircAdapt model can capture the complexity of different LV and RV responses to CRT by incorporating several relevant components of cardiovascular interaction. Firstly, it realistically incorporates direct mechanical interaction between the three ventricular walls [23]. Secondly, it allows realistic simulation of regional myocardial mechanics in the ventricular walls of the asynchronously activated heart $[21,26]$, thereby enabling experimentally measured activation times to be imposed and the related intraventricular heterogeneities in mechanical myofiber behavior to be simulated. Thirdly, it is a closed-loop system allowing for indirect (serial) hemodynamic interaction between the left and right side of the heart through the systemic and pulmonary circulations [22,33]. Fourthly, its four-chamber heart captures the dynamics of hemodynamic atrioventricular interactions [34]. Lastly, it includes the mechanical interaction through pericardial constraint, with an increase in the volume of a chamber altering the pressure in the other chambers and, hence, diastolic filling and septal position [35]. 


\section{Simulation-derived mechanistic insights}

The ability of CircAdapt to realistically simulate both LV and RV hemodynamics during pacing allowed us to further study the impact of differences between both chambers on cardiac output. In particular, the model showed a difference in response between $\mathrm{dP} / \mathrm{dt}_{\max }$ and stroke volume/cardiac output. Similar differences have been observed in a clinical study where LV dP/dt $\mathrm{dmax}_{\text {ax }}$ responses differed from responses in stroke work [36].

Our simulations provided a plausible explanation for this paradoxical observation. While LV and RV stroke volume of the first beat after a change of pacing delay can differ substantially, a common steady-state stroke volume and thereby cardiac output is reached due to balancing ventricular preload conditions during the next few beats. There are three main determinants of the newly achieved steady-state preload condition. First, the changes in stroke volumes directly change the end-systolic volumes and thereby the end-diastolic volumes in the next beat. Second, the pacing-induced changes in effective left and right AV-delays change the efficiency of atrial and ventricular diastolic filling and subsequent systolic contraction. Thirdly, changes in stroke volume affect the filling of the other ventricle through the systemic and pulmonary circulations.

While $\mathrm{dP} / \mathrm{dt}_{\max }$ changes congruently with first-beat stroke volume in the same ventricle, it is less affected by changes in preload. As a result, LV and RV dP/dt $t_{\text {max }}$ are much more sensitive to changes of $\mathrm{VV}$-delay and, hence, asynchrony of electrical activation than to changes of AV-delay. On the other hand, changes of AV-delay affect cardiac output more than $L V$ and $R V d P / \mathrm{dt}_{\text {max }}$ :

\section{Computer modeling in therapy optimization}

Other cardiac computer modelling studies have been conducted to investigate other factors in the optimization of CRT therapy. For example, in a cohort of 648 virtual patients it was found that the location of the LV pacing site is an important factor in response to CRT [37]. Electrophysical cardiac computer modeling studies also demonstrated the importance of $L V$ pacing site and the potential of simulations to predict the electrical optimal pacing location and setting [38-40]. Our study shows, however, that an electrical optimum (lowest electrical dyssynchrony) might not necessarily be optimal for overall pump function.

The CircAdapt simulations performed for this study can run on a single core in real time. CircAdapt requires activation time as input and lacks the cardiac electrophysiological model necessary to extract this information from standard clinical data. This input could, however, potentially be generated by other models, for example the ones referred to in the previous paragraph. This would also allow for testing of alternative pacing sites, which would result in different activation patterns, which subsequently can be used as input for CircAdapt simulations. A workflow where fast and anatomically realistic cardiac electrophysiological simulations are combined with cardiac mechanical and circulatory CircAdapt simulations might further increase the clinical applicability of cardiac computer models. 


\section{Study Limitations}

In this study the pacing experiments were performed in relatively healthy canine hearts. Previous work from our group showed that chronic total AV-block leads to structural changes (hypertrophy) and electrical remodeling (QT-time prolongation), but normal contractility [41]. On the other hand, patients treated with CRT have different levels of myocardial remodeling and heart failure, which may affect the response to pacing [42]. Results of this study might differ from patient data since long-term structural remodeling was not included in both the animal and computational experiments. On the other hand, differences in RV and LV response observed in this study could mean that different pacing settings might affect the positive and/or negative remodeling of the LV and RV.

A major difference between this animal experiment and patients in day-to-day life is that the animals were anesthetized. To allow comparison between the experiments and simulations, the model's LV afterload in the baseline situation was adapted to fit the measured mean arterial pressure in the dogs. After the change in activation delays regulation was disabled, which is likely similar to the anesthetic condition were regulation is slow. Caution should however be taken when translating results of this study towards the clinical setting considering that loading conditions potentially affect the effect of pacing delay changes [43]. Future studies are needed to investigate how load-dependent the observed effects of pacing delay optimization are and how homeostatic regulation interacts with changes in LV and RV contractility. A final limitation of the CircAdapt (and most other computer models in this field) is that changes in function of the autonomic nervous system are not taken into account.

\section{Clinical perspective}

The results of this study raise the question what outcome measure is best to use for optimization of pacing delay. Current clinical practice focusses almost exclusively on the LV, using parameters like LV dP/dt max aortic outflow integral, and LV systolic (or aortic) pressure. This study demonstrates that improving LV function can reduce RV function. Furthermore, cardiac output is not necessarily increasing when LV dP/dt max $_{\text {increases. }}$ incer Hence, an exclusive focus on the LV might not lead to the best overall outcome. Therefore, future studies on optimization of therapy should not exclusively focus on the LV but also include measures of RV and/or whole heart function. In our analysis of cardiac output we also demonstrated that the moment of measurement affects what physiological phenomenon is actually observed. Contractile function alone might better be observed in the first beats while longer lasting measurements, that allow reaching a steady state, will provide more information about the loading of the heart and its interaction with homeostatic regulation. Insights acquired in the present opens the way for designing better optimization protocols, possibly even including computer modeling. 


\section{CONCLUSION}

The LV and the RV respond in an opposite manner to LV or RV pre-excitation. LV preexcitation improved LV contractility and decreased RV contractility, while RV pre-excitation had the opposite effects. The CircAdapt computer model realistically captures these opposite responses of LV and RV contractile function. Computer simulations extend animal experimental findings by revealing that improving ventricular contractility does not necessarily lead to an improvement of cardiac output. This study demonstrates the potential of CircAdapt to provide a valuable and efficient in silico platform for further optimization studies for device therapy. 


\section{REFERENCES}

1 Brignole M, Auricchio A, Baron-Esquivias G, Bordachar P, Boriani G, Breithardt O-A, et al. 2013 ESC Guidelines on cardiac pacing and cardiac resynchronization therapy: the Task Force on cardiac pacing and resynchronization therapy of the European Society of Cardiology (ESC). Developed in collaboration with the European Heart Rhythm Association. Eur Heart J. 2013;34(29):2281-329.

2 Vernooy K, Cornelussen RN, Verbeek XA, Vanagt WY, van Hunnik A, Kuiper M, et al. Cardiac resynchronization therapy cures dyssynchronopathy in canine left bundle-branch block hearts. Eur Heart J. 2007;28(17):2148-55.

3 Prinzen FW, Vernooy K, Auricchio A. Cardiac resynchronization therapy: state-of-the-art of current applications, guidelines, ongoing trials, and areas of controversy. Circulation. 2013;128(22):2407-18.

4 Auricchio A, Prinzen FW. Non-Responders to Cardiac Resynchronization Therapy. Circ J. 2011;75(3):521-7.

5 Cleland JG, Daubert JC, Erdmann E, Freemantle N, Gras D, Kappenberger L, et al. The effect of cardiac resynchronization on morbidity and mortality in heart failure. $\mathrm{N}$ Engl $\mathrm{J}$ Med. 2005;352:1539-49.

6 Abraham WT, Fisher WG, Smith AL, Delurgio DB, Leon AR, Loh E, et al. Cardiac resynchronization in chronic heart failure. N Engl J Med. 2002;346(24):1845-53.

7 Auger D, Hoke U, Bax JJ, Boersma E, Delgado V. Effect of atrioventricular and ventriculoventricular delay optimization on clinical and echocardiographic outcomes of patients treated with cardiac resynchronization therapy: A meta-analysis. Am Heart J. 2013;166(1):20-9.

8 Strik M, van Middendorp LB, Houthuizen P, Ploux S, van Hunnik A, Kuiper M, et al. Interplay of electrical wavefronts as determinant of the response to cardiac resynchronization therapy in dyssynchronous canine hearts. Circ Arrhythm Electrophysiol. 2013;6(5):924-31.

9 Bogaard MD, Meine M, Tuinenburg AE, Maskara B, Loh P, Doevendans PA. Cardiac resynchronization therapy beyond nominal settings: Who needs individual programming of the atrioventricular and interventricular delay? Europace. 2012;14(12):1746-53.

10 Ricci F, Mele D, Bianco F, Bucciarelli V, De Caterina R, Gallina S. Right heart-pulmonary circulation unit and cardiac resynchronization therapy. Am Heart J. 2017;185:1-16.

11 Groote PDE, Millaire A, Foucher-hossein C, de Groote P, Millaire A, Foucher-hossein C, et al. Right ventricular ejection fraction is an independent predictor of survival in patients with moderate heart failure. J Am Coll Cardiol. 1998;32(4):948-54.

12 Sciaraffia E, Malmborg H, Lönnerholm S, Blomström P, Blomström Lundqvist C. Right ventricular contractility as a measure of optimal interventricular pacing setting in cardiac resynchronization therapy. Europace. 2009;11(11):1496-500.

13 Hyde ER, Behar JM, Crozier A, Claridge S, Jackson T, Sohal M, et al. Improvement of Right Ventricular Hemodynamics With Left Ventricular Endocardial Pacing During Cardiac Resynchronization Therapy. Pacing Clin Electrophysiol. 2016;

14 Lee AWC, Costa CM, Strocchi M, Rinaldi CA, Niederer SA. Computational Modeling for Cardiac Resynchronization Therapy. J Cardiovasc Transl Res. 2018;11(2):92-108.

15 Huntjens PR, Ploux S, Strik M, Walmsley J, Ritter P, Haissaguerre M, et al. Electrical Substrates Driving Response to Cardiac Resynchronization Therapy. Circ Arrhythmia Electrophysiol. 2018;11(4):e005647.

16 Lumens J, Tayal B, Walmsley J, Delgado-Montero A, Huntjens PR, Schwartzman D, et al. Differentiating Electromechanical From Non-Electrical Substrates of Mechanical Discoordination to Identify Responders to Cardiac Resynchronization Therapy. Circ Cardiovasc Imaging. 2015;8(9):e003744. 
17 Lumens J, Ploux S, Strik M, Gorcsan 3rd J, Cochet H, Derval N, et al. Comparative electromechanical and hemodynamic effects of left ventricular and biventricular pacing in dyssynchronous heart failure: electrical resynchronization versus left-right ventricular interaction. J Am Coll Cardiol. 2013;62(25):2395-403.

18 Strik M, van Deursen CJ, van Middendorp LB, van Hunnik A, Kuiper M, Auricchio A, et al. Transseptal conduction as an important determinant for cardiac resynchronization therapy, as revealed by extensive electrical mapping in the dyssynchronous canine heart. Circ Arrhythm Electrophysiol. 2013;6(4):682-9.

19 Ploux S, Lumens J, Whinnett Z, Montaudon M, Strom M, Ramanathan C, et al. Noninvasive electrocardiographic mapping to improve patient selection for cardiac resynchronization therapy: beyond QRS duration and left bundle branch block morphology. J Am Coll Cardiol. 2013;61(24):2435-43.

20 Cleveland WS. Robust locally weighted regression and smoothing scatterplots. J Am Stat Assoc. 1979;74(368):829-36.

21 Walmsley J, Arts T, Derval N, Bordachar P, Cochet H, Ploux S, et al. Fast Simulation of Mechanical Heterogeneity in the Electrically Asynchronous Heart Using the MultiPatch Module. PLoS Comput Biol. 2015;11(7).

22 Arts T, Delhaas T, Bovendeerd P, Verbeek X, Prinzen F. Adaptation to mechanical load determines shape and properties of heart and circulation: the CircAdapt model. Am J Physiol Heart Circ Physiol. 2005;288:1943-54.

23 Lumens J, Delhaas T, Kirn B, Arts T. Three-wall segment (TriSeg) model describing mechanics and hemodynamics of ventricular interaction. Ann Biomed Eng. 2009/09/01. 2009;37(11):223455 .

24 Huntjens PR, Walmsley J, Ploux S, Bordachar P, Prinzen FW, Delhaas T, et al. Influence of left ventricular lead position relative to scar location on response to cardiac resynchronization therapy: a model study. Eur Eur Pacing, Arrhythmias, Card Electrophysiol. 2014;16 Suppl 4:iv628.

25 Walmsley J, Huntjens PR, Prinzen FW, Delhaas T, Lumens J. Septal flash and septal rebound stretch have different underlying mechanisms. Am J Physiol Heart Circ Physiol. 2015;(23):ajpheart.00639.2015.

26 Leenders GE, Lumens J, Cramer MJ, De Boeck BWL, Doevendans PA, Delhaas T, et al. Septal deformation patterns delineate mechanical dyssynchrony and regional differences in contractility: analysis of patient data using a computer model. Circ Hear Fail. 2012;5:87-96.

27 Arts T, Prinzen FW, Snoeckx LHEH, Rijcken JM, Reneman RS. Adaption of cardiac structure by mechanical feedback in the environment of the cell: A model study. Biophys J. 1994;66(4):95361.

28 Arts T, Lumens J, Kroon W, Delhaas T. Control of whole heart geometry by intramyocardial mechano-feedback: A model study. PLoS Comput Biol. 2012;8(2).

29 Houston BA, Sturdivant JL, Yu Y, Gold MR. Acute biventricular hemodynamic effects of cardiac resynchronization therapy in right bundle branch block. Hear Rhythm. 2018;15(10):1525-32.

30 Quinn TA, Berberian G, Cabreriza SE, Maskin LJ, Weinberg AD, Holmes JW, et al. Effects of sequential biventricular pacing during acute right ventricular pressure overload. Am J Physiol - Hear Circ Physiol. 2006;291(5):H2380-7.

31 Ploux S, Strik M, van Hunnik A, van Middendorp L, Kuiper M, Prinzen FW. Acute electrical and hemodynamic effects of multisite left ventricular pacing for cardiac resynchronization therapy in the dyssynchronous canine heart. Hear Rhythm. 2014;11(1):119-25.

32 Thibault B, Ducharme A, Harel F, White M, Omeara E, Guertin MC, et al. Left ventricular versus simultaneous biventricular pacing in patients with heart failure and a grs complex ???120 milliseconds. Circulation. 2011;124(25):2874-81.

33 Lumens J, Blanchard DG, Arts T, Mahmud E, Delhaas T. Left ventricular underfilling and not septal bulging dominates abnormal left ventricular filling hemodynamics in chronic thromboembolic pulmonary hypertension. AJP Hear Circ Physiol. 2010;299(4):H1083-91. 
34 Jones S, Lumens J, Sohaib SMA, Finegold JA, Kanagaratnam P, Tanner M, et al. Cardiac resynchronization therapy: mechanisms of action and scope for further improvement in cardiac function. Eur Eur Pacing, Arrhythmias, Card Electrophysiol. 2016/07/15. 2017;19(7):1178-86.

35 Palau-Caballero G, Walmsley J, Van Empel V, Lumens J, Delhaas T. Why septal motion is a marker of right ventricular failure in pulmonary arterial hypertension: mechanistic analysis using a computer model. Am J Physiol - Hear Circ Physiol. 2017;312(4):H691-700.

36 van Everdingen WM, Zweerink A, Salden OAE, Cramer MJ, Doevendans PA, van Rossum AC, et al. Atrioventricular optimization in cardiac resynchronization therapy with quadripolar leads: should we optimize every pacing configuration including multi-point pacing? EP Eur. 2018;(July):1-9.

37 Crozier A, Blazevic B, Lamata P, Plank G, Ginks M, Duckett S, et al. The relative role of patient physiology and device optimisation in cardiac resynchronisation therapy: A computational modelling study. J Mol Cell Cardiol. 2016;96:93-100.

38 Reumann M, Farina D, Miri R, Lurz S, Osswald B, Dössel O. Computer model for the optimization of AV and VV delay in cardiac resynchronization therapy. Med Biol Eng Comput. 2007;45(9):84554.

39 Miri R, Graf IM, DÖssel O. Efficiency of timing delays and electrode positions in optimization of biventricular pacing: A simulation study. IEEE Trans Biomed Eng. 2009;56(11):2573-82.

40 Niederer S, Mitchell L, Smith N, Plank G. Simulating human cardiac electrophysiology on clinical time-scales. Front Physiol. 2011;APR(April).

41 Peschar M, Vernooy K, Vanagt WYR, Reneman RS, Vos MA, Prinzen FW. Absence of reverse electrical remodeling during regression of volume overload hypertrophy in canine ventricles. Cardiovasc Res. 2003;58(3):510-7.

42 Nguyên UC, Verzaal NJ, van Nieuwenhoven FA, Vernooy K, Prinzen FW. Pathobiology of cardiac dyssynchrony and resynchronization therapy. EP Eur. 2018;6(11):1660-5.

43 Quinn TA, Cabreriza SE, Richmond ME, Weinberg AD, Holmes JW, Spotnitz HM. Simultaneous variation of ventricular pacing site and timing with biventricular pacing in acute ventricular failure improves function by interventricular assist. Am J Physiol Heart Circ Physiol. 2009;297(6):H2220-6. 





\section{GENERAL DISCUSSION}

The review of the literature on cardiac resynchronization therapy (CRT) in chapter 2 describes what is known about the electrophysiological and hemodynamic changes that occur during CRT in both animals and patients. It starts by explaining how left bundle branch block (LBBB) induced dyssynchronous contraction of the heart results in impaired cardiac function because of an increase in wasted work. This also explains how CRT is able to electromechanically resynchronize the contraction of the ventricles and improve pump function. At least two factors are important for achieving the best possible benefit of CRT: the presence of clear dyssynchrony (like LBBB) and the use of optimal timing between atrial and ventricular stimulation.

This thesis investigated 1) whether CRT may be able to improve ventricular filling and cardiac function in hearts with prolonged atrioventricular delay (first degree AV-block) in the absence of ventricular dyssynchrony (chapters $\mathbf{3}$ and chapter $\mathbf{4}$ ) and 2) novel ways to optimize pacemaker settings in order to create the best possible ventricular filling as well as ventricular resynchronization (chapters $\mathbf{5}$ and chapter $\mathbf{6}$ ).

Where CRT is currently only indicated for heart failure patients with ventricular dyssynchrony (preferably LBBB), the results presented in chapter $\mathbf{3}$ provide strong evidence that restoring proper AV-coupling in first degree AV-block can improve hemodynamic function. In heart failure patients and animals optimizing the AV-delay using biventricular (BiV) pacing resulted in an increase in mean arterial pressure and stroke volume. This was achieved by an improvement in ventricular filling. Optimization of timing of passive (E) and active (A) ventricular filling resulted in an increase of forward flow over the mitral valve and a decrease in diastolic mitral regurgitation (MR). Computer simulations using the CircAdapt model were used to further unravel the influence of ventricular filling on the hemodynamic outcome. Furthermore, we showed that right ventricular (RV) pacing-induced dyssynchrony abrogates the hemodynamic benefits of better AV-coupling and should therefore be avoided. In conclusion, BiV pacing might be therapeutic option in patients with heart failure and prolonged PR-interval that is currently not part of the guidelines.

Chapter $\mathbf{4}$ uses the porcine model of total AV-block to investigate the interplay between interatrial delay (IAD, dependent on among other atrial sensing or atrial pacing) and interventricular dyssynchrony (IVD, influenced by ventricular pacing site) on filling of both ventricles. While commonly AV-delay is only considered for the LV, the serial coupling between the pulmonary and systemic circulation makes it likely that proper filling of both ventricles is important. The results in this study clearly show that the optimal AV-delay, quantified as the AV-delay with the highest relative increase in cardiac output, differs between the left side and right side of the heart. We propose the mean effective AV-delay as an alternative parameter, that is based on timings of mechanical activation of all four heart chambers, to predict optimal BiV function. We showed that this parameter can estimate 
the optimal AV-delay in a manner that is relatively independent of IAD and IVD, especially when corrected for the heart rate. Therefore, the mean effective AV-delay may be a useful measure for pacemaker optimization in the future.

After showing the importance of an optimal AV-delay in chapter $\mathbf{3}$ and chapter $\mathbf{4}$, we focused on how to optimize the optimal AV-delay for CRT using intracardiac derived accelerometer signals and in particular the first heart sound (SonR1). In chapter $\mathbf{5}$ we showed that the SonR1 amplitude strongly correlates with LV and RV contractility in a canine LBBB model. In animals that responded successfully to CRT, the SonR1 signal could be used to predict the LV dP/dt max -based optimal AV-delay. This study showed that the SonR1 amplitude is influenced by changes in cardiac contractility and time interval between atrial and ventricular contraction, while interventricular dyssynchrony plays a minor role. Placement of the SonR sensor in the RA seems to assess cardiac function better than an RV-placed sensor.

Finally, in chapter 6 we combined data from a canine LBBB model with computer simulations to show that the LV and RV respond in an opposite manner to variations in the amount of LV or RV pre-excitation. Pre-exciting the LV leads to an improvement in LV contractility $\left(\mathrm{dP} / \mathrm{dt}_{\max }\right)$ and a decrease in $\mathrm{RV} \mathrm{dP} / \mathrm{dt}_{\max }$. An opposite pattern was observed with pre-excitation of the RV. The simulations extended these observations by showing that changes in steady-state BiV cardiac output differed from changes in both LV and RV $\mathrm{dP} / \mathrm{dt}_{\max }$

In this general discussion the results presented in the previous chapters will be placed in a broader perspective. Finally, the impact of this thesis on the clinic as well as on society will be discussed.

\section{POTENTIAL NEW INDICATION FOR PACING THERAPY}

First degree AV-block is commonly considered a benign conduction disorder, however it is known to increase the rate of atrial fibrillation [1], diastolic MR [2] and worse clinical outcome [3] in heart failure patients. The guidelines of the European Society of Cardiology state that restoring AV-coupling using pacemaker therapy should only be considered for patients with persistent symptoms, similar to those of pacemaker syndrome and attributable to a PR-interval >300ms [4]. These guidelines are based on a few small uncontrolled and non-randomized studies, which have suggested that normalization of the AV-delay using conventional DDD pacemakers could reduce diastolic MR and increase cardiac output and LV ejection fraction [5-8]. However, all these studies were performed using permanent $\mathrm{RV}$ pacing, which is known to create pacing-induced heart failure due to dyssynchronous activation of the ventricles. More recently, substudies of the randomized RethinQ [9], MADIT-CRT [10] and COMPANION [11] trials have indicated that non-LBBB patients with prolonged PR-interval do benefit from BiV pacing when compared to unpaced controls. 


\section{AV-coupling in first degree AV-block}

It was against this background that we performed the studies described in chapter 3 . A first interesting and important finding was the similarity in hemodynamic effects of restoring AV-coupling in healthy pigs and heart failure patients. Moreover, these data were quite well replicated by the CircAdapt computer model. The implication of these findings is that they strongly support the physical basis of ventricular filling and that these principles are (largely) the same in normal and failing hearts: passive filling due to AV-pressure gradient, active filling due to atrial contraction and diastolic MR in case there is a reverse AV-pressure gradient. All experimental and simulation data show that LV inflow is reduced when there is fusion of $\mathrm{E}$ - and A-wave, as is the case when the PR-interval is prolonged. Moreover, in all models, PR-prolongation leads to MR, even in healthy hearts. On the other hand, shortening the AV-delay too much, led to a truncation of the A-wave caused by too early ventricular activation leading to a reduction in ventricular filling, indicating that and intermediate, optimal AV-delay represents the best cardiac function.

Moreover, also the translation between improved filling pressures and pump function, through the Frank-Starling mechanism, is comparable between healthy and failing hearts. In the clinical study we showed that restoration of AV-coupling by BiV pacing resulted in a significant increase in stroke work and stroke volume that was most pronounced at an AV-delay of $50 \%$ of the intrinsic PR-interval $(137 \pm 30 \mathrm{~ms})$. We found that the increase in stroke work and cardiac output was obtained by more efficient ventricular filling due to separation of the $\mathrm{E}$ - and A-waves (compared to $\mathrm{E}$-A fusion for long AV-delay), resulting in a greater attribution of the atrial kick. Additionally, the amount of diastolic MR decreased, attributing to better ventricular filling. Optimal ventricular filling leads to improvements in length-dependent activation of the myocardium, which is the cellular basis of the FrankStarling mechanism [12].

The computer simulations and patient studies also showed that the benefits of AV-coupling may be abrogated by pacing-induced ventricular dyssynchrony. Normalizing the AV-delay using RV pacing failed to show improved ventricular filling in patients, possibly explained by prolonged isovolumic contraction and dyssynchronous contraction of the papillary muscles, increasing diastolic MR. In the computer simulations, LV end diastolic volume did increase at optimal AV-delays during RV pacing, but still no improvements in cardiac output or mean arterial pressure were observed, presumably caused by poorer ventricular pump function due to ventricular dyssynchrony. It is therefore important to maintain synchronous activation of the ventricles.

In patients with heart failure a concern may be that restoring AV-coupling leads to an increase in LV end diastolic pressure, possibly giving rise to an increase in pulmonary congestion. However, the data in the porcine hearts and computer simulations show that this increase in LV end diastolic pressure does not coincide with an increase in mean left atrial (LA) pressure, a variable that is probably more related to pulmonary congestion. 
Measurements of in particular LA pressure are cumbersome, the wedge pressure is a surrogate, but does not show the dynamic behavior of this pressure.

Another issue where the porcine experiments enriched the patient study is that stroke volume could be measured using a flow probe mounted around the ascending aorta, thus selectively determining the real forward flow over the aortic valve. In patients, stroke volume calculated from the change in LV volume using the conductance catheter may also include systolic MR, which possibly leads to an overestimation of the stroke volume and thereby underestimate the diastolic MR fraction compared to animals.

\section{Clinical implementation of cardiac pacing for prolonged PR-interval}

Before pacemaker therapy for first degree AV-block can be implemented in the clinic several steps have to be undertaken. First of all, the results presented in chapter $\mathbf{3}$ were all acute hemodynamic improvements. The benefit for cardiac output in this study was comparable to the improvement in cardiac output as seen in conventional CRT, whereas the increase in stroke work was lower $[13,14]$. However, from these studies we also know that acute increases in stroke volume and stroke work in CRT are associated with long term benefits [15]. Secondly, we managed to replicate the abovementioned results in healthy porcine hearts in chapter $\mathbf{3}$ and chapter $\mathbf{4}$. This suggests that optimizing the AV-delay is not only effective for severely symptomatic patients (NYHA class II-III) but also for patients with preserved pump function and mild complaints.

Furthermore, as we hypothesized above, synchronous activation of the ventricles is mandatory and can not only be achieved by BiV pacing, but also by His-bundle pacing [16], left bundle area pacing [17] or LV septal pacing [18]. All these pacing modalities are becoming increasingly popular and make the use of a third LV lead unnecessary and thereby easier and cheaper. Of course, the feasibility, safety and efficacy of pacing-based AV-optimization in patients with first degree AV-block without LBBB and with narrow QRS complex requires additional investigation in prospective trials. These studies should also identify which patient categories benefit most from restoring AV-coupling, and whether the indication should be made based on the ECG derived PR-interval, or the presence of E-A fusion or diastolic MR on echocardiography. Additional studies should also evaluate which pacing modalities are most suitable.

\section{The influence of interatrial delay and interventricular dyssynchrony on the optimal AV-delay}

Chapter 4 further focuses on the mechanisms of AV-delay optimization, with emphasis on atrioventricular timing for both the right and left heart. This evaluation of the whole heart was applied, because total cardiac output depends on the pump function of both ventricles, due to their serial coupling (see also chapter 6). The use of pressure measurements in all four cardiac chambers showed that delays in activation between RA and LA as well as between RV and LV have an impact on optimal pump function of the whole heart. It should 
be noticed that in the majority of studies and in clinical care only attention is paid to the delay between the RA and the RV or LV.

In chapter $\mathbf{4}$ we show that true RA-RV (right effective AV-delay) and LA-LV (left effective AV-delay) delays depend on both IAD and IVD. Because we were aiming at the functional (or mechanical) consequences, we employed the time of maximum rate of rise of each of the chambers to define these effective delays. As expected, RA appendage pacing prolonged IAD (defined as the time difference between RA and LA dP/dt $t_{\text {max }}$ ), leading to a longer optimal AV-delay (defined as the delay providing the highest cardiac output). This finding is in line with previous experiments studying the difference between atrial pacing and atrial sensing [19-22]. Interestingly, in our study the difference in optimal AV-delay between atrial pacing and atrial sensing was the same as the difference in IAD during BiV and RV pacing. In contrast, during LV pacing the difference between the optimal AV-delay between atrial pace and atrial sense mode was larger, which coincided with a greater IVD than during RV and BiV pacing. This might demonstrate that the optimal AV-delay is not only dependent on IAD, but also on IVD. The largest differences in optimal AV-delay were found between RV pacing in atrial sense mode and LV pacing in atrial pace mode. RA pacing leads to delayed activation of the LA, while the LV is pre-excited during LV pacing leading to delayed activation of the RV, creating a long RA-RV delay and a short LA-LV delay. In contrast, during RV pacing in atrial sense mode left and right effective AV-delay are comparable, because the delay of LA activation is corrected by pre-excitation of the RV.

In order to generate an overarching index for optimal AV-delay, accounting for IAD, IVD and the function of both ventricles, we developed the mean effective AV-delay (chapter 4). It turned out that the mean effective AV-delay was better in predicting the optimal AV-delay in pigs than the right or left effective AV-delay alone. The mean effective AVdelay is derived from changes in blood pressures in all four heart chambers and therefore reflects mechanical instead of electrical actions. It represents the time interval between the average time of atrial and average time of ventricular contraction, and by doing so takes into account both the left and the right heart. We believe that the mean effective AV-delay is a way to correct for abnormalities in IAD and IVD in patients and hypothesize that it may be able to improve the CRT effect.

\section{NOVEL OPTIMIZATION STRATEGIES}

Sub-optimal timing of atrial and ventricular activation is one of the causes of ineffective CRT. Optimizing the AV-delay improves ventricular filling and thereby cardiac output and stroke work and there are two ways of doing this. The first is acute optimization, which is performed during or early after the implantation procedure. The golden standard is based on echocardiography, with the optimal AV-delay being considered the shortest AV-delay without truncation of the A-wave. Other optimization strategies use invasively measured pressure-volume loops or first derivative of LV pressure pulse (LV dP/dt), finger 
photoplethysmography, device recorded electrograms or peak endocardial acceleration (SonR) [23]. Unfortunately, no single study was able to show long-term benefits of acute optimization strategies [24-26].

The second way of optimization is continuous, ambulatory optimization with the use of automated algorithms. Currently several automated algorithms are being used that mainly optimize the settings based on electrical parameters. The Adaptive CRT algorithm provides periodic evaluation of intrinsic conduction intervals to optimize LV-only pacing times with intrinsic RV activation [27, 28]. The SyncAV algorithm periodically measures intrinsic conduction delays and programs the AV-delay in such way to create triple wavefront fusion of BiV activation and native conduction [29, 30]. Finally, QuickOpt performs a rapid automatic AV/VV-delay optimization according to intracardiac electrogram data [31]. In chapter $\mathbf{5}$ we studied the SonR system, which is by now the only automated algorithm based on mechanical data being used for CRT. The CLEAR and RESPOND-CRT randomized trials have shown that optimization of CRT using the SonR system is non-inferior in terms of responder rate and hospitalization in heart failure patients with wide QRS complex compared to standard echocardiography-based optimization [32-34]. One of the main aims of this thesis was to find improvements for AV-optimization.

\section{Using the mean effective AV-delay to optimize pacemaker settings}

As discussed above and in chapter $\mathbf{4}$, the mean effective AV-delay takes into account the mechanical contractions of both atria and both ventricles, which makes it a more reliable parameter to define the pacemaker setting with the highest overall cardiac output. In theory, this parameter can be used for acute optimization during the implantation procedure, although it would need invasive blood pressure monitoring in all heart chambers.

Alternatively, doppler echocardiography might be a non-invasive tool to define the RA-RV and LA-LV intervals. The continuous wave doppler signal over the mitral and tricuspid valve gives information on the moment of $L A$ and $R A$ activation based on the A-wave. The timing of LV and RV contraction and subsequently IVD can be derived from the moments of aortic and pulmonary valve opening, which resemblances the time between LV and

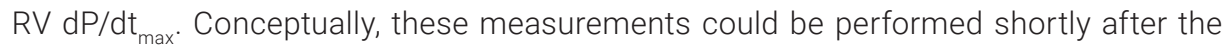
pacemaker implantation while BiV pacing at various AV-delays during atrial sense or atrial pace mode. In the porcine model the mean effective AV-delay was 27\% of the RR-interval (HR 100bmp), while in Circadapt it was 14\% (HR 60-100bpm). For patients, however, this value still needs to be determined before it can be used for optimization strategies.

The above-described parameters are all echo-derived mechanical parameter that can unfortunately not be used for ambulatory, continuous optimization using automated algorithms. It would be interesting to examine whether electrical parameters can be used as surrogates for mechanical actions. For instance, the RA-RV interval can be derived from the RA and RV lead, while the P-wave width gives information about the IAD. Additionally, 
the left sided AV-delay can be derived from the IAD and sensing of the LV wall, although this requires an LV lead and only gives information about activation of the posterolateral wall. Additional prospective studies are required to prove the benefits of these electrical markers and whether they support the importance of the mean effective AV-delay.

\section{Unraveling the SonR signal}

Mechanical signals of the heart can also be captured by intracardiac accelerometers and are currently used for ambulatory optimization of CRT. The SonR system uses the SonR1 signal, which corresponds to the first heart sound. Heart sounds are acoustic vibrations generated by mechanical interactions of the cardiohemic structures, like valves, myocardium and blood mass. In the 1960s Rushmer suggested that abrupt cessation of backward flow by each valve closure caused vibrations of the blood and surrounding tissue, including valve leaflets and atrial, ventricular and arterial walls [35]. In case of the first heart sound, the vibrations are then caused by closure of the mitral and tricuspid valves.

The SonR1 amplitude increases during improved ventricular contraction, as shown in chapter $\mathbf{5}$ in a canine heart failure model, and previously in pigs and sheep [36-38]. In CRT, however, SonR1 behaves differently because changes in AV- and VV-delay create variations in diastolic filling and IVD. Like previously reported, the SonR1 amplitude increases at short AV-delays when atrial contraction occurs against closed or quickly closing atrioventricular valves $[39,40]$. These data reveal that SonR1 amplitude does not only depend on the amount of contractility but also the interval between atrial and ventricular contraction, supporting Rushmer's theory.

The SonR algorithm is complex and defines the optimal AV-delay as the shortest AVdelay with the lowest SonR1 amplitude; the so-called infliction point. The optimal AVdelay as defined using SonR strongly correlates to the optimal AV-delay as derived using echocardiographic measurement, like longest diastolic filling time and aortic velocity time integral [41-43]. In the clinical setting the algorithm is non-inferior in terms of responder rate compared to echocardiography-based AV-optimization and decreases heart failure hospitalization by $35 \%[32,34]$. Our goal was to better understand the SonR algorithm.

Chapter $\mathbf{5}$ shows that we replicated the sigmoid curve of the SonR1 amplitude at various AV-delays in a canine heart failure model. The AV-delay at which the infliction point of the AV-delay - SonR1 curve occurs was hard to determine due to measurement variability and due to the fact that this point is at the shallow part of the curve. However, the highest values of maximal LV pressure and LV dP/dt ${ }_{\max }$ occurred at AV-delays just longer that the one where SonR1 significantly increased (comparable to the infliction point), supporting the use of the SonR1 algorithm for AV-optimization. Additionally, chapter $\mathbf{5}$ reveals that the SonR1 signal does not seems to be influenced by IVD, as the amplitudes during BiV pacing are comparable as those during LV pacing with greater amount of IVD, suggesting that it should not be used for V V-optimization. 
The amplitude of the SonR signal as used in chapter $\mathbf{5}$ is larger in the RV than RA location, comparable to previous studies [44-48]. We hypothesize that the position of the sensor defines which part of the heart dominates the signal. When the SonR sensor is placed in the RA lead it is able to give information on both ventricles because of its remote position, while a sensor placed in the RV might focus more on RV function than LV function. Because of this hypothesis it is expected that positioning the sensor in the RA lead will be more reliable in defining the optimal AV-delay because it gives information of both ventricles. Since the SonR1 signal is compromised out of a tricuspid and mitral valve component it could well be that the signal becomes wider during a greater amount of IVD. Future research should focus on how the different components of the SonR signal can be used to obtain better timing of atrial and ventricular contraction. Also, the second heart sound (or SonR2), which is a consequence of aortic and pulmonary valve closure might aid in better synchronization of the heart by analyzing splitting of the aortic and pulmonary component [49].

The SonR system is currently clinically used for AV-and VV-delay optimization in CRT patients. Of course, when first degree AV-block (chapter 3) will be seen as a new indication for pacing therapy, additional research has to be performed to see whether this algorithm is successful in this new patient category. Much will depend on ongoing research involving the location of the ventricular leads. In case of His bundle, LV septal or LBB area pacing only one ventricular pacemaker lead will be present, meaning optimization algorithms can focus on optimizing AV-delay.

\section{THE IMPORTANCE OF THE RIGHT VENTRICLE}

Optimization of CRT or other pacing therapies mainly focusses on the LV, while the RV is often overlooked. In chapter 6 we studied the changes in LV and RV dP/dt $t_{\text {max }}$ during variations of $\mathrm{AV}$ - and $\mathrm{VV}$-delays in a canine total AV-block model. LV dP/dt max $_{\text {ax }}$ showed the largest increase compared to the reference setting during LV pre-excitation, while RV dP/ $\mathrm{dt}_{\max }$ was highest with RV pre-excitation. Interestingly, the setting with the highest cardiac output differed from the settings with the best LV or RV dP/dt $\mathrm{dmax}_{\text {in }}$ computer simulations, indicating that improving LV contractility through LV pre-excitation does not necessarily lead to the best overall cardiac pump function. Since the RV and LV are coupled in series, decreasing RV function due to LV pre-excitation leads to reduced pre-load of the LV and thereby a decrease in cardiac output. We found that the settings with the highest cardiac output were during simultaneous pacing of both ventricles, suggesting that overall cardiac function is a compromise of left and right function.

In chapter 4 we proposed the mean effective AV-delay as better predictor for AVoptimization. This parameter is a combination of left and right AV-delay, indicating once again that also RA-RV coupling is important to obtain optimal whole heart cardiac function. Also chapter $\mathbf{5}$ gives clues that the RV is important as the SonR lead placed in the RA that 'oversees' both ventricles is better that the RV SonR1 signal in predicting the optimal AV- 
delay. With this knowledge, the improvements in stroke during BiV pacing in patients with first degree AV-block as presented in chapter $\mathbf{3}$ might be even bigger when an optimal VV-delay instead of LV pre-excitation (-40ms) was used. In future, the RV function should therefore be part of AV-optimization strategies.

\section{ANIMAL MODELS FOR PACING RESEARCH}

Animals have been used for research purposes since the development of the pacemaker in the 1950s. Nowadays animals are used to study ventricular dyssynchrony and resynchronization therapy. Main advantage of animal use over patients is that invasive experiments can be more complex and extensive.

In chapter $\mathbf{4}$ for example we were able to measure pressures in all four heart chambers and the aorta and additionally we could also measure flow in the ascending aorta. In chapter $\mathbf{5}$ the SonR signal could be derived from various locations of the heart while invasive pressure and flow measurements were performed. These invasive measurements are not possible in patients, and thereby give us extra information that cannot be derived from clinical experiments.

In this thesis two animal species have been used: dogs and pigs. Historically, dogs have been the species of choice for this kind of research, in particular because ventricular pacing induced a degree of dyssynchrony comparable to humans [50]. On Pubmed the search terms [ventricular dyssynchrony dog/canine] results in 102 hits, where replacing $\mathrm{dog} /$ canine by pig/porcine results in 38 hits. However, during recent years the use of dogs for research was banned or avoided due to increasing societal protests. At Maastricht University, dog experiments were stopped just at the onset of this PhD project. This thesis therefore contains data from both species and allows to compare the electrophysiological and hemodynamic consequences of dyssynchrony in both species.

In previous canine studies the QRS almost doubled to 113ms when creating a LBBB [51], but unfortunately these values, obtained at a French research center, were not achieved in chapter 5, possibly because of smaller size and/or different race of the dogs. In pigs (chapter 4) increase in QRS duration due to ventricular pacing was smaller than those in dogs [52]. Maximal IVD during LV pacing in this porcine model was 18ms, while the IVD in the LBBB or RV-lateral wall paced (mimicking LBBB) canines (chapter 5) were remarkably larger ( 33ms). Additionally, it is known that LV dP/dt max improvement as achieved by CRT is significantly larger in canines compared to pigs [53].

These data show that pigs have considerable limitations regarding their use for studies on dyssynchrony, but knowing these limitations, the species can still be of use, for example investigating the effect of prolonged PR-interval (chapter $\mathbf{3}$ and chapter $\mathbf{4}$ ). It is unlikely that dogs can be used again for cardiac research. The present thesis does show that there may be an alternative: computer models like CircAdapt. In chapter $\mathbf{6}$ we used existing dog 
data for developing a dog-specific computer model. Now this has been developed, studies may be performed in this model instead of animals.

\section{CONCLUSION}

Based on this thesis we can conclude that restoration of AV-coupling using BiV pacing in heart failure patients and pigs with first degree AV-block leads to significant improvements of LV hemodynamics, based on optimal ventricular filling due to an increase in forward flow and a decrease in diastolic mitral regurgitation. This may lead to a novel pacing-based therapeutic option in patients with heart failure and prolonged PR-interval. Furthermore, this suggests that AV-delay optimization remains an important factor in improving efficacy of pacemaker therapies like CRT. While optimizing the AV-delay one should not only focus on the left side of the heart, but also pay attention to the right side. Additionally, as the optimal AV-delay is influenced by interatrial delay and interventricular dyssynchrony, the mean effective AV-delay could be a new parameter for AV-delay optimization. Ultimately, ambulatory optimization of programmed AV-delays should focus on left and right sided AV-delays and make use of a combination of electrical and mechanical parameters. 


\section{REFERENCES}

1 Cheng S, Keyes MJ, Larson MG, McCabe EL, Newton-Cheh C, Levy D, et al. Long-term outcomes in individuals with prolonged PR interval or first-degree atrioventricular block. JAMA. 2009;301(24):2571-7.

2 Ishikawa T, Kimura K, Miyazaki N, Tochikubo O, Usui T, Kashiwagi M, et al. Diastolic mitral regurgitation in patients with first-degree atrioventricular block. Pacing Clin Electrophysiol. 1992;15(11 Pt 2):1927-31.

3 Park SJ, On YK, Byeon K, Kim JS, Choi JO, Choi DJ, et al. Short- and long-term outcomes depending on electrical dyssynchrony markers in patients presenting with acute heart failure: clinical implication of the first-degree atrioventricular block and QRS prolongation from the Korean Heart Failure registry. Am Heart J. 2013;165(1):57-64 e2.

4 Brignole M, Auricchio A, Baron-Esquivias G, Bordachar P, Boriani G, Breithardt OA, et al. 2013 ESC guidelines on cardiac pacing and cardiac resynchronization therapy: the task force on cardiac pacing and resynchronization therapy of the European Society of Cardiology (ESC). Developed in collaboration with the European Heart Rhythm Association (EHRA). Europace. 2013;15(8):1070-118.

5 Brecker SJ, Xiao HB, Sparrow J, Gibson DG. Effects of dual-chamber pacing with short atrioventricular delay in dilated cardiomyopathy. Lancet. 1992;340(8831):1308-12.

6 Hochleitner M, Hortnagl H, Hortnagl H, Fridrich L, Gschnitzer F. Long-term efficacy of physiologic dual-chamber pacing in the treatment of end-stage idiopathic dilated cardiomyopathy. Am J Cardiol. 1992;70(15):1320-5.

7 Ishikawa T, Sumita S, Kimura K, Kikuchi M, Kosuge M, Nakagawa T, et al. Efficacy of atrioventricular sequential pacing and diastolic mitral regurgitation in patients with intrinsic atrioventricular conduction. Jpn Circ J. 2000;64(8):579-82.

8 Sack S, Franz R, Dagres N, Oldenburg O, Herrmann J, Golles A, et al. Can right-sided atrioventricular sequential pacing provide benefit for selected patients with severe congestive heart failure? Am J Cardiol. 1999;83(5B):124D-9D.

9 Joshi NP, Stopper MM, Li J, Beshai JF, Pavri BB. Impact of baseline PR interval on cardiac resynchronization therapy outcomes in patients with narrow QRS complexes: an analysis of the ReThinQ Trial. J Interv Card Electrophysiol. 2015;43(2):145-9.

10 Lin J, Buhr KA, Kipp R. Effect of PR Interval on Outcomes Following Cardiac Resynchronization Therapy: A Secondary Analysis of the COMPANION Trial. J Cardiovasc Electrophysiol. 2017;28(2):185-91.

11 Stockburger M, Moss AJ, Klein HU, Zareba W, Goldenberg I, Biton Y, et al. Sustained clinical benefit of cardiac resynchronization therapy in non-LBBB patients with prolonged PR-interval: MADIT-CRT long-term follow-up. Clin Res Cardiol. 2016;105(11):944-52.

12 Sequeira V, van der Velden J. Historical perspective on heart function: the Frank-Starling Law. Biophys Rev. 2015;7(4):421-47.

13 de Roest GJ, Allaart CP, Kleijn SA, Delnoy PP, Wu L, Hendriks ML, et al. Prediction of long-term outcome of cardiac resynchronization therapy by acute pressure-volume loop measurements. Eur J Heart Fail. 2013;15(3):299-307.

14 Mafi Rad M, Blaauw Y, Prinzen FW, Vernooy K. The role of acute invasive haemodynamic measurements in cardiac resynchronization therapy: looping towards prediction of long-term response and therapy optimization. Eur J Heart Fail. 2013;15(3):247-9.

15 Zweerink A, Salden OAE, van Everdingen WM, de Roest GJ, van de Ven PM, Cramer MJ, et al. Hemodynamic Optimization in Cardiac Resynchronization Therapy: Should We Aim for dP/ dtmax or Stroke Work? JACC Clin Electrophysiol. 2019;5(9):1013-25.

16 Arnold A, Shun-Shin M, Keene D, Howard J, Sohaib SMA, Wright I, et al. His Resynchronization Versus Biventricular Pacing in Patients With Heart Failure and Left Bundle Branch Block. J Am Coll Cardiol. 2018;72(24):3112-22. 
17 Vijayaraman P, Subzposh FA, Naperkowski A, Panikkath R, John K, Mascarenhas V, et al. Prospective evaluation of feasibility, electrophysiologic and echocardiographic characteristics of left bundle branch area pacing. Heart Rhythm. 2019.

18 Salden F, Luermans J, Westra SW, Weijs B, Engels EB, Heckman LIB, et al. Short-Term Hemodynamic and Electrophysiological Effects of Cardiac Resynchronization by Left Ventricular Septal Pacing. J Am Coll Cardiol. 2020;75(4):347-59.

19 Dabrowska-Kugacka A, Lewicka-Nowak E, Rucinski P, Zagozdzon P, Raczak G, Kutarski A. Relationship between P-wave duration and atrial electromechanical delay assessed by tissue Doppler echocardiography. Pacing Clin Electrophysiol. 2011;34(1):23-31.

20 Janosik DL, Pearson AC, Buckingham TA, Labovitz AJ, Redd RM, Mrosek D. The hemodynamic benefit of differential atrioventricular delay intervals for sensed and paced atrial events during physiologic pacing. J Am Coll Cardiol. 1989;14(2):499-507.

21 Kyriacou A, Rajkumar CA, Pabari PA, Sohaib SMA, Willson K, Peters NS, et al. Distinct impacts of heart rate and right atrial-pacing on left atrial mechanical activation and optimal AV delay in CRT. Pacing Clin Electrophysiol. 2018.

22 Wish M, Fletcher RD, Gottdiener JS, Cohen AI. Importance of left atrial timing in the programming of dual-chamber pacemakers. Am J Cardiol. 1987;60(7):566-71.

23 Houthuizen P, Bracke FA, van Gelder BM. Atrioventricular and interventricular delay optimization in cardiac resynchronization therapy: physiological principles and overview of available methods. Heart Fail Rev. 2011;16(3):263-76.

24 Vernooy K, van Deursen CJ, Strik M, Prinzen FW. Strategies to improve cardiac resynchronization therapy. Nat Rev Cardiol. 2014;11(8):481-93.

25 Zhang Y, Xing Q, Zhang JH, Jiang WF, Qin M, Liu X. Long-Term Effect of Different Optimizing Methods for Cardiac Resynchronization Therapy in Patients with Heart Failure: A Randomized and Controlled Pilot Study. Cardiology. 2019;142(3):158-66.

26 Abraham WT, Gras D, Yu CM, Calo L, Islam N, N K. Results from the FREEDOM trial: assess the safety and efficacy of frequent optimization of cardiac resynchronization therapy. Presented at Heart Rhythm Society 31st Annual Scientific Sessions Denver. 2010.

27 Krum H, Lemke B, Birnie D, Lee KL, Aonuma K, Starling RC, et al. A novel algorithm for individualized cardiac resynchronization therapy: rationale and design of the adaptive cardiac resynchronization therapy trial. Am Heart J. 2012;163(5):747-52 e1.

28 Martin DO, Lemke B, Birnie D, Krum H, Lee KL, Aonuma K, et al. Investigation of a novel algorithm for synchronized left-ventricular pacing and ambulatory optimization of cardiac resynchronization therapy: results of the adaptive CRT trial. Heart Rhythm. 2012;9(11):1807-14.

29 Thibault B, Ritter P, Bode K, Calo L, Mondesert B, Mangual JO, et al. Dynamic programming of atrioventricular delay improves electrical synchrony in a multicenter cardiac resynchronization therapy study. Heart Rhythm. 2019;16(7):1047-56.

30 Varma N, O’Donnell D, Bassiouny M, Ritter P, Pappone C, Mangual J, et al. Programming Cardiac Resynchronization Therapy for Electrical Synchrony: Reaching Beyond Left Bundle Branch Block and Left Ventricular Activation Delay. J Am Heart Assoc. 2018;7(3).

31 Wang J, Liang Y, Chen H, Wang W, Bai J, Chen X, et al. Patient-tailored SyncAV algorithm: A novel strategy to improve synchrony and acute hemodynamic response in heart failure patients treated by cardiac resynchronization therapy. J Cardiovasc Electrophysiol. 2020;31(2):512-20.

32 Ritter P, Delnoy PP, Padeletti L, Lunati M, Naegele H, Borri-Brunetto A, et al. A randomized pilot study of optimization of cardiac resynchronization therapy in sinus rhythm patients using a peak endocardial acceleration sensor vs. standard methods. Europace. 2012;14(9):1324-33.

33 Brugada J, Brachmann J, Delnoy PP, Padeletti L, Reynolds D, Ritter P, et al. Automatic optimization of cardiac resynchronization therapy using SonR-rationale and design of the clinical trial of the SonRtip lead and automatic AV-VV optimization algorithm in the paradym RF SonR CRT-D (RESPOND CRT) trial. Am Heart J. 2014;167(4):429-36.

34 Brugada J, Delnoy PP, Brachmann J, Reynolds D, Padeletti L, Noelker G, et al. Contractility sensor-guided optimization of cardiac resynchronization therapy: results from the RESPONDCRT trial. Eur Heart J. 2016. 
35 Rushmer RF, Morgan C. Meaning of murmurs. Am J Cardiol. 1968;21(5):722-30.

36 Rickards AF, Bombardini T, Corbucci G, Plicchi G. An implantable intracardiac accelerometer for monitoring myocardial contractility. The Multicenter PEA Study Group. Pacing Clin Electrophysiol. 1996;19(12 Pt 1):2066-71.

37 Bordachar P, Garrigue S, Reuter S, Hocini M, Kobeissi A, Gaggini G, et al. Hemodynamic assessment of right, left, and biventricular pacing by peak endocardial acceleration and echocardiography in patients with end-stage heart failure. Pacing Clin Electrophysiol. 2000;23(11 Pt 2):1726-30.

38 Plicchi G. PEA I and PEA II based implantable haemodynamic monitor: pre clinical studies in sheep. Europace. 2002;4(1):49-54.

39 Shah PM, Kramer DH, Gramiak R. Influence of the timing of atrial systole on mitral valve closure and on the first heart sound in man. Am J Cardiol. 1970;26(3):231-7.

40 Stept ME, Heid CE, Shaver JA, Leon DF, Leonard JJ. Effect of altering P-R interval on the amplitude of the first heart sound in the anesthetized dog. Circ Res. 1969;25(3):255-63.

41 Ritter P, Padeletti L, Gillio-Meina L, Gaggini G. Determination of the optimal atrioventricular delay in DDD pacing. Comparison between echo and peak endocardial acceleration measurements. Europace. 1999;1(2):126-30.

42 Dupuis JM, Kobeissi A, Vitali L, Gaggini G, Merheb M, Rouleau F, et al. Programming optimal atrioventricular delay in dual chamber pacing using peak endocardial acceleration: comparison with a standard echocardiographic procedure. Pacing Clin Electrophysiol. 2003;26(1 Pt 2):210-3.

43 Padeletti L, Porciani MC, Ritter P, Michelucci A, Colella A, Pieragnoli P, et al. Atrioventricular interval optimization in the right atrial appendage and interatrial septum pacing: a comparison between echo and peak endocardial acceleration measurements. Pacing Clin Electrophysiol. 2000;23(11 Pt 1):1618-22.

44 Bordachar P, Garrigue S, Ritter P, Ploux S, Labrousse L, Casset C, et al. Contributions of a hemodynamic sensor embedded in an atrial lead in a porcine model. J Cardiovasc Electrophysiol. 2011;22(5):579-83.

45 Bombardini T, Gaggini G, Marcelli E, Parlapiano M, Plicchi G. Peak endocardial acceleration reflects heart contractility also in atrial fibrillation. Pacing Clin Electrophysiol. 2000;23(9):1381-5.

46 Duncker D, Delnoy PP, Nagele H, Mansourati J, Mont L, Anselme F, et al. First clinical evaluation of an atrial haemodynamic sensor lead for automatic optimization of cardiac resynchronization therapy. Europace. 2015.

47 Gras D, Kubler L, Ritter P, Anselme F, Delnoy PP, Bordachar P, et al. Recording of peak endocardial acceleration in the atrium. Pacing Clin Electrophysiol. 2009;32 Suppl 1:S240-6.

48 Bongiorni MG, Soldati E, Arena G, Quirino G, Vernazza F, Bernasconi A, et al. Is local myocardial contractility related to endocardial acceleration signals detected by a transvenous pacing lead? Pacing Clin Electrophysiol. 1996;19(11 Pt 2):1682-8.

49 Luo H, Westphal P, Shahmohammadi M, Heckman LIB, Kuiper M, Cornelussen RN, et al. Second heart sound splitting as an indicator of interventricular mechanical dyssynchrony using a novel splitting detection algorithm. Physiol Rep. 2021;9(1):e14687.

50 Strik M, van Middendorp LB, Vernooy K. Animal models of dyssynchrony. J Cardiovasc Transl Res. 2012;5(2):135-45.

51 Verbeek XA, Vernooy K, Peschar M, Cornelussen RN, Prinzen FW. Intra-ventricular resynchronization for optimal left ventricular function during pacing in experimental left bundle branch block. J Am Coll Cardiol. 2003;42(3):558-67.

52 Strik M, van Deursen CJ, van Middendorp LB, van Hunnik A, Kuiper M, Auricchio A, et al. Transseptal conduction as an important determinant for cardiac resynchronization therapy, as revealed by extensive electrical mapping in the dyssynchronous canine heart. Circ Arrhythm Electrophysiol. 2013;6(4):682-9.

53 Heckman LIB, Kuiper M, Anselme F, Ziglio F, Shan N, Jung M, et al. Evaluating multisite pacing strategies in cardiac resynchronization therapy in the preclinical setting. Heart Rhythm 02. 2020;1(2):111-9. 


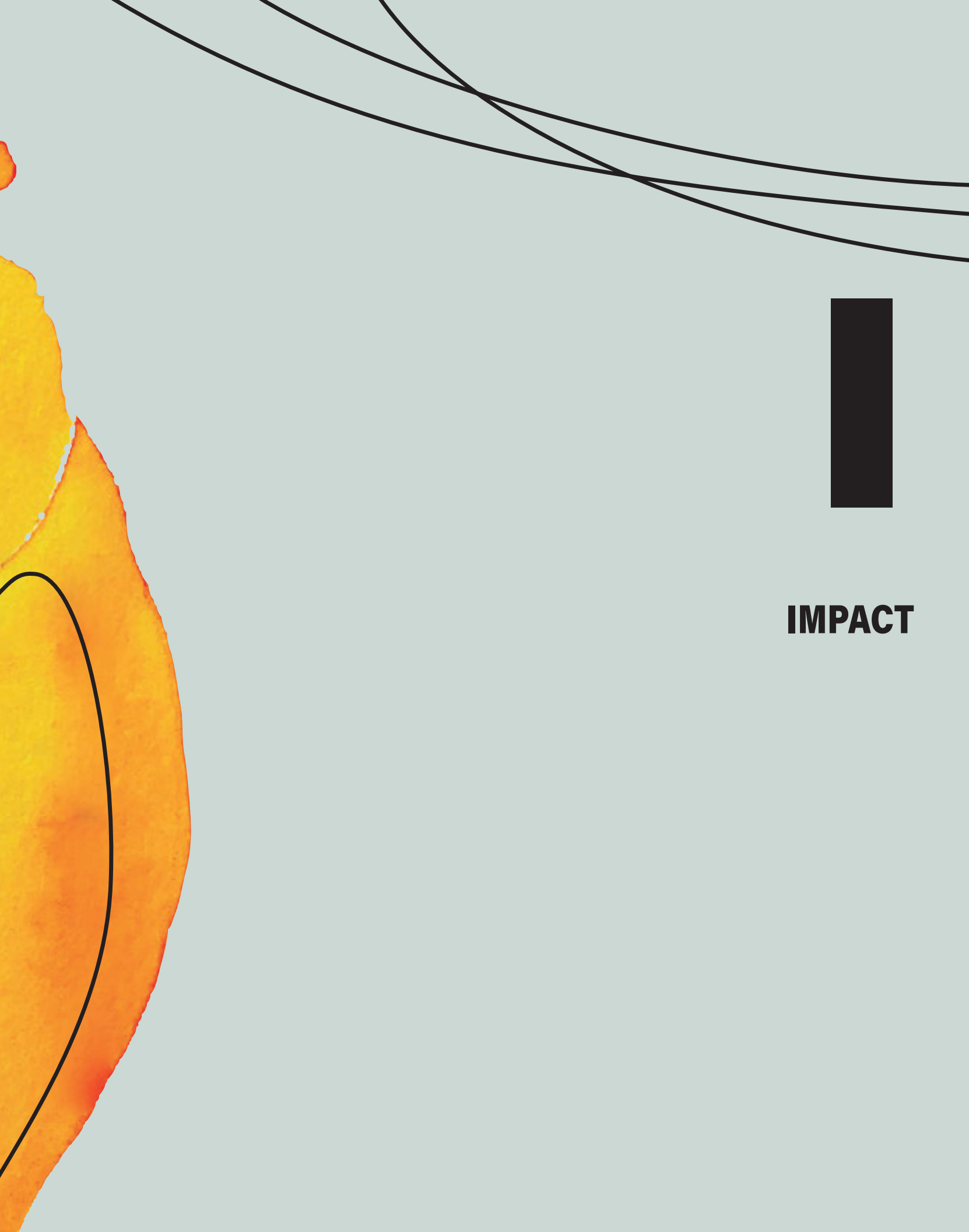

IMPACT 



\section{IMPACT}

In 2019 nearly 250.000 people suffered from heart failure in the Netherlands, leading to almost 30.000 cases of hospitalization [1]. All-cause-mortality in patients with heart failure is $7 \%$ and even increases to $17 \%$ in case of hospitalization [2]. The total health care costs of heart failure in the Netherlands were 817 million euros in 2017 [1]. Besides trying to take away the underlying cause of heart failure, treatment consists of lifestyle changes and pharmacotherapy to reduce cardiac stress and filling pressures. Approximately a quarter of all patients with heart failure also have electrical conduction disorders, mainly left bundle branch block (LBBB). These patients may benefit from cardiac resynchronization therapy (CRT) as adjunct therapy [3]. CRT synchronizes the electrical activation and thereby contraction of the left and right ventricle (RV) using biventricular (BiV) pacing and thereby improve the overall cardiac output. In the Netherlands about 12.500 pacemakers were implanted in 2019 of which 2.500 were CRT devices [4]. Although CRT provides great reductions in heart failure burden on the population level, individual response varies significantly. The main objectives of this thesis were: 1) to study whether BiV pacing could also improve cardiac function in patients with first-degree AV-block and 2) to examine how the optimal benefit of CRT could be achieved by optimizing the delay between atrial and ventricular activation (AV-delay). In this chapter we summarize the clinical and societal impact of the main findings of this thesis.

\section{Clinical impact}

In chapter $\mathbf{3}$ we showed that restoring physiological AV-delays using BiV pacing increased cardiac pump function in heart failure patients with first-degree AV-block who did not have clear intraventricular conduction disorders. Therefore, this acute hemodynamic study suggests that indications for CRT may be extended to patients who have heart failure and a first-degree AV-block. Obviously, larger and long-term studies are required to support this idea before it can be added to the CRT-guidelines. Additionally, the results in chapter $\mathbf{3}$ implicated that the benefits that were achieved by restoring AV-coupling were reduced by cardiac dyssynchrony as induced by RV pacing, indicating that restoration of AV coupling by pacing therapy should be performed while maintaining as much as possible the normal ventricular conduction.

In chapter $\mathbf{3}$ we also showed that optimizing the AV-delay in a porcine healthy heart resulted in greater cardiac output. This implicates that patients with first-degree AV-block but without heart failure might benefit from cardiac pacing as well. We hypothesize that pacing therapy in these patients does not necessarily treat symptoms, but may prevent the development of heart failure, and thereby decreases morbidity and mortality. Again, before this therapy can be broadly introduced in the clinic, additional research and preferably randomized controlled trials need to be done. These studies should not only focus on which patients are most eligible for the therapy but also which pacing modality shows the optimal result. As mentioned before maintaining ventricular synchrony is a must, something which 
can, besides BiV pacing, also be achieved by His-bundle, left bundle branch area, or LV septum pacing as well.

\section{Scientific impact}

In chapter $\mathbf{3}$ and chapter $\mathbf{4}$ the hemodynamic changes that occurred due to AV-delay optimization were thoroughly studied. As known from basic physiology, better filling of the ventricles leads to pre-stretching of the cardiac myocytes which in response leads to improved contraction, conform the Frank-Starling mechanism [5]. Our studies in chapter 3 showed that in case of first-degree AV-block LV filling was improved due to a combination of increased forward flow over the mitral valve and a reduction in the amount of diastolic mitral regurgitation (MR). Therefore, also in future studies both these processes require attention.

Chapter $\mathbf{4}$ expanded these findings, by investigating the AV-coupling for both the right and left heart. In this regard it should be noticed that conventionally the AV-delay is defined by the time between stimulating (or sensing activity) the RA and stimulation of one or two of the ventricles. We showed that there are considerable differences in "effective" AV-delay between the right and left side of the heart (RA-RV vs. LA-LV delays) and that these delays also strongly depend on the site of ventricular pacing. The mean effective AV-delay takes into account the right and left side of the heart, and was able to more accurately predict the optimal AV-delay. Implication for future research is that both atrial (interatrial delay) and ventricular (interventricular dyssynchrony) activation sequences should be taken into account when searching for new algorithms for optimization of AV-and VV-delays.

In chapter $\mathbf{5}$ we studied the SonR signal which is derived from intracardiac accelerations and gives mechanical information of cardiac contractility. We proved that the amplitude of this signal is not only sensitive for ventricular contraction, but also changes with alterations in ventricular filling at various AV-delays. On the contrary, we proved that the influence of interventricular dyssynchrony on the amplitude of the signal is small. Chapter $\mathbf{6}$ shows that LV pre-excitation improves LV function but impairs RV function, while RV pre-excitation causes an improvement in $\mathrm{R} V$ function and a reduction of $\mathrm{LV}$ function. This indicates again that both ventricles should be taken into account while optimizing the AV-delay.

\section{Societal impact}

Part of the potential societal impact of this work relates to the ideas about improving pacemaker therapies. Doing so we believe that health care costs may be reduced due to less symptoms and decreased hospitalization rate. However, as mentioned above, there are several steps to be taken before this benefit can be proven.

This thesis also demonstrates how clinical and animal experiments can be enhanced through computer simulations. In chapter $\mathbf{3}$ computer simulations aided in better understanding the hemodynamic improvements caused by AV-coupling restoration. In 
chapter $\mathbf{6}$ animal data were the base for computer simulations showing that RV function is important for overall cardiac function. And the results from the animal study in chapter 4 have already been replicated using the CircAdapt software, as well. This shows that computer models have a future in pacemaker research. They can be used in hypothesis generation or help in simulating experiments that are not possible in humans or animals. Multidisciplinary collaboration between cardiologists, physiologists and biomedical engineers, is key to gain the optimal result from computer modeling and simulation.

Currently, data from animal and experimental work is still necessary for the development, validation and improvement of models, like the CircAdapt model. On the other hand, these computer models can help to reduce the number of animal and clinical studies by improving hypotheses and gaining more accurate inside in hemodynamics. It is to be expected that in this field the number of animal experiments can decrease over time, while the contribution of computer models will increase. 


\section{REFERENCES}

1 Volksgezondheid en zorg - Hartfalen. 2021 [Available from: https://www.volksgezondheidenzorg. info/onderwerp/hartfalen.

2 Maggioni AP, Dahlstrom U, Filippatos G, Chioncel O, Crespo Leiro M, Drozdz J, et al. EURObservational Research Programme: regional differences and 1-year follow-up results of the Heart Failure Pilot Survey (ESC-HF Pilot). Eur J Heart Fail. 2013;15(7):808-17.

3 Brignole M, Auricchio A, Baron-Esquivias G, Bordachar P, Boriani G, Breithardt OA, et al. 2013 ESC guidelines on cardiac pacing and cardiac resynchronization therapy: the task force on cardiac pacing and resynchronization therapy of the European Society of Cardiology (ESC). Developed in collaboration with the European Heart Rhythm Association (EHRA). Europace. 2013;15(8):1070-118.

4 NHR Rapportage 2020. 2020 [Available from: https://nederlandsehartregistratie.nl/wp-content/ uploads/2020/11/NHR-Rapportage-2020-231120.pdf.

5 Sequeira V, van der Velden J. Historical perspective on heart function: the Frank-Starling Law. Biophys Rev. 2015;7(4):421-47. 





\section{SUMMARY}

Cardiac conductions disorders like left bundle branch block (LBBB) and first-degree atrioventricular (AV)-block may lead to diminished cardiac function and can ultimately lead to heart failure. Patients with LBBB with increased QRS duration (>130ms) and reduced left ventricular (LV) ejection fraction (<35\%) are candidates for cardiac resynchronization therapy (CRT). The goal of CRT is to synchronize the electrical activation of both ventricles, ultimately leading to improved cardiac function. Patients benefit exists of decreased mortality and hospitalization rate and an increase in quality of life. However, at the individual level approximately a quarter of patients fulfilling the abovementioned criteria do not respond to this therapy. The response to CRT depends on several factors, one of them being the pacemaker setting for timing of atrial and ventricular activation. Optimization of these settings is often performed only once after the implantation of the pacemaker, but algorithms that enable ambulatory optimization have been introduced in recent years. Most of them are based on ECG or device-derived electrograms (like SyncAV, Adaptive CRT and QuickOpt), while there is one algorithm available that employs mechanical information derived from an accelerometer in a pacemaker lead (SonR). The application of CRT for patients with a wide QRS complex is well established, while considerably less is known about its use in patients with prolonged PR-interval (first-degree AV-block) and narrow QRS complex. The promising data here are until now only derived from subanalyses of randomized CRT-trials.

The thesis as presented here has two main objectives. The first aim is to examine whether CRT also improves cardiac function in patients and animals with prolonged PR-interval and whether first-degree AV-block could be a new indication for this form of pacing therapy. The second objective is to explore new ways of optimizing pacemaker setting in CRT and relate them to acute hemodynamic outcome.

In chapter 2 we start by reviewing the electrophysiological and hemodynamic changes that occur during CRT in both animals and patients. LBBB leads to interventricular dyssynchrony (IVD) with prolonged total activation time of the ventricles and causes wasted work. Next, we explain how CRT reduces this electrical dyssynchrony and results in an acute

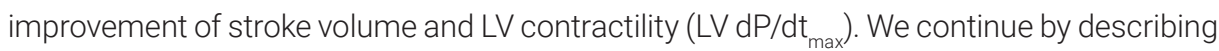
how pacing locations and alternative pacing modalities like multipoint and endocardial pacing influence the effect of CRT. Finally, the importance of optimizing pacemaker settings is explored by showing hemodynamic changes during a great variety of $\mathrm{AV}$ - and $\mathrm{VV}$-delays.

The goal of chapter $\mathbf{3}$ is to examine the effect of restoring AV-coupling in hearts with firstdegree AV-block using animal experiments, computer modeling and patient studies. In the animal experiment we show that optimizing the AV-delay using biventricular (BiV) pacing, results in a significant increase in mean arterial pressure and cardiac output, compared to the reference setting with a prolonged AV-delay of $300 \mathrm{~ms}$. Similar results are found in heart 
failure patients with first-degree AV-block (PR-interval >230ms) and QRS duration < 150ms. Using invasive pressure-volume loops, we find that the improvements in hemodynamics are achieved by better LV filling, which is the result of a combination of increased forward flow over the mitral valve and a reduction in diastolic mitral regurgitation. These results are replicated using the CircAdapt computer model.

In the patient study we show that restoring AV coupling using BiV pacing increases stroke volume and stroke work, but that these improvements are absent when using right ventricular (RV) pacing. This adverse effect of RV pacing, caused by increased ventricular dyssynchrony, is also seen in CircAdapt simulations. These results indicate that restoration of AV-coupling may provide considerable benefit to patients, as long as an increase in ventricular dyssynchrony is avoided.

The aim of chapter $\mathbf{4}$ is to get a better understanding of the diastolic filling patterns during various AV-delays. Furthermore, it explores how variations in interatrial delay (IAD) and various ventricular pacing sites with changes in IVD influence the optimal AV-delay. The ultimate goal is to better predict the optimal AV-delay using parameters based on effective AV-delays of the right and left heart. Experiments were performed in a porcine total AVblock model in which atrial pacing was compared to atrial sensing for RV, LV and BiV pacing at various AV-delays. Our findings are that the optimal AV-delay is a function of both atrial sensing or atrial pacing and of the ventricular pacing site, so a function of both IAD and IVD. To combine these influences in finding the optimal AV-delay the mean effective AV-delay is introduced, which is a parameter that takes into account both right and left sided effective AV-delay. The new parameter corrects for variances in atrial and ventricular activation and for effective AV-delay in the right and left heart. The use of the mean effective AV-delay may improve automated algorithms for AV-delay optimization.

Chapter $\mathbf{5}$ focuses on the SonR1 signal, which is an accelerometer derived parameter reflecting the first heart sound, used for automated AV- and VV-delay optimization. The objective is to better understand the role of ventricular contractility, AV-coupling and IVD on the amplitude of SonR1 using canines with synchronous and dyssynchronous hearts. First, we show that during infusion of increasing concentrations of dobutamine the amplitude of SonR1 strongly correlates to RV and LV dP/dt $t_{\max }$. Next, the SonR signal was compared to various hemodynamic parameters during a large range of AV-delays. Starting from long AV-delays, SonR1 amplitude increases at intermediate AV-delays and is largest during the shortest AV-delays, possibly caused by atrial contraction against closed AV-valves. The optimal AV-delay, defined as the AV-delay with the largest increase in LV dP/dt max occurs at AV-delays just longer than those where the SonR1 amplitude starts to increase. Finally, the SonR1 values do not greatly vary between BiV and LV pacing, which indicates that IVD is not a major determinant of SonR1. The SonR1 amplitude is larger when measured in the $\mathrm{RV}$ than in the RA, but the relative increases at the shortest AV-delays were larger in the RA. We conclude that the SonR1 amplitude is influenced by changes in cardiac contractility 
and time interval between atrial and ventricular contraction, while IVD plays a minor role. Placement of the SonR sensor in the RA seems to assess overall cardiac function better than an RV-placement.

In chapter 6 we used a canine AV-block model and CircAdapt computer simulations to study the effect of a large variation of $A V$ - and $V V$-delays on RV and LV contractility. In the dogs we show that $R V$ pre-excitation results in the largest $L V$ total activation time and viceversa, while during simultaneous pacing two electrical wavefronts fuse and resynchronize the heart, indicated by the lowest LV total activation time. Additionally, RV function is best during RV pre-excitation, while LV pre-excitation results in the highest LV dP/dt $t_{\max }$ and decreases $\mathrm{RV} \mathrm{dP} / \mathrm{dt}_{\max }$. In contrast to changes in $\mathrm{VV}$-delay, alterations in $\mathrm{AV}$-delay have less effect on measured $\mathrm{LV}$ and $\mathrm{RV} \mathrm{dP} / \mathrm{dt}_{\max }$. These observations were replicated using a dogspecific version of the the CircAdapt model. We conclude that the LV and the RV respond in a opposite manner to variation in the amount of LV or RV pre-excitation. Computer simulations capture LV and RV behavior during pacing delay variation and may be used in the design of new CRT optimization studies.

In order to find an explanation for the different behavior of cardiac output and RV and LV dP/ $\mathrm{dt}_{\text {max }}$ the time course of these parameters during the first beats after the start of a certain setting were compared. We find that stroke volume of both ventricles stabilizes after three beats and are the same for the RV and LV. However, the differences between LV and RV $\mathrm{dP} / \mathrm{dt}_{\text {max }}$ remain present, accompanied by opposite changes in RV and LV end-diastolic volume. This study demonstrates that improving LV function can reduce RV function. Furthermore, cardiac output is not necessarily increasing when LV dP/dt ${ }_{\max }$ increases. Hence, an exclusive focus on the LV might not lead to the best overall outcome.

Finally, chapter $\mathbf{7}$ integrates the major findings of the above summarized chapters and discusses them in a broader scientific and clinical perspective. Based on this thesis we can conclude that restoring AV-coupling using BiV pacing results in acute hemodynamic improvements in both pigs and patients with heart failure and first-degree AV-block. The mechanism of action is due to better ventricular filling because of increased transmitral forward flow and a decrease of diastolic mitral regurgitation. This may lead to a novel indication for pacemaker therapy in patients with prolonged PR-interval, although additional research needs to further unravel which pacing modality is most suitable and which patient category has the highest benefits.

Furthermore, the fact that restoring AV-coupling in first-degree AV-block improves hemodynamics, once again suggests that AV-delay optimization remains an important factor in improving the efficacy of pacemaker therapies like CRT. We describe that the optimal AV-delay is influenced by changes in interatrial delay and ventricular dyssynchrony and that AV-delay optimization should therefore not only focus on the left side of the heart, but also consider the right side. Novel parameters like the mean effective AV-delay and 
mechanical information like the SonR1 signal, have the potential to play an important role in optimization strategies. Ultimately, ambulatory optimization of programmed AV-delays should focus on left and right sided AV-delays and make use of a combination of electrical and mechanical parameters. 


\section{SAMENVATTING}

Pacemakertherapie wordt gebruikt om het normale hartritme van patiënten in stand te houden en om de pompfunctie van het hart te verbeteren. Dit proefschrift richt zich vooral op dat laatste. De meest bekende toepassing is cardiale resynchronisatie therapie (CRT) ter behandeling van geleidingsstoornissen van het hart zoals linkerbundeltakblok (LBTB). Bij een LBTB knijpt eerst de rechterkamer en daarna pas de linkerkamer terwijl de kamers normaal synchroon knijpen. Hierdoor wordt de pompfunctie van het hart minder effıciënt. LBTB wordt op het hartfilmpje gekarakteriseerd door een verlengde QRS-duur (>130ms). CRT zorgt er met een pacemaker voor dat de kamers weer tegelijkertijd samenknijpen, waardoor de knijpkracht beter wordt en er meer bloed rond gepompt kan worden. Er is echter nog maar weinig bekend over de mogelijkheid om ook patiënten met een eerstegraads AV-blok te behandelen met pacemaker therapie. Bij een eerstegraads AVblok is er een vertraging (>200ms) tussen de activatie van de boezems (atria) en kamers (ventrikels), waardoor de vulling van het hart verminderd wordt en er een terugstroom kan optreden van bloed over de kleppen tussen de boezems en de kamers. Dit heeft als gevolg dat het hart minder bloed kan uitpompen.

Het onderzoek dat in dit proefschrift beschreven wordt heeft twee hoofddoelstellingen. Het eerste doel is om te onderzoeken of pacemakertherapie de functie van het hart van patiënten met een eerstegraads AV-blok kan verbeteren. Het tweede doel is om te verkennen hoe we de optimalisatie van de pacemaker instellingen kunnen verbeteren. Hierbij wordt onder andere gekeken naar de ideale (optimale) tijd tussen de activatie van de boezems en de kamers. Daarbij wordt niet alleen gekeken naar de functie van de linkerkamer, maar ook naar de rechterkamer, omdat het uiteindelijk de functie van beide kamers is die de kwaliteit van de bloedsomloop bepalen.

In hoofdstuk 2 beginnen we met een overzicht van de literatuur over de elektrofysiologische en de hemodynamische veranderingen (de eigenschappen van de bloedstroom in het hart- en vaatstelsel) die optreden tijdens CRT bij zowel dieren als patiënten. Zoals gezegd, leidt LBTB tot een asynchrone elektrische activatie van de linker- en rechterkamer (dyssynchronie tussen de kamers) en veroorzaakt daardoor een inefficiënte samentrekking met een verlaagd hartminuutvolume (de hoeveelheid bloed die het hart per minuut pompt). Door de kamers tegelijkertijd te pacen wordt deze elektrische dyssynchronie vermindert wat resulteert in een acute verbetering van het slagvolume (de hoeveelheid bloed die wordt uitgepompt) en toegenomen knijpkracht van de linkerkamer ( $\mathrm{LV} \mathrm{dP} / \mathrm{dt}_{\text {max }}$ ). Tot slot wordt het belang van het instellen van de optimale vertraging tussen de boezems en kamers (AV-interval) en tussen de kamers onderling ( $\mathrm{V}$-interval) besproken.

Het doel van hoofdstuk $\mathbf{3}$ is om te onderzoeken welk effect het herstellen van het interval tussen de activatie van de boezems en kamers bij een eerstegraads AV-blok heeft. Dit onderzoek is een combinatie van dierproeven, computermodellen en een patiëntenstudie. 
In het dierexperiment laten we zien dat het optimaliseren van het AV-interval door beide kamers tegelijkertijd te pacen (biventriculair) resulteert in een toename van bloeddruk en hartminuutvolume in vergelijking met de situatie tijdens een experimenteel eerstegraads AV-blok. Vergelijkbare resultaten worden gevonden bij patiënten met hartfalen en een eerstegraads AV-blok maar zonder LBTB. Met behulp van inwendig gemeten druk-volume diagrammen vinden we dat de verbetering in de pompfunctie van het hart wordt veroorzaakt door een betere vulling van de linkerkamer. Dit is het resultaat van een combinatie van een toegenomen voorwaartse stroom over de mitralisklep (klep tussen linkerboezem en linkerkamer) en een afname in het terug lekken van bloed van kamer naar boezem over deze klep aan het einde van de ontspanningsfase van het hart (diastole). Deze resultaten werden ook gerepliceerd met behulp van het CircAdapt-computermodel, wat aanduidt dat de verbetering in pompfunctie door de pacemakertherapie verklaard kan worden op grond van de fysische principes die als aannames dienden in het computermodel.

In de patiëntenstudie laten we zien dat het optimaliseren van het AV-interval met biventriculair pacen het slagvolume verhoogt, maar dat deze verbetering afwezig is bij het gebruik van rechterkamer pacen. Dit nadelige effect van rechterkamer pacen wordt veroorzaakt door een toename van dyssynchronie tussen de kamers en kan ook worden gesimuleerd in CircAdapt. Deze resultaten geven aan dat het optimaliseren van het AVinterval aanzienlijke voordelen kan opleveren voor patiënten door verbetering van het slagvolume, zolang een toename van dyssynchronie tussen de kamers wordt vermeden.

In hoofdstuk $\mathbf{4}$ proberen we beter inzicht te krijgen hoe het hart zich vult als het gepaced wordt met verschillende AV-intervallen. Verder onderzoeken we hoe variaties in de vertraging tussen de rechterboezem en linkerboezem en verschillende activaties van de kamers (met verschillende mate van dyssynchronie tussen de kamers) het optimale AVinterval beïnvloeden. De vertraging tussen beide boezems is afhankelijk van het feit of de rechterboezem gepaced wordt of niet. De activatie van de kamers hangt af van de plaats(en) waar deze worden gepaced. Daarnaast is het van belang niet alleen naar de functie van de linkerkamer te kijken, maar ook naar de functie van de rechterkamer.

De experimenten werden uitgevoerd in varkens waarbij verschillende vertragingen tussen de boezems en kamers met elkaar werden vergeleken. Er werd onderscheid gemaakt tussen het pacen van de boezem of het volgen van de eigen intrinsieke hartfrequentie. Dit laatste wordt sensen genoemd en hierbij wordt de boezem dus niet gepaced. De kamers werden vervolgens op verschillende manieren geactiveerd; rechterkamer pacen, linkerkamer pacen of biventriculair pacen. Onze bevindingen zijn dat het AV-interval met het hoogste hartminuutvolume (het optimale AV-interval) afhankelijk is van of er in de boezem gepaced of gesensed wordt en van de locatie waar de kamers gepaced worden. Het optimale AVinterval is langer als de rechterboezem gepaced wordt, omdat de linkerboezem dan later geactiveerd wordt. Ook als de linkerkamer eerder dan de rechterkamer wordt geactiveerd is het optimale AV-delay langer. In beide gevallen is het interval tussen linkerboezem 
en linkerkamer korter dan het interval tussen de rechterboezem en rechterkamer. Het gemiddelde effectieve AV-delay is een nieuwe parameter die rekening houdt met de vertraging tussen boezem en kamer van beide harthelften. Deze waarde corrigeert voor afwijkingen in activatie van de boezems en de kamers. In de toekomst kan dit mogelijk gebruikt worden om het AV-interval in pacemakers automatisch te optimaliseren.

Hoofdstuk 5 richt zich op het gebruik van een versnellingsmeter (accelerometer) in een pacemakerdraad die gebruikt kan worden voor de optimalisatie van de vertraging tussen de boezems en de kamers en tussen de kamers onderling. De accelerometer registreert trillingen en vibraties in het hart en komt overeen met de eerste harttoon. Het doel van ons onderzoek was om het zogenaamde SonR1 signaal beter te leren begrijpen en te bestuderen welke invloed de knijpkracht van het hart, het AV-interval en de dyssynchronie tussen de kamers hierop heeft.

Hiervoor werden experimenten gedaan in honden met harten die synchroon (smal QRScomplex) en dyssynchroon (LBTB) geactiveerd werd. Allereerst laten we zien dat de grootte (amplitude) van het SonR1 signaal correleert met de knijpkracht van zowel de linkerals rechterkamer, door de knijpkracht medicamenteus te versterken met dobutamine. Vervolgens testen we verschillende hemodynamische waarden tijdens het pacen van de rechterboezem en kamers met verschillende AV-intervallen. Hierbij gebruiken we signalen uit zowel de rechterboezem als rechterkamer. De amplitude van het SonR1 signaal is het laagst als de vertraging tussen de boezem en kamers activatie het langst is. De amplitude wordt groter naarmate het AV-interval korter wordt en stijgt exponentieel tijdens extreem korte AV-intervallen. Dit laatste kan verklaard worden doordat bij deze korte AV-intervallen de boezems samentrekken terwijl ook de kamers al geactiveerd zijn waardoor de kleppen tussen de boezems en kamers zich al sluiten, resulterend in verhoogde trillingen en vibraties in het hart. Het verschil in amplitude verschilt niet tussen linkerkamer pacen en biventriculair pacen. We concluderen dat de SonR1-amplitude wordt beïnvloed door veranderingen in knijpkracht en het tijdsinterval tussen activatie van de boezem en kamers, terwijl de mate van dyssynchronie een ondergeschikte rol lijkt te spelen. Plaatsing van de SonR-sensor in de rechterboezem lijkt de algehele hartfunctie beter te beoordelen dan een sensor geplaatst in de rechterkamer.

In hoofdstuk 6 gebruiken we honden met een totaal AV-blok en CircAdapt computersimulaties om de invloed van pacen met verschillende vertragingen tussen de boezems en de kamers en tussen de kamers onderling te bestuderen. We onderzoeken wat de invloed hiervan is op de kracht waarmee de linker- en rechterkamer samentrekken. Vervolgens testen we hoe deze verschillen in knijpkracht leiden tot veranderingen in het hartminuutvolume. In honden zien we dat de knijpkracht van de linker- of rechterkamer toeneemt als deze eerder dan de andere wordt geactiveerd (pre-excitatie). Het veranderen van de vertraging tussen de boezems en de kamers lijkt dan weer weinig invloed te hebben op de knijpkracht van het hart. Deze waarnemingen werden bevestigd met het CircAdapt 
computer model, waarin meetgegevens uit de dierproeven werden gebruikt om een "hondspecifieke" versie van dit model te maken. We concluderen dat de linker- of rechterkamer in tegenovergestelde richting reageren op pre-excitatie. Dit betekent dat de pacemaker setting die het hoogste hartminuutvolume oplevert ongeveer een gemiddelde is van de instellingen die de hoogste rechter- en linkerkamer knijpkracht opleveren.

Tot slot integreert hoofdstuk $\mathbf{7}$ de belangrijkste bevindingen van de hierboven samengevatte hoofdstukken. Op basis van dit proefschrift kunnen we concluderen dat het optimaliseren van de vertraging tussen de boezems en de kamers met behulp van biventriculair pacen resulteert in een verbetering van het hartminuutvolume en een hogere bloeddruk bij zowel varkens als patiënten met hartfalen en eerstegraads AV-blok. De belangrijkste reden hiervoor is een betere vulling van de linkerkamer als gevolg van toegenomen voorwaartse stroming over de mitralisklep in combinatie met een afname van de terugstroom van bloed naar de linkerboezem. Dit leidt mogelijk tot een nieuwe indicatie voor pacemakertherapie bij patiënten met een eerstegraads AV-blok, hoewel aanvullend onderzoek verder moet ontrafelen welke manier van pacen het meest geschikt is en welke patiënten exact in aanmerking komen.

Het feit dat het herstellen van de geleiding tussen de boezems en kamers bij een eerstegraads AV-blok de hemodynamiek verbetert, suggereert dat het optimaliseren van het AV-interval een belangrijke factor blijft bij het verbeteren van de effectiviteit van pacemakers. We laten zien dat de optimale vertraging tussen de boezems en kamers afhankelijk is van de activatie van de boezems en van de plek waar de kamers gepaced worden. We moeten hierbij niet alleen maar focussen op de linkerkamer, maar juist ook rekening houden met de rechterharthelft. Nieuwe parameters zoals het gemiddelde effectieve AV-interval en mechanische informatie zoals het SonR1-signaal kunnen een belangrijke rol spelen in optimalisatiestrategieën. Uiteindelijk zou automatische optimalisatie van het AV-interval plaats moeten vinden op basis van een combinatie van elektrische en mechanische parameters. 



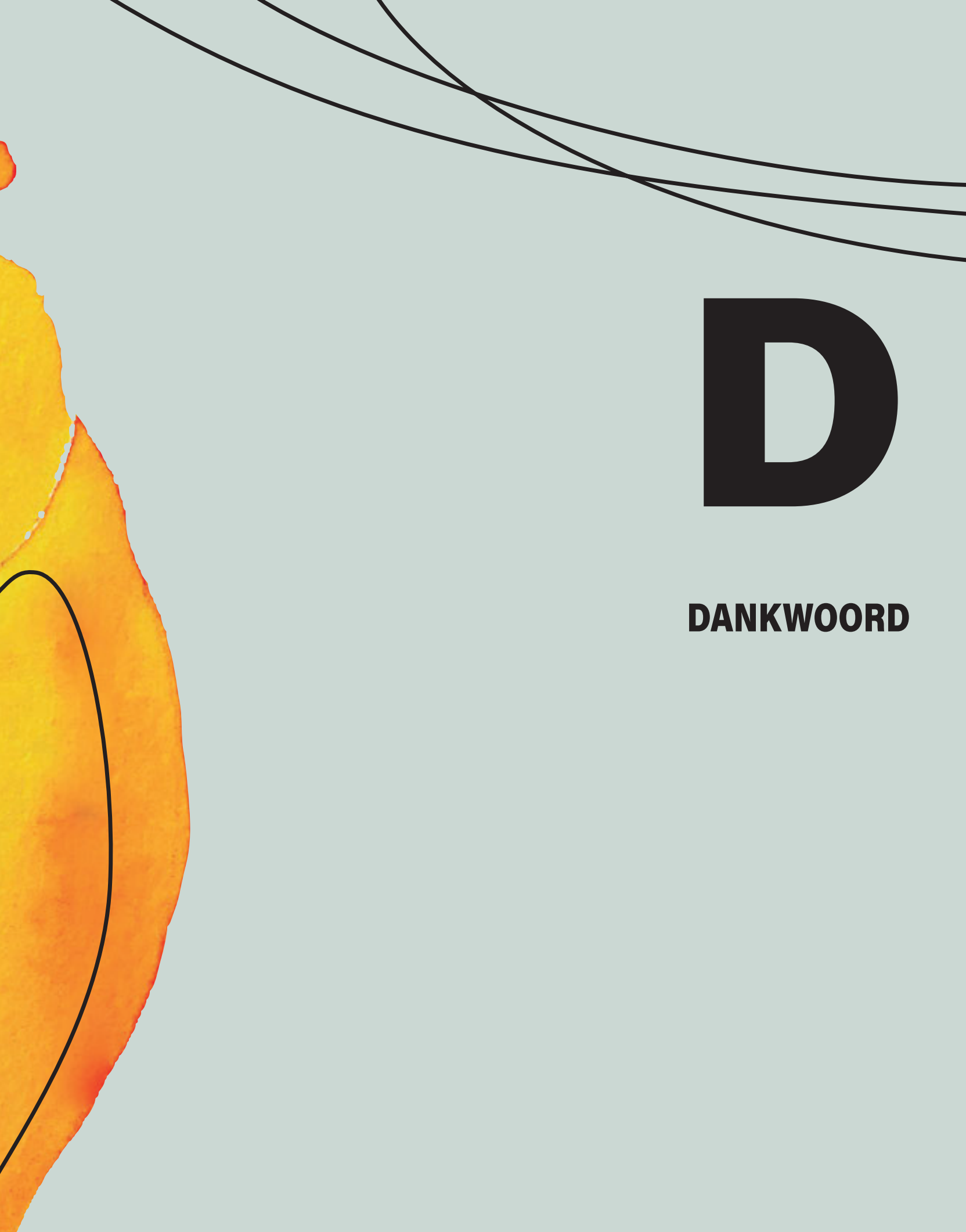

DANKWOORD 



\section{DANKWOORD}

Een proefschrift is natuurlijk niet compleet zonder dankwoord. Veel mensen hebben direct of indirect hun bijdrage geleverd aan het tot stand komen van dit boekwerk. Mensen die mij goed kennen weten dat ik het niet makkelijk vind om mijn emotionele kant te laten zien, maar hierbij doe ik toch een bescheiden poging.

Allereerst wil ik natuurlijk mijn promotoren bedanken. Zonder jullie zou dit proefschrift nooit tot stand zijn gekomen. Professor Prinzen, allerbeste Frits, onze wetenschappelijke paden kruisten al heel lang geleden en het is daarom niet verrassend dat ik onder jouw bezielende begeleiding promoveer. Ik wil je danken voor het feit dat je altijd in mij geloofd hebt ondanks mijn soms toch wat nonchalante houding. Zonder jouw snelle, directe en uitgebreide feedback zou ik nu nog steeds bezig zijn met het schrijven van het manuscript. Misschien had ik ook naar je moeten luisteren en mijn fiets-carrière op moeten geven om te gaan hardlopen. Gelukkig maakte je er nooit een probleem van dat ik tijdens werktijd de Tour de France zat te kijken... Bedankt voor alles!

Professor Maessen, beste Jos, als net afgestudeerde basisarts kreeg ik de gelegenheid om bij de CTC mijn eerste stappen binnen de medische wereld te zetten. Ik wil je bedanken voor de mogelijkheid om een full-time PhD-traject te combineren met klinische werkzaamheden binnen de hartchirurgie, ook al was Frits daar niet altijd even blij mee. Uiteraard ben ik je ook zeer dankbaar voor het feit dat ik me nu cardiothoracaal chirurg in opleiding mag noemen. Het is goed om te weten dat je deur altijd open staat voor een luisterend oor.

De voorzitter en leden van de beoordelingscommissie, professor Schotten, professor Brunner-La Rocca, professor Lorusso, professor Bogers en professor Timmermans, wil ik danken voor hun kritische maar positieve beoordeling van dit proefschrift.

Ik ben blij om deel uitgemaakt te hebben van de CRT-groep; een multidisciplinaire groep wetenschappers bestaande uit enthousiaste fysiologen, cardiologen en biomedische techneuten. Marion bedankt voor alles wat je me geleerd hebt op de dieren OK; zonder jou zouden de dierproeven nooit tot een goed einde gebracht zijn. Floor en Nienke, dank voor het draagbaar maken van de eindeloze pace protocollen. Peter en Erik, thanks voor alle computersimulaties die jullie gedraaid hebben met CircAdapt. Verder wil ik Kevin Vernooy en Joost Lumens bedanken voor hun betrokkenheid, relevante input en kritische noot. Ook alle andere deelnemers aan de werkbesprekingen, bedankt voor het meedenken en de fijne samenwerking!

Part of the animal experiments were conducted at the Institute Mutualiste Montsouris in Paris. Filippo, Fabrizio, Danny, Amel and Nicolas, thanks a lot for the opportunity and the lovely time we had in Paris. Grazie mille a tutti! 
Uiteraard wil ik alle leden van de staf cardiothoracale chirurgie van het MUMC+ bedanken voor het in mij gestelde vertrouwen. Het is een genot om onder jullie supervisie te mogen groeien tot volwaardig hartchirurg en ik ben ervan overtuigd dat ik in de toekomst nog regelmatig terug zal denken aan jullie wijze lessen. Een speciaal woord van dank dien ik te richten aan mijn opleider Suzanne Kats; het is ontzettend fijn om een opleider te hebben die niet alleen naar de professionele maar juist ook naar de privé kant van de AIOS kijkt! Ook dank aan Meindert Sosef en Erik de Loos, opleiders algemene heelkunde in het Zuyderland MC, voor de fijne en leerzame tijd aldaar.

In mijn jaren bij de CTC heb ik veel arts-assistenten zien komen en gaan. ledereen bedankt voor de prettige en gezellige samenwerking. Speciale dank aan Lars, Sam, Pieter en Martijn voor alle memorabele momenten die we vooral ook buiten de kliniek beleefd hebben. Mooi om te zien dat iedereen goed op zijn plek is gekomen. Alle PA'ers en verpleegkundige specialisten, met name Sandra, Palmyre en Martijne, bedankt voor het vormen van de ruggengraat van de CTC. Laat ik tenslotte de dames van het planbureau en het secretariaat niet vergeten te bedanken voor de smalltalk en al het essentiële werk dat jullie achter de schermen verrichten.

Hartchirurgie is een teamsport en zou dan ook niet mogelijk zijn zonder de inzet van alle cardiologen, cardio-anesthesisten en intensivisten. ledereen bedankt voor de bijdrage en de prettige samenwerking. Daarnaast moeten alle andere collega's op de OK en de verpleegafdelingen niet vergeten worden. Mede dankzij jullie inzet maar ook flauwekul en gezelligheid, kom ik iedere dag weer met plezier naar mijn werk.

Ik vind mijn werk ontzettend tof, maar ik leef niet om te werken. Er zijn een hoop mensen buiten mijn werk die een plaats verdienen in het dankwoord van dit proefschrift. Niet zo zeer omdat ze een bijdrage hebben geleverd aan het tot stand komen hiervan, maar wel om hun continue interesse in de voortgang van mijn promotie. En misschien nog wel meer omdat ze zorgen voor de broodnodige ontspanning die nodig is om hard werken mogelijk te maken.

Allereerst René, Guus en Tim. Vrienden van het eerste uur, vrienden van de middelbare school. Samen hebben we ontzettend veel gave dingen meegemaakt en er zullen vast nog veel mooie momenten volgen. René, in de buurt van (vrouwelijke) slachtoffers van onze slechte grappen, is één blik vaak genoeg om te weten wat de ander van plan is. Guus, na ontelbare stoeipartijen weten we nog steeds niet wie de sterkste is. Gelukkig zijn we nu volwassen, trotste papa's en stoeien we alleen nog met onze kleine mannen. En Tim, ik blijf graag langskomen in Kessel ondanks het feit dat je toch wel echt harder fietst dan ik. Wanneer gaat er weer een varken aan het spit? Tegenwoordig wonen we helaas allemaal erg verspreid over het land en zien we elkaar te weinig, maar gelukkig doet dit geen afbreuk aan onze vriendschap. Het is fijn om te weten dat ik altijd op jullie kan terugvallen. Volgens 
mij is het tijd dat we weer eens alderwets Venlo, het stedje van lol en plezeer, op zijn kop gaan zetten!

Door de hele corona-crisis zie ik de Annorlunda mannen helaas minder dan voorheen, maar de momenten dat we samen zijn blijven momenten van ultieme ontspanning. Vaak vergezeld van een biertje en altijd van een hoop humor en plezier. Joey, mijn oude huisgenoot, je blijft een prachtige vent, we moeten vaker fietsen! En over fietsen gesproken, zonder de Brullende Condors zou dit proefschrift waarschijnlijk al enkele jaren eerder afgerond zijn! Rein, volgend jaar heb ik weer meer tijd om je uit te dagen op de Limburgse heuvels, of ben je dan zelf te druk met je promotie?

Een tweetal mannen die hierboven al aan bod zijn gekomen wil ik nog eens extra benoemen. René, we kennen elkaar al meer dan de helft van ons leven waardoor ik met niemand zo'n goede wisselwerking heb als met jou. Ondanks verwoede pogingen om het einde van je studententijd maar te blijven uitstellen, heb je het tegenwoordig toch maar goed voor mekaar. Het feit dat jij als niet-wetenschapper je promotie zo snel afgerond hebt maakt me trots (en een beetje jaloers). Als jouw getuige en paranimf heb ik je uitgebreid in het zonnetje mogen zetten, en ik kan niet wachten op het moment dat jij dit bij mij mag doen! Dikke kus voor Eva.

Sammie, hoogbegaafde ras idioot. Ik ken niemand die zo intelligent is als jij. Ik blijf onder de indruk van jouw wetenschappelijke kwaliteiten en nu blijk je ook nog getalenteerd te zijn op de OK. Gelukkig gaat dit allemaal niet ten koste van je sociale kant en kan ik blijven genieten van je alter-ego Seumpie Haets. Het is een genot om met je samen te werken en je bent veel meer dan een goede collega, getuige het feit dat je me vaker belt dan dat Bente doet. Is Isis niet jaloers? Met jou als paranimf naast me wordt die verdediging een eitje!

Ze zeggen wel eens dat je je schoonfamilie niet voor het uitzoeken hebt. Wat dat betreft heb ik geluk, want mijn schoonfamilie voelt als een warm bad. Kos en Henny, Ineke en Wout, ik bof maar met jullie als schoonouders. Een tweede huis in Frankrijk, een gloednieuw zwembad in de tuin; je zou het slechter kunnen treffen. Buiten dat, en natuurlijk veel belangrijker, bedankt voor alle gezelligheid en liefde die we van jullie ontvangen. Luuk, wanneer plannen we weer een beachparty op Sicilië? En Rosie, super bedankt voor de prachtige schildering die mijn kaft siert. Samia en Mak, het zou tof zijn als onze kleine boefjes zulke goede vriendjes blijven!

Langzaam komen we bij de personen die me het meest dierbaar zijn. Heel belangrijk zijn mijn broertje en zusjes. Het was fantastisch om op te groeien in een gezin met vier kinderen, ook al wisten veel mensen niet dat jullie nog een oudere broer hadden omdat ik zo vroeg het huis uit was. Ik ben super trots op jullie alle drie! Debbie, ik ben onder indruk van de ontwikkeling die jij hebt doorgemaakt en kan oprecht genieten van het gezinnetje dat jij en Dave hebben gesticht; Mace en Kess zijn schatjes! Kim, misschien lijken wij wel 
het meest op elkaar. Ik weet zeker dat jij een fantastische carrière tegemoet gaat met een prachtige toekomst voor jou en Sjors in het verschiet. Tenslotte Ray, kleine broertjes worden groot (en breed). Superknap hoe jij je met je eigen sportschool door de corona-crisis hebt geslagen en ik ben ervan overtuigd dat er voor jou en Sharon nog veel meer moois aan zit te komen. Thanks for being who you are!

Uiteraard een speciaal woord van dank aan mijn ouders. Jullie hebben ervoor gezorgd dat ik al mijn dromen waar heb kunnen maken. Dankzij jullie ben ik nooit iets te kort gekomen en door jullie stimulans ben ik de persoon die ik nu ben. Jullie hebben tot vervelens toe geïnformeerd hoe het nou eigenlijk met mijn promotie zat, maar dat heeft me alleen maar gemotiveerd om door te zetten. Mam, het is mooi om te zien dat ik met onze gedeelde interesse voor het hart jouw dromen nu ook waarmaak. Pap, fijn dat je je geluk hebt gevonden bij Lizet en je blijft een rolmodel voor mij als vader voor mijn eigen kinderen. Bedankt dat jullie er altijd voor ons zijn!

En als het over ons gaat dan bedoel ik natuurlijk Bente en onze zoontjes. Allerliefste Bente, jou ben ik het meest dankbaar van iedereen. Niet alleen voor je geduld en je steun tijdens het tot stand komen van dit proefschrift, maar vooral ook voor alles wat je daarnaast voor me doet. Het is een genot om jou in mijn leven te hebben. Bedankt voor alle lol en je onvoorwaardelijke liefde, maar ook voor je eerlijkheid en de spiegel die je me af en toe voorhoudt. Sorry dat ik af en toe een autistische en eigenwijze pain in the ass ben... Ik ben echt enorm trots op jou als persoon en op wat je allemaal al bereikt hebt in je eigen carrière. Het lijkt je zo makkelijk af te gaan. Buiten dat ben je ook nog eens de allerbeste mama voor Tijmen en Julian. Sinds deze twee boefjes in ons leven zijn heeft liefde een andere dimensie gekregen. De rest is ineens een stuk minder belangrijk. Ik kijk er naar uit om de tijd die vrij komt na het afronden van dit 'boekje' met jullie te mogen besteden! Love you long time! 





\section{CURRICULUM VITAE}

Rick Schreurs, full name Henricus Hendrikus Hermanus Schreurs, was born on 14 December 1985 in Venlo, the Netherlands. After graduating from College Den Hulster (TVWO) in 2004, he went to study General Health Sciences at Maastricht University. In 2007 he received his Bachelor of Science in Movement Sciences and Bioregulation \& Health. After that he started a master in Cardiovascular Biology and Medicine (CARIM master) where he followed an internship at the department of Physiology of Maastricht University. This was where he first met professor F.W. Prinzen, and it was under his supervision he completed his thesis to receive the Master of Philosophy title. Next, he finally was able to start his medical training when he was accepted to the Medicine and Clinical Research master (A-KO) at the Faculty of Health, Medicine and Life Sciences of Maastricht University. In the last year of this master, he once again participated in a traineeship at the department of Physiology and he completed a 10-week internship at the department of Cardiothoracic Surgery of the Maastricht University Medical Centre+. After completing his medical master, he started his clinical career at the department of Cardiothoracic Surgery as a resident not in training. In 2015 he started his PhD project at the department of Physiology under supervision of professor F.W. Prinzen and professor J.G. Maessen. Then in 2018 he was lucky to start his residency in Cardiothoracic Surgery at the Maastricht University Medical Centre+ under supervision of Dr. S. Kats and professor J.G. Maessen. In 2019 he spent one year at the General Surgery department of Zuyderland Medical Centre under supervision of Dr. M. Sosef and Drs. E. de Loos before returning to Maastricht in 2020. Finally, in 2021 he was able to complete his PhD project with this thesis as ultimate result. 


\section{LIST OF PUBLICATIONS}

Schreurs R, Wiegerinck RF, Prinzen FW. Exploring the Electrophysiologic and Hemodynamic Effects of Cardiac Resynchronization Therapy: From Bench to Bedside and Vice Versa. Card Electrophysiol Clin. 2015;7(4):599-608.

Wiegerinck RF, Schreurs R, Prinzen FW. Pathophysiology of dyssynchrony: of squirrels and broken bones. Neth Heart J. 2015.

Meani P, Gelsomino S, Natour E, Johnson DM, Rocca HB, Pappalardo F, Bidar E, Makhoul M, Raffa G, Heuts S, Lozekoot P, Kats S, Sluijpers N, Schreurs R, Delnoij T, Montalti A, Sels JW, van de Poll M, Roekaerts P, Poels T, Korver E, Babar Z, Maessen J, Lorusso R. Modalities and Effects of Left Ventricle Unloading on Extracorporeal Life support: a Review of the Current Literature. Eur J Heart Fail. 2017;19 Suppl 2:84-91.

Schreurs R, Heuts S, Natour E, Lorusso R. The unstoppable heart: an aortocoronary fistula in coronary artery bypass grafting. Interact Cardiovasc Thorac Surg. 2018;27(6):912-3.

Meani P, Delnoij T, Raffa GM, Morici N, Viola G, Sacco A, Oliva F, Heuts S, Sels JW, Driessen R, Roekaerts P, Gilbers M, Bidar E, Schreurs R, Natour E, Veenstra L, Kats S, Maessen J, Lorusso R. Protracted aortic valve closure during peripheral veno-arterial extracorporeal life support: is intra-aortic balloon pump an effective solution? Perfusion. 2019;34(1):35-41.

Meani P, Matteucci M, Jiritano F, Fina D, Panzeri F, Raffa GM, Kowalewski M, Morici N, Viola G, Sacco A, Oliva F, Alyousif A, Heuts S, Gilbers M, Schreurs R, Maessen J, Lorusso R. Long-term survival and major outcomes in post-cardiotomy extracorporeal membrane oxygenation for adult patients in cardiogenic shock. Ann Cardiothorac Surg. 2019;8(1):11622.

Willemen E, Schreurs R, Huntjens PR, Strik M, Plank G, Vigmond E, Walmsley J, Vernooy K, Delhaas T, Prinzen FW, Lumens J. The Left and Right Ventricles Respond Differently to Variation of Pacing Delays in Cardiac Resynchronization Therapy: A Combined Experimental- Computational Approach. Front Physiol. 2019;10:17.

Kowalewski M, Raffa G, Zielinski K, Meani P, Alanazi M, Gilbers M, Heuts S, Natour E, Bidar E, Schreurs R, Delnoij T, Driessen R, Sels JW, van de Poll M, Roekaerts P, Maessen J, Suwalski P, Lorusso R. Baseline surgical status and short-term mortality after extracorporeal membrane oxygenation for post-cardiotomy shock: a meta-analysis. Perfusion. 2020;35(3):246-54. 
Kowalewski M, Raffa GM, Zielinski K, Alanazi M, Gilbers M, Heuts S, Natour E, Bidar E, Schreurs R, Delnoij T, Driessen R, Sels JW, van de Poll M, Roekaerts P, Meani P, Maessen J, Suwalski P, Lorusso R. The impact of Centre's heart transplant status and volume on in-hospital outcomes following extracorporeal membrane oxygenation for refractory postcardiotomy cardiogenic shock: a meta-analysis. BMC Cardiovasc Disord. 2020;20(1):10.

Kowalewski M, Zielinski K, Gozdek M, Raffa GM, Pilato M, Alanazi M, Gilbers M, Heuts S, Natour E, Bidar E, Schreurs R, Delnoij T, Driessen R, Sels JW, van de Poll M, Roekaerts P, Pasierski M, Meani P, Maessen J, Suwalski P, Lorusso R. Veno-Arterial Extracorporeal Life Support in Heart Transplant and Ventricle Assist Device Centres. Meta-analysis. ESC Heart Fail. 2021;8(2):1064-75

Marcuse F, Rutten M, Schreurs R, Gietema HA, Theunissen P, Maessen JG. Symptomatic mediastinal mass in a 32-year-old male. Breathe. 2021;17:210029 

<smiles>C1=CC=C1</smiles> 